\title{
Energy Pathways and Structures of Oceanic Eddies from the ECCO2 State Estimate and Simplified Models
}

\author{
by \\ $\mathrm{Ru}$ Chen
}

B.S., Ocean University of China, 2006

Submitted in partial fulfillment of the requirements for the degree of Doctor of Philosophy

at the

MASSACHUSETTS INSTITUTE OF TECHNOLOGY

and the

\section{WOODS HOLE OCEANOGRAPHIC INSTITUTION}

February 2013

(C) $2013 \mathrm{Ru}$ Chen. All rights reserved.

The author hereby grants to MIT and WHOI permission to reproduce and to distribute publicly paper and electronic copies of this thesis document in whole or in part.

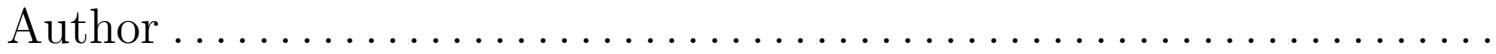

Joint Program in Physical Oceanography - Massachusetts Institute of Technology / Woods Hole Oceanographic Institution January 4, 2013

Certified by .

Carl Wunsch

Cecil and Ida Green Professor of Physical Oceanography Thesis Supervisor

Certified by

Glenn Flierl

Professor of Oceanography Thesis Supervisor

Accepted by . .

Glenn Flierl

Chair, Joint Committee for Physical Oceanography 


\title{
Energy Pathways and Structures of Oceanic Eddies from the ECCO2 State Estimate and Simplified Models
}

by

\author{
$\mathrm{Ru}$ Chen \\ Submitted to the Joint Program in Physical Oceanography - Massachusetts \\ Institute of Technology / Woods Hole Oceanographic Institution \\ on January 4, 2013, in partial fulfillment of the \\ requirements for the degree of \\ Doctor of Philosophy in Physical Oceanography
}

\begin{abstract}
Studying oceanic eddies is important for understanding and predicting ocean circulation and climate variability. The central focus of this dissertation is the energy exchange between eddies and mean flow and banded structures in the low-frequency component of the eddy field. A combination of a realistic eddy-permitting ocean state estimate and simplified theoretical models is used to address the following specific questions. (1) What are the major spatial characteristics of eddy-mean flow interaction from an energy perspective? Is eddy-mean flow interaction a local process in most ocean regions? (2) The banded structures in the low-frequency eddy field are termed striations. How much oceanic variability is associated with striations? How does the time-mean circulation, for example a subtropical gyre or constant mean flow, influence the origin and characteristics of striations? How much do striations contribute to the energy budget and tracer mixing?
\end{abstract}

Thesis Supervisor: Carl Wunsch

Title: Cecil and Ida Green Professor of Physical Oceanography

Thesis Supervisor: Glenn Flierl

Title: Professor of Oceanography 


\section{Acknowledgments}

My deepest gratitude goes to my advisors Glenn Flierl and Carl Wunsch for their patient guidance, constant encouragement and support. They gave me the freedom to explore interesting topics and were available whenever I needed advice. Discussions with them were always illuminating. Their care and support helped me get through some tough times. Their integrity, insights, and enthusiasm to science and education will always be an inspiration for me.

I offer my sincere thanks to my thesis committee: Rui Xin Huang, Michael Spall, Steven Lentz and Raffaele Ferrari. Their insightful suggestions and constructive criticisms greatly improved the science and presentation of the thesis. They also shared with me their own research methdologies. John Marshall chaired my defense and provided useful feedbacks.

Many thanks go to Dimitris Menemenlis, Hong Zhang, Chris Hill, Jean-Michel Campin, Gaël Forget and Patrick Heimbach for discussions about the ECCO2 state estimate and the MITgcm. I appreciate the mentorship provided by Qinyu Liu, Karl Helfrich and Larry Pratt. I am grateful to Rui Xin Huang and Qinyu Liu for introducing me to the MIT/WHOI Joint Program.

My friends in the MIT and JP community made my stay in graduate school a colorful experience. I thank my classmates Wilken, Julian, Nicholas and Sophie. Jinbo, Dan, Anliang and Jareth provided helpful suggestions on early drafts. I also benefited from conversations with Cimarron, Holly, Hristina, Jessica, and so on. I appreciate the encouragement from Chen and Enying during my thesis writing period.

Special thanks go to my parents for their understanding and unconditional love.

This research was supported by the National Aeronautics and Space Administration contracts NNX09AI87G and NNX08AR33G. 


\section{Contents}

1 Introduction $\quad 11$

1.1 Motivation .......................... 11

1.2 Research problems . . . . . . . . . . . . . . . . . . 15

1.3 Methodology and outline . . . . . . . . . . . . . . . . . . . . . 19

2 The description of the ECCO2 state estimate 21

2.1 Introduction . . . . . . . . . . . . . . . . . . . 21

2.2 Model configuration . . . . . . . . . . . . . . . . . . . 22

2.3 The dynamically consistent state estimation method . . . . . . . . . . 27

2.4 Model-data comparison . . . . . . . . . . . . . . . . . . . 34

2.5 Conclusions .......................... . . . 43

3 A description of eddy-mean flow interaction in the ECCO2 state $\begin{array}{ll}\text { estimate } & 45\end{array}$

3.1 Introduction . . . . . . . . . . . . . . . . . . . 45

3.2 Diagnostic framework . . . . . . . . . . . . . . . . . 49

3.3 ECCO2 state estimate evaluation . . . . . . . . . . . . . . 59

3.4 Results ......................... 65

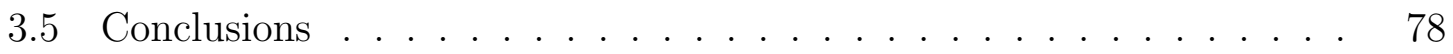




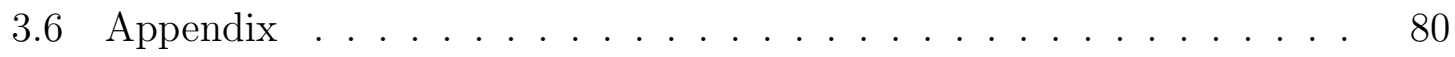

4 Quantify and interpret striations in a subtropical gyre from a spec$\begin{array}{ll}\text { tral perspective } & 89\end{array}$

4.1 Introduction . . . . . . . . . . . . . . . . . . . . 89

4.2 Basic description about striations in ECCO2 model . . . . . . . . . 92

4.3 Percentage of eddies associated with striations in ECCO2 model and

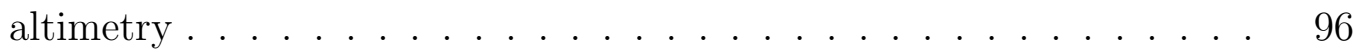

4.4 Effect of the gyre-flow on idealized striations . . . . . . . . . . . 104

4.5 Applications . . . . . . . . . . . . . . . . . . 127

4.6 Conclusions . . . . . . . . . . . . . . . . . . . . 133

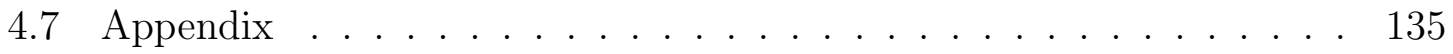

5 Striations and their contribution to the energy budget and tracer $\begin{array}{ll}\text { mixing in the barotropic system with mean flow } & 141\end{array}$

5.1 Introduction . . . . . . . . . . . . . . . . 141

5.2 Experiment setup . . . . . . . . . . . . . . . . . . . 143

5.3 Rhines jets vs. striations . . . . . . . . . . . . . . . . . . 144

5.4 Effect of mean flow on the energy pathway . . . . . . . . . . . 152

5.5 Effect of mean flow on total mixing and striations' contribution . . 158

5.6 Applications . . . . . . . . . . . . . . . . . . . . 163

5.7 Conclusions . . . . . . . . . . . . . . . . . . . . 165

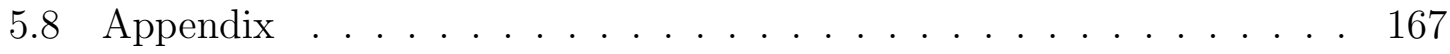

6 Conclusions 183

6.1 ECCO2 state estimate evaluation . . . . . . . . . . . . 183

6.2 Eddy-mean flow interaction in the global ocean . . . . . . . . . 184 
6.3 Striations in the time-mean circulation and their consequences . . . . 186 


\section{Chapter 1}

\section{Introduction}

\subsection{Motivation}

Oceanic variability occurs over all known spatial and temporal scales. This thesis focuses on the large-scale and mesoscale subinertial oceanic variability, which is hereafter termed eddies. Eddies are prevalent in the global ocean (Figure 1-1). Their dominant scales are 50 100 $\mathrm{km}$ in the off-equatorial region (Ferrari and Wunsch, 2009) and most eddy kinetic energy is located in the western boundary current regions and the Southern Ocean (Figure 1-2).

It is important to characterize oceanic eddies and explore eddy dynamics for the following reasons. First, eddies contain most kinetic energy in the world ocean, transport tracers (e.g. heat and salt), and they greatly influence the oceanic circulation through many processes. For example, the eddy field can influence water mass transformation by contributing to the total subduction and ventilation rates (e.g. Gebbie,

2007; Nishikawa et al., 2010; Lachkar et al., 2009). Also, eddies are key in balancing the heat budget in the overflow regions and the deep convection sites (e.g. Marshall and Schott, 1999; Voet and Quadfasel, 2010; Spall, 2012); thus they influence the 


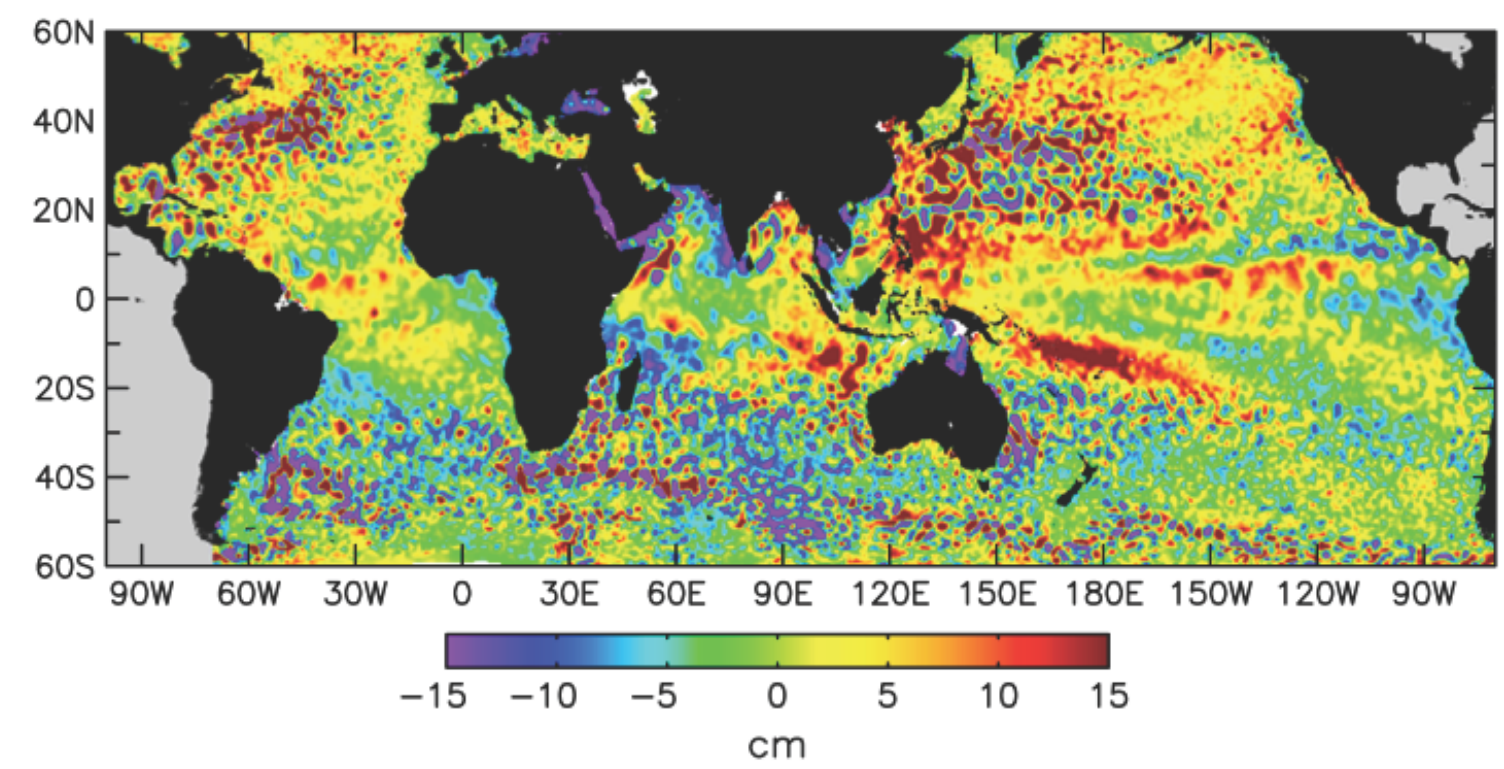

Figure 1-1: The global distribution of sea surface height on August 28, 1996 from the altimeter data. From Chelton et al. (2011).

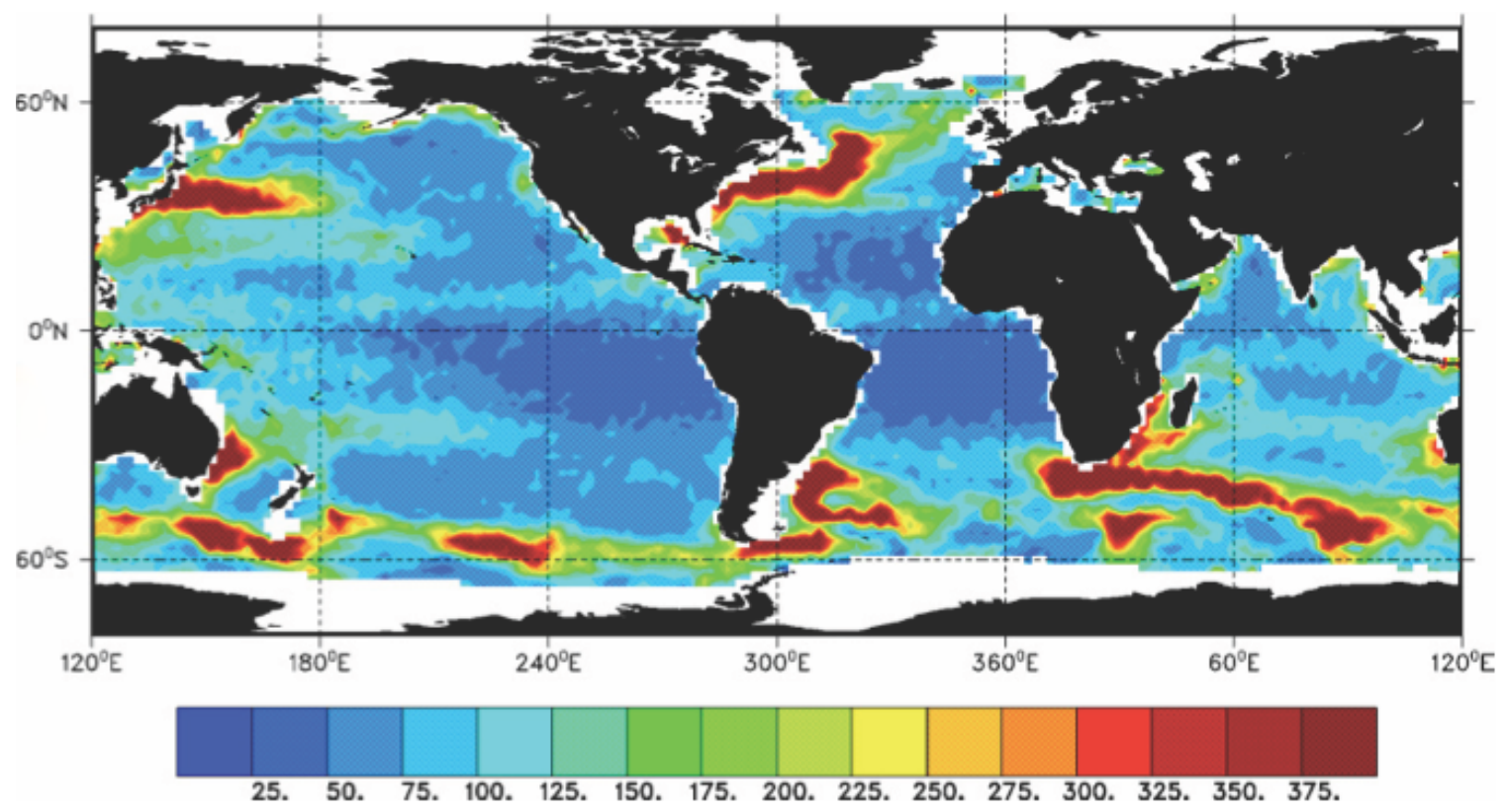

Figure 1-2: The surface geostrophic kinetic energy of oceanic variability multiplied by $\sin ^{2} \phi$ in $(\mathrm{cm} / \mathrm{s})^{2}$, where $\phi$ is the latitude. This estimate is based on the altimeter data. From Wunsch and Stammer (1998). 
dense water mass properties and variability there and the meridional overturning circulation. Another example is that eddies exchange energy with the time-mean circulation through eddy-mean flow interaction (e.g. Marshall, 1984; Plumb and Ferrari, 2005). The conversion rate from the time-mean circulation to eddy kinetic energy through baroclinic instability is roughly one third of the total wind power input into geostrophic flows (Wunsch, 1998; Ferrari and Wunsch, 2009).

Second, the short-term and accurate prediction of oceanic eddies can guide us in how to deal with the natural or human-induced disasters. For example, eddies can influence the intensity of hurricanes (e.g. Wu et al., 2007; Shay et al., 2000; Hong et al., 2000). Eddies, as an important part of oceanic motions, greatly influence the pathways of pollutants, such as oil from the massive spill in the Gulf of Mexico and nuclear waste. Thus, eddy prediction is crucial for the prediction of pollutant pathways.

Third, climate change can greatly affect human life, through influencing atmospheric temperature, sea ice coverage, global sea level, and the ocean ecosystem. Thus, accurate climate prediction is urgently needed to enhance our ability to respond to climate change. Recent developments in climate models show that increasing the resolution of ocean models to eddy-permitting levels can improve climate simulations (Delworth et al., 2012). This result indicates that oceanic eddies are one of the crucial components of the climate system and investigating them is a key to improving the long-term prediction of climate variability and related policy decisions. Here are some examples of specific reasons why understanding eddies is important for climate simulations:

- Atmospheric temperature is greatly influenced by the ocean through meridional oceanic heat transport and air-sea interaction. Eddies contribute significantly 
to the meridional heat transport (Volkov et al., 2010). The air-sea exchange of heat, momentum, and greenhouse gases takes place in the mixed layer. Reasonable representations of mixed layer eddies can reduce biases of mixed layer depth, and consequently can probably increase the model fidelity in the aspect of air-sea interaction (Fox-Kemper et al., 2008, 2011).

- The ocean has enormous heat capacity, and the slow component of the climate response to the greenhouse gas increase depends on the efficacy of ocean heat uptake (Gregory, 2000; Held et al., 2010). At high latitudes, mixing and convection processes transfer heat from the surface down to the deep ocean, and the globally integrated ocean heat uptake in climate models is sensitive to mixing parameterizations (Boé et al., 2009; Gregory, 2000; Huang et al., 2003). Eddy dissipation can sustain oceanic mixing (Ferrari and Wunsch, 2009); thus resolving or properly parameterizing eddy dissipation processes is essential to accurately estimating the ocean heat uptake and, ultimately, climate variability.

Fourth, because eddies greatly influence the ocean ecosystem, investigating eddies is essential to understanding the ocean ecosystem's response to climate variability. For example, eddies can induce strong vertical motions, and the resulting vertical advection of nutrients contributes significantly to the nutrient budget of the euphotic zone; eddies can also affect the exposure of biomass to light by moving biomass vertically in the light-varying field (Flierl and McGillicuddy, 2002).

Finally, from a public policy perspective, regional sea-level trends are more relevant than the globally averaged sea-level change, as our response to climate change should be city-specific. Advection, mixing and surface exchanges all affect the steric sea level, and advection contributes to more than half of the low-frequency variability 
of steric sea level in many regions (Piecuch and Ponte, 2011). We speculate that eddies play a non-negligible role in advection and thus investigating eddies is useful for predicting regional sea-level trends.

\section{$1.2 \quad$ Research problems}

Given the importance of oceanic eddies in various aspects, as discussed above, a great deal of effort has been made towards understanding oceanic eddies. Some examples of such efforts are characterizing the properties of coherent structures in the eddy field using altimeter data (e.g. Chelton et al., 2007; Xu et al., 2011), formulating and improving eddy parameterization schemes (e.g. Gent and Mcwilliams, 1990; FoxKemper and Menemenlis, 2008), developing and improving observation techniques, such as the wide-swath altimeter (e.g. Fu et al., 2010), and investigating mechanisms for the damping of mesoscale eddies, such as radiation of Rossby waves, frontogenesis and lee internal waves (e.g. Flierl, 1984; Capet et al., 2008; Nikurashin and Ferrari, 2010).

Though extensively studied, there are still many open problems about oceanic eddies for us to tackle before we can fully understand them and their implications, and can predict the variability of the oceanic circulation and the climate system accurately. This thesis focuses on two topics related to oceanic eddies: eddy-mean flow interaction and banded structures in the low-frequency component of the eddy field.

\subsubsection{Eddy-mean flow interaction}

The energy exchange between eddies and mean flow is an integral component of the

global energy pathway, starting from the external forcing (e.g. wind stress) and ending 
at dissipation at molecular scales (Ferrari and Wunsch 2010). Studying eddy-mean flow interaction is useful in understanding the generation/damping of oceanic eddies and thus oceanic mixing. Chapter 3 focuses on eddy-mean flow interaction.

Baroclinic instability, introduced by Charney (1947) and Eady (1949), is one type of eddy-mean flow interaction. It has been identified as an important eddy generation mechanism in the ocean (Gill et al., 1974; Robinson and McWilliams, 1974). Quite a few recent studies about eddy dynamics are based on the local baroclinic instability hypothesis (e.g. Held and Larichev, 1996; Tulloch et al., 2011; Panetta, 1993; Venaille et al., 2011; Arbic and Flierl, 2004), and these investigations suggest that this hypothesis is useful in many contexts. For example, Smith (2007) and Tulloch et al. (2011) found that the eddy scale with the fastest growth rate, based on the local linear instability analysis, is smaller than the observed eddy scales and they concluded that inverse cascade is important in setting the observed eddy scales.

To our best knowledge, there is no global survey about how well eddy-mean flow interaction in the ocean resembles the local baroclinic instability process from the energy perspective. Exploration in this aspect may help make the gap between the ocean and theories smaller. The local baroclinic instability hypothesis assumes that the ocean region is homogeneous and thus the energy released from the baroclinically unstable mean flow is used to sustain the eddy growth in the same region. However, the ocean is more complicated in many aspects. For example, some ocean regions are very inhomogeneous, indicating that some energy from the mean flow may be exported elsewhere through the divergence term, rather than being transferred to eddies in the same region (e.g. Kundu and Cohen, 2004). Also, in some regions, such as the Southern Ocean, mean flow can gain kinetic energy through eddy-mean flow interaction (e.g. McWilliams et al., 1978; Marshall, 1984; Johnson et al., 1992). ${ }^{1}$

\footnotetext{
${ }^{1}$ Some other studies about eddy-mean flow interaction are summarized in Section 3.1.
} 
Motivated by the lack of the global survey and recognizing that a description of the energy pathway is as important to our understanding about eddies as detailed process studies about energetics, we aim to describe eddy-mean flow interaction in an eddy-permitting model. Our specific aim is two-fold: mapping the respective change rate of energy in eddies and the mean flow through eddy-mean flow interaction in the global ocean; testing the local hypothesis from the energy perspective in the energetic ocean regions.

\subsubsection{Banded structures in the low-frequency eddy field}

The temporal average of the eddy field has banded structures in many ocean regions (Figure 1-3). This feature has been identified in both observations and numerical

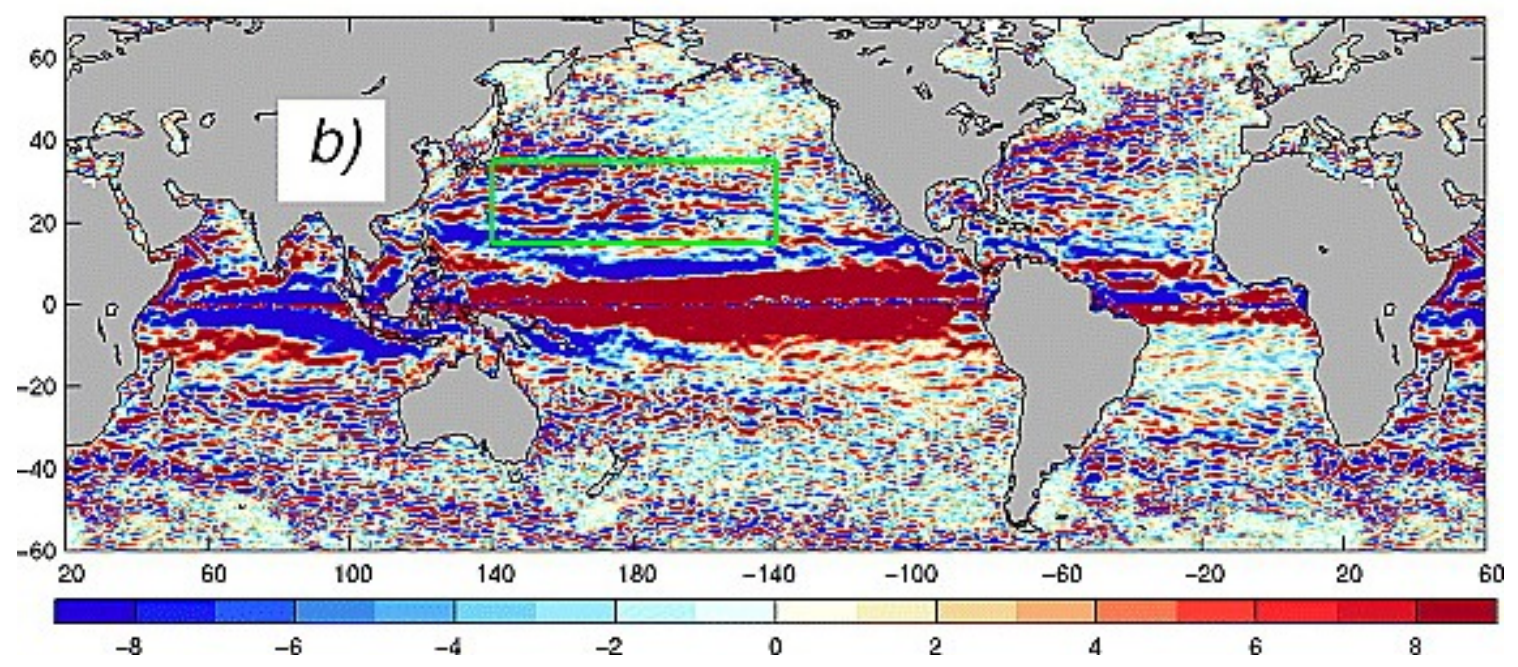

Figure 1-3: The 18-week average of the zonal geostrophic velocity anomaly in $\mathrm{cm} / \mathrm{s}$ at the ocean surface, calculated from the altimeter data. From Maximenko et al. (2005).

simulations (e.g. Cox, 1987; Maximenko et al., 2005; Richards et al., 2006) and it is termed "striations". Chapters 4 and 5 focus on the amplitude, origin and consequences of striations. 
Though striations have attracted much theoretical attention recently, it is still unclear how important striations are. For example, whether striations contribute significantly to energy pathways, tracer mixing and thus the climate mean state is to be assessed. A related question is how much oceanic variability is contained in striations. If the percentage is noticeable, the consequences of striations on the energy pathway and mixing are probably not negligible. We aim to explore these questions. To solve the related technical issues, we also provide a criterion to extract striations from the eddy field and formulate diagnostic framework to evaluate striations' consequences on tracer mixing and energy budgets.

Besides striations' amplitude and consequences, we also investigate the effect of time-mean circulation on striations' origin and direction. The formation mechanism of striations is still under debate and several hypotheses have been proposed. For example, banded structures can arise in the two-dimensional turbulence on a beta plane due to the arrest of inverse cascade by beta effect (Rhines, 1975, 1979); they can also arise as an averaging effect of many coherent vortices propagating westward (Schlax and Chelton, 2008). Some other mechanisms are beta plumes (e.g. Afanasyev et al., 2011), radiating instabilities of the eastern boundary current (e.g. Hristova et al., 2008; Wang et al., 2012), stationary Rossby waves (e.g. Maximenko et al., 2008), and the nonlinear self-interactions of the linear eigenmodes (e.g. Berloff, 2005). The origin of striations in the ocean is complicated, as many factors in the ocean can possibly influence striations, such as stratification and the large-scale wind forcing. One important fact ignored in the previous studies listed above is that striations are embedded in the large-scale time-mean circulation (Figure 1-4). There are subtropical and subpolar gyres in the Atlantic and Pacific Oceans, and the Antarctica Circumpolar Current (ACC) in the Southern Ocean. The direction of the time-mean circulation at a given location can be zonal, meridional, or in other directions. Since 


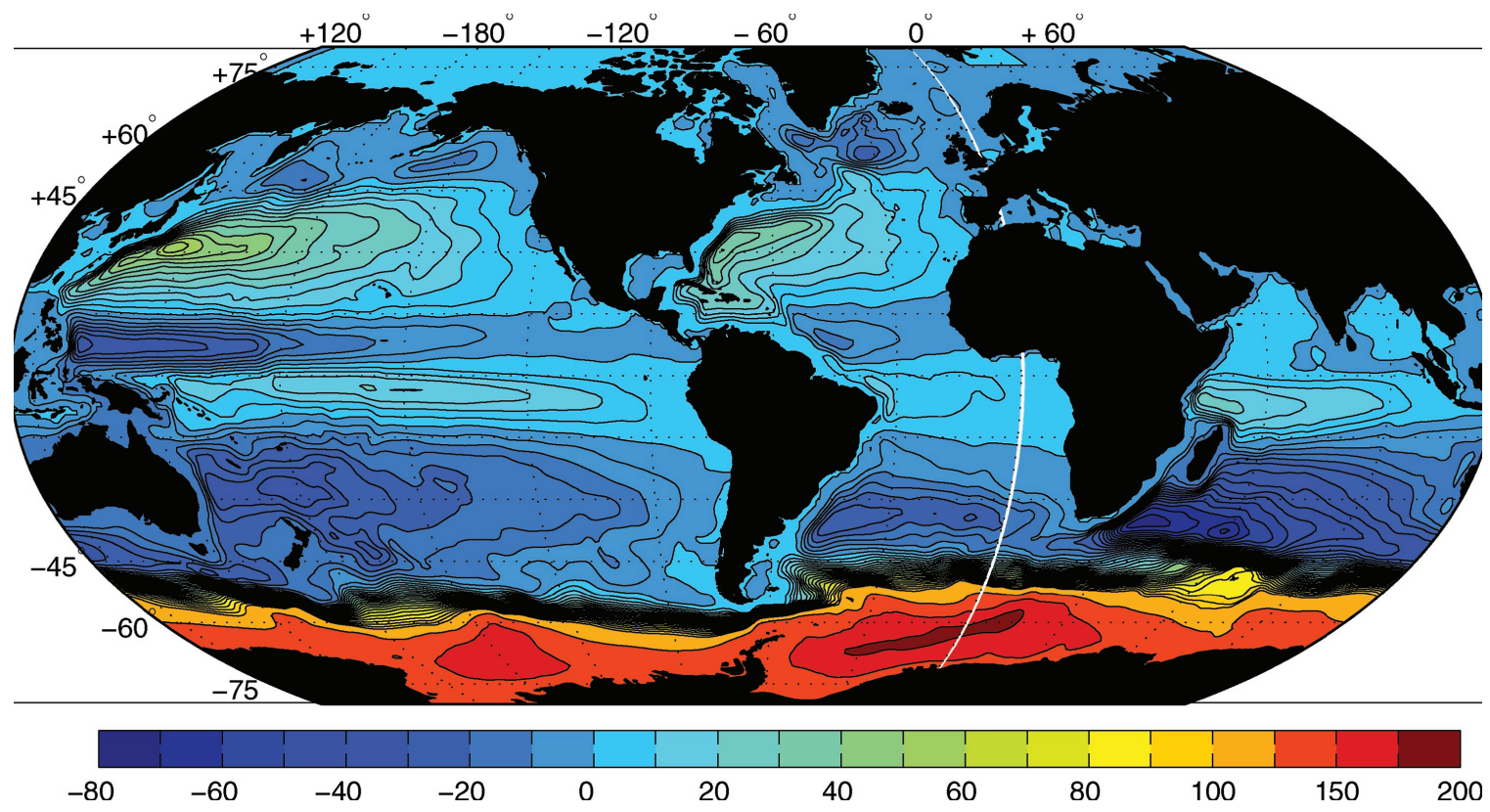

Figure 1-4: The 16-year average of the transport stream function in $10^{6} \mathrm{~m}^{3} / \mathrm{s}$. From Wunsch (2011).

both striations and the large-scale time-mean circulation are pervasive in the ocean, the effect of time-mean circulation on the origin and properties of striations is a relevant research problem. We present our results about this question in part of Chapters 4 and 5 .

\subsection{Methodology and outline}

The Estimating the Circulation and Climate of the Ocean, phase II: high resolution global-ocean and sea-ice data synthesis (ECCO2) state estimate is an important tool in this thesis research. There are quite a few basin and global eddy-permitting models, such as NLOM, POP, OFES and HYCOM (McClean et al., 2008). Numerical models have many uncertainties, such as the bottom drag coefficient. Compared to other eddying models, the advantage of the ECCO2 state estimate is that it is a forward run using the optimized values of these uncertain parameters, which are calculated by 
reducing the model-data misfit using the Green function approach. Thus, the ECCO2 state estimate is both realistic and dynamically consistent (Menemenlis et al., 2008).

Besides the ECCO2 state estimate, we also use simplified ocean models in this thesis. We estimate the percentage of oceanic variability associated with striations in the ECCO2 state estimate and then formulate/test the diagnostic framework about striations' consequences on mixing and energy pathway using a simple model. We also characterize striations in the subtropical gyre in the ECCO2 state estimate and then explain these characteristics using a 1.5-layer model. The ECCO2 state estimate guarantees that the research problems are relevant and helps identify key dynamical factors used to build up simple models. The simple models can provide dynamical insights due to the ease of interpretation and are useful in framework testing due to their computational efficiency.

This thesis is organized as follows. Chapter 2 describes and evaluates the ECCO2 state estimate. Chapter 3 characterizes the eddy-mean flow interaction in the global ocean from the energy perspective using the ECCO2 state estimate. Chapter 4 estimates the percentage of variability contained in striations using the altimeter data and the ECCO2 state estimate, formulates an idealized model and then uses it to assess the effect of a subtropical gyre on the origin and direction of striations. Chapter 5 formulates the diagnostic framework for evaluating the consequences of striations on tracer mixing and energy budget, and then illustrates their utility using a barotropic model. Chapter 6 summarizes the key findings and discusses remaining issues. 


\section{Chapter 2}

\section{The description of the ECCO2 state estimate}

\section{$2.1 \quad$ Introduction}

The Estimating the Circulation and Climate of the Ocean, phase II: high resolution global-ocean and sea-ice data synthesis (ECCO2) project aims to produce a physically consistent state estimate for the ocean circulation and sea ice. Two types of state estimation methods are employed in the ECCO2 project: the adjoint method (Wunsch and Heimbach, 2007) and the Green function method (Menemenlis et al., 2005a). The best available eddy-permitting solution at the time the author did her research was the cube87 version of the CS510 runs using the Green function approach. This solution is least-squares fit to observations. References describing this solution are Menemenlis et al. (2008, 2005a,b). Information about this solution and the ECCO2 project is also available at www.ecco2.org.

In this thesis, diagnosing the ECCO2 state estimate serves as an important methodology for the study of eddy-mean flow interaction and striations. There have been 
several previous successful studies about eddies using the ECCO2 state estimate. For example, $\mathrm{Fu}(2009)$ found that the eddy propagation velocity at the surface from the altimetry is consistent with those from the $\mathrm{ECCO} 2$ state estimate, indicating that the model captures well the eddy dynamics at the ocean surface. Volkov et al. (2008) and Volkov and $\mathrm{Fu}(2008)$ demonstrated that the ECCO2 state estimate is useful for the investigation of eddies and the oceanic heat transport variability in the Argentine Basin in the South Atlantic Ocean.

Though there are publications and information online about the ECCO2 state estimate using the Green function approach, here I give an elementary introduction about the state estimate for two purposes: provide enough background about the model configuration, state estimation method and observations for the convenience of the reader; discuss why the model solution is useful to study oceanic eddies. This chapter is organized as follows. Section 2.2 describes the model configuration, including surface/bottom boundary conditions, grids and resolution. Section 2.3 is about the state estimation method, observations used for the state estimation and the choice of control parameters. The model-data comparison shows that the model solution is overall very realistic (Section 2.4). Section 2.5 is the summary and discussion.

\subsection{Model configuration}

The ECCO2 project uses the Massachusetts Institute of Technology ocean general circulation model (Marshall et al., 1997a,b). The ocean model is coupled with an interactive sea ice model (Menemenlis et al., 2008); however, the discussion of sea ice is beyond the scope of this dissertation. Eq. 2.1-Eq. 2.7 are the governing equations for the ocean model. 


$$
\begin{aligned}
& \frac{\partial u}{\partial t}+\frac{\partial u u}{\partial x}+\frac{\partial u v}{\partial y}+\frac{\partial u w}{\partial z}-f v=-\frac{1}{\rho_{0}} \frac{\partial p}{\partial x}+\frac{\partial}{\partial z} A_{z} \frac{\partial u}{\partial z}+A_{4} \nabla_{h}^{4} u \\
& \frac{\partial v}{\partial t}+\frac{\partial v u}{\partial x}+\frac{\partial v v}{\partial y}+\frac{\partial v w}{\partial z}+f u=-\frac{1}{\rho_{0}} \frac{\partial p}{\partial y}+\frac{\partial}{\partial z} A_{z} \frac{\partial v}{\partial z}+A_{4} \nabla_{h}^{4} v \\
& \frac{\partial u}{\partial x}+\frac{\partial v}{\partial y}+\frac{\partial w}{\partial z}=0 \\
& \frac{\partial p}{\partial z}=-\rho g \\
& \rho=\rho\left(S, \theta, p_{0}\right) \\
& \frac{\partial S}{\partial t}+\frac{\partial u S}{\partial x}+\frac{\partial v S}{\partial y}+\frac{\partial w S}{\partial z}-\frac{\partial}{\partial z} K_{z} \frac{\partial S}{\partial z}=\left\{\begin{array}{l}
F_{S}(\text { surface level) } \\
0 \text { (interior) }
\end{array}\right.
\end{aligned}
$$

where $u, v$, and $w$ are velocities, $f$ is the Coriolis parameter, $p$ is the pressure, $A_{z}$ is the vertical Laplacian viscosity, $A_{4}$ is the horizontal biharmonic viscosity, $\rho_{0}$ is the constant reference density $\left(1027.5 \mathrm{~kg} / \mathrm{m}^{3}\right.$ in this configuration), $\rho$ is the insitu density, $S$ is salinity, $\theta$ is potential temperature with surface as the reference level, and $K_{z}$ is vertical diffusivity. Using the equation of state in Jackett and McDougall (1995), the model calculates density $\rho$ from potential temperature $\theta$, salinity $S$ and the constant pressure $p_{0}$, where $p_{0}=-g \rho_{0} z$ and $\mathrm{z}$ is water depth. $F_{\theta}$ and $F_{S}$ are surface forcing defined in Eqs. 2.8 and 2.9. The momentum equation used in the model configuration is the vector invariant form of Eqs. 2.1 and 2.2.

\section{Spatial domain and grids}

The model solution is from a free forward run on the entire globe with a finite volume discretization and 20-minute time step; it is saved at a three-day time interval and covers the period of 1992-2007. The longitude-latitude grid can be problematic 
for global simulations, as it produces two singularities at the poles; the cube-sphere grid avoids the singularity problems (Adcroft et al., 2004). Thus, the model employs a cube-sphere grid (Adcroft et al., 2004): the entire sphere is divided into six parts, with a $510 \times 510$ grid on each side (Figure $2-1$ ). The model uses a z-coordinate vertical discretization and has 50 vertical levels. The mean horizontal resolution is roughly $18 \mathrm{~km}$ and the vertical resolution decreases from $10 \mathrm{~m}$ at the surface to $456 \mathrm{~m}$ at the maximum model depth (Figure 2-1). The model uses General Bathymetric Charts of the Ocean for the topography in the Arctic Ocean and uses the bathymetry data from Smith and Sandwell (1997) for the rest of the ocean (Menemenlis et al., 2008).
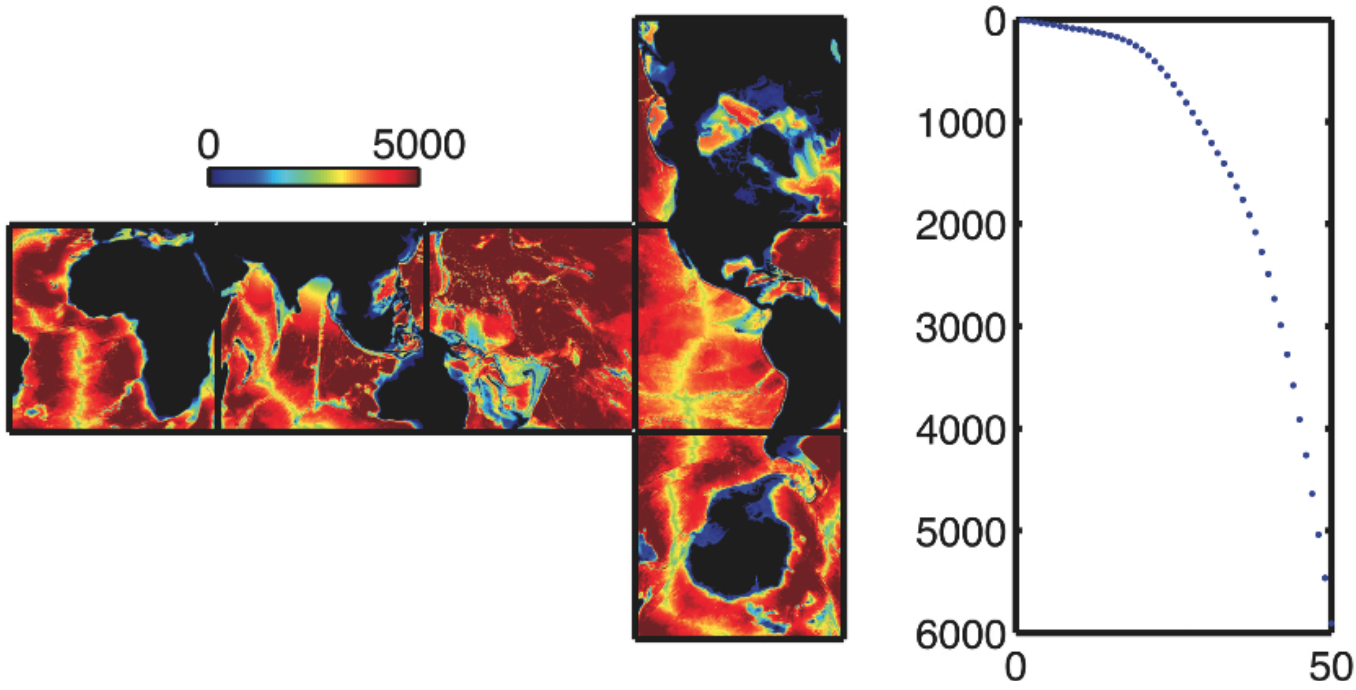

Figure 2-1: Left panel: the global bathymetry in $m$ on the cube-sphere grids used in the model. Right panel: the depth of levels (y axis) in $m$ as a function of level (x axis).

\section{Model assumptions and boundary conditions}

The Boussinesq approximation (Eq. 2.3) and the hydrostatic assumption (Eq. 2.4) are used in the model run. Given that the spatial resolution of this eddy-permitting model is $18 \mathrm{~km}$ on average, it is reasonable to use the hydrostatic assumption. The model uses slip boundary condition at the lateral boundaries and no-slip boundary 
condition at the ocean bottom. Physical processes at the bottom of the ocean are very complicated, involving internal waves and bottom boundary layer dynamics, etc. The model parameterizes these processes with the quadratic drag law:

$$
\tau_{b}=-\rho_{0} C_{d}|\mathbf{u}|_{b} \mathbf{u}_{b}
$$

where $\tau_{b}$ is the bottom stress, $\mathbf{u}_{b}$ is the bottom velocity and $C_{d}=0.0021$.

Surface forcing for potential temperature and salinity in Eqs. 2.6 and 2.7 follows

$$
F_{\theta}=\frac{Q_{n e t}-Q_{s w}}{C_{p} g \rho_{0} \delta z}+\frac{Q_{s w}}{C_{p} g \rho_{0} \delta z_{1}}+\text { non-local transport term }
$$

and

$$
F_{S}=-\frac{(E-P-R) S(x, y, t, z=0)}{\delta z}+\text { non-local transport term }
$$

where $Q_{n e t}$ is the net heat flux, $Q_{s w}$ is the short wave radiation, $C_{p}=3994 \mathrm{~J} /(\mathrm{C} \cdot \mathrm{kg})$, $g=9.81 \mathrm{~m} / \mathrm{s}^{2}, \rho_{0}=1027.5 \mathrm{~kg} / \mathrm{m}^{3}, \delta z$ is the depth of the first level, $\delta z_{1}$ is the penetration depth of shortwave radiation in the ocean (Paulson and Simpson, 1977), $E$ is the evaporation rate, $P$ is the precipitation rate, $R$ represents the river runoff and $S(x, y, t, z=0)$ is the local surface salinity. Non-local transport terms represent the temperature/salinity change due to the entrainment process parameterized using the K-profile parameterization (KPP) mixing scheme (Large et al., 1994).

\section{Subgrid scale parameterizations}

Current computers are not capable of resolving dissipation at molecular scales for simulations in the global domain. To make the model solution realistic, choosing the right sub-grid scale parameterization is important.

Biharmonic horizontal viscosity is used instead of the Laplacian horizontal viscosity in the ECCO2 state estimate. Biharmonic horizontal viscosity is determined 
by the modified version of the Leith scheme, which is a useful parameterization for simulations with gridscale smaller than the Rossby deformation radius (Fox-Kemper and Menemenlis, 2008). Explicit horizontal diffusivity is zero; however, the advection scheme is flux limited, which results in a numerically induced horizontal diffusivity.

The KPP scheme (Large et al., 1994) is used to parameterize unresolved vertical mixing processes, such as convection and double diffusion. In the ECCO2 state estimate, the vertical diffusivity and vertical viscosity change spatially and temporally, and they have the largest value in the KPP mixing layer. Note that the mixing layer is different from the mixed layer: the mixing layer depth is defined as the depth to which the KPP mixing penetrates at each time step, while the mixed layer is a result of the cumulated effect of mixing over a time interval. The mixing layer depth has large spatial and temporal variability: the daytime depth can be $5 \mathrm{~m}$ and the depth at convection events can be $2000 \mathrm{~m}$ or more (D. Menemenlis and H. Zhang 2012, personal communication). The background vertical viscosity is approximately $5.7 \times 10^{-4} \mathrm{~m}^{2} / \mathrm{s}$, but it is roughly $[5-100] \times 10^{-3} \mathrm{~m}^{2} / \mathrm{s}$ in the mixing layer. Another region with large vertical viscosity is the equatorial region, as shear instability penetrates below the ocean surface there.

\section{Forcing and initial conditions}

The ECCO2 solution is constrained to observations by adjusting several control parameters based on the model-data misfit (Table 2.1 and Table 2.2). The control parameters include initial conditions and atmospheric surface forcing; thus, the initial condition and atmospheric forcing are a blend of several products (Table 2.1). 


\subsection{The dynamically consistent state estimation method}

Model biases and drifts are very common in model simulations and we need to reduce them as much as possible in order to accurately simulate the ocean circulation. One advantage of the ECCO2 state estimate used in the thesis is that the solution is optimized and thus model drifts and biases are greatly reduced; however, the solution is still dynamically consistent. Next, we provide a heuristic description about the optimization method. Readers can refer to Menemenlis et al. (2005a) and Wunsch and Heimbach (2007) for a rigorous discussion.

\subsubsection{Green function method}

A general circulation model (GCM) solves the equations (Eq. 2.1-Eq. 2.7) through discretization. Though complicated, the GCM can be represented as

$$
X^{f}\left(t_{i+1}\right)=M_{i}\left[X^{f}\left(t_{i}, \eta\right)\right]
$$

where $X^{f}$ is the GCM state vector (temperature, salinity, velocity, etc.), and $t_{i}\left(t_{i+1}\right)$ is the discrete time in the model at time step $i(i+1) . M_{i}$ denotes the computing rules in the GCM, which can calculate the state vector at $t_{i+1}$ from that at previous time steps. $\eta$ represents the control parameters to be adjusted through the Green function method, such as initial conditions, viscosity, diffusivity and the bottom drag coefficient. In practice, there are more unknown parameters in a GCM than the ones that we choose to adjust and these are given fixed values based on experience or previous studies.

$Y^{o}$ denotes observations that are used in the state estimate to obtain the optimized 
values of $\eta$. It satisfies

$$
Y^{o}=G(\eta)+\epsilon
$$

where $G$ is the Green function and it is related to $M_{i}$. $G(\eta)$ are values from the model solution at the locations and time of observations. $\epsilon$ represents the difference between observations and modeled values, and it includes the measurement error, model solution errors and the interpolation error caused by mapping model solution to observation locations. There are many types of model solution errors: errors/assumptions in the governing equations (Eq. 2.1 -Eq. 2.7), such as hydrostatic approximation, the Boussinesq approximation and inaccurate parameterizations of subgrid scale processes; truncation errors from discretization; errors and uncertainties in initial conditions $X^{f}(t=0)$ and in surface forcing (e.g. wind stress and heat flux). These errors can propagate and contaminate the solution.

The Green function method minimizes the misfits between model and observations by using an optimized set of control parameters $\eta$. To obtain the optimized control parameters, first we linearize Eq. 2.11

$$
Y^{0}=G\left(\eta_{0}\right)+\left(\eta-\eta_{0}\right) \mathbf{G}+\epsilon,
$$

where $G\left(\eta_{0}\right)$ is the model solution at the data locations in the baseline experiment and $\eta_{0}$ is the control parameters used in the baseline solution. $\mathbf{G}$ can be obtained from a set of sensitivity experiments, in which the perturbed values of the control parameters are used (details are in Menemenlis et al., 2005a). A useful representation of the model-data misfit is the cost function

$$
J=\eta^{T} Q^{-1} \eta+\epsilon^{T} R^{-1} \epsilon,
$$


where $R$ are the covariances of observations (i.e. weights) and $Q$ are the covariances of $\eta$. The optimized control parameters $\left(\eta_{a}\right)$, which minimize the cost function $J$ given Eq. 2.12, can be obtained by solving the linear simultaneous equations. The solution is

$$
\eta_{a}=\eta_{0}+\left(Q^{-1}+\mathbf{G}^{T} R^{-1} \mathbf{G}\right)^{-1} \mathbf{G}^{T} R^{-1}\left[Y^{0}-G\left(\eta_{0}\right)\right] .
$$

The ECCO2 state estimate we use is essentially a free forward run using the optimized control parameters. Note that observations are only used to find the optimized control parameters and to compare with the model solution. Thus, the ECCO2 solution is dynamically consistent.

\subsubsection{Control parameters, observations and weights}

The number of sensitivity experiments needed to obtain $\mathbf{G}$ is directly proportional to the number of control parameters. Thus, only a small number of uncertain variables in the GCM are chosen as control parameters (Table 2.1). The one dimensional control parameters used in the baseline experiment are only slightly different from the optimized ones; however, the model-data misfit is greatly reduced by using the optimized ones (Figures 2-3, 2-4, and 2-5).

Observations used to determine the optimized control parameters include sea surface height (SSH), temperature, salinity and sea ice thickness/concentration during the period of 1992-2002 (Table 2.2). Temperature/salinity observations include CTD (conductivity, temperature and depth), XBT (expendable bathythermograph), Argo float profiles and TAO (tropical atmosphere ocean) profiles. The spatial pattern of the data distribution is shown in Figure 2-2. Overall, the hydrography measurements cover most of the region in the global ocean. However, there are not many ARGO measurements before the year 2003 from the mid-latitudes of the Pacific Ocean; the 


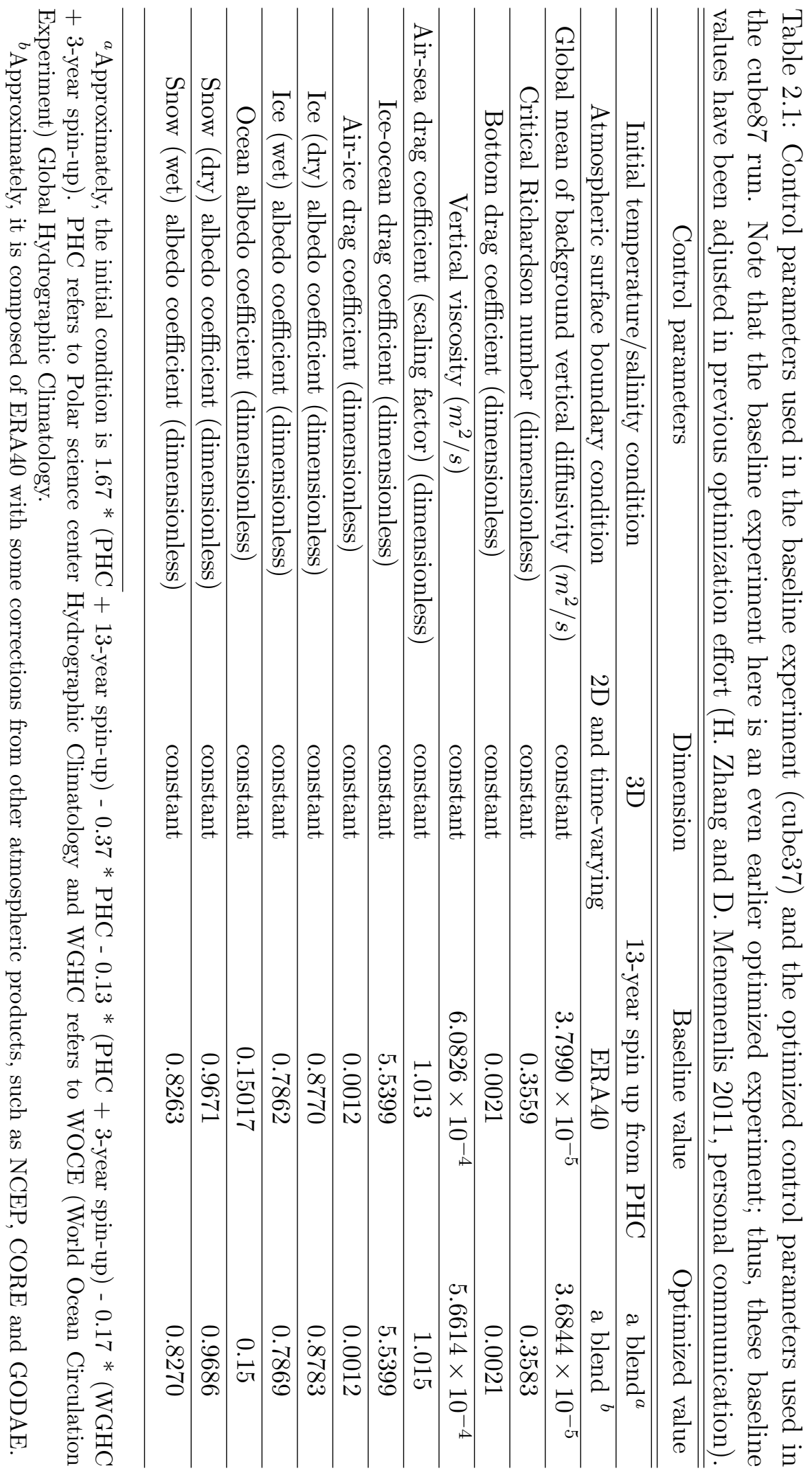


hydrography data in the polar region is also very sparse. The amount of available hydrography data generally decreases with depth. The XBT hydrography data used covers the upper $1000 \mathrm{~m}$; the ARGO hydrography data used covers the upper 2000 $m$; the TAO hydrography data used covers roughly the upper $800 \mathrm{~m}$. The CTD cross sections used extend to roughly $5500 \mathrm{~m}$ and are the only hydrography data to constrain the solution below $2000 \mathrm{~m}$; however, the CTD data is sparse horizontally (Figure 2-2).
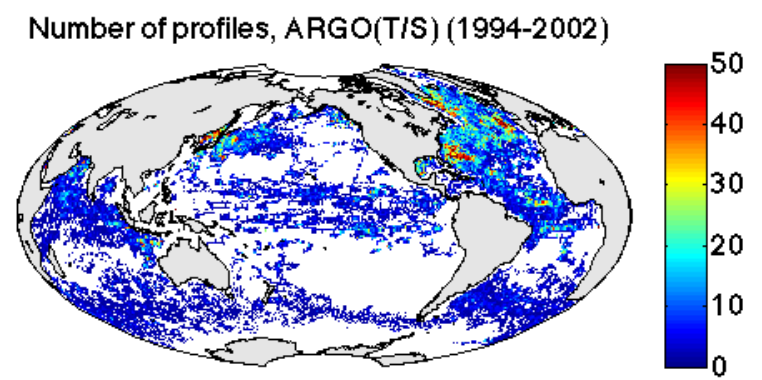

Number of profiles, CTD(T/S) (1992-2002)
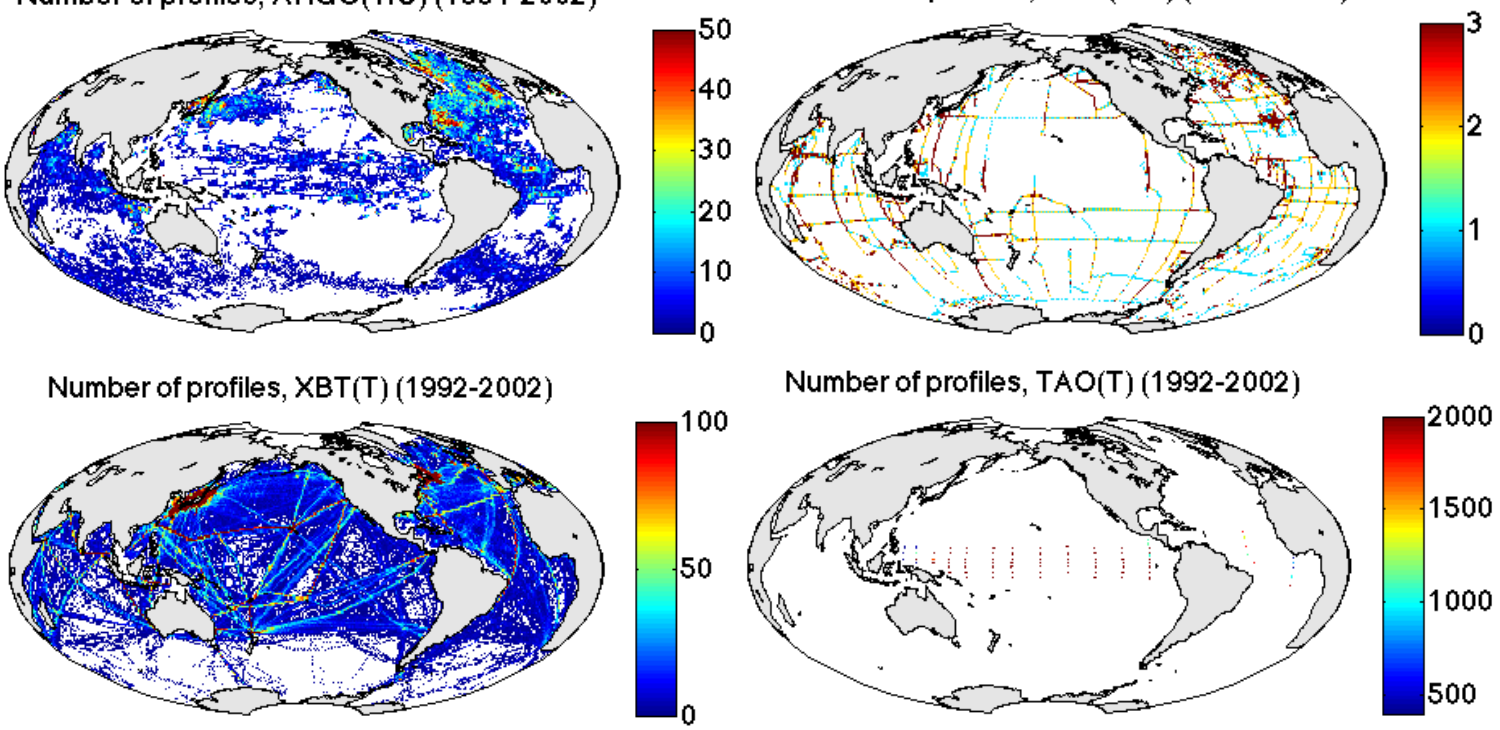

Number of profiles, TAO(T) (1992-2002)

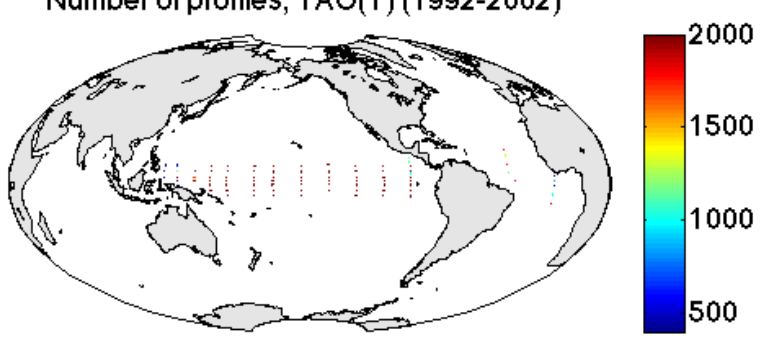

Number of profiles, TAO(S) (1992-2002)

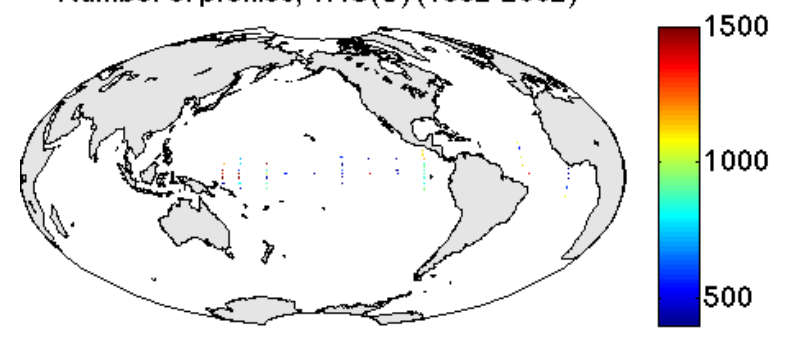

Figure 2-2: The number of observed vertical profiles in each $1^{\circ} \times 1^{o}$ grid box. Upper left: the number of temperature/salinity vertical profiles from ARGO (1994-2002); upper right: the number of temperature/salinity profiles from CTD (1992-2002); mid-left: the number of temperature vertical profiles from XBT (1992-2002); midright: the number of temperature vertical profiles from TAO (1992-2002); bottom: the number of salinity vertical profiles from TAO (1992-2002). 


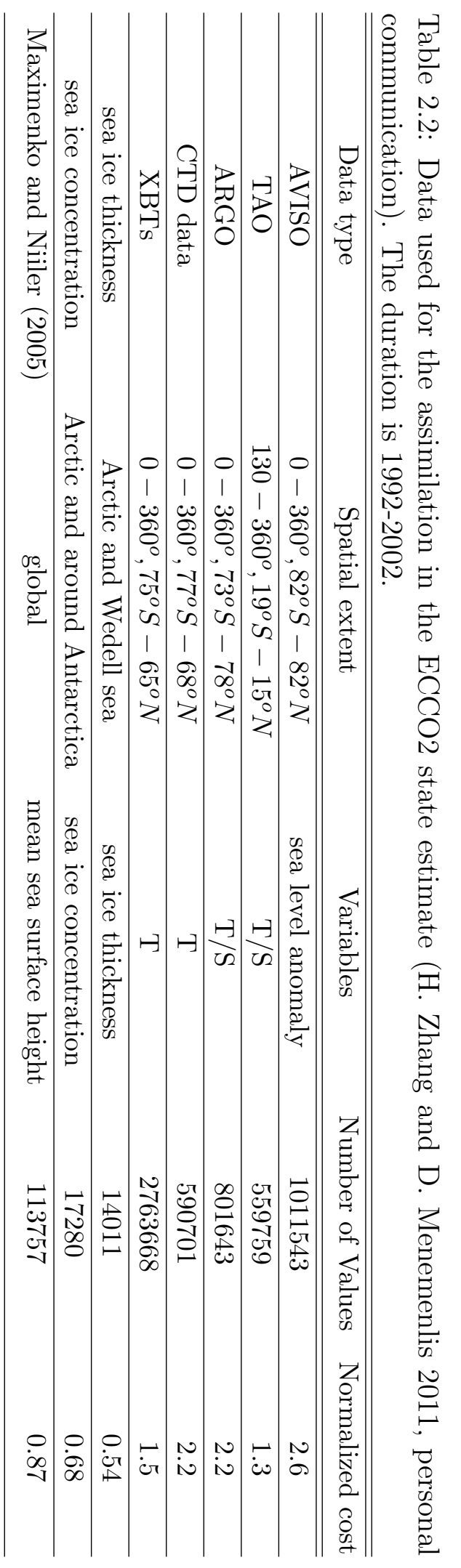


Besides observations, weights are needed to determine the optimized control parameters (Section 2.3.1). Each observation value has a corresponding weight. The weights for temperature observations (ARGO, XBT, TAO, CTD) are

$$
\left[\max \left(\sigma_{T}(i), \sigma_{T 0}\right)\right]^{-2}
$$

where $\sigma_{T 0}$ is a constant with small magnitude representing the instrument error. $\sigma_{T}(i)$ is the temperature standard deviation at the regular grid point closest to the location of the in-situ profile and it is from Forget and Wunsch (2007). The weights for salinity observations are determined in a similar way.

The model is overall consistent with the observations within the error bars. Table 2.2 shows the normalized cost for each dataset. The normalized cost is

$$
\text { Normalized cost }=\frac{1}{N} \sum_{i=1}^{N}\left[\operatorname{Var}_{\text {model }}(i)-\operatorname{Var}_{\text {observation }}(i)\right]^{2} \times \operatorname{Weight}(\operatorname{Var}, \mathrm{i}) \text {, }
$$

where $\operatorname{Var}_{\text {model }}$ is the variable in the model, such as sea surface height, temperature

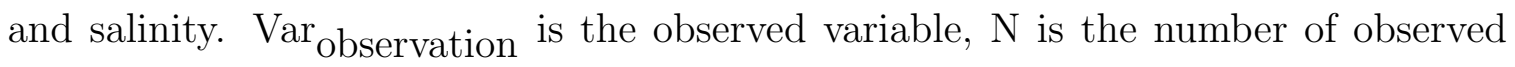
values of the particular dataset, and Weight(Var, i) is the corresponding weight for each observed value, which is discussed in the last paragraph. The normalized cost is an indicator of the model-data consistency. If the normalized cost is one, the model is consistent with observations within the error bars. However, if the normalized cost is larger than one, either the weights chosen are too small or the model-data misfit is large. The normalized costs shown in Table 2.2 range from 0.54 to 2.6, indicating that the state estimate is roughly consistent with observations. 


\subsection{Model-data comparison}

To assess whether the ECCO2 state estimate is realistic enough for the eddy study, the hydrography and eddy variability in the ECCO2 state estimate is compared with observations. Data sets used for the comparison include ARGO, XBT, WOA05 (Collier and Durack, 2006), sea surface height from AVISO during 1992-2007 (Dibarboure et al., 2009) and the hydrographic variability data (Forget and Wunsch, 2007). Most of these observations are used to get the optimized control parameters for the state estimate. Results here are incomplete; for example, the comparison between modeled and observed sea surface temperature has not been done.

\subsubsection{Comparison between modeled and observed hydrogra- phy}

The hydrography in both the baseline (cube37) and the optimized (cube87) experiments is compared with observations (ARGO, XBT, and WOA05). The misfit of the model state to the observed hydrography is reduced through the state estimation (Figures 2-3, 2-4 and 2-5).

Figure 2-3 shows the misfit of the model solution to the XBT and ARGO data. The temperature in the baseline experiment is higher than that from the XBT/ARGO profiles in most regions. Compared to the XBT temperature, the temperature in the optimized experiment is slightly lower in the subtropical gyres and slightly higher in the high latitudes of the North Pacific and North Atlantic. The misfit pattern of the optimized model state to the ARGO temperature is similar to that of the baseline model state, but with smaller magnitude. The salinity misfit is not reduced much through the optimization except in the equatorial Atlantic region. The salinity in the model is too high in the high latitudes of the North Atlantic and too low in the 
$\Delta T$ of baseline wrt XBT

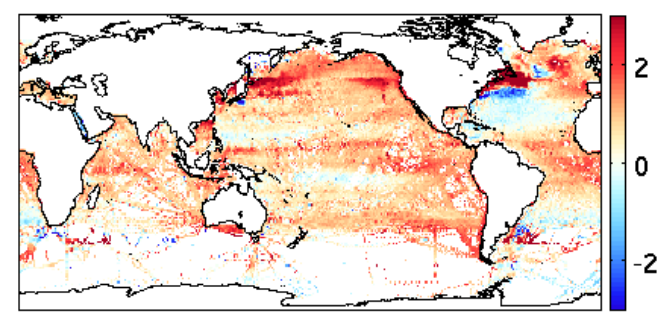

$\Delta T$ of baseline wit ARGO

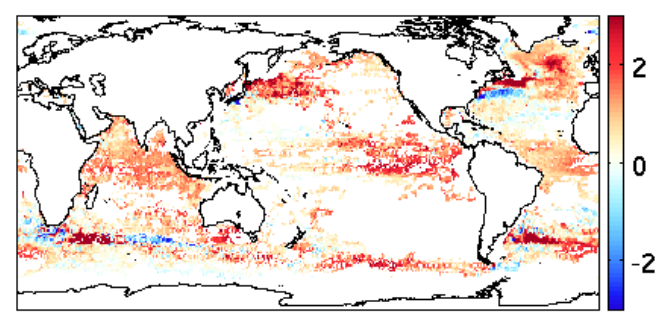

$\triangle \mathrm{S}$ of baseline wrt ARGO

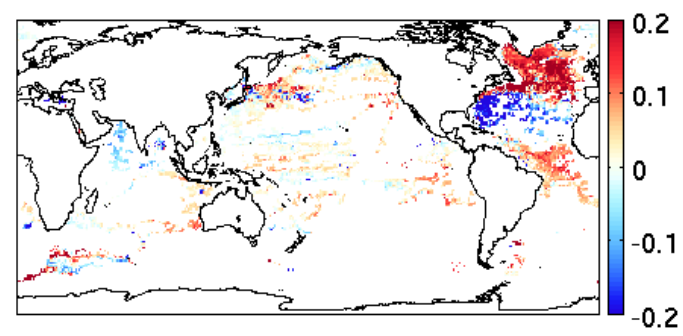

$\Delta T$ of optimized wrt XBT

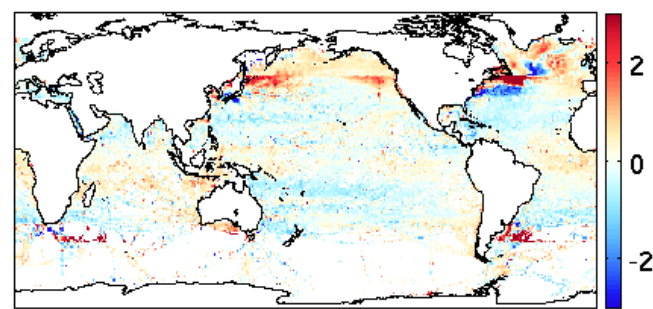

$\triangle T$ of optimized wrt ARGO

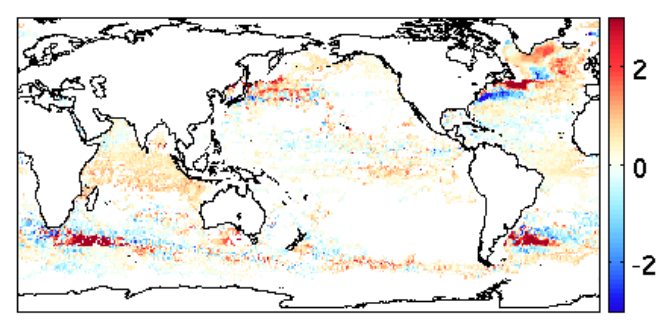

$\Delta S$ of optimized wit ARGO

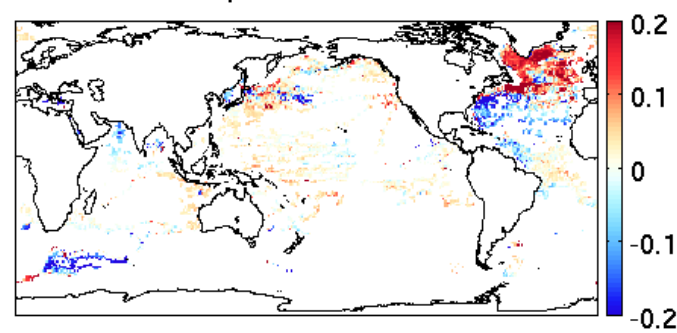

Figure 2-3: Left panel: the misfit of the baseline model state (cube37) to "XBT temperature" in ${ }^{\circ} \mathrm{C}$ (upper) , "ARGO temperature" in ${ }^{\circ} \mathrm{C}$ (middle) and "ARGO salinity" (bottom). Right panel: the misfit of the optimized model state (cube87) to "XBT temperature" in ${ }^{\circ} \mathrm{C}$ (upper), "ARGO temperature" in ${ }^{\circ} \mathrm{C}$ (middle) and "ARGO salinity" (bottom). "XBT temperature" refers to the temporally and vertically averaged temperature from XBT in the upper $1 \mathrm{~km}$ during 1992-2002. "ARGO temperature (salinity)" refers to the temporally and vertically averaged temperature (salinity) from ARGO in the upper $2 \mathrm{~km}$ during 1992-2002. From Hong Zhang and Dimitris Menemenlis.

subtropical gyre regions in the North Atlantic.

There are two notable features in the spatial pattern of the misfit (Figures 2-3, 2-4 and 2-5). First, the misfit of the model state in the optimized experiment to the observed hydrography is larger in the high latitudes than that in the mid- and 


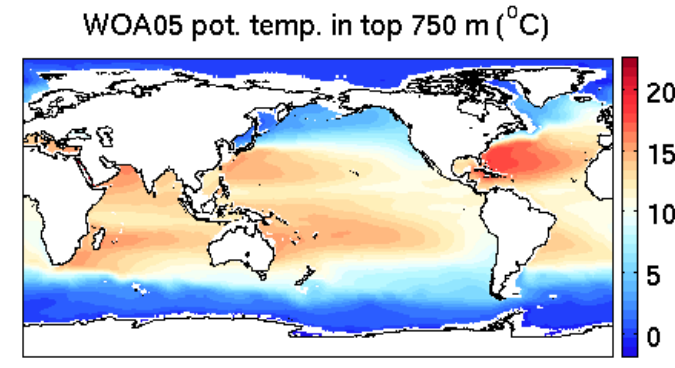

$\Delta T$ of baseline wrt WOA05

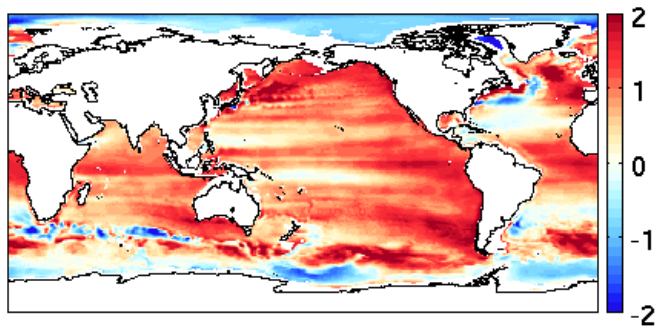

$\Delta \mathrm{T}$ of optimized wrt WOA05

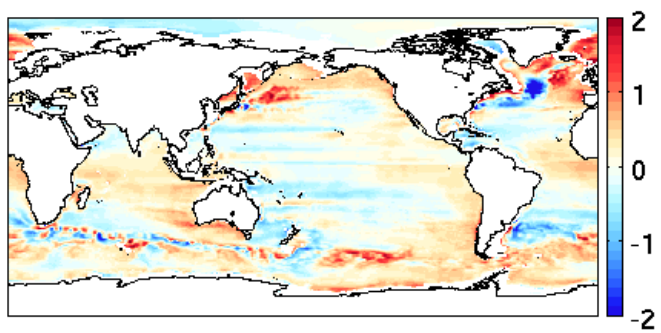

WOA05 salinity in top $750 \mathrm{~m}$ (PSU)

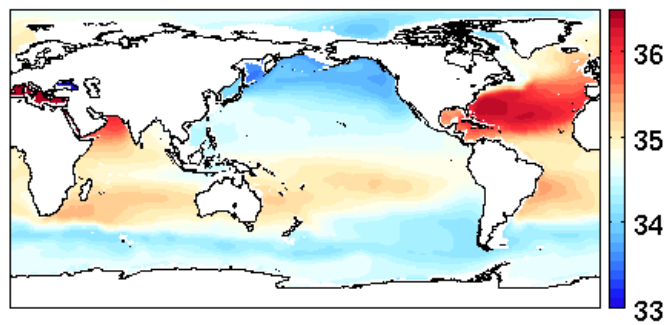

$\triangle S$ of baseline wrt WOA05

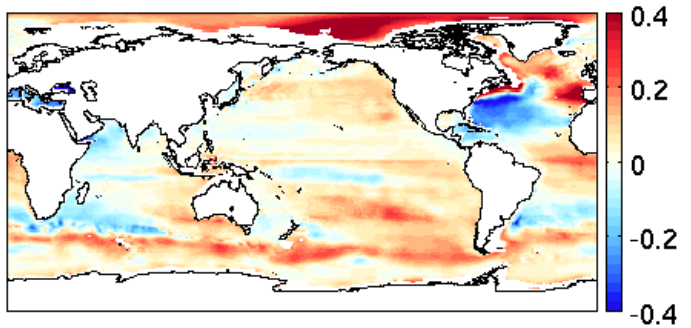

$\triangle S$ of optimized wrt WOA05

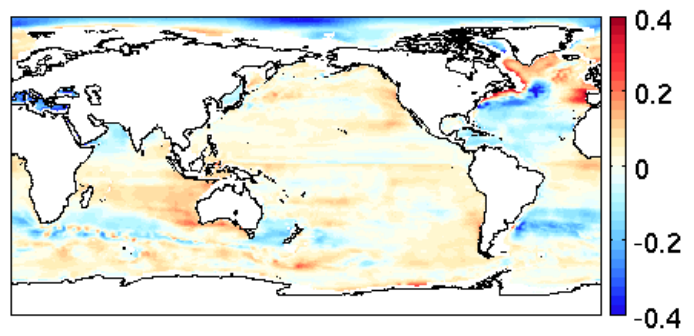

Figure 2-4: Upper panel: the vertical average of temperature in ${ }^{\circ} C$ (left) and salinity (right) over the upper $750 \mathrm{~m}$ from the WOA05 dataset. Middle panel: the misfit of the baseline model state (cube37) to the vertically averaged temperature in ${ }^{\circ} \mathrm{C}$ (left) and salinity (right) over the upper $750 \mathrm{~m}$ from the WOA05 dataset. Lower panel: the misfit of the optimized model state (cube87) to the vertically averaged temperature in ${ }^{\circ} \mathrm{C}$ (left) and salinity (right) over the upper $750 \mathrm{~m}$ from the WOA05 dataset. The model climatology is the temporal average over the year 1992-2002. From Hong Zhang and Dimitris Menemenlis.

low latitudes. Possible reasons are discussed in Section 2.4.2. Second, the misfit in the optimized experiment, such as the salinity misfit, is generally larger in the Atlantic Ocean than that in the Pacific Ocean. This suggests that the model has better performance in the regions without dense water formation and overflow. 


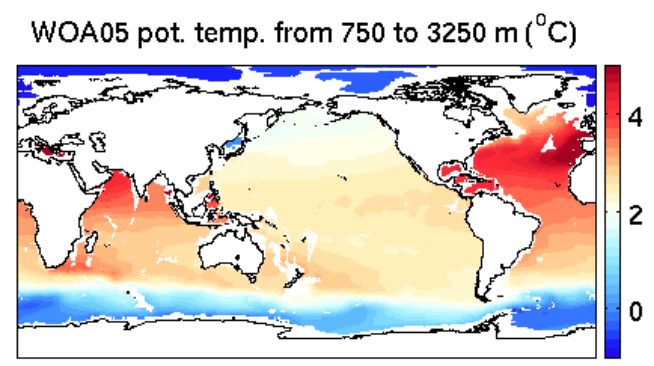

WOA05 salinity from 750 to $3250 \mathrm{~m}$ (PSU)
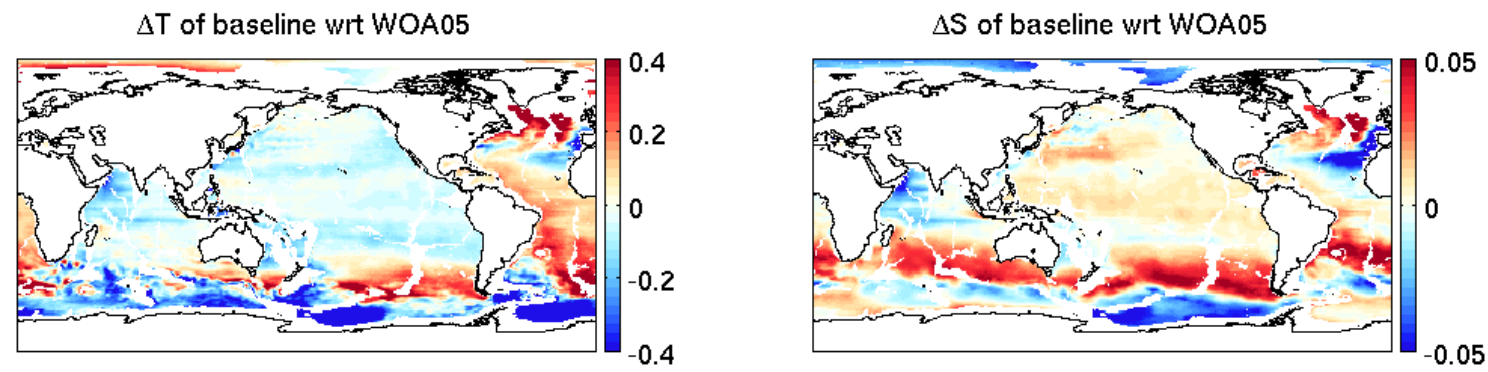

$\Delta T$ of optimized wrt WOA05
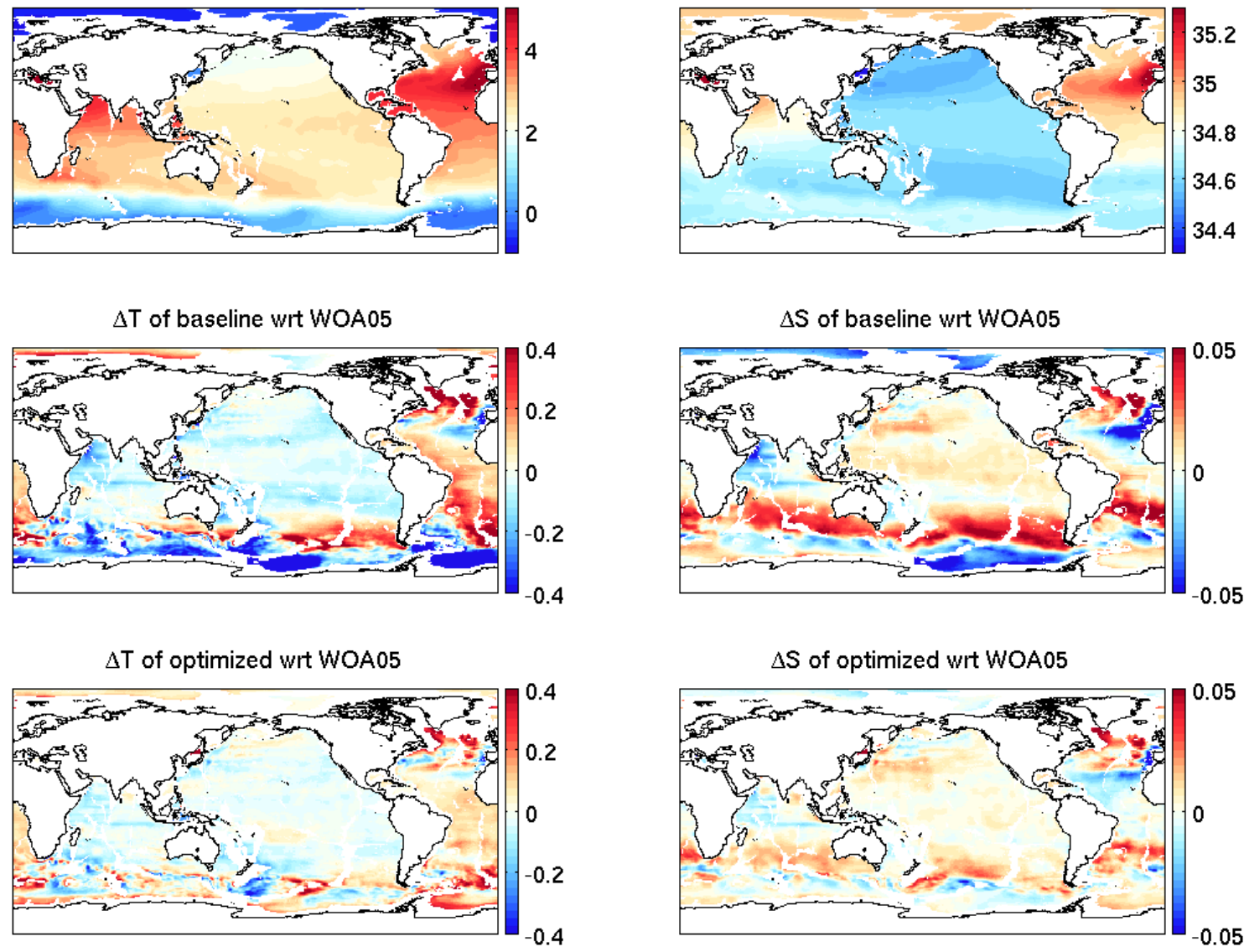

$\triangle S$ of optimized wrt WOA05

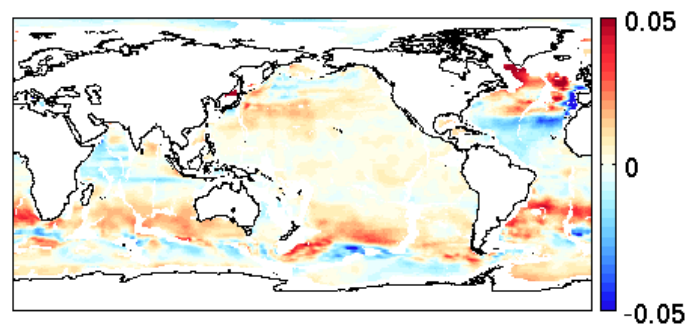

Figure 2-5: Upper panel: the vertical average of temperature in ${ }^{\circ} \mathrm{C}$ (left) and salinity (right) over $[750,3250] \mathrm{m}$ from the WOA05 dataset. Middle panel: the misfit of the baseline model state (cube37) to the vertical average of temperature in ${ }^{\circ} \mathrm{C}$ (left)/salinity (right) over $[750,3250] m$ from the WOA05 dataset. Lower panel: the misfit of the optimized model state to the vertical average of temperature in ${ }^{\circ} \mathrm{C}$ (left)/salinity (right) over $[750,3250] m$ from the WOA05 dataset. The model climatology is the temporal average over the year 1992-2002. From Hong Zhang and Dimitris Menemenlis.

\subsubsection{Comparison between modeled and observed eddies}

It is not realistic for the model to predict the location and properties of each vortex correctly. Thus, here we compare the spatial pattern and the order of magnitude of temporal variability in the optimized experiment (cube87 run) with those in observations. There has been no standard way to test the model fidelity quantitatively and 
here we choose an arbitrary standard: we consider the modeled eddies realistic, if the relative misfit

$$
\left|\sigma_{\text {observation }}-\sigma_{\text {model }}\right| / \sigma_{\text {observation }}<0.5
$$

where $\sigma_{\text {observation }}\left(\sigma_{\text {model }}\right)$ is the standard deviation of the observed (modeled) variable (e.g. SSH, T and S).

Figure 2-6 compares the observed SSH variability from AVISO with that in the model. The spatial pattern is qualitatively similar: the variability is large in the western boundary currents and the ACC; it is small in the eastern part of the basin. Most regions with large misfit are the regions with large variability. The model overestimates the variability in the Kuroshio Current, the Gulf Stream and the Agulhas ring region; but it underestimates the variability east of these two western boundary currents and the variability in the southwest part of the Indian Ocean. The relative misfit (lower right panel) is generally smaller than 0.5 in the mid- and low latitudes, but larger than 0.5 in the high latitudes. Consistently, the misfit of the modeled SSH to observations is also smaller in the mid- and low latitudes than high latitudes (Figures 2-7 and 2-8). The observed SSH is slightly lower than the modeled value near the Antarctica, in the northwestern part of the Pacific and the northernmost Atlantic Ocean; it is slightly higher in the subtropical region, especially in the region where the horizontal gradient of the thermocline depth is large.

Wortham (2012) compared the frequency-wavenumber spectrum of sea surface height variability in the ECCO2 state estimate with that from the altimeter data in a representative high energy region and a representative low-energy region. He found that the spectra in the model are qualitatively similar to the observed spectra in the two regions. This indicates that the simulated eddy-eddy interaction and eddy-mean flow interaction process in the model is reasonable, as the spectral structure is a result 
of external forcing and the nonlinear process in the ocean.
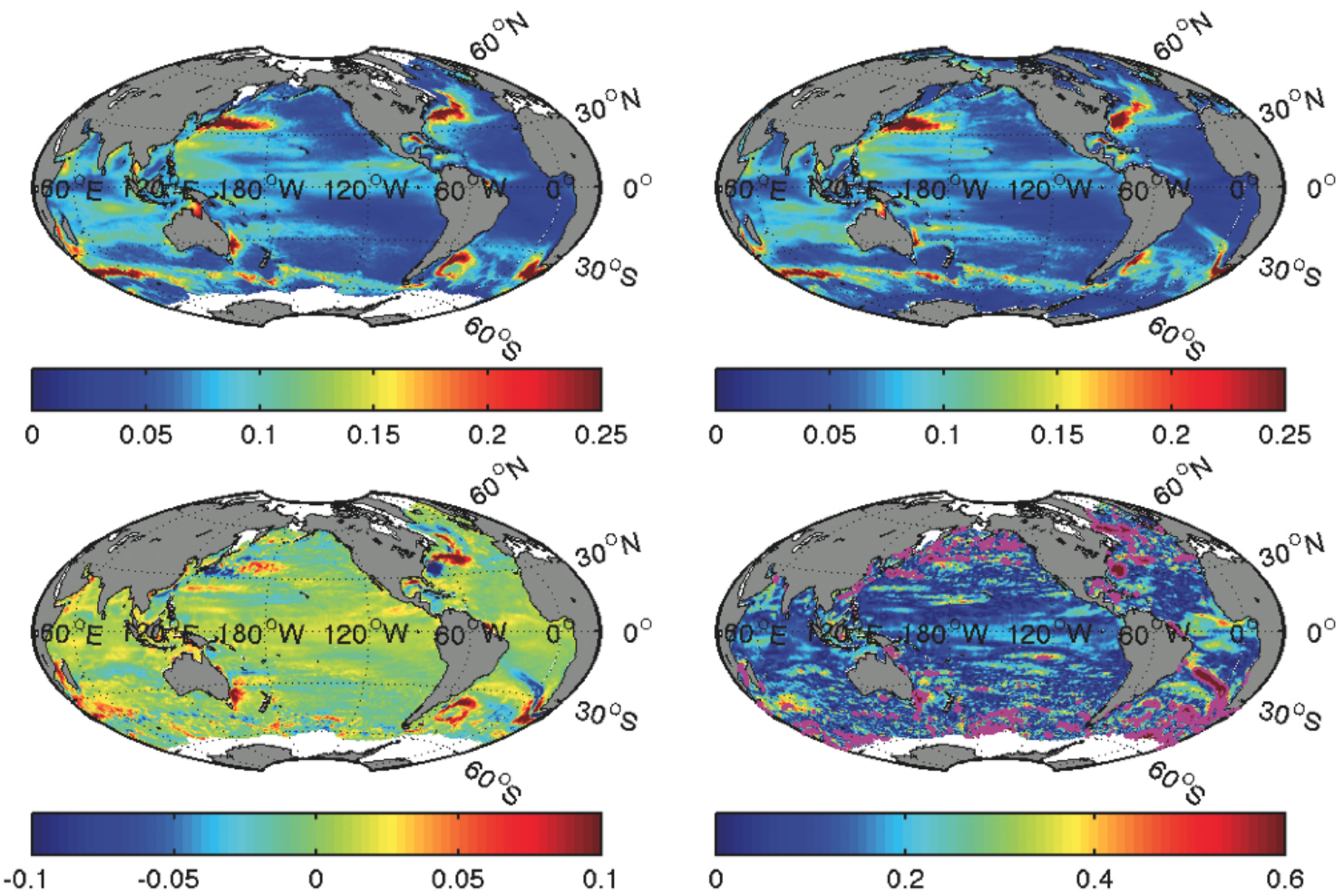

Figure 2-6: $\sigma_{S S H o}$ in $m$ (upper left), $\sigma_{S S H m}$ in $m$ (upper right), $\sigma_{S S H o}-\sigma_{S S H m}$ in $m$ (lower left) and the relative misfit $\left|\sigma_{S S H o}-\sigma_{S S H m}\right| / \sigma_{S S H o}$ (lower right). $\sigma_{S S H o}$ $\left(\sigma_{S S H m}\right)$ is the standard deviation of the observed (modeled) sea surface height over 1992-2007. Sea surface height here refers to the sea surface height relative to the global mean SSH at the same time step. Magenta contours in the lower right panel have the value of 0.5 .

Figures 2-9, 2-10 and 2-11 compare the temperature and salinity variability in the model with the observed variability from Forget and Wunsch (2007). The overall spatial features and the order of magnitude of hydrographic variability in the model are consistent with observations. For example, the variability at $250 \mathrm{~m}$ in both observations and the model is large in the Kuroshio Extension, the Gulf Stream Extension, the ACC and the thermocline; the relative misfit at $250 \mathrm{~m}$ is smaller than 0.5 in most regions, but it is larger than 0.5 in some regions in the Southern Ocean, the northernmost part of the Atlantic and some spots in the equatorial region (Figure 

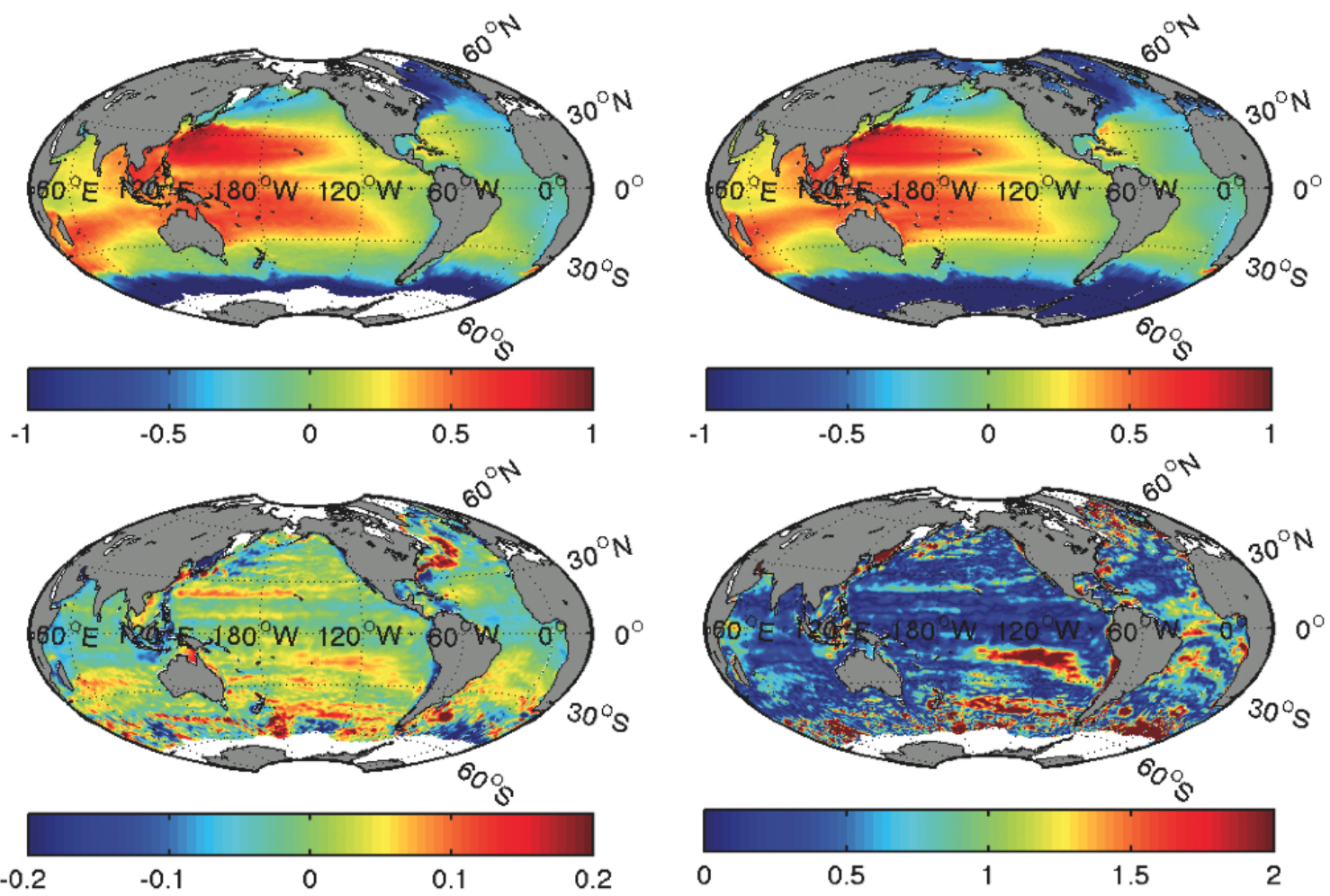

Figure 2-7: $\overline{S S H_{o}}$ in $m$ (upper left), $\overline{S S H_{m}}$ in $m$ (upper right), $\overline{S S H_{o}}-\overline{S S H_{m}}$ in $m$ (lower left) and $\left|\overline{S S H_{o}}-\overline{S S H_{m}}\right| / \sigma_{S S H_{o}}$ (lower right). $\overline{S S H_{o}}\left(\overline{S S H_{m}}\right)$ is the time mean of observed (modeled) sea surface height over 1992-2007 with global mean SSH subtracted.

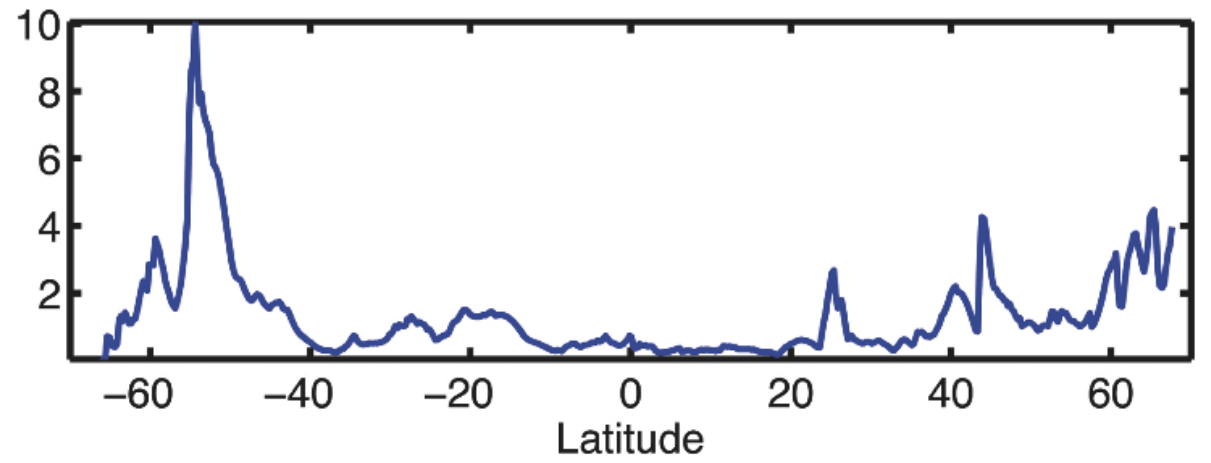

Figure 2-8: The zonal average of the normalized misfit $\left(\left|\overline{S S H_{o}}-\overline{S S H_{m}}\right|^{2} / \sigma_{S S H_{o}}^{2}\right)$.

$2-9)$.

Figure 2-10 shows the relative misfit of hydrographic variability as a function 

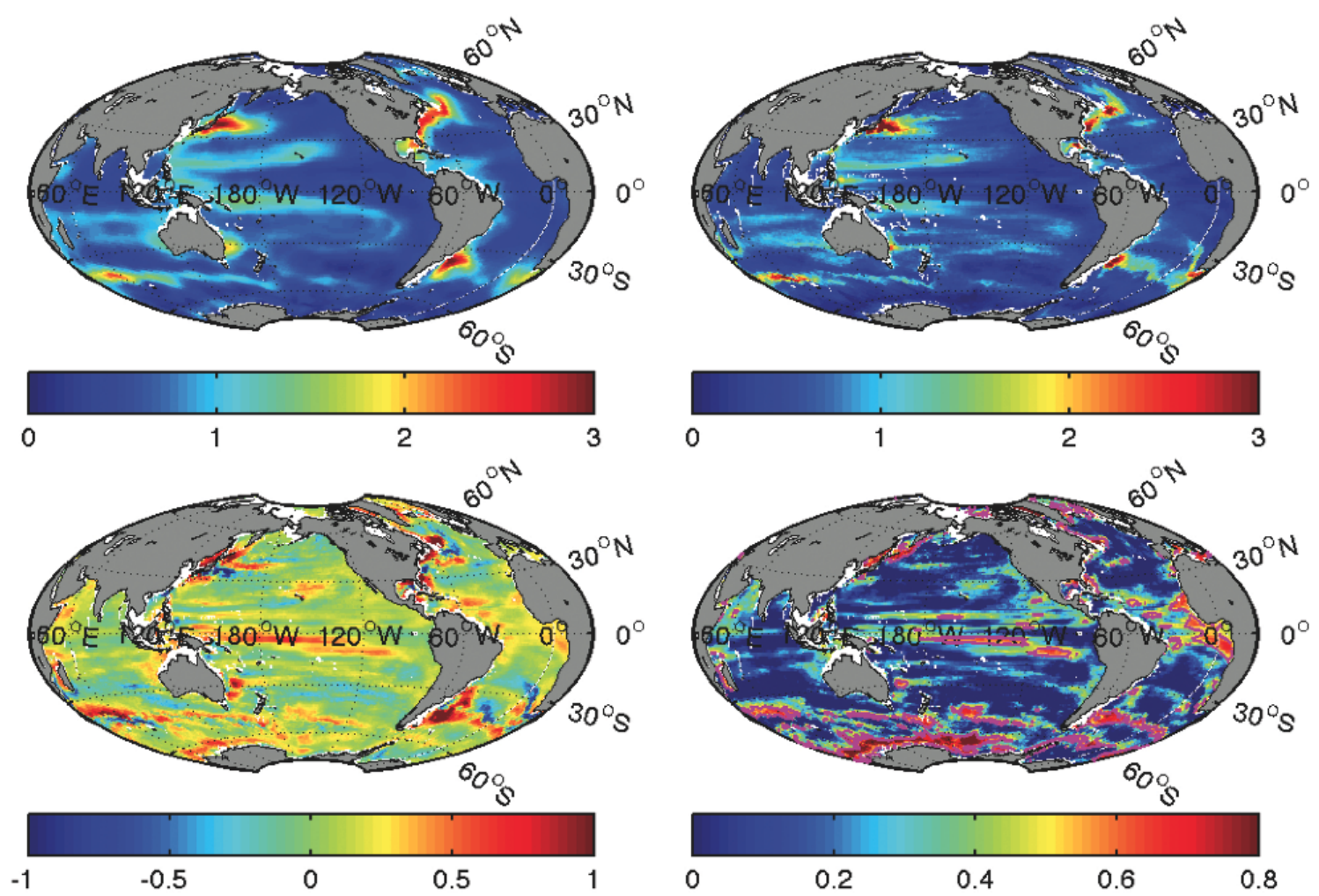

Figure 2-9: $\sigma_{T o}$ in ${ }^{\circ} C$ (upper left), $\sigma_{T m}$ in ${ }^{\circ} C$ (upper right), the misfit $\sigma_{T o}-\sigma_{T m}$ in ${ }^{\circ} \mathrm{C}$ (lower left) and the relative misfit $\left|\sigma_{T o}-\sigma_{T m}\right| / \sigma_{T o}$ (lower right) at $250 \mathrm{~m} . \sigma_{T o}$ $\left(\sigma_{T m}\right)$ is the standard deviation of observed (modeled) temperature omitting seasonal variability. Magenta contours in the lower right panel have the value of 0.5.

of latitude and depth. The relative misfit of temperature (salinity) variability is smaller than 0.5 in the upper $4000 \mathrm{~m}(2000 \mathrm{~m})$ in the mid- and low latitudes. The relative misfit of both the temperature and salinity variability is larger than 0.5 throughout the water column in the high latitudes. The relative misfit of salinity variability is generally larger than that of temperature variability below $2000 \mathrm{~m}$. Some representative cross sections of the hydrographic variability in the model are shown in Figure 2-11, as an example to illustrate the consistency between the observed and modeled hydrographic variability in the upper ocean. Similar to the corresponding observations (Figure 5 in Forget and Wunsch, 2007), the hydrographic variability in Figure 2-11 is large at the depth range with strong stratification in the equatorial 

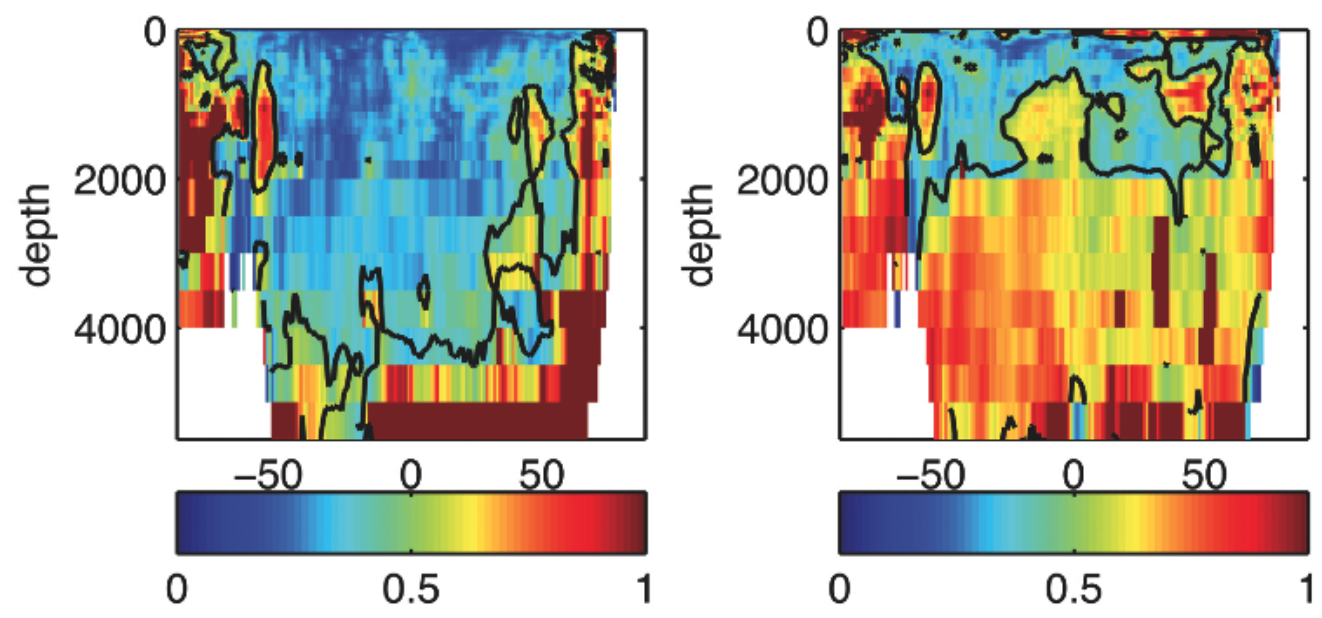

Figure 2-10: Zonally averaged relative misfit (left: $\left|\sigma_{T o}-\sigma_{T m}\right| / \sigma_{T o}$; right: $\left.\left|\sigma_{S o}-\sigma_{S m}\right| / \sigma_{S o}\right)$ as a function of latitude and depth $(m) . \quad \sigma_{S o}\left(\sigma_{S m}\right)$ is the standard deviation of observed (modeled) salinity omitting seasonal variability. Black contours have the value of 0.5 .

region and at the depth range with large horizontal temperature gradient.

To conclude, the variability in the model is overall consistent with the observed variability, especially in the upper ocean of mid- and low latitudes. The comparison of the modeled total kinetic energy with those from moorings also suggests that the ECCO2 model has better performance at low latitudes than high latitudes (Wortham, 2012). There are several possible reasons why the relative misfit is smaller in the upper ocean of these latitudes. First, the dominant scale of eddies is closely related to the first baroclinic deformation radius, which is smaller in high latitudes; thus the model resolution is not high enough to resolve motions on the deformation scale there. Second, the vertical resolution of the model is higher in the upper ocean than that in the deep ocean. Furthermore, the upper ocean is away from the topography, some of which is too steep or of too small scale for the model to resolve; thus the model performance in the deep ocean near topography may not be good. Third, fewer observations are available in the deep ocean and high latitudes, such as the Southern Ocean; thus the model solution is less constrained there. Fourth, the scarcity of 

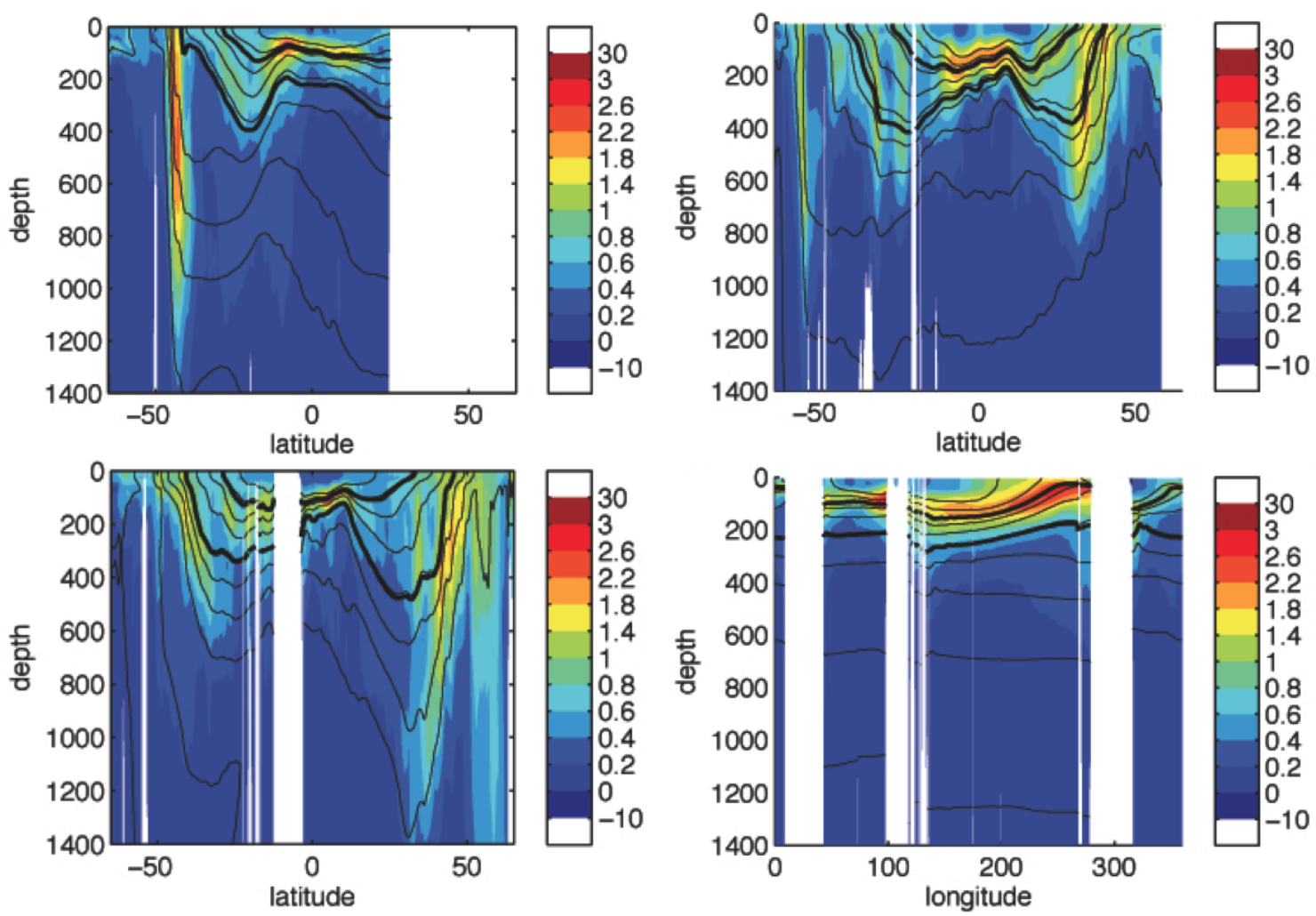

Figure 2-11: Cross sections of $\sigma_{T m}$ in ${ }^{\circ} \mathrm{C}$ in the Indian Ocean at $64.35^{\circ} \mathrm{E}$ (upper left), in the Pacific Ocean at $164.5^{\circ} \mathrm{E}$ (upper right), in the Atlantic Ocean at $322.5^{\circ} \mathrm{E}$ (lower left) and along the equator at $0.5^{\circ} \mathrm{S}$ (lower right). Black contours are isotherms and the thick ones denote the $14^{\circ} \mathrm{C}$ and $22^{\circ} \mathrm{C}$ isotherms. The depth is in $\mathrm{m}$.

observations in the deep ocean and high latitudes may contribute to the possible poor quality of the estimated hydrographic variability from Forget and Wunsch (2007) and thus contribute to the large relative misfit there. Similarly, the smaller relative misfit of temperature variability than that of salinity may also be partially due to the better quality of temperature observations.

\subsection{Conclusions}

The ECCO2 state estimate is used in our research presented in Chapters 3 and 4. This chapter briefly discusses the model setup, the state estimation method and the 
model-data misfit. It is an appropriate tool for the investigation of energy pathways and structures of oceanic eddies, and the diagnostic results in later chapters should be reasonable especially in the upper ocean of the mid- and low latitudes. The reasons are as follows: (1) The state estimation method is the Green function approach. The solution used in the thesis is essentially a free forward run using the optimized control parameters. Because no data is inserted in the forward run, neither unphysical jumps nor artificial sources/sinks are introduced in this dynamically consistent state estimate (Wunsch et al., 2009). Thus, the solution is useful for process studies and budget diagnosis (e.g. eddy energy budgets). (2) Due to the properly chosen subgrid parameterization schemes and the state estimation method, the properties of the model solution, such as the magnitude and the frequency-wavenumber spectrum of sea surface height anomaly, are realistic. (3) Also the model output is saved at a three-day interval, which is short enough to capture most eddy signals. (4) The horizontal resolution of the model (18 $\mathrm{km}$ on average) is high enough to simulate baroclinic/barotropic instability processes. The eddy energy level in the deep ocean is extremely low; thus, the low vertical resolution there probably will not affect much the estimate of the globally integrated energy conversion rates. (5) Several previous studies about eddies using the ECCO2 state estimate (e.g. Fu, 2009; Volkov et al., 2008; Volkov and $\mathrm{Fu}, 2008$ ) also indicate the utility of the solution. 


\section{Chapter 3}

\section{A description of eddy-mean flow interaction in the ECCO2 state estimate}

\subsection{Introduction}

The ocean circulation is generated as a result of the external forces including winds, tides and heat exchanges with the atmosphere (e.g. Ferrari and Wunsch, 2010; Huang, 2004). Several studies have described the spatio-temporal patterns of the wind work and have estimated that the total wind power input into the surface geostrophic flow in the global ocean is roughly $0.8 T W$ (e.g. Wunsch, 1998; Scott and Xu, 2009). However, the uncertainty of this number is significant (Zhai et al., 2012). The ways in which the energy, momentum, vorticity and enstrophy from these external forces move through the global ocean, transform in their nature and scale, are exchanged with the atmosphere and cryosphere, and are dissipated are extremely complicated. Many aspects of this process are still unknown and full descriptions about this do not 
exist.

The ocean circulation varies on a broad range of spatio-temporal scales, and the time-varying flows can exchange energy, vorticity and momentum with the time-mean circulation through eddy-mean flow interaction. These exchanges influence the nature of both the mean and the perturbations. Within the ocean, the time-mean circulation contains most of the potential energy, whereas the time-varying flow contains most of the kinetic energy. A large body of literature exists on the conversion of energy from the time-mean circulation to the time-varying flow through barotropic, baroclinic, mixed instability processes, etc. (e.g. Gill et al., 1974; Pedlosky, 1987; Vallis, 2006; Spall, 2000). Energy from the time-varying flow can also be transferred back to the time-mean circulation through a variety of processes including rectification and topography steering (e.g. Whitehead, 1975; McWilliams et al., 1978; Marshall, 1984; Johnson et al., 1992; Witter and Chelton, 1998); similar phenomena are found in atmospheric jet streams (e.g. Williams et al., 2007). At the same time, energy can also be redistributed among different spatial scales/vertical modes through energy cascades (e.g. Salmon, 1978; Scott and Wang, 2005; Fu and Flierl, 1980) and be transmitted over large distances through e.g. advection or the propagation of rings and waves (e.g. Flierl, 1977).

von Storch et al. (2012) studied the ocean Lorenz energy cycle using a $1 / 10^{\circ}$ global simulation and suggested that, even though the eddy-mean flow interaction in the ocean involves many physical processes, the dominant globally integrated energy pathway between eddies and the mean flow in both the ocean and the atmosphere is identical to the energy pathway in idealized local baroclinic instability processes (Pedlosky, 1987; Lorenz, 1955). The generation rate of eddy kinetic energy through this energy pathway in the global ocean is roughly one third of the total wind power input into the geostrophic flow (Ferrari and Wunsch, 2009; Scott and Xu, 2009). 
A large literature exists discussing the simple and yet compelling local baroclinic instability hypothesis, its plausibility in the mid-ocean, and its utility in explaining eddy properties and generation in the global ocean (e.g. Robinson and McWilliams, 1974; Held and Larichev, 1996; Venaille et al., 2011; Smith, 2007; Tulloch et al., 2011). This hypothesis has two aspects: (1) each region in the ocean is assumed to be horizontally homogeneous and thus all the energy released from the baroclinically unstable mean flow is used to sustain the local eddy energy growth, which is balanced by other terms in the eddy energy budget (e.g. mixing and dissipation); (2) the dominant source for eddy growth in this patch is the energy released from the mean flow through baroclinic instability, not from advection, external forcing, etc. (e.g. Tulloch et al., 2011). Observed eddies in the mid-ocean have similar properties to those from local linear baroclinic instability analysis and to those from relevant idealized experiments with reasonable parameters, indicating the plausibility of this hypothesis in the mid-ocean (Gill et al., 1974; Arbic and Flierl, 2004). Many oceanic problems, such as jet dynamics, eddy heat fluxes, time-dependent instabilities and energy cascades, have been investigated in the doubly-periodic two-layer model with vertical shear in which local baroclinic instability occurs (e.g. Panetta, 1993; Salmon, 1978; Thompson, 2010).

This study is concerned with the first aspect of the local baroclinic instability hypothesis, which is assumed in many instability theories (Pedlosky, 1987). The actual time-mean circulation is not homogeneous (Tulloch et al., 2011; Arbic, 2000), implying that the energy released from the mean flow through eddy-mean flow interaction can be transmitted to other regions through the divergence term (Section 3.2; Liang and Robinson, 2007; Kundu and Cohen, 2004). The amount of energy transmitted elsewhere and the impact of this nonlocal nature of eddy-mean flow interaction on energy cascades, eddy fluxes, jet dynamics and eddy properties are still 
largely unknown.

We do not have a theory to test. The goals of this mainly descriptive chapter are simply to (1) map the respective change rate of energy in eddies and the mean flow through eddy-mean flow interaction and (2) characterize the regional energy route through eddy-mean flow interaction and discuss whether the energy released from the mean flow is used to support the local eddy energy growth in energetic regions. The oceanic community lacks the long-term observations of global velocity, salinity and density fields, which are needed to pursue this study. However, previous studies show that diagnosing oceanic models is a useful way to explore oceanic energetics problems (e.g. Cox, 1987; von Storch et al., 2012; Zhai and Marshall, 2012). Here we use the ECCO2 state estimate, noting that it is dynamically consistent and thus applicable to the energy budget analysis, and with the implicit assumption that the simulated oceanic circulation is quantitatively accurate enough for the task.

Our results are not definitive and come with several caveats. First, the ECCO2 state estimate does not resolve submeoscale variability, and the fidelity of the mesoscale variability from the state estimate remains partially uncertain (Chapter 2). Second, an important assumption here is that the 16-year model output is long enough to separate the putative time-mean flow from the oceanic variability. Finally, the definition of available potential energy (APE) is arguable; and we assume that the definition based on the quasi-geostrophic assumption (Lorenz, 1955; Oort et al., 1989) is reasonable enough for the purpose here. These points are discussed to some extent later. More accurate or different answers might be obtained using other numerical models and other APE diagnostic frameworks. 


\subsection{Diagnostic framework}

\subsubsection{Derivation of the framework}

\section{On the notation}

Here we briefly discuss the notations used in this section. ${ }^{-}$denotes the time mean and ${ }^{\prime}$ denotes the deviation from the time mean. For example, we can split the zonal velocity $u$ and the in-situ density $\rho$ into the time-mean components and the deviation components, i.e.,

$$
\begin{aligned}
& u(x, y, z, t)=\bar{u}(x, y, z)+u^{\prime}(x, y, z, t), \\
& \rho(x, y, z, t)=\bar{\rho}(x, y, z)+\rho^{\prime}(x, y, z, t) .
\end{aligned}
$$

$<\cdot>$ denotes the global mean at a given depth. For example, the vertical profile of the global- and time-mean in-situ density is

$$
<\overline{\rho(x, y, z, t)}>=<\bar{\rho}(x, y, z)>=\tilde{\rho}(z)
$$

Thus, we can split the in-situ density into two components, i.e.,

$$
\rho(x, y, z, t)=\rho^{*}(x, y, z, t)+\tilde{\rho}(z) .
$$

We can split the hydrostatic pressure $p$ in a similar way, i.e.,

$$
p(x, y, z, t)=p^{*}(x, y, z, t)+\tilde{p}(z) .
$$

Note that

$$
\rho^{*^{\prime}}(x, y, z, t)=\rho^{*}(x, y, z, t)-\overline{\rho^{*}(x, y, z, t)}=\rho^{\prime}(x, y, z, t),
$$




$$
p^{*^{\prime}}(x, y, z, t)=p^{*}(x, y, z, t)-\overline{p^{*}(x, y, z, t)}=p^{\prime}(x, y, z, t)
$$

\section{Definition of kinetic energy and available potential energy}

Ocean variability encompasses a continuum of spatial scales, ranging from submesocale and mesoscale motions to gyre shifts and basin oscillations; it also spans a wide range of temporal scales, ranging from superintertial to seasonal and decadal variability. In this chapter, mean flow refers to the flow temporally averaged over the specific 16 years available from the ECCO2 model. The entire time-varying flow in the ECCO2 model, which is the deviation from the 16-year average and independent of spatial scale, is termed "eddies" as a short hand. One caveat is that decadal variability and submesoscale variability at a horizontal scale of a few kilometers, though not resolved in the ECCO2 model, might contribute significantly to the energy budget. Also note that eddies at different spatio-temporal scales probably contribute differently to the eddy-mean flow interaction, though we do not consider this issue here.

Thus, the kinetic energy in the mean flow is defined as

$$
e_{\text {mean }}^{k}(x, y, z)=\frac{1}{2} \rho_{0}\left(\bar{u}^{2}+\bar{v}^{2}\right)
$$

and the kinetic energy in the time-varying flow is defined as

$$
e_{e d d y}^{k}(x, y, z)=\frac{1}{2} \rho_{0} \overline{\left(u^{\prime 2}+v^{\prime 2}\right)}
$$

where $\rho_{0}$ is the constant reference density $\left(1027.5 \mathrm{~kg} / \mathrm{m}^{3}\right.$ in the ECCO2 state estimate). Here vertical velocities have negligible contribution to kinetic energy and thus are being ignored.

The concept of available potential energy (APE), introduced by Margules (1905), 
has been widely used to explore the energy cycle in the atmosphere and the ocean (e.g. Lorenz, 1955; Oort et al., 1989; Huang, 2005). It refers to the difference in potential energy between the actual state, which is the state that we are studying (e.g. oceanic state in the ECCO2 state estimate), and the reference state, which has the minimum amount of potential energy (Huang, 2005). APE in a Boussinesq ocean is

$$
\prod=g \overline{\int_{v}\left(\rho z-\rho_{r} Z\right) d V}
$$

where $v$ denotes some volume, $\rho_{r}(\rho)$ and $Z(z)$ are the in-situ density and vertical position of water parcels in the reference (actual) state (e.g. Winters et al., 1995). The reference state can be determined through adiabatic mass adjustment using an iterative algorithm and then APE can be determined (Huang, 1998). However, the partition of APE in Eq. 3.10 into eddy available potential energy (EAPE) and mean available potential energy (MAPE) is still an open question, as it is challenging to quantify the contribution of eddies to $Z$ (Hughes et al., 2009). Developing an eddymean flow interaction energetics framework based on this definition is a separate problem, which we will not address here.

Instead, this study uses the quasi-geostrophic definition of APE, which has been widely used in the ocean studies (e.g. Oort et al., 1989, 1994; Pedlosky, 1987; Huang, 2010; von Storch et al., 2012; Brown and Fedorov, 2010):

$$
\chi(x, y, z)=-\frac{g}{2 n_{0}} \overline{[\rho(x, y, z, t)-<\overline{\rho(x, y, z, t)}>]^{2}}=-\frac{g}{2 n_{0}} \overline{\rho^{*}(x, y, z, t)^{2}},
$$

where $\rho(x, y, z, t)$ is the in-situ density at the actual state. The time mean and global mean of $\rho(x, y, z, t)(<\overline{\rho(x, y, z, t)}\rangle)$ is the in-situ density at the reference state. $n_{0}$ 
is the time mean and global mean of the vertical gradient of local potential density

$$
n_{0}(z)=-\frac{\rho_{0}}{g} N^{2}(z)=<\overline{\left[\frac{\partial \rho(x, y, z, t)}{\partial z}\right]_{x, y, t}}>-<\overline{\left[\frac{\partial \rho(S, \theta, z)}{\partial z}\right]_{S, \theta}}>
$$

where $N^{2}(z)$ is the time mean and global mean of Brunt-Väisälä frequency (Huang, 2010), $S$ and $\theta$ denote salinity and potential temperature and they are functions of space and time. Note that if the goal is to evaluate the APE budget of mesoscale motions, one should choose spatial average over a few degrees, not the global average as the reference state (Huang, 2010). Here our focus is on exploring the interaction between the mean flow and the time-varying flow, which has a wide range of spatial scales. Thus, it is reasonable to choose the global mean as the reference state. This choice of the reference state has recently been used to evaluate the Lorenz energy cycle in the ocean and the energy budget of time-varying flows with the period of 10 minutes $\sim 10$ years from a global perspective (von Storch et al., 2012). Our diagnostic framework introduced next (Eq. $3.15 \sim$ Eq. 3.18) is mathematically self-consistent and is useful to evaluate the eddy-mean flow interaction problem.

Here we provide our definition of MAPE and EAPE. The total available potential energy (Eq. 3.11) is the difference between the potential energy stored in the snapshot isopycnals in the actual state and that in the reference state. Since density in the actual state is the instantaneous density $\rho(x, y, z, t)$, the density in the time-mean actual state is $\overline{\rho(x, y, z, t)}$. MAPE is the difference between the potential energy stored in the time-mean actual state and that in the reference state:

$$
\chi_{\text {mean }}(x, y, z)=-\frac{g}{2 n_{0}} \overline{\rho^{*}}(x, y, z)^{2}
$$

EAPE is the difference between the potential energy in the actual state and that in 
the time-mean actual state:

$$
\chi_{e d d y}(x, y, z)=-\frac{g}{2 n_{0}} \overline{\rho^{*^{\prime}}(x, y, z, t)^{2}}=-\frac{g}{2 n_{0}} \overline{\rho^{\prime}(x, y, z, t)^{2}} .
$$

Note that $\chi(x, y, z)=\chi_{\text {mean }}(x, y, z)+\chi_{\text {eddy }}(x, y, z)$.

\section{Energy equations for the mean flow and eddies}

In some atmospheric and Southern Ocean studies, mean flow is often defined as the zonally averaged flow, and the transformed Eulerian mean framework is used to explore eddy-mean flow interaction (e.g. Vallis, 2006; Plumb and Ferrari, 2005; Kuo et al., 2005). Here we need a framework consistent with our definition of eddies and mean flow. A detailed derivation of the kinetic and available potential energy equations appropriate to the ECCO2 state estimate is provided in the appendix (Sections 3.6.1 and 3.6.2). These equations are

$$
\begin{aligned}
& \frac{\partial}{\partial t} e_{\text {mean }}^{k}+\nabla \cdot\left(\overline{\mathbf{u}} e_{\text {mean }}^{k}\right)+\nabla \cdot\left(\overline{\mathbf{u}} \overline{p^{*}}\right)=-B_{M K E}+C_{M K E}+D_{M K E} \\
& \left.\left.\frac{\partial}{\partial t} e_{e d d y}^{k}+\nabla \cdot \overline{\left[\mathbf{u} \frac{1}{2} \rho_{0}\left(u^{\prime 2}+v^{\prime 2}\right)\right.}\right]+\nabla \cdot \overline{\left(\mathbf{u}^{\prime} p^{\prime}\right.}\right)=B_{E K E}+C_{E K E}+D_{E K E} \\
& \frac{\partial}{\partial t} \chi_{\text {mean }}+\nabla \cdot\left(\overline{\mathbf{u}} \chi_{\text {mean }}\right)=B_{M A P E}+B_{M K E}+D_{M A P E}+R_{\chi_{\text {mean }}} \\
& \frac{\partial}{\partial t} \chi_{e d d y}+\nabla \cdot \overline{\left[-\mathbf{u} g \rho^{\prime 2} /\left(2 n_{0}\right)\right]}=B_{E A P E}-B_{E K E}+D_{E A P E}+R_{\chi_{e d d y}},
\end{aligned}
$$

where

$$
\nabla=\frac{\partial}{\partial x} \widehat{i}+\frac{\partial}{\partial y} \widehat{j}+\frac{\partial}{\partial z} \widehat{k}, \frac{\partial}{\partial z} p^{*}=-\rho^{*} g, \frac{\partial}{\partial z} p^{\prime}=-\rho^{\prime} g
$$

and $\mathbf{u}$ is the three-dimensional velocity vector.

The terms on the left-hand side of Eqs. 3.15-3.18 represent the local change rates of the energy and redistribution of energy through advection and pressure work. The $B$ terms, listed in Table 3.1, are eddy-mean flow interaction terms related to the eddy 
density flux. The $C$ terms, listed in Table 3.1, are eddy-mean flow interaction terms related to eddy momentum flux. The $D$ terms denote the change rate of MAPE and EAPE due to vertical mixing, heat and freshwater fluxes $\left(D_{M A P E}\right.$ and $\left.D_{E A P E}\right)$ and the change rate of kinetic energy in the mean flow (MKE) and kinetic energy in eddies (EKE) due to friction, wind stress and bottom drag $\left(D_{M K E}\right.$ and $\left.D_{E K E}\right)$. The $R$ terms and the vertical advection of APE are additional terms with higher order Rossby numbers, which do not exist in the quasi-geostrophic framework. These terms can be neglected below the surface mixed layers and away from convective regions and are ignored in our later discussion. Note that the local change rates (the first term on the left-hand side) in Eqs. 3.15-3.18 are negligible.

To tackle the proposed questions, we focus on determining the eddy-mean flow interaction terms listed in Table 3.1. Evaluating all the terms of these energy equations, even with a model, is formidable undertaking for several reasons: certain variables, such as temporally/spatially varying viscosity and diffusivity, are not available; we use three-day averaged fields and thus cannot determine the contribution of highfrequency motions to the energy budget.

Table 3.1: The eddy-mean flow interaction terms diagnosed in this study. $\mathbf{u}_{\mathbf{H}}$ is horizontal velocity vector and $\nabla_{H}=\frac{\partial}{\partial x} \widehat{i}+\frac{\partial}{\partial y} \widehat{j} . \quad v$ is meridional velocity and $w$ is vertical velocity.

\begin{tabular}{lll}
\hline \hline term & mathematical form & meaning \\
\hline$B_{M A P E}$ & $\frac{g}{n_{0}} \overline{\rho^{*}} \nabla_{H} \cdot \overline{\left(\overline{\mathbf{u}_{\mathbf{H}}^{\prime} \rho^{\prime}}\right)}$ & MAPE change rate due to horizontal density \\
& flux \\
$B_{E A P E}$ & $\frac{g}{n_{0}} \overline{\mathbf{u}_{\mathbf{H}}^{\prime} \rho^{\prime} \cdot \nabla_{H} \overline{\rho^{*}}}$ & $\begin{array}{l}\text { EAPE+EKE change rate due to horizontal den- } \\
\text { sity flux }\end{array}$ \\
$B_{E K E}$ & $-g \overline{\rho^{\prime} w^{\prime}}$ & Gain rate of EKE from EAPE \\
$C_{M K E}$ & $-\rho_{0}\left[\bar{u} \nabla \cdot\left(\overline{u^{\prime} \mathbf{u}^{\prime}}\right)+\bar{v} \nabla \cdot\left(\overline{v^{\prime} \mathbf{u}^{\prime}}\right)\right]$ & MKE change rate due to eddy momentum flux \\
$C_{E K E}$ & $-\rho_{0}\left(\overline{u^{\prime} \mathbf{u}^{\prime}} \cdot \nabla \bar{u}+\overline{v^{\prime} \mathbf{u}^{\prime}} \cdot \nabla \bar{v}\right)$ & EKE change rate due to eddy momentum flux \\
\hline
\end{tabular}




\subsubsection{Interpretation}

\section{Local vs. nonlocal eddy-mean flow interaction}

Here we provide our definitions of local eddy-mean flow interaction and non-local eddy-mean flow interaction. The rate of energy released from the mean flow through eddy-mean flow interaction is the sum of $B_{M A P E}$ and $C_{M K E}$. We can gain some insight into the pathways of energy released from the mean flow through the equation for total mechanical energy:

$$
\frac{\partial}{\partial t}\left(e_{\text {mean }}^{k}+e_{e d d y}^{k}+\chi_{\text {mean }}+\chi_{e d d y}\right)=\left(B_{M A P E}+B_{E A P E}+C_{M K E}+C_{E K E}\right)+R e s,
$$

which is the sum of Eq. $3.15 \sim$ Eq. 3.18. The $B$ and $C$ terms on the right hand of Eq. 3.19 are the change rates of total kinetic and available potential energy at a given location due to eddy-mean flow interaction. Res represents all the other terms. If the sum of the $B$ and $C$ terms in Eq. 3.19 integrated over a region is negligible, all the energy released from the mean flow is converted to eddy energy in the same region and thus the eddy-mean flow interaction in this case is local. On the contrary, if it is not negligible, some of the energy released from the mean flow is not used to sustain the eddy growth in the same region and the eddy-mean flow interaction is nonlocal. This is further illustrated in Figure 3-1. Note that other nonlocal terms (advection and the work done by pressure work) exist in all the oceanic regions; however, the definition of local vs. nonlocal eddy-mean state interaction in this study is based on the sum of the $B$ and $C$ terms, not based on the magnitude of advection term, pressure work, etc. Thus, though the energy gained by eddies is always balanced by other terms in the eddy energy budgets including advection and pressure work, the eddy-mean flow interaction process is termed local in this study, as long as the sum of the $B$ and $C$ terms in Eq. 3.19 integrated over a region is negligible. 


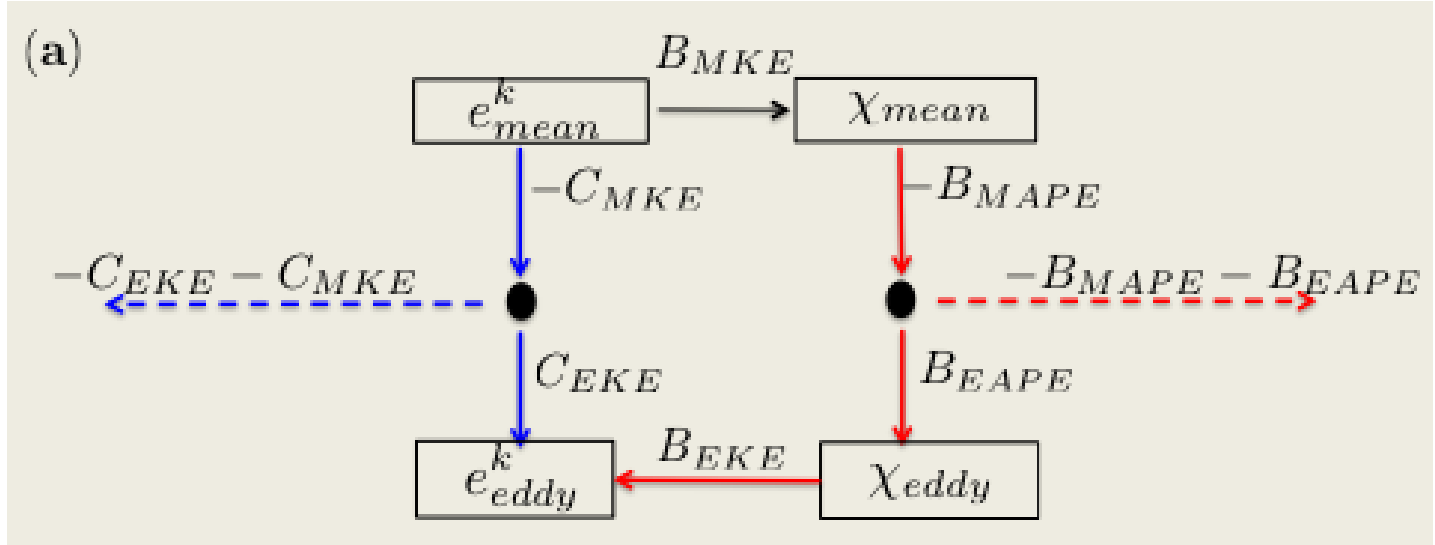

(b)

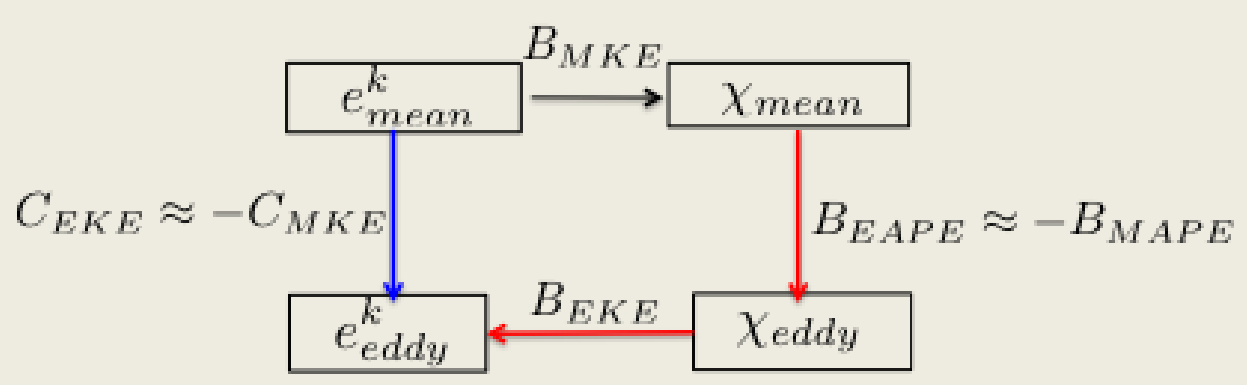

Figure 3-1: Schematics illustrating the energy transfer through eddy-mean flow interaction (other energy terms are omitted, except $B_{M K E}$ ). (a) illustrates the case when eddy-mean flow interaction is nonlocal. Only part of the energy released from the unstable mean flow through terms $C_{M K E}$ and $B_{M A P E}$ is used to support the local eddy growth; the rest of the energy released is transported elsewhere through the divergence terms $C_{E K E}+C_{M K E}$ and $B_{M A P E}+B_{E A P E}$. (b) illustrates the case when the eddy-mean flow interaction is local: in this case, the divergence terms are approximately zero and thus all the energy released from the unstable mean flow through eddy-mean flow interaction transfers to eddies in the same region.

The nonlocal interaction can occur because the energy release rate from the mean flow due to eddy-mean flow interaction in a region $\left(B_{M A P E}\right.$ and $\left.C_{M K E}\right)$ is not necessarily equal to the gain rate of energy in the eddies $\left(B_{E A P E}\right.$ and $\left.C_{E K E}\right)$ in the same region:

$$
\begin{gathered}
B_{M A P E}+B_{E A P E}=\nabla_{H} \cdot\left(\overline{\mathbf{u}_{\mathbf{H}}^{\prime} \rho^{\prime}} \frac{g}{n_{0}} \overline{\rho^{*}}\right) \neq 0 \\
C_{M K E}+C_{E K E}=-\rho_{0}\left[\nabla \cdot \overline{\left(\bar{u} u^{\prime} \mathbf{u}^{\prime}\right)}+\nabla \cdot \overline{\left(\bar{v} v^{\prime} \mathbf{u}^{\prime}\right)}\right] \neq 0 .
\end{gathered}
$$


These terms in Eqs. 3.20 and 3.21 have a divergence form and thus the global integral is zero ${ }^{1}$. Therefore, the part of energy released from the mean flow which is not used to sustain the local eddy energy growth is essentially transported elsewhere through the divergence terms (Eqs. 3.20 and 3.21), as illustrated in Figure 3-1.

Diagram (a) in Figure 3-1 is different from the traditional Lorenz energy diagram (e.g. Lorenz, 1955; von Storch et al., 2012) by including the divergence components $\left(B_{M A P E}+B_{E A P E}, C_{E K E}+C_{M K E}\right)$. The global integral of the divergence components is zero and thus it is not included in the traditional Lorenz energy diagram, which is used to illustrate the energy pathway in the global atmosphere or ocean. Diagram (a) can be used to illustrate the energy route for eddy-mean flow interaction in selected regions. Diagram (b) in Figure 3-1 illustrates the local eddy-mean flow interaction route.

\section{Divergent eddy fluxes and pathway of energy gained by eddies}

Using the concepts of divergent eddy fluxes and rotational eddy fluxes, Marshall and Shutts (1981) proposed a pathway of $B_{E A P E}$, which is the gain rate of eddy energy by eddy-mean flow interaction through horizontal eddy density fluxes. Eddy density fluxes can be decomposed into two parts:

$$
\overline{\mathbf{u}_{\mathbf{H}}^{\prime} \rho^{\prime}}={\overline{\left(\mathbf{u}_{\mathbf{H}}^{\prime} \rho^{\prime}\right)_{D}}}+{\overline{\left(\mathbf{u}_{\mathbf{H}}^{\prime} \rho^{\prime}\right)_{R}}}
$$

where ${\overline{\left(\mathbf{u}_{\mathbf{H}}^{\prime} \rho^{\prime}\right)}}_{D}$ is the divergent eddy flux satisfying

$$
\nabla_{H} \times{\overline{\left(\mathbf{u}_{\mathbf{H}}^{\prime} \rho^{\prime}\right)}}_{D}=0
$$

and ${\overline{\left(\mathbf{u}_{\mathbf{H}}^{\prime} \rho^{\prime}\right)}}_{R}$ is the rotational eddy flux (e.g. Fox-Kemper et al., 2003). Thus, we can

\footnotetext{
${ }^{1}$ The global integral of $B_{M A P E}+B_{E A P E}$ is only approximately zero, as we ignored the vertical eddy density flux contribution (see Section 3.6.2).
} 
split $B_{E A P E}$ into two parts:

$$
B_{E A P E}=B_{E A P E, D}+B_{E A P E, R}
$$

where

$$
B_{E A P E, D}=\frac{g}{n_{0}} \overline{\left(\overline{\mathbf{u}_{\mathbf{H}}^{\prime} \rho^{\prime}}\right)_{D}} \cdot \nabla_{H} \overline{\rho^{*}}, B_{E A P E, R}=\frac{g}{n_{0}}\left(\overline{\mathbf{u}_{\mathbf{H}}^{\prime} \rho^{\prime}}\right)_{R} \cdot \nabla_{H} \overline{\rho^{*}}
$$

Marshall and Shutts (1981) investigated the pathway of $B_{E A P E}$ in a steady quasigeostrophic system satisfying the following criteria: the sources/sinks of EAPE and the advection of EAPE by eddies are negligible in the EAPE budget, the mean flow roughly aligns with the mean density contours and the mean stream function is linearly related to the mean density. They proved that, in such an idealized system, $B_{E A P E, D}$ balances $B_{E K E}$ and $B_{E A P E, R}$ balances the advection of EAPE by the mean flow, i.e.,

$$
B_{E A P E, D}=B_{E K E}, \text { and } B_{E A P E, R}=\overline{\mathbf{u}_{\mathbf{H}}} \cdot \chi_{e d d y} .
$$

They further infer that, in more general cases, $B_{E A P E, R}$ is probably balanced by the advection of EAPE and thus does not contribute to the energy transfer from EAPE to EKE.

We discuss the applicability of the above general statement in some regions in Section 3.4.2. Specifically, we infer the magnitude of $B_{E A P E, R}$ based on some previous literature and compare it with the magnitude of the horizontal advection term in the EAPE budget in the ECCO2 state estimate. In practice, the decomposition of eddy density fluxes into divergent and rotational components is sensitive to the boundary condition imposed in a bounded domain (e.g. Eden et al., 2007; Fox-Kemper et al., 2003). Thus, the diagnosis of $B_{E A P E, R}$ in the ECCO2 state estimate is left for future work. 
We can see, from Table 3.1, that only the divergent component of eddy density fluxes contributes to the energy release from the mean state through eddy density fluxes $\left(B_{M A P E}\right)$, as

$$
\left.\nabla_{H} \cdot\left(\overline{\mathbf{u}_{\mathbf{H}}^{\prime} \rho^{\prime}}\right)=\nabla_{H} \cdot \overline{\left(\mathbf{u}_{\mathbf{H}}^{\prime} \rho^{\prime}\right.}\right)_{D} .
$$

Thus, the divergent component of eddy fluxes is more relevant to eddy parameterization problems (e.g. Griesel et al., 2009). However, both the divergent and rotational components of eddy fluxes contribute to the divergence terms $\left(B_{M A P E}+B_{E A P E}\right.$ and $\left.C_{M K E}+C_{E K E}\right)$.

\subsection{ECCO2 state estimate evaluation}

The model configuration and model-data comparison about eddies are discussed in Chapter 2. This section further discusses the fidelity of the 16-year ECCO2 state estimate in the aspect of energetics and eddy-mean flow interaction.

\subsubsection{On the length of the record}

One question is whether the 16-year record available from the ECCO2 state estimate is long enough to evaluate the eddy-mean flow interaction terms (the $B$ and $C$ terms). These terms involve eddy momentum and eddy density fluxes. Are the amplitude and spatial pattern of eddy momentum and density fluxes sensitive to the record length?

Previous studies and our analysis show that large-scale features in the time-mean eddy fluxes are not very sensitive to the record length. The characteristics of surface momentum fluxes at the Kuroshio and Gulf Stream Extension regions estimated using a 5-year altimetric data are similar to those estimated from the 13-year data (Greatbatch et al., 2010). Our analysis also shows that the spatial pattern and mag- 
nitude of eddy fluxes from the 16-year record is remarkably similar to those estimated from shorter records. For example, $B_{E K E}$ (the time-mean vertical eddy density flux multiplied by $-g$ ) estimated from the 1992-1997 output and that from the 1992-2007 output in the global ocean have a spatial correlation of 0.8 . The ratio of the globally integrated $B_{E K E}$ based on the 16-year record and that based on the 6-year record is one. Figure 3-2 shows the comparison in the Kuroshio Extension region. The magnitude of the two patterns is almost the same. The large-scale features survive even in the estimates using the 6-year record: $B_{E K E}$ is positive (negative) in the western (eastern) part of the extension regions. This suggests that the large-scale features and globally integrated values are not very sensitive to the record length. Thus, the 16-year record is probably long enough for us to characterize the large-scale features of the global energetics patterns and the energy route averaged in selected regions.
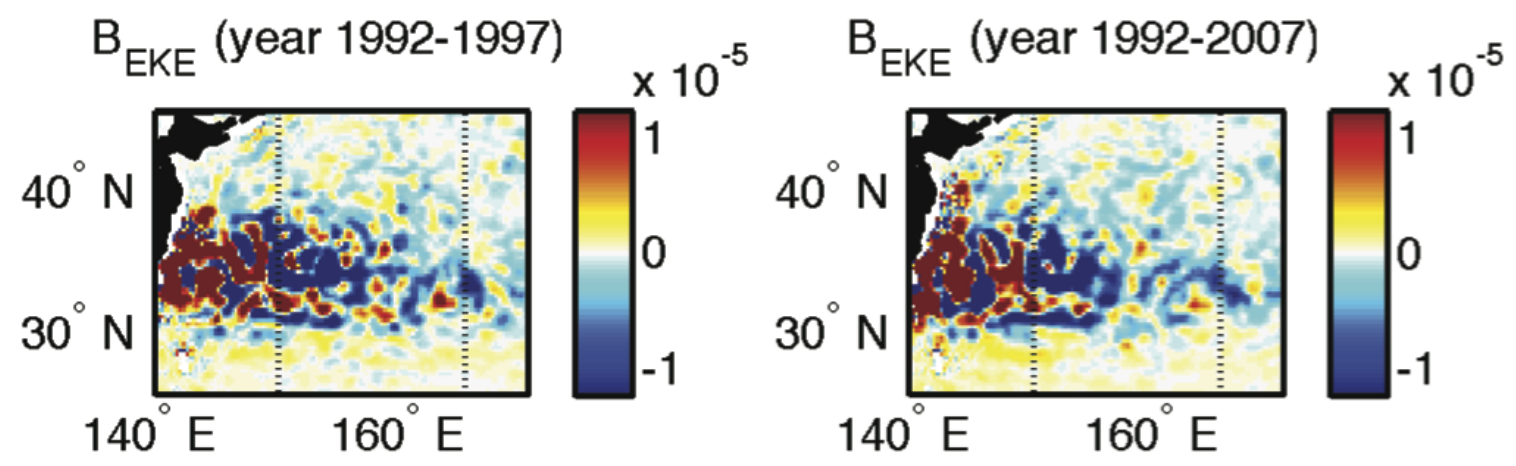

Figure 3-2: $B_{E K E}$ in $W / \mathrm{m}^{3}$ at $550 \mathrm{~m}$ in the Kuroshio Extension region, estimated from the 6-year and 16-year ECCO2 state estimate.

Small-scale features still exist even in our estimate based on the 16-year record (Figure 3-2). Where do the small-scale features in the time-mean eddy fluxes come from? Oceanic motions and the associated hydrographic field have a wide-range of spatial scales at each frequency (Wortham, 2012), as a result of eddy-eddy interaction, instability, and the wide-range of spatial scales in external forcing, topography, coastlines, etc. Consequently, small-scale features should exist in the time-mean eddy 
fluxes. The amplitude, position and structures of these small-scale features will probably change as the record length increases. However, the detailed description and understanding of these small-scale features are left for future work, as our focus here is on the large-scale patterns and regional characteristics.

\subsubsection{Model-data comparison of energetics}

\section{On the spatial pattern}

Chapter 2 compares the magnitude and spatial patterns of the oceanic variability in the ECCO2 state estimate with the observed variability in Forget and Wunsch (2007). To determine whether the ECCO2 state estimate is reasonable to evaluate the proposed problem, we also need to assess whether the spatial patterns of eddy-mean flow interaction terms in the model are consistent with observations. However, testing the model fidelity in this aspect is challenging and a research topic by itself, mostly due to the lack of long-term and large-area salinity, density and velocity observations.

Using the altimetric data, we can evaluate the time-mean contribution of geostrophic flows to $C_{E K E}$ at the oceanic surface in the ECCO2 model, that is,

$$
C_{E K E, g e o}=-\rho_{0}\left(\overline{u_{\text {geo }}^{\prime} \vec{u}_{\text {geo }}^{\prime}} \cdot \nabla_{H} \bar{u}_{\text {geo }}+\overline{v_{\text {geo }}^{\prime} \vec{u}_{\text {geo }}^{\prime}} \cdot \nabla_{H} \bar{v}_{\text {geo }}\right) .
$$

$u_{\text {geo }}\left(v_{\text {geo }}\right)$ is the zonal (meridional) geostrophic velocity estimated from sea surface height:

$$
v_{g e o}=\frac{g}{f} \frac{\partial \eta}{\partial x} \text { and } u_{g e o}=-\frac{g}{f} \frac{\partial \eta}{\partial y},
$$

with $f$ is the Coriolis parameter and $\eta$ the sea surface height. Note that the concept of $C_{E K E \text {,geo }}$ breaks down at the equator due to the vanishing of $f$ there; thus the equatorial regions are masked in the global pattern of $C_{E K E \text {,geo }}$ shown in Figure 3-3. 
The spatial pattern of $C_{E K E, g e o}$ estimated from the ECCO2 state estimate is similar to that from the altimetry in the off-equatorial regions (Figure 3-3). Both maps show large magnitudes of $C_{E K E, g e o}$ in the western boundary currents and the Southern Ocean. Large magnitudes of $C_{E K E, g e o}$ in the Southern Ocean occur within roughly the same longitude ranges in these two maps. The zonally integrated values from the ECCO2 state estimate and altimetry are also very similar in the off-equatorial regions: both with peaks at $40^{\circ} \mathrm{S}, 25^{\circ} \mathrm{N}$ and $35^{\circ} \mathrm{N}$ (not shown). However, the ECCO2 model underestimates $C_{E K E \text {,geo }}$ in the equatorial region and exploring the reasons is beyond the scope of discussion here.

Another similarity is that, in the Southern Ocean and western boundary extension regions, both positive and negative spots exist, though their detailed locations are only roughly the same in the two maps. We also computed $C_{E K E \text {,geo }}$ using the 8-year altimetry and ECCO2 state estimate (1993-2000). The location of these positive/negative spots based on the short record are the same as those based on the long record in roughly $80 \%$ of the global ocean. The short-record and the long-record estimated time-mean fields based on the ECCO2 model have a correlation of 0.9. The short-record and the long-record estimated fields based on the altimetry have a correlation of 1.0.

\section{On the globally integrated values}

Another extensively explored topic related to eddy-mean flow interaction is the kinetic energy budget. Table 3.2 compares the globally integrated values from the ECCO2 state estimate with those in previous studies based on observations, models and parameterization schemes. The global integrals of $B_{E K E}$ and the wind power input into the surface geostrophic flow $\left(\tau_{s} \cdot \mathbf{u}_{\text {geo }}\right)$ are consistent with previous estimates.

The work done by the fluctuating winds $\left(\tau_{s}^{\prime} \cdot \mathbf{u}_{g e o}^{\prime}\right)$ appears overestimated in the ECCO2 model. 


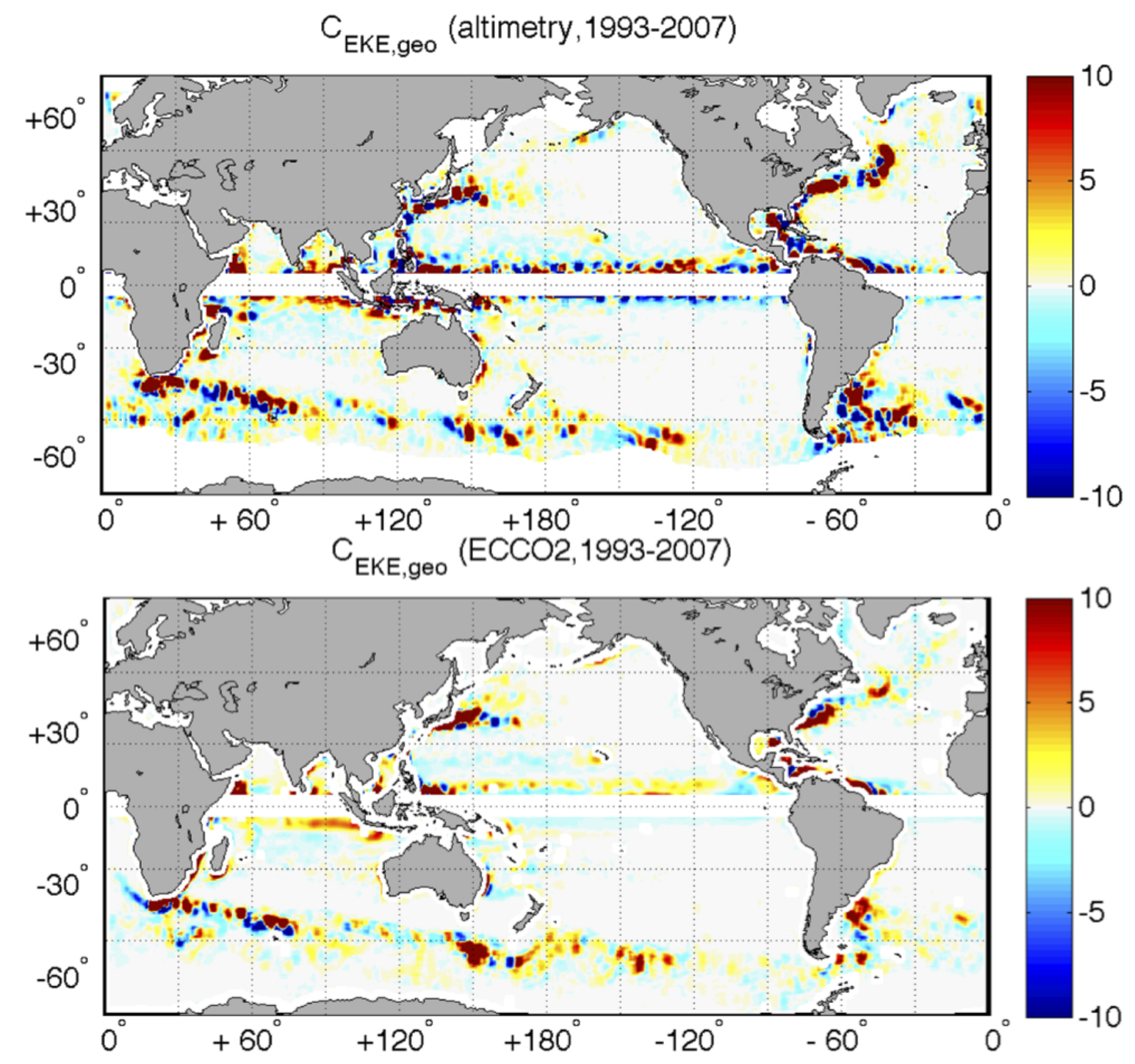

Figure 3-3: The smoothed $C_{E K E, g e o}$ in $\mu \mathrm{W} / \mathrm{m}^{3}$ at the oceanic surface using the weekly sea surface height during 1993-2007 from the altimetry (upper) and from the ECCO2 state estimate (lower). The regions within 3 degree of the equator are masked. The smoother is 3-degree running average. Both patterns are dominated by large values in the western boundary currents and the Southern Ocean and both are patchy in the Southern Ocean.

The bottom drag dissipation $\left(\tau_{b} \cdot \mathbf{u}_{b}\right)$ in the ECCO2 model may be underestimated to some extent (Table 3.2). Consistently, Wortham (2012) found that the total kinetic energy in the ECCO2 state estimate below $2000 m$ at the mooring sites is approximately half of the kinetic energy observed from the current meters. On the other 
Table 3.2: The 16-year average of the globally integrated energy terms from the ECCO2 state estimate and previous estimates $(T W)$. The errors shown are $\sigma / \sqrt{N}$, where $\sigma$ is the standard deviation and $\mathrm{N}$ is the number of degrees of freedom (Section 3.6.3). The global integral of the wind power input excludes the equatorial region (within $\pm 3^{\circ}$ of the equator), but the the global integrals of other terms listed include the equatorial region. $\tau_{s}$ is wind stress, $\mathbf{u}_{g e o}$ is the surface geostrophic velocity, $\tau_{b}$ is bottom drag based on the quadratic drag law, and $\mathbf{u}_{b}$ is bottom velocity.

\begin{tabular}{|c|c|c|}
\hline Energy terms & Estimates from ECCO2 & Previous estimates \\
\hline$B_{E K E}$ & $0.31 \pm 0.01$ & $\begin{array}{l}(0.2 \sim 0.8) \text { from Wunsch and Ferrari } \\
(2004) ; 0.3 \text { from Ferrari and Wunsch } \\
(2009) .\end{array}$ \\
\hline$\tau_{s} \cdot \mathbf{u}_{g e o}$ & $0.81 \pm 0.02$ & $\begin{array}{l}0.88 \text { from Wunsch }(1998) ; 0.75 \sim 0.9 \text { from } \\
\text { Scott and Xu }(2009) .\end{array}$ \\
\hline$\tau_{s}^{\prime} \cdot \mathbf{u}_{g e o}^{\prime}$ & $0.12 \pm 0.00$ & $\begin{array}{l}(0.04 \sim 0.06) \text { from Zhai et al. (2012); } 0.04 \\
\text { from Wunsch }(1998) .\end{array}$ \\
\hline$\tau_{b} \cdot \mathbf{u}_{b}$ & $0.03 \pm 0.00$ & $\begin{array}{l}\text { at least } 0.2 \text { from Sen et al. }(2008) ;(0.14 \sim \\
0.65) \text { from Arbic et al. }(2009) .\end{array}$ \\
\hline
\end{tabular}

hand, differences in estimation methods probably also contribute to the difference between our estimates and previous estimates about bottom drag dissipation. Sen et al. (2008) estimated the bottom drag dissipation using mooring observations, which are very sparse in space. Arbic et al. (2009) estimated the bottom drag dissipation from the snapshot bottom velocity in oceanic models, which includes the high frequency component. However, our estimate is calculated from the 3-day averaged bottom velocity.

Besides bottom drag, the kinetic energy in the model can also be dissipated through interior vertical viscosity and biharmonic viscosity. From the momentum equations in the $\mathrm{x}$ and $\mathrm{y}$ direction in the appendix (Section 3.6.1), we can get the formula of the dissipation in the global ocean interior due to vertical viscosity $A_{z}$ :

$$
\epsilon=\rho_{0} \iiint A_{z}(x, y, z, t)\left[\left(\frac{\partial u}{\partial z}\right)^{2}+\left(\frac{\partial v}{\partial z}\right)^{2}\right] d x d y d z .
$$

The output for the temporally and spatially varying $A_{z}$ is not available, but we 
do know that the background value of $A_{z}$ is roughly $5.7 \times 10^{-4} \mathrm{~m}^{2} / \mathrm{s}$ and $A_{z}$ is around $(5 \sim 100) \times 10^{-3} \mathrm{~m}^{2} / \mathrm{s}$ in the KPP mixing layer (Chapter 2). $\epsilon$ due to the background value of $A_{z}$ is $0.2 \mathrm{TW}$ in the $\mathrm{ECCO} 2$ state estimate. Assuming that the KPP mixing layer is $100 \mathrm{~m}$ at all the time steps and $A_{z}$ in the mixing layer is always $5 \times 10^{-3} \mathrm{~m}^{2} / \mathrm{s}$, the dissipation in the KPP mixing layer is roughly $1 \mathrm{TW}$ in the ECCO2 state estimate. The contribution of biharmonic viscosity to the kinetic energy dissipation is even larger than the contribution of vertical viscosity (D. Menemenlis 2012, personal communication).

\subsection{Results}

\subsubsection{Global pattern of eddy-mean flow interaction}

Figure 3-4 shows the spatial pattern of eddy-mean flow interaction due to eddy density fluxes (the $B$ terms). The patterns of the $B$ terms are dominated by large magnitude in the Southern Ocean, north of $40^{\circ} N$ in the Atlantic Basin, in the western boundary current regions and in the subtropical gyre. In most of these areas, eddies grow through the interaction with the mean flow $\left(B_{E A P E}>0\right)$ and the mean flow releases available potential energy by interacting with eddies $\left(B_{M A P E}<0\right)$. However, in the eastern part of the Kuroshio and Gulf Stream extension regions, eddies lose energy and the mean flow gains it.

To determine the realism of these patterns, we compare our results with previous modeling studies. The overall pattern of $B_{E K E}$ in the North Atlantic is consistent with that in Zhai and Marshall (2012). von Storch et al. (2012) presented the spatial pattern of $B_{E K E}$ and $B_{E A P E}$ from a $1 / 10^{\circ}$ global simulation. Though their timevarying flow includes variability with periods from 10 minutes to 10 years and our 

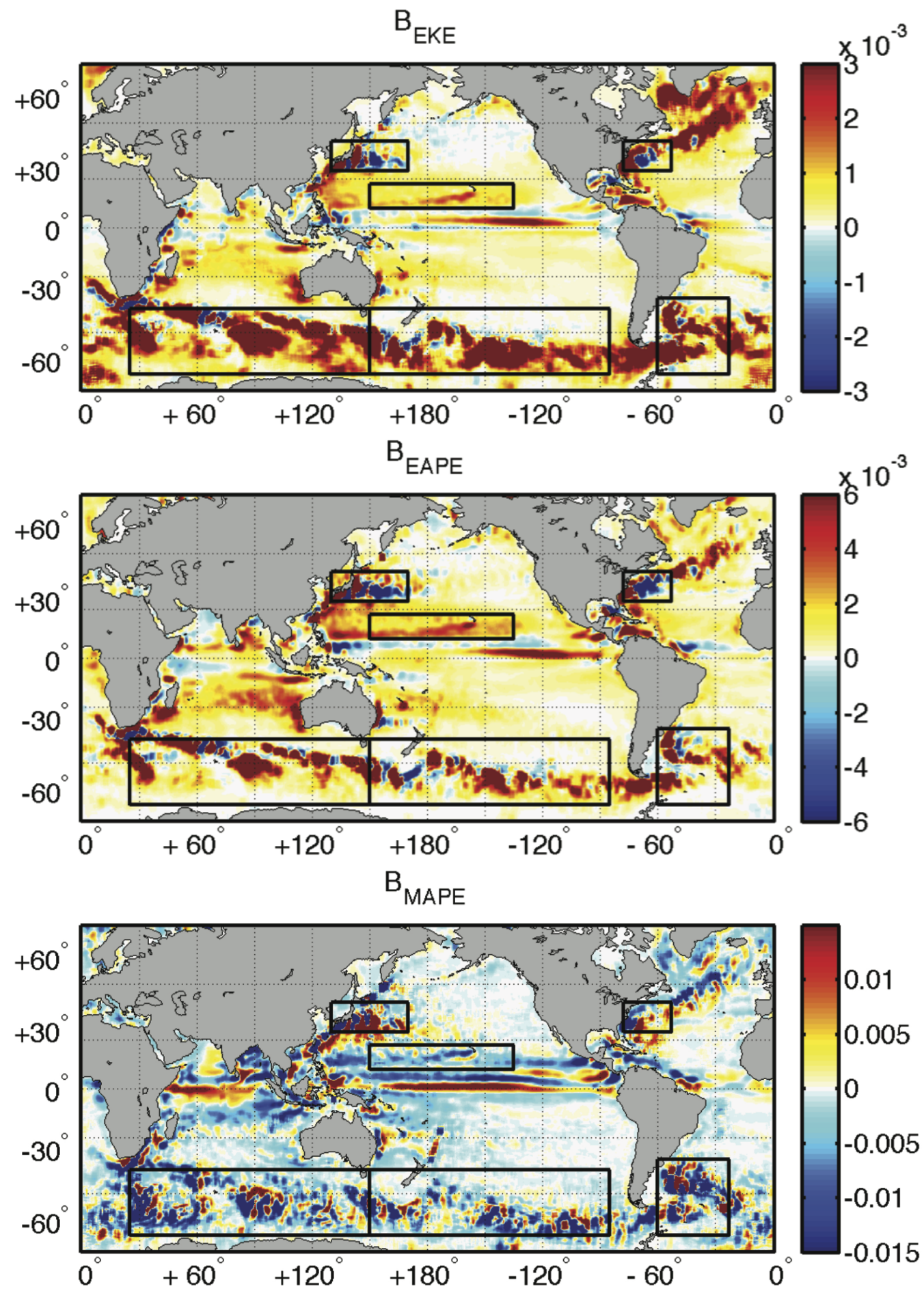

Figure 3-4: The 3-degree running averaged $B_{E K E}, B_{E A P E}$ and $B_{M A P E}$ integrated over the whole water column $\left(W / m^{2}\right)$. These terms describe energy change rates due to eddy-mean flow interaction through eddy density fluxes (Table 3.1). Positive (negative) $B_{E A P E}\left(B_{M A P E}\right)$ means eddies (mean flow) gain (releases) potential energy through this process. Positive $B_{E K E}$ means EAPE is converted to EKE. Magnitudes in the six black boxes are large. 
time-varying flow includes variability with periods from 3 days to 16 years, the spatial patterns of the vertically integrated $B_{E K E}$ and $B_{E A P E}$ in our study is similar to theirs: values are large in the Southern Ocean and western boundary currents and are small in the subpolar gyres; negative spots occur in the western bounder extension regions.

The similarity between the $B_{E K E}$ and $B_{E A P E}$ patterns in Figure 3-4 suggests that part of $B_{E A P E}$ transfers to EKE through the term $B_{E K E}$, which is consistent with baroclinic instability theory (Pedlosky, 1987). However, the globally integrated $B_{E A P E}(0.5 T W)$ is larger than the globally integrated $B_{E K E}(0.3 T W)$; thus only part of the energy extracted by EAPE from MAPE is used to support the EKE growth and the remaining part is used to balance other terms in the EAPE budget. However, the detailed pathway of the energy transferred to EAPE from MAPE $\left(B_{E A P E}\right)$ in the realistic eddy-permitting models is still largely unknown.

Figure 3-5 shows the spatial pattern of vertically integrated eddy-mean flow energy exchanges due to eddy momentum fluxes (the $C$ terms). The patterns are dominated by large magnitude in the western boundary currents and the Southern Ocean and by small magnitude elsewhere. Eddies gain kinetic energy in most areas of the western boundary currents and many spots in the Southern Ocean $\left(C_{E K E}>0\right)$, but they lose kinetic energy in many places in the Southern Ocean $\left(C_{E K E}<0\right) . C_{M K E}$ also has a sequence of positive and negative values in the Southern Ocean. This phenomenon has been identified in previous observation and modeling studies (e.g. Johnson et al., 1992; Morrow et al., 1992; Wilkin and Morrow, 1994).

We find that, from a global integral perspective, eddies gain kinetic energy through $C_{E K E}$ at $0.1 \mathrm{TW}$ and the mean flow releases kinetic energy through $C_{M K E}$ at the same rate. To put this number into context, it is roughly $12 \%$ of the wind power input into the time-mean surface geostrophic flow and it is one third of the globally integrated $B_{E K E}$. In a local region, part of the wind power input into geostrophic flow 


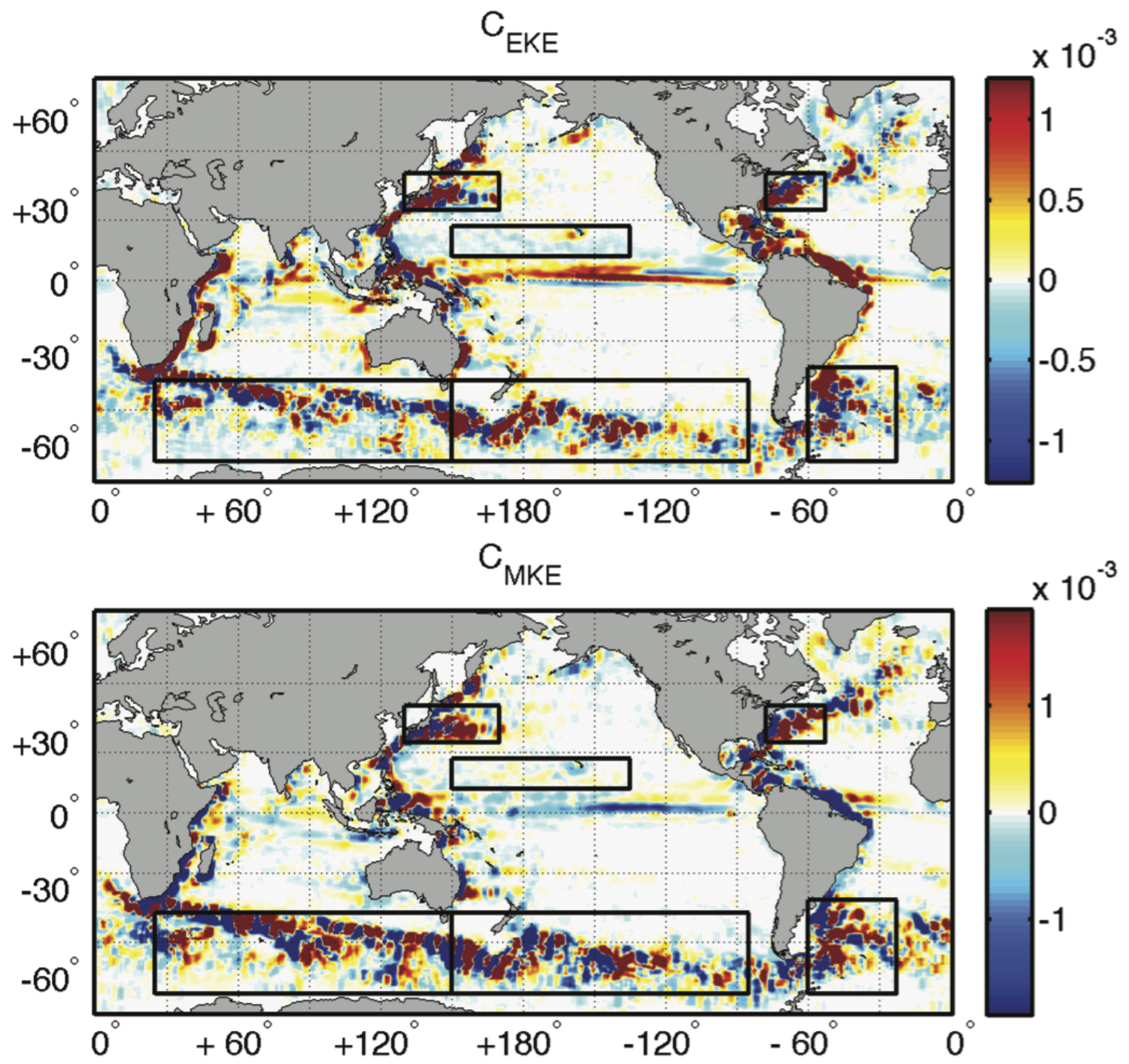

Figure 3-5: The 3-degree running averaged $C_{E K E}$ (upper) and $C_{M K E}$ (lower) integrated over the whole water column $\left(W / m^{2}\right)$. These two terms are about the energy change rates due to eddy-mean flow interaction through eddy momentum fluxes (Table 3.1). Positive (negative) $C_{E K E}\left(C_{M K E}\right)$ means eddies (mean flow) gain (releases) kinetic energy through this process. Their magnitudes are large in the western boundary currents and the Southern Ocean.

is converted to potential energy (Roquet et al., 2011) and then can be released from MAPE through $B_{M A P E}$ and sustain the eddy growth. The other part of the wind power input is transformed to pressure work (Roquet et al., 2011), which can change the local MKE budget and influence the energy released from MKE through $C_{M K E}$. 
Our calculation suggests that a major portion of the wind power input is used to sustain the $B_{M A P E}$ and $B_{E A P E}$ terms, but the contribution of the wind power input to the $C$ terms is also not negligible.

\section{Lorenz energy cycle comparison}

Now consider the energy pathway due to eddy-mean flow interaction integrated over the entire global ocean. The MAPE, EAPE, MKE and EKE reservoirs in a three-dimensional volume are defined as

$$
\begin{aligned}
M A P E & =\iiint_{V} \chi_{\text {mean }}(x, y, z) d \nu \\
E A P E & =\iiint_{V} \chi_{e d d y}(x, y, z) d \nu \\
M K E & =\iiint_{V} e_{\text {mean }}^{k}(x, y, z) d \nu \\
E K E & =\iiint_{V} e_{\text {eddy }}^{k}(x, y, z) d \nu
\end{aligned}
$$

where $V$ is a three-dimensional volume in the ocean, such as a region or the entire global ocean.

Figure 3-6 shows a subset of the traditional Lorenz energy cycle diagram (Lorenz, 1955). von Storch et al. (2012) compared the Lorenz energy cycle in the global ocean, estimated from an eddying model, with the atmospheric one. Our estimate is similar to theirs in several aspects. First, the arrow direction in these two estimates are the same. Both estimates show that the arrow direction is not entirely the same as that in the atmosphere, in which eddy kinetic energy is converted to mean kinetic energy. One caveat here is that eddies in the atmosphere are defined as deviations from the zonal mean, whereas oceanic eddies in von Storch et al. (2012) and this chapter are time-varying flows. Second, the numbers in these two estimates are on the same 
(a) $\mathrm{ECCO} 2$

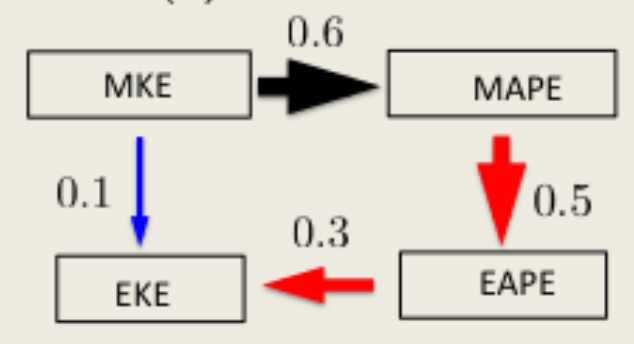

(b) STORM/NCEP

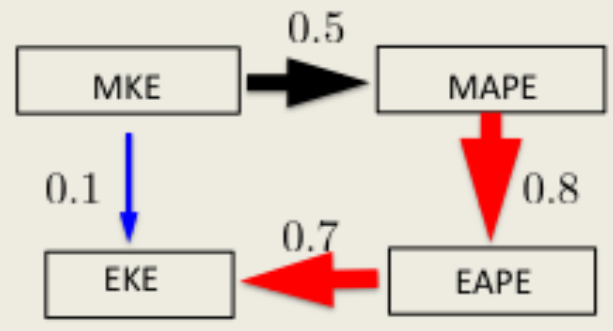

Figure 3-6: The global energy diagram about eddy-mean flow interaction in $T W$ based on the ECCO2 state estimate (a) and the estimate from von Storch et al. (2012) using the STORM/NCEP simulation (b). MAPE, EAPE, MKE and EKE in the boxes are defined in Eqs. 3.26-3.29. The number between MAPE and EAPE in both $(a)$ and $(b)$ is the global integral of $B_{E A P E}$, not $B_{M A P E}$. As mentioned previously in the text, the globally integrated $B_{E A P E}+B_{M A P E}$ is only approximately zero (Section 3.6.2).

order of magnitude. However, our estimate has smaller values for the baroclinic energy pathway $M A P E \rightarrow E A P E \rightarrow E K E$. This may be due in part to differences in model configuration and definitions of eddies. The ECCO2 state estimate is data constrained (Chapter 2), whereas the STORM/NCEP simulation is not. Their timevarying flows include motions with period of 10 minutes $\sim 10$ years, whereas ours include motions with period of 3 days $\sim 16$ years.

\subsubsection{Regional energy routes of eddy-mean flow interaction}

The energy route through eddy-mean flow interaction in specific regions is generally different from the global diagram in Figure 3-6. Next we describe the energy routes through eddy-mean flow interaction in some energetic regions. These regional energy routes (Figures 3-7, 3-8 and 3-9) are the integral of the energy diagram shown in Figure 3-1 over selected regions; however, the energy exchange rates between MKE an MAPE $\left(B_{M K E}\right)$ are not included in these regional energy routes, as we focus on eddy-mean flow interaction. 


\section{Southern Ocean}

The Southern Ocean plays a key role in the global energy budget. The Southern Ocean receives more than $75 \%$ of the total global wind power input (Roquet et al., 2011). The surface westerly wind stress in the Southern Ocean drives surface water northward and thus the water below the surface is brought upward to conserve mass. Isopycnals are thus tilted upward towards the pole and the Deacon cell meridional overturning circulation is formed and further maintained by the surface buoyancy forcing (e.g. Doos and Webb, 1994; Marshall and Radko, 2003; Thompson, 2008). These previous studies agree that available potential energy stored in these tilted isopycnals can be released and used to generate eddies through baroclinic instability. On the other hand, previous observation and modeling work suggests that eddies generated through baroclinic instability in the Southern Ocean can intensify the mean flow through the convergence of eddy momentum fluxes $\left(C_{M K E}>0\right)$ in some regions and decelerate the mean flow through the opposite process $\left(C_{M K E}<0\right)$ in some other regions (e.g. Johnson et al., 1992; Morrow et al., 1992; McWilliams et al., 1978; Wilkin and Morrow, 1994; Lenn et al., 2011).

The eddy-mean flow interaction in the Southern Ocean in the ECCO2 state estimate is consistent with studies summarized above in three aspects. First, in the ECCO2 state estimate, energy is released from the mean available potential energy stored in the tilted isopycnals and eddies are generated $\left(B_{E K E}>0\right.$ and $\left.B_{M A P E}<0\right)$. Second, the gain rate of EKE from EAPE $\left(B_{E K E}\right)$ in the Southern Ocean is roughly half of its globally integrated value, suggesting the key role of the Southern Ocean in the global energy cycle. Third, eddies drive the mean flow through eddy momentum fluxes in some patches $\left(C_{M K E}>0\right)$ and decelerate the mean flow in some other patches $\left(C_{M K E}>0\right)$.

We also identify several new aspects about the eddy-mean flow interaction in the 
Southern Ocean, summarized in Figure 3-7. First, the negative and positive patches of $C_{M K E}$ integrated over the three Southern Ocean boxes shown in Figure 3-5 mostly cancel out. The contribution of $C_{E K E}$ to the eddy growth in the Southern Ocean is an order of magnitude smaller than the contribution of $B_{E A P E}$. Second, energy released from the mean flow through $B_{M A P E}$ is $214 \mathrm{GW}$, but only $139 \mathrm{GW}$ transfers to the EAPE reservoir through the term $B_{E A P E}$. Thus, two thirds of energy released from the available potential energy stored in the tilted isopycnals is used to support the eddy growth in the Southern Ocean and the rest of the energy released from the mean flow is transported out of the domain through the divergence term $B_{M A P E}+B_{E A P E}$. This indicates that eddy-mean flow interaction in the Southern Ocean is non-local to some extent. The nonlocal nature arises from the spatial inhomogeneity of the eddy density fluxes and the mean flow, as it is

$$
B_{M A P E}+B_{E A P E}=\frac{g}{n_{0}} \nabla_{H} \cdot\left(\overline{\mathbf{u}_{\mathbf{H}}^{\prime} \rho^{\prime}} \overline{\rho^{*}}\right) \neq 0 .
$$

Both the mean flow, dominated by fronts and jet features, and the time-mean observed eddy heat fluxes in the Southern Ocean have rich small-scale variations (e.g. Lenn et al., 2011).

The energy routes in the Indian Sector, the Pacific Sector and the Atlantic Sector of the Southern Ocean are not entirely the same (Figure 3-8). In all the three sectors, the contribution of the $C$ terms to the eddy growth is negligible compared to the contribution of the $B$ terms. In the Indian Sector (Atlantic Sector), roughly 70\% (40\%) of the energy released from the MAPE reservoir is used to sustain the eddy growth in the same region; in the Pacific Sector, however, roughly $90 \%$ of the energy released from the MAPE reservoir is used to sustain the eddy growth in the same sector. The mechanism for the differences between these sectors is still to be determined. 
(a) Southern Ocean

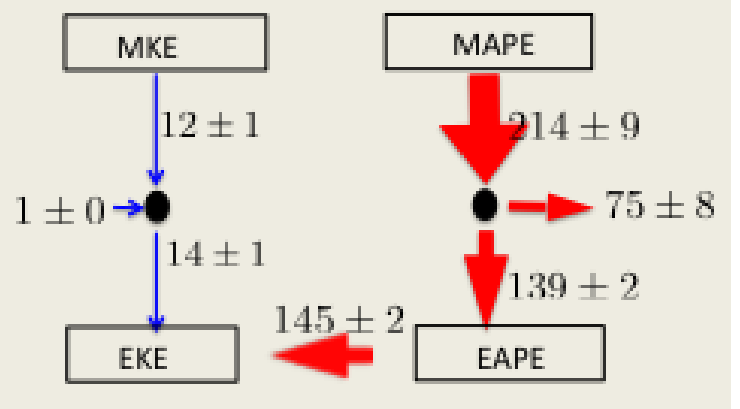

(c) Kuroshio Extension

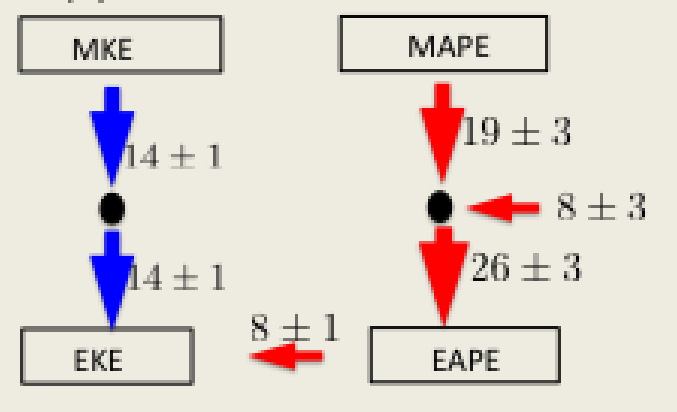

(b) Subtropical gyre

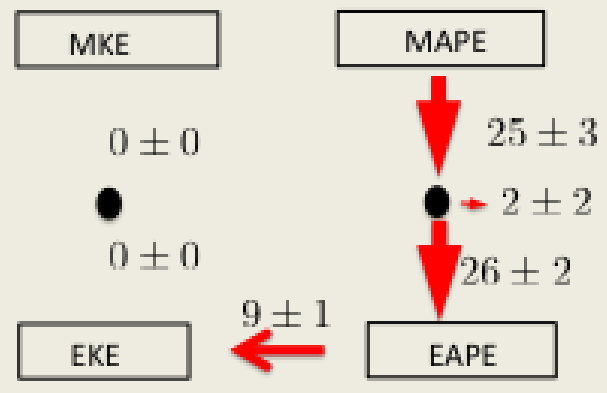

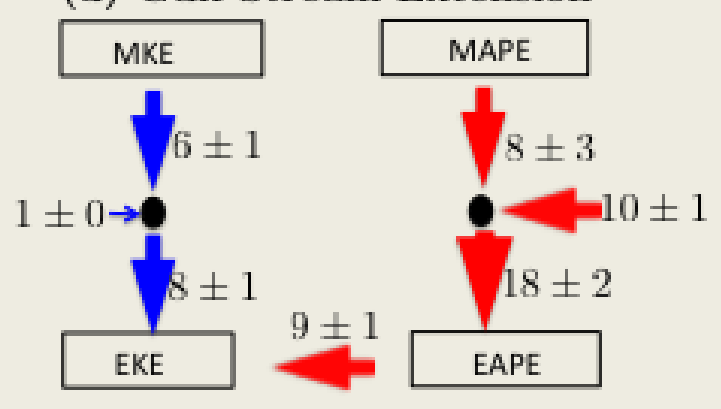

Figure 3-7: The energy diagram in $10^{9} W(G W)$ (Figure 3-1) in the three boxes in the Southern Ocean (a) and in the three boxes in the northern hemisphere (b, c, d) shown in Figure 3-4. In diagram a, only part of the energy released from MAPE through eddy-mean flow interaction supports local eddy energy growth. In diagram $\mathrm{b}$, eddy-mean flow interaction is local. In diagrams $\mathrm{c}$ and $\mathrm{d}$, the contribution of $B$ and $C$ terms to the eddy growth is on the same order of magnitude. Errors shown here are one standard error, as that in Table 3.2. Two standard errors correspond to $95 \%$ confidence level. MAPE, EAPE, MKE and EKE in the boxes are defined in Eqs. 3.26-3.29.

We also find that, compared to eddy-mean flow interaction through density fluxes, horizontal advection contributes much less to the eddy energy change. First, the EKE change rate due to horizontal advection in each sector is less than $4 G W$ and the EKE change rate due to horizontal advection integrated over these three sectors is $0 G W$. Second, the EAPE change rate due to horizontal advection in each sector is less than $2 G W$. The divergent eddy heat fluxes are strong in the Southern Ocean (Jayne and Marotzke, 2002) and thus the divergent eddy density fluxes are also strong here, 
(a) Indian Sector

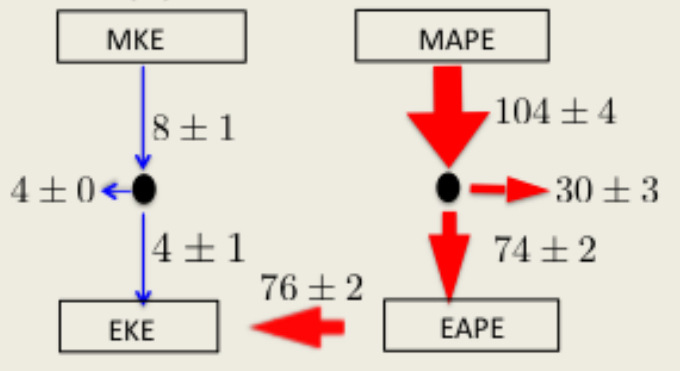

(b) Pacific Sector

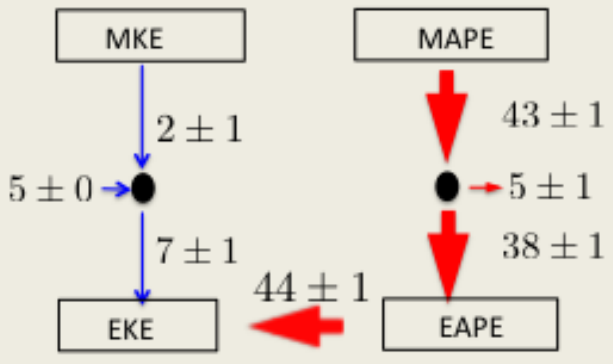

(c) Atlantic Sector

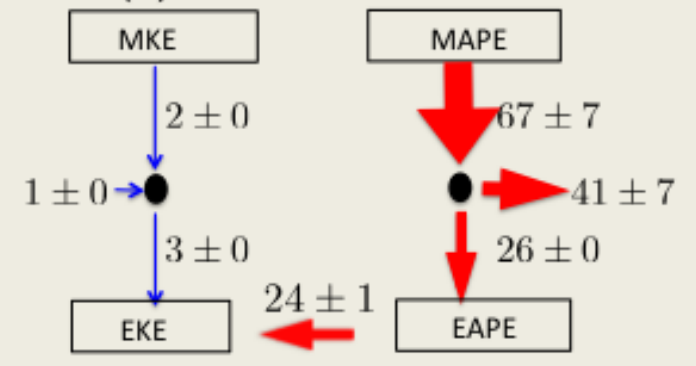

Figure 3-8: The energy diagram in $10^{9} W(G W)$ (Figure 3-1) in the Indian Sector (diagram a), the Pacific Sector (diagram b) and the Atlantic Sector (diagram c) of the Southern Ocean, denoted by the boxes in Figure 3-4. The contribution of the $C$ terms to eddy growth is negligible. In the Indian and Atlantic Sectors, only part of the energy released from MAPE through eddy-mean flow interaction supports the eddy energy growth in the same domain. In the Pacific Sector, roughly all the MAPE released supports eddy energy growth in the same domain. Errors shown here are one standard error, as that in Table 3.2. Two standard errors correspond to $95 \%$ confidence level. MAPE, EAPE, MKE and EKE in the boxes are defined in Eqs. 3.26-3.29.

assuming that the effect of salinity on the density fluxes is small. Thus, $B_{E A P E, R}$ is probably much smaller than $B_{E A P E, D}$. Consistently, $B_{E A P E}$ is dominated by $B_{E A P E, D}$ and $B_{E A P E}$ roughly balances $B_{E K E}$ in all the three sectors.

\section{Subtropical gyres}

Figure 3-7 shows the energy route through eddy-mean flow interaction in a midocean patch in the subtropical gyre, indicated by the black box in the mid-ocean of the North Pacific in Figure 3-4. In this region, eddy-mean flow interaction due to eddy 
momentum fluxes is negligible (the $C$ terms are zero). Approximately all the energy released from MAPE is used to sustain the local EAPE growth and little energy is exported elsewhere through $B_{M A P E}+B_{E A P E}$. Thus, eddy-mean flow interaction in this patch is local and the local assumption used in previous studies (e.g. Gill et al., 1974; Tulloch et al., 2011; Arbic and Flierl, 2004) is accurate in this region. We also find that the EKE change rate due to horizontal advection in this region is 0 $G W$. Also $35 \%$ of $B_{E A P E}$ balances $B_{E K E}$ and $9 \%$ of $B_{E A P E}$ balances the horizontal advection of EAPE; thus more than half of $B_{E A P E}$ balances other terms in the EAPE budget. Whether the results in this patch are representative of the subtropical gyres in other ocean basins is to be determined.

\section{Western boundary extensions}

The energy routes in the Kuroshio and Gulf Stream Extension regions, denoted by the two black boxes around $35^{\circ} \mathrm{N}$ in Figure 3-4, are shown in Figure 3-7. First, $B_{E K E}$ is positive in the western part of the extension and negative in the eastern part, whereas $C_{M K E}$ is positive in the eastern part of the extension and negative in the western part (Figures 3-4 and 3-5). These features are consistent with previous observation, modeling and theoretical studies (e.g. Waterman and Jayne, 2011; Nishida and White, 1982; Eden et al., 2007; Zhai and Marshall, 2012; Hall, 1991). The consistency indicates that the energetics of the ECCO2 simulation are reasonable in these areas. Second, in the ECCO2 state estimate, the EAPE loss rate through horizontal advection is $6 G W(5 G W)$ in the Kuroshio (Gulf Stream) Extension region, which is non-negligible compared to $B_{E A P E}$. Previous modeling and mooring analysis shows that the rotational eddy heat fluxes in the Gulf Stream Extension region are large (Cronin and Watts, 1996; Jayne and Marotzke, 2002). This suggests that a non-negligible part of $B_{E A P E}$ is $B_{E A P E, R}$, which may balance the EKE loss through horizontal advection in this region. 
The energy route in these extension regions is different from those in the Southern Ocean and the subtropical gyre in the following aspect (Figure 3-7). Eddies integrated over the whole extension regions gain energy from the mean flow through both the $B$ and $C$ terms, whose contribution to the EKE growth is on the same order of magnitude. The energy input through the boundaries of this region $\left(B_{M A P E}+B_{E A P E}\right)$ is also not negligible.

Here we discuss the energy route in the Kuroshio Extension region in more detail by dividing it into two subregions (Figure 3-9). Figure 3-7 (c) shows that, in the Kuroshio Extension region, energy is transferred from MAPE to EAPE with some energy input from the boundaries of the region and a small portion being converted to EKE. The EKE loss rate through horizontal advection in this region is negligible (1 $G W$ ) compared to the gain rate of EKE from EAPE and MKE. More detailed examination shows that the energy pathway in Figure 3-7 (c) is essentially the average over two different dynamical regimes (Figure 3-9). In the western half, energy is transferred from both the kinetic energy and available potential energy reservoir in the mean flow to the eddy energy reservoir. The energy input from other regions is small and the eddy-mean flow interaction is approximately local. Only $26 \%$ of EAPE extracted from the mean flow is converted to EKE, and the EKE loss rate from horizontal advection is $37 \%$ of the gain rate of EKE from EAPE and MKE. By contrast, energy in the eastern half is converted from EAPE to MAPE; however, this is not the local baroclinic instability mechanism operating in reverse, as a large portion of the energy fed into MAPE is supplied from elsewhere through the divergence term and most of the EAPE loss to MAPE is not supplied by the local EKE reservoir. The gain rate of EKE through horizontal advection in the eastern half is three times larger than the EKE loss rate to EAPE. Note that, compared to the energy route in the eastern half, the energy route in the western half resembles more the energy route 
for the whole Kuroshio Extension region, shown in Figure 3-7 (c).

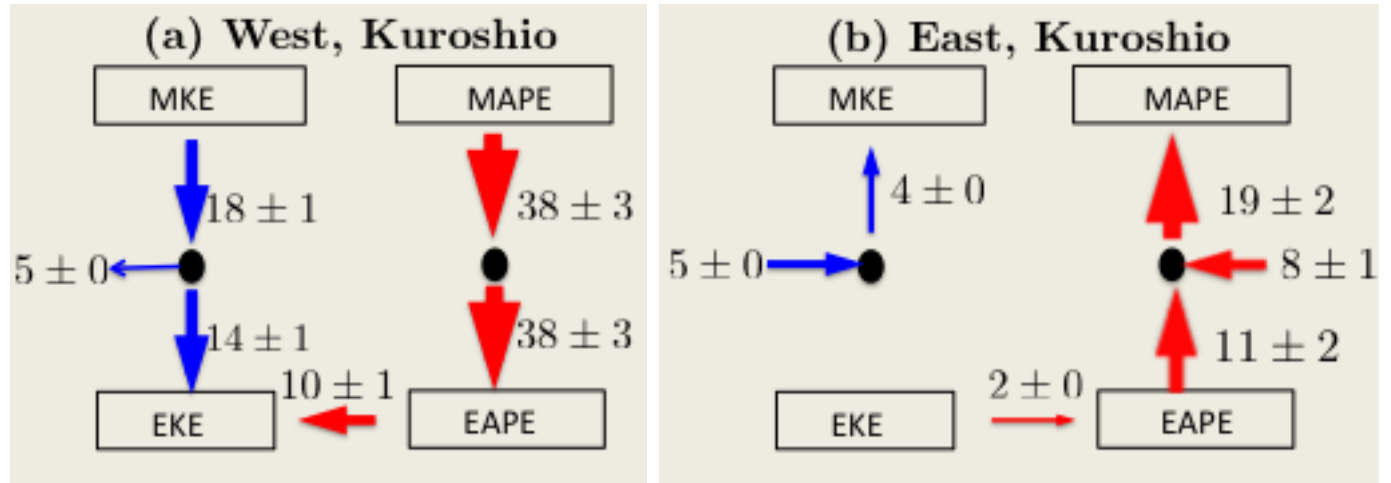

Figure 3-9: The energy diagram in $10^{9} W(G W)$ (Figure 3-1) in the western half (a) and eastern half (b) of the Kuroshio Extension region, denoted by the box in Figure 3-4. In (a), energy is transferred from the mean flow to eddies through both the $B$ and $C$ terms; in (b), energy is transferred from EAPE to MAPE. Errors shown here are one standard error, as that in Table 3.2. Two standard errors correspond to $95 \%$ confidence level. MAPE, EAPE, MKE and EKE in the boxes are defined in Eqs. 3.26-3.29.

To our best knowledge, neither a complete diagnosis of the eddy-mean flow interaction terms in Table 3.1 nor a complete theory exist in previous studies about energetics in the Kuroshio Extension region. However, using two-year mooring data at one site in the Kuroshio Extension region $\left(35^{\circ} N, 152^{\circ} E\right)$, Hall (1991) found that $B_{E K E}<0$ and $B_{E A P E}<0$ at 350 dbar and $C_{E K E}$ is generally negative at this site. Waterman and Jayne (2011) found that, by analyzing the potential vorticity and enstrophy budgets in an idealized two-layer model, eddies can drive the mean flow in the eastern part of the Kuroshio Extension through nonlinear eddy rectification processes due to localized forcing. Different from Waterman and Jayne (2011), we find that both eddies and the energy input through the boundaries contribute to the APE increase in the mean flow in the eastern part of the Kuroshio Extension region (Figure 3-9). A complete theory about the energy pathways in Figure 3-9 does not exist. Whether these energetic features exist in the instability processes due to the 
localized forcing (e.g. pulse instabilities) is still not known (e.g. Farrell, 1982; Helfrich and Pedlosky, 1993, 1995).

\subsection{Conclusions}

This chapter provides a global description about the energetics of eddy-mean flow interaction in the ECCO2 state estimate. From a global integral perspective, the dominant energy route through eddy-mean flow interaction is from mean available potential energy to eddy available potential energy then to eddy kinetic energy; however, the energy transfer from the mean kinetic energy to eddy kinetic energy is also not negligible (Figure 3-6).

The energetics of eddy-mean flow interaction vary geographically. The mean flow releases energy through eddy-mean flow interaction in most regions, but gains energy in some other regions. Interactions due to eddy density fluxes are pronounced in the Southern Ocean, western boundary extension regions and the subtropical gyres while interactions due to eddy momentum fluxes play a large role in the Southern Ocean and western boundary current regions. The interaction is approximately local in the selected subtropical gyre region, but it is nonlocal in the Southern Ocean, where the oceanic circulation is less spatially homogeneous. Energetics in the eastern half and the western half of the Kuroshio Extension region are very different. In the western half, the mean flow is both baroclinically and barotropically unstable and most energy released from the mean flow transfers to eddies; in the eastern half, eddy energy transfers back to the mean flow and eddy-mean flow interaction is nonlocal, as the divergence terms are non-negligible.

Some tasks for the future are (1) to explore the sensitivity of the results to the spatial resolution, record length and the region size by examining regions with dif- 
ferent sizes and carrying out longer and higher resolution numerical simulations, (2) to develop a diagnostic framework based on a more accurate definition of available potential energy, (3) to diagnose other terms in the energy budgets and evaluate the vorticity, enstrophy and momentum budgets in order to have a more complete description of eddy-mean flow interaction in the global ocean, and (4) to partition the contribution of oceanic variability at different spatial and time scales to the eddymean flow interaction process. Besides these future tasks, the results in this chapter also raise more puzzles:

- Small-scale features are prevalent in the temporally averaged sea surface height field over four years (Wunsch, 2010). Here we find that small-scale features are also prevalent in the time-mean eddy-mean flow interaction patterns. Do these features become less pronounced if we use a longer record?

- In our current estimate, one third of the energy released from the APE in the mean flow of the three boxes in the Southern Ocean moves to other regions through the divergence term. Assuming this result is not sensitive to the model resolution, record length and diagnostic framework, it is important to study (1) what causes the non-local nature of eddy-mean flow interaction in the Southern Ocean? (2) What are the consequences of this non-local nature on jet behaviors, eddy characteristics, and their contributions to oceanic circulation, mixing and transport in the Southern Ocean? (3) What is the impact of the non-local nature on the time-mean circulation, eddy field and energetics in other regions? (4) Is it important to develop parameterization schemes not using the local assumption? 


\subsection{Appendix}

\subsubsection{Governing equations for kinetic energy}

As discussed in Chapter 2, the momentum equations in the $\mathrm{x}$ and $\mathrm{y}$ directions in the ECCO2 state estimate are

$$
\begin{aligned}
& \frac{\partial u}{\partial t}+\nabla \cdot(u \mathbf{u})-f v=-\frac{1}{\rho_{0}} \frac{\partial}{\partial x} p+D_{u} \\
& \frac{\partial v}{\partial t}+\nabla \cdot(v \mathbf{u})+f u=-\frac{1}{\rho_{0}} \frac{\partial}{\partial y} p+D_{v},
\end{aligned}
$$

where

$$
D_{u}=\frac{\partial}{\partial z} A_{z} \frac{\partial u}{\partial z}+A_{4} \nabla_{h}^{4} u \text { and } D_{v}=\frac{\partial}{\partial z} A_{z} \frac{\partial v}{\partial z}+A_{4} \nabla_{h}^{4} v
$$

respectively representing the change rates of momentum in the $\mathrm{x}$ and $\mathrm{y}$ direction due to vertical and horizontal friction. $\rho_{0}$ is constant reference density, $p$ is the hydrostatic

pressure, $A_{z}$ is the vertical viscosity and $A_{4}$ is the horizontal biharmonic viscosity. $u$ is the zonal velocity and $v$ is the meridional velocity. $\mathbf{u}$ denotes the velocity vector and $\nabla \cdot$ is the divergence operator:

$$
\mathbf{u}=u \widehat{i}+v \widehat{j}+w \widehat{k}, \nabla \cdot=\frac{\partial}{\partial x} \widehat{i}+\frac{\partial}{\partial y} \widehat{j}+\frac{\partial}{\partial z} \widehat{k}
$$

Multiply Eqs. 3.30 and 3.31 by $\bar{u}$ and $\bar{v}$ respectively, sum them together and perform a temporal average to obtain the mean kinetic energy equation:

$\frac{\partial}{\partial t} e_{\text {mean }}^{k}+\nabla \cdot\left(\overline{\mathbf{u}} e_{\text {mean }}^{k}\right)+\nabla \cdot(\overline{\mathbf{u}} \bar{p})=-g \bar{\rho} \bar{w}-\rho_{0}\left[\bar{u} \nabla \overline{\cdot\left(\mathbf{u}^{\prime} u^{\prime}\right)}+\bar{v} \nabla \cdot \overline{\left(\mathbf{u}^{\prime} v^{\prime}\right)}\right]+\rho_{0}\left(\bar{u} \overline{D_{u}}+\bar{v} \overline{D_{v}}\right)$ 
where

$$
e_{\text {mean }}^{k}(x, y, z)=\frac{1}{2} \rho_{0}\left(\bar{u}^{2}+\bar{v}^{2}\right)
$$

and $\rho$ is the in-situ density.

Multiply Eqs. 3.30 and 3.31 by $u^{\prime}$ and $v^{\prime}$ respectively, sum them together and perform a temporal average to obtain the eddy kinetic energy equation:

$$
\begin{aligned}
\left.\frac{\partial}{\partial t} e_{e d d y}^{k}+\nabla \cdot \overline{\left[\mathbf{u} \frac{1}{2} \rho_{0}\left(u^{\prime 2}+v^{\prime 2}\right)\right]}+\nabla \cdot \overline{\left(\mathbf{u}^{\prime} p^{\prime}\right.}\right)= & -g \overline{\rho^{\prime} w^{\prime}}-\overline{\rho_{0}\left(u^{\prime} \mathbf{u}^{\prime} \cdot \nabla \bar{u}+v^{\prime} \mathbf{u}^{\prime} \cdot \nabla \bar{v}\right)} \\
& +\rho_{0}\left(\overline{u^{\prime} D_{u}^{\prime}}+\overline{v^{\prime} D_{v}^{\prime}}\right)
\end{aligned}
$$

where

$$
e_{e d d y}^{k}(x, y, z)=\frac{1}{2} \rho_{0} \overline{\left(u^{\prime 2}+v^{\prime 2}\right)}
$$

According to Eqs. 3.4 and 3.5, we can decompose the pressure and in-situ density as follows:

$$
p(x, y, z, t)=p^{*}(x, y, z, t)+\tilde{p}(z), \rho(x, y, z, t)=\rho^{*}(x, y, z, t)+\tilde{\rho}(z)
$$

also noting that the hydrostatic approximation is employed in the ECCO2 state estimate, we obtain

$$
\nabla \cdot(\overline{\mathbf{u}} \bar{p})+g \bar{\rho} \bar{w}=\nabla \cdot\left(\overline{\mathbf{u}} \overline{p^{*}}\right)+g \overline{\rho^{*}} \bar{w} .
$$

Thus, we can write the equations for kinetic energy (Eqs. 3.33 and 3.35) into the following form:

$$
\frac{\partial}{\partial t} e_{\text {mean }}^{k}+\nabla \cdot\left(\overline{\mathbf{u}} e_{\text {mean }}^{k}\right)+\nabla \cdot\left(\overline{\mathbf{u}} \overline{p^{*}}\right)=-\underbrace{g \overline{\rho^{*}} \bar{w}}_{B_{M K E}} \underbrace{-\rho_{0}\left[\bar{u} \nabla \overline{\cdot\left(u^{\prime} \mathbf{u}^{\prime}\right)}+\bar{v} \nabla \overline{\cdot\left(v^{\prime} \mathbf{u}^{\prime}\right)}\right]}_{C_{M K E}}+\underbrace{\rho_{0}\left(\bar{u} \overline{D_{u}}+\bar{v} \overline{D_{v}}\right)}_{D_{M K E}},
$$


$\left.\left.\frac{\partial}{\partial t} e_{e d d y}^{k}+\nabla \cdot \overline{\left[\mathbf{u} \frac{1}{2} \rho_{0}\left(u^{\prime 2}+v^{\prime 2}\right)\right.}\right]+\nabla \cdot \overline{\left(\mathbf{u}^{\prime} p^{\prime}\right.}\right)=\underbrace{-g \overline{\rho^{\prime} w^{\prime}}}_{B_{E K E}} \underbrace{-\rho_{0}\left(\overline{u^{\prime} \mathbf{u}^{\prime}} \cdot \nabla \bar{u}+\overline{v^{\prime} \mathbf{u}^{\prime}} \cdot \nabla \bar{v}\right)}_{C_{E K E}}+D_{E K E}$,

where

$$
D_{E K E}=\rho_{0}\left(\overline{u^{\prime} D_{u}^{\prime}}+\overline{v^{\prime} D_{v}^{\prime}}\right)
$$

Note that the global integral of $C_{M K E}+C_{E K E}$ is zero, as it has the divergence form:

$$
\iiint_{V}\left(C_{E K E}+C_{M K E}\right) d \nu=-\rho_{0} \iiint_{V}\left(\left[\nabla \cdot \overline{\left(\bar{u} u^{\prime} \mathbf{u}^{\prime}\right)}+\nabla \cdot \overline{\left(\bar{v} v^{\prime} \mathbf{u}^{\prime}\right)}\right]\right) d \nu=0 .
$$

\subsubsection{Governing equations for available potential energy}

A prerequisite to deriving the MAPE and EAPE equations consistent with the ECCO2 state estimate is to obtain the in-situ density equation consistent with the ECCO2

state estimate. The potential temperature $(\theta)$ and salinity $(S)$ equations in the ECCO2 state estimate are

$$
\begin{gathered}
\frac{d \theta}{d t}=\frac{\partial \theta}{\partial t}+\left(u \frac{\partial}{\partial x}+v \frac{\partial}{\partial y}+w \frac{\partial}{\partial z}\right) \theta=H_{\theta} \\
\frac{d S}{d t}=\frac{\partial S}{\partial t}+\left(u \frac{\partial}{\partial x}+v \frac{\partial}{\partial y}+w \frac{\partial}{\partial z}\right) S=H_{S}
\end{gathered}
$$

where

$$
\begin{gathered}
H_{\theta}=\frac{\partial}{\partial z}\left(K_{z} \frac{\partial \theta}{\partial z}\right)+\left\{\begin{array}{l}
F_{\theta}(\text { surface level }) \\
\text { penetrating short wave radiation (interior })
\end{array}\right. \\
H_{S}=\frac{\partial}{\partial z}\left(K_{z} \frac{\partial S}{\partial z}\right)+\left\{\begin{array}{l}
F_{S}(\text { surface level }) \\
0 \text { (interior })
\end{array}\right.
\end{gathered}
$$

$F_{\theta}$ and $F_{S}$ represent surface forcing and they are defined in Chapter 2. $K_{z}$ is vertical diffusivity used to parameterize the mixing process in the model and it is spatially and 
temporally varying. As discussed in Chapter 2, the equation of state in the ECCO2 state estimate is

$$
\rho(x, y, z, t)=\rho\left(\theta, S, \rho_{0} g z\right),
$$

where $\rho$ is the in-situ density; thus

$$
\begin{aligned}
\frac{d \rho}{d t}= & \left(\frac{\partial}{\partial t}+u \frac{\partial}{\partial x}+v \frac{\partial}{\partial y}+w \frac{\partial}{\partial z}\right) \rho \\
= & \left(\frac{\partial \rho}{\partial S}\right)_{\theta, z}\left(\frac{\partial}{\partial t}+u \frac{\partial}{\partial x}+v \frac{\partial}{\partial y}+w \frac{\partial}{\partial z}\right) S+\left(\frac{\partial \rho}{\partial \theta}\right)_{S, z}\left(\frac{\partial}{\partial t}+u \frac{\partial}{\partial x}+v \frac{\partial}{\partial y}+w \frac{\partial}{\partial z}\right) \theta \\
& +w\left(\frac{\partial \rho}{\partial z}\right)_{S, \theta} .
\end{aligned}
$$

Using Eqs. 3.40 and 3.41, we obtain

$$
\frac{\partial \rho}{\partial t}+u \frac{\partial \rho}{\partial x}+v \frac{\partial \rho}{\partial y}+w \frac{\partial \rho}{\partial z}=H_{\rho}+w \widehat{\rho_{z}},
$$

where

$$
\widehat{\rho}_{z}=\left(\frac{\partial \rho}{\partial z}\right)_{S, \theta} .
$$

and

$$
H_{\rho}=\left(\frac{\partial \rho}{\partial \theta}\right)_{S, z} H_{\theta}+\left(\frac{\partial \rho}{\partial S}\right)_{\theta, z} H_{S} .
$$

As discussed in von Storch et al. (2012), the vertical gradient of local potential density is due to the change of salinity and potential temperature with depth at the same location:

$$
\frac{\partial \rho}{\partial z}-\widehat{\rho}_{z}=\left(\frac{\partial \rho}{\partial S}\right)_{\theta, z} \frac{\partial S}{\partial z}+\left(\frac{\partial \rho}{\partial \theta}\right)_{S, z} \frac{\partial \theta}{\partial z}
$$

and thus the time-mean and global mean of the local potential density vertical gra- 
dient is

$$
n_{0}=<\overline{\frac{\partial \rho}{\partial z}-\widehat{\rho_{z}}}>
$$

As defined in the main text (Eqs. 3.4-3.6),

$$
\rho(x, y, z, t)=\tilde{\rho}(z)+\overline{\rho^{*}}(x, y, z)+\rho^{\prime}(x, y, z, t) .
$$

Similarly, we can also decompose $\widehat{\rho}_{z}$ into three parts:

$$
\widehat{\rho_{z}}(x, y, z, t)=<\overline{\widehat{\rho}_{z}}>(z)+{\widehat{\rho_{z}}}^{*}(x, y, z, t)=<\overline{\widehat{\rho}_{z}}>(z)+\overline{{\widehat{\rho_{z}}}^{*}}(x, y, z)+{\widehat{\rho_{z}}}^{\prime}(x, y, z, t)
$$

Substituting Eqs. 3.51 and 3.52 into Eq. 3.46, we obtain the density equation for $\rho^{*}$ :

$$
\frac{\partial}{\partial t} \rho^{*}+\nabla \cdot\left(\mathbf{u} \rho^{*}\right)+w n_{0}=H_{\rho}+w{\widehat{\rho_{z}}}^{*}
$$

Eq. 3.53 can be rewritten as

$$
\frac{\partial}{\partial t}\left(\overline{\rho^{*}}+\rho^{\prime}\right)+\nabla \cdot\left[\mathbf{u}\left(\overline{\rho^{*}}+\rho^{\prime}\right)\right]+w n_{0}=H_{\rho}+w \widehat{\rho}_{z}^{*}
$$

Multiply Eq. 3.54 by $-g \overline{\rho^{*}} / n_{0}$ and then time average, we can get the MAPE equation:

$$
\frac{\partial}{\partial t} \chi_{\text {mean }}+\nabla \cdot\left(\overline{\mathbf{u}} \chi_{\text {mean }}\right)=\underbrace{\overline{\rho^{*}} \nabla \cdot \overline{\left(\mathbf{u}^{\prime} \rho^{\prime} \frac{g}{n_{0}}\right.}}_{B_{M A P E, 0}})+\underbrace{-\frac{g}{n_{0}} \overline{\rho^{*}} \overline{H_{\rho}}}_{B_{M K E} \overline{\rho^{*} \bar{w}}}+R_{D_{M A P E}}
$$

where

$$
\chi_{\text {mean }}(x, y, z)=-\frac{g}{2 n_{0}}\left[\overline{\rho^{*}}(x, y, z)\right]^{2},
$$




$$
R_{x_{\text {mean }, 0}}=-\chi_{\text {mean }} \cdot \frac{\bar{w}}{n_{0}} \frac{\partial n_{0}}{\partial z}-g \overline{\rho *} \overline{w^{\prime} \rho^{\prime}} \frac{\partial}{\partial z}\left(\frac{1}{n_{0}}\right)-\frac{g}{n_{0}} \overline{\rho^{*}} \overline{w \widehat{\rho}_{z}^{*}}
$$

Multiply Eq. 3.54 by $-g \rho^{\prime} / n_{0}$ and then time average, we can get the EAPE equation:

$$
\frac{\partial}{\partial t} \chi_{e d d y}+\nabla \cdot \overline{\left[-\mathbf{u} g \rho^{\prime 2} /\left(2 n_{0}\right)\right]}=\underbrace{\overline{\mathbf{u}^{\prime} \rho^{\prime} \frac{g}{n_{0}}} \cdot \nabla \overline{\rho^{*}}}_{B_{E A P E, 0}}-\underbrace{\left(-g \overline{\rho^{\prime} w^{\prime}}\right)}_{B_{E K E}}-\underbrace{-\frac{g}{n_{0}} \overline{\rho^{\prime} H_{\rho}^{\prime}}}_{D_{E A P E}}+R_{x_{e d d y, 0}},
$$

where

$$
\begin{gathered}
\chi_{e d d y}(x, y, z)=-\frac{g}{2 n_{0}} \overline{\rho^{\prime}(x, y, z, t)^{2}}, \\
R_{x_{e d d y, 0}}=\overline{g \rho^{\prime 2} /\left(2 n_{0}\right) \cdot \frac{w}{n_{0}} \frac{\partial n_{0}}{\partial z}}-\frac{g}{n_{0}} \overline{\rho^{\prime} w \widehat{\rho}_{z}^{*}} .
\end{gathered}
$$

Note that

$$
\iiint_{V}\left(B_{E A P E, 0}+B_{M A P E, 0}\right) d \nu=\iiint_{V}\left(\nabla \cdot \overline{\left(\mathbf{u}^{\prime} \rho^{\prime} \frac{g}{n_{0}}\right.} \overline{\rho^{*}}\right) d \nu=0
$$

where $V$ here refers to global integral. $B_{E A P E, 0}$ and $B_{M A P E, 0}$ can be divided into a horizontal eddy density flux part and a vertical density flux part:

$$
\begin{gathered}
B_{E A P E, 0}=\underbrace{\overline{\mathbf{u}_{\mathbf{H}}^{\prime} \rho^{\prime} \frac{g}{n_{0}}} \cdot \nabla_{H} \overline{\rho^{*}}}_{B_{E A P E}}+\overline{w^{\prime} \rho^{\prime} \frac{g}{n_{0}}} \frac{\partial}{\partial z} \overline{\rho^{*}} \\
B_{M A P E, 0}=\underbrace{\overline{\rho^{*}}}_{B_{M A P E}} \overline{\left.\overline{\nabla_{H} \cdot\left(\overline{\mathbf{u}_{\mathbf{H}}^{\prime} \rho^{\prime} \frac{g}{n_{0}}}\right.}\right)}+\overline{\rho^{*}} \frac{\partial}{\partial z} \overline{\left(w^{\prime} \rho^{\prime} \frac{g}{n_{0}}\right.}),
\end{gathered}
$$

where $\mathbf{u}_{\mathbf{H}}$ is horizontal velocity and

$$
\nabla_{H}=\frac{\partial}{\partial x} \widehat{i}+\frac{\partial}{\partial y} \widehat{j}
$$

This chapter diagnoses $B_{E A P E}$ and $B_{M A P E}$ instead of $B_{E A P E, 0}$ and $B_{M A P E, 0}$, as 
$B_{E A P E}$ and $B_{M A P E}$ are involved in quasi-geostrophic eddy dynamics and can be used to indicate baroclinic instability. For convenience purpose, we write Eqs. 3.55 and 3.57 into the following form:

$$
\begin{gathered}
\frac{\partial}{\partial t} \chi_{\text {mean }}+\nabla \cdot\left(\overline{\mathbf{u}} \chi_{\text {mean }}\right)=\underbrace{\left.\overline{\rho^{*}} \nabla_{H} \cdot \overline{\left(\mathbf{u}_{\mathbf{H}}^{\prime} \rho^{\prime} \frac{g}{n_{0}}\right.}\right)}_{B_{M A P E}}+\underbrace{g \overline{\rho^{*}} \bar{w}}_{B_{M K E}}-\underbrace{\frac{g}{n_{0}} \overline{\rho^{*}} \overline{H_{\rho}}}_{D_{M A P E}}+R_{x_{\text {mean }}}, \\
\frac{\partial}{\partial t} \chi_{e d d y}+\nabla \cdot \overline{\left[-\mathbf{u} g \rho^{\prime 2} /\left(2 n_{0}\right)\right]}=\underbrace{\overline{\mathbf{u}_{\mathbf{H}}^{\prime} \rho^{\prime} \frac{g}{n_{0}}} \cdot \nabla_{H} \overline{\rho^{*}}}_{B_{E A P E}}-\underbrace{\left(-g \overline{\rho^{\prime} w^{\prime}}\right)}_{B_{E K E}} \underbrace{-\frac{g}{n_{0}} \overline{\rho^{\prime} H_{\rho}^{\prime}}}_{D_{E A P E}}+R_{x_{e d d y}},
\end{gathered}
$$

where

$$
R_{x_{\text {mean }}}=R_{x_{\text {mean }, 0}}+\overline{\rho^{*}} \frac{\partial}{\partial z}\left(\overline{w^{\prime} \rho^{\prime} \frac{g}{n_{0}}}\right), R_{x_{e d d y}}=R_{x_{e d d y, 0}}+\overline{w^{\prime} \rho^{\prime} \frac{g}{n_{0}}} \frac{\partial}{\partial z} \overline{\rho^{*}} .
$$

$B_{M K E}$ is the exchange rate between MKE and MAPE. $R_{\chi_{\text {mean }}}$ and $R_{\chi_{\text {eddy }}}$ do not exist in the APE budgets under quasi-geostrophic assumption (Pedlosky, 1987; von Storch et al., 2012) and these two terms are not the focus of this chapter. Note that the global integral of $B_{E A P E}+B_{M A P E}$ is

$$
\left.\left.\iiint_{V}\left(B_{E A P E}+B_{M A P E}\right) d \nu=\iiint_{V} \nabla_{H} \cdot \overline{\left(\mathbf{u}_{\mathbf{H}}^{\prime} \rho^{\prime} \frac{g}{n_{0}}\right.} \overline{\rho^{*}}\right) d \nu=-\iiint_{V} \frac{\partial}{\partial z} \overline{\left(w^{\prime} \rho^{\prime} \frac{g}{n_{0}}\right.} \overline{\rho^{*}}\right) d \nu
$$

which is a negligible number under quasi-geostrophic assumption. Our calculation using the ECCO2 state estimate confirms this statement. The global integral of $B_{E A P E}+B_{M A P E}$ is $-0.07 \mathrm{TW}$, which is much smaller than the global integral of $B_{E A P E}(0.51 \mathrm{TW})$ and the global integral of $B_{M A P E}(-0.58 \mathrm{TW})$. 


\subsubsection{Determine the standard error}

The standard error $\sigma / \sqrt{N}$ is used in Table 3.2 and Figures 3-7 3-9. This appendix describes how to determine the standard error of a time series $\mathrm{X}$. Assuming $X$ is normally distributed,

$$
\frac{\bar{X}-\mu}{\sigma / \sqrt{N}}
$$

has a Student's t distribution, where $\bar{X}$ represents the sample mean, $\sigma$ represents the sample variance, $\mu$ represents the true mean of $X$ and $\mathrm{N}$ is the number of degrees of freedom. Thus, at roughly $65 \%$ confidence level, the true mean lies in the following interval

$$
\bar{X}-\frac{\sigma}{\sqrt{N}}<\mu<\bar{X}+\frac{\sigma}{\sqrt{N}}
$$

The number of degrees of freedom can be determined from the following formula:

$$
N=M / \max \left[\sum_{n=-N_{0}}^{N_{0}} C(n)\right],
$$

where $M$ is the number of data in the time series and $C(n)$ is the autocorrelation function of $X$, which can be determined from the $x \operatorname{cov}$ command in Matlab. $\sigma / \sqrt{N}$ is the one standard error. The window of \pm 2 standard errors about $\bar{X}$ corresponds to the $95 \%$ confidence interval. 


\section{Chapter 4}

\section{Quantify and interpret striations in a subtropical gyre from a spectral perspective}

\subsection{Introduction}

As the ocean modeling and observation techniques enter the eddy regime, a new feature of the oceanic circulation emerges: the temporal average of some oceanic variables, such as zonal velocity, has banded structures. These banded structures have been identified from eddying numerical models (Cox, 1987; Richards et al., 2006; Nakano and Hasumi, 2005; Galperin et al., 2004), the satellite altimetric data (Maximenko et al., 2005), and the insitu XBT/float data (Maximenko et al., 2008). Previous studies term these features jets or striations. This chapter follows the terminology "striations". As an integral part of oceanic circulation, striations are pervasive in almost all the ocean basins, and they contribute to the transport of heat, tracers, chemicals and biota (e.g. Baldwin et al., 2007; Kamenkovich et al., 2009a). Thus, 
many studies have been done recently about striations. Yet, many aspects about striations are still under debate, such as their amplitude and origins.

Oceanic variability has much larger amplitude than the time-mean circulation and part of the oceanic variability has banded structures; however, it is still unknown how much oceanic variability is associated with striations. Banded structures in the regions away from the Southern Ocean are visible in the temporally averaged eddy field, not in the flow snapshot (Berloff et al., 2011; Thompson, 2010). Thus, many previous studies explore striations by temporally averaging the eddy field over some time period, such as 18 weeks, 4 months, or 3 years (Richards et al., 2006; Nakano and Hasumi, 2005; Maximenko et al., 2005). The amplitude of banded structures generally decreases as the averaging length increases. It is still unclear how long the temporal average should be in order to include all the striations but not other eddies. Exploring this question can help us quantitatively determine the role of striations in eddy energy budgets, tracer transport and mixing process.

Another aspect under debate is the origin of striations. One hypothesis is that they are Rhines jets, which arise from the arrest of inverse cascade by beta effect (Rhines, 1975, 1979). Previous studies show that Rhines jets can form in the barotropic quasi-geostrophic system on a beta plane (Rhines, 1975, 1979), the two-layer quasigeostrophic system on a beta plane with mean vertical shear imposed (Panetta, 1993; Thompson, 2010; Boland et al., 2012), and the three-dimensional primitive equation system (Sayanagi et al., 2008). Schlax and Chelton (2008) proposed that striations could be an artifact of vortex propagation. They found that the temporal average of westward propagating vortices, with statistical characteristics similar to altimeter observations, has striation features, as cyclones (anticyclones) contribute westward (eastward) flow at the northern edge of the track and eastward (westward) flow at the southern edge of the track. We term this hypothesis the "vortex-propagation 
mechanism". Other formation mechanisms include, but are not limited to, stationary Rossby waves (Maximenko et al., 2008), beta plumes (Afanasyev et al., 2011), radiating instabilities of the eastern boundary current (Hristova et al., 2008; Wang et al., 2012), and the nonlinear self-interactions of the linear eigenmodes (Berloff, 2005; Berloff et al., 2009).

The large-scale wind driven flow, which is ignored in the mechanisms summarized above, further complicates the origin and characteristics of striations. Wang (2011) discussed the effect of the double-gyre flow on radiating instabilities. Previous studies found that striations can be generated in two-layer basin models forced by double-gyre winds and their origin is interpreted using concepts, such as Rhines jets, nonlinear rectification of basin modes and Rossby wave instabilities (e.g. Tanaka and Akitomo, 2010; O'Reilly et al., 2012). However, these studies do not explicitly examine the role of the gyre mean flow on the origin and properties of striations.

This chapter does not aim to develop a new theory about the origin of striations. Instead we study and interpret striations in the subtropical gyre in the frequencywavenumber space. Our goals are two-fold: (1) estimate the percentage of the zonal velocity variability in the subtropical gyre region associated with striations from the ECCO2 state estimate and altimetry (Section 4.3); (2) investigate the effect of a subtropical gyre on the origin and properties of striations in an idealized model (Section 4.4). Applications of our results from the two goals are discussed in Section 4.5. 


\subsection{Basic description about striations in ECCO2 model}

We start our study from a visual description of striations in the time-mean zonal velocity field from the ECCO2 model. Readers can refer to Chapter 2 for details about the model. Figure 4-1 shows striations (upper panel) and the gyre flow (lower panel) in the time-mean circulation on a representative isopycnal in the upper North Pacific. Banded structures are pervasive in the domain and their amplitude is large in the Kuroshio Extension, the Central North Pacific (hereafter CNP) and the East North Pacific (hereafter ENP) regions. ${ }^{1}$

\section{Direction}

Striations in the CNP region are zonal, but striations in the southern part of the ENP region tilt southwestward. The non-zonal feature of striations in the ENP region has been identified in previous studies (e.g. Maximenko et al., 2008; Centurioni et al., 2008). However, the mechanisms for the striation direction difference in these two regions are still under debate. We note that the "environment" of striations in these two regions is different: striations in the ENP region are embedded in the non-zonal gyre flow and potential vorticity contours, whereas striations in the CNP region are embedded in the zonal gyre flow (Figure 4-1). Here we propose that the large-scale gyre flow contributes to the non-zonal feature in the ENP region and this hypothesis will be tested using an idealized model in Section 4.4.

We also identify that, in the CNP region and south of $35^{\circ} \mathrm{N}$ of the ENP region, striations roughly align with the eddy trajectories (Figure 4-2). One can get the trajectories of eddies propagating at the time-mean eddy propagation velocity by

\footnotetext{
${ }^{1}$ This chapter focuses on striations in the CNP and ENP regions and those in the corresponding regions in the idealized model, as the gyre-flow in these regions vary slowly spatially and the dynamics are simpler than other regions in the subtropical gyre, such as the western boundary extensions.
} 


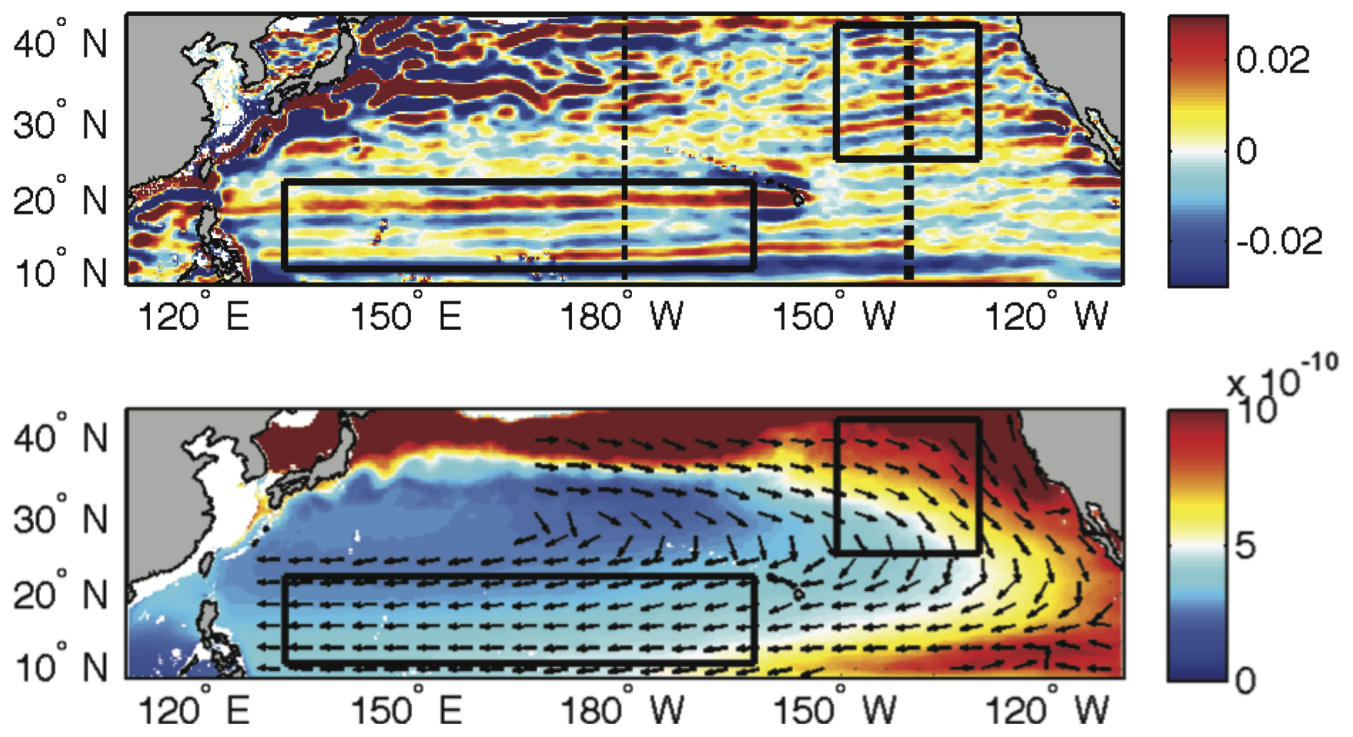

Figure 4-1: The time average of the small-scale zonal velocity over 1992-2007 (upper, $\mathrm{m} / \mathrm{s}$ ), the direction of large-scale time-mean velocity (vectors, lower) and the potential vorticity (color, lower, $\mathrm{m}^{-1} \mathrm{~s}^{-1}$ ) on the isopycnal $1025.6 \mathrm{~kg} / \mathrm{m}^{3}$ from the ECCO2 state estimate. Black boxes denote the Central North Pacific and East North Pacific regions. Here "large-scale" means the $4^{\circ} \times 4^{\circ}$ running average and "small-scale" means the deviation from large-scales. Potential vorticity here is the Ertel potential vorticity, which is approximately $-\rho_{0}^{-1} f d \rho / d z . \rho_{0}$ is the constant reference density and $\rho$ is the in-situ density. Dashed black lines in the upper panel denote the location of the cross sections presented in Figure 4-3.

solving

$$
\frac{d x_{e d d y}(t)}{d t}=C_{x}\left(x_{e d d y}, y_{e d d y}\right), \frac{d y_{e d d y}(t)}{d t}=C_{y}\left(x_{e d d y}, y_{e d d y}\right)
$$

with the initial condition $x_{e d d y}(t=0)=x_{0}$ and $y_{\text {eddy }}(t=0)=y_{0} \cdot x_{e d d y}(t)$ and $y_{e d d y}(t)$ is the eddy position at time t. $x_{0}$ and $y_{0}$ is the initial position. $C_{x}$ and $C_{y}$ are the zonal and meridional components of the 16-year averaged eddy propagation velocity calculated from the correlation method, described in the appendix (Section 4.7.2) and $\mathrm{Fu}$ (2009). Eddies propagate westward in most part of the CNP region, but they propagate in the southwestward direction in most part of the ENP region (Figure 4-2). Consistently, the eddy trajectory tilts southwestward in the ENP region 
and is approximately zonal in the CNP region. However, in both regions, the angle between the eddy propagation direction and the west direction is less than 30 degrees (not shown).

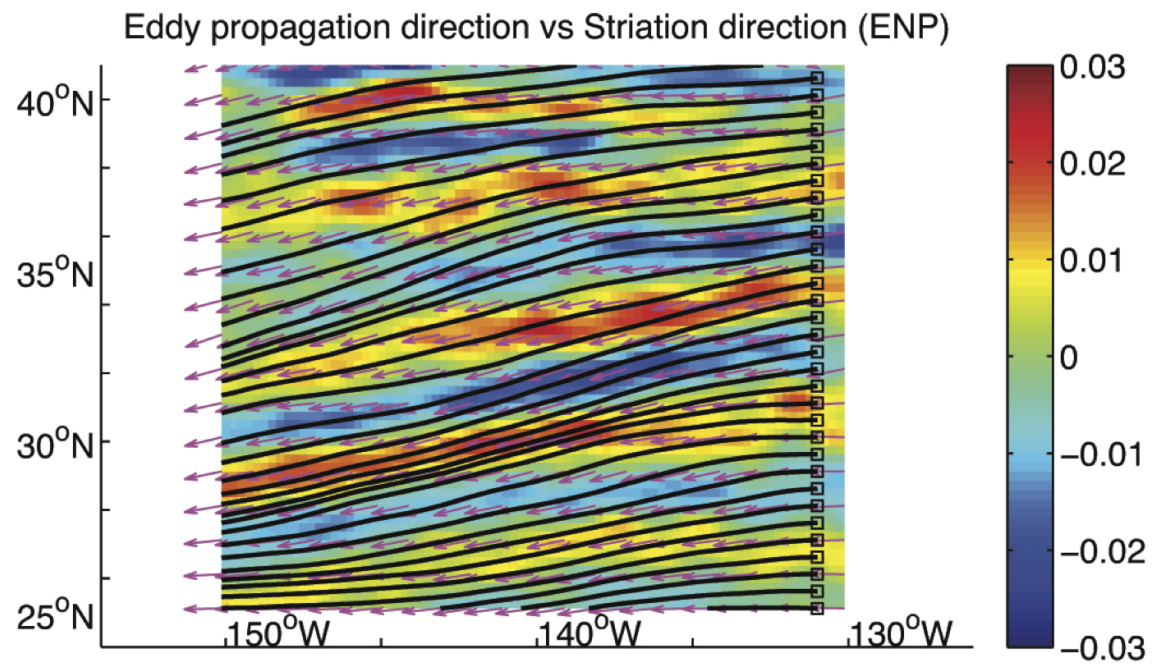

Eddy propagation direction vs Striation direction (CNP)

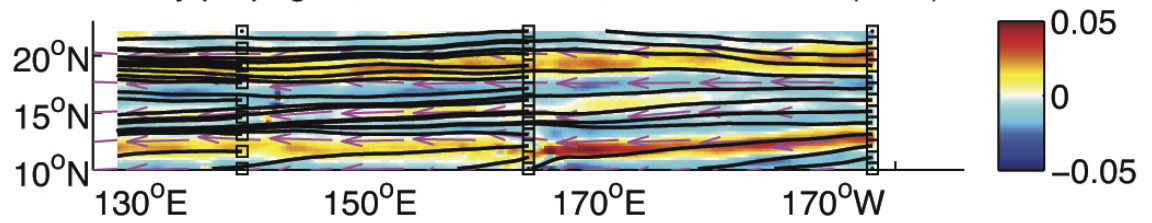

Figure 4-2: The time-mean small-scale zonal velocity (color, $m / s$ ) as shown in Figure $4-1$, the eddy propagation direction (vector), and the eddy trajectories (black line) starting from the black boxes in the ENP (upper) and the CNP (lower) regions at $300 \mathrm{~m}$. The ECCO2 state estimate is used for this diagnosis.

The southward tilt of the eddy trajectories in the ENP region may be partially due to the advection of the southward large-scale gyre flow. Recent observations show that the effect of ocean currents on eddy propagation velocity in the mid- and high latitudes is large $(\mathrm{Fu}, 2009)$. There is quite a large literature about interpreting the propagation speed of vortices and planetary waves using linear theories with/without topography and mean flow (e.g. Tulloch et al., 2009; Killworth et al., 1997). However, the effect of meridional mean flow on the meridional eddy propagation speed in real- 
istic contexts is much less explored to our best knowledge. Using an idealized model in Section 4.4, we will further discuss the effect of the meridional gyre flow on eddy propagation direction, and the consistency between the eddy propagation direction and the striation direction.

\section{Vertical structure}

Figure 4-3 shows representative vertical structures of striations in the two regions. In the upper $1500 \mathrm{~m}$, striations are surface intensified, but their positions and directions have strong vertical coherence. Their amplitude greatly decreases with depth, with the surface amplitude 2 (6) times larger than that at $1500 m$ in the CNP (ENP) cross section. In the upper $1500 \mathrm{~m}$, the meridional width of striations is around 1-2 degrees, whereas narrower striations with the width of around 0.5 degree show up between $1500 \mathrm{~m}$ and $3500 \mathrm{~m}$. The differences of striation characteristics between the upper and deep ocean suggest that the striation dynamics in the upper ocean might be different from that in the deep ocean. The difference could be related to topography, low eddy energy level in the deep ocean and the upper-ocean intensification of the large-scale wind-driven flow. The structures below $3500 \mathrm{~m}$ are not shown, as striations are invisible there. The transport due to eastward striations is approximately the same as the transport due to westward striations in these two regions in the upper $3500 \mathrm{~m}$.

The vertical coherence of the striation position and the decrease of the striation amplitude with depth have also been reported in previous studies (e.g. van Sebille et al., 2011; Richards et al., 2006). This feature suggests that both the barotropic and baroclinic components are non-negligible parts of striations (Kamenkovich et al., 2009b). The amplitude of the barotropic component is $0.4 \mathrm{~cm} / \mathrm{s}(0.3 \mathrm{~cm} / \mathrm{s})$ along the ENP (CNP) cross section. The baroclinic component is surface intensified. Its amplitude in the upper $1000 \mathrm{~m}$ is $0.6 \mathrm{~cm} / \mathrm{s}$ in both cross sections, which is 5 (3) times 

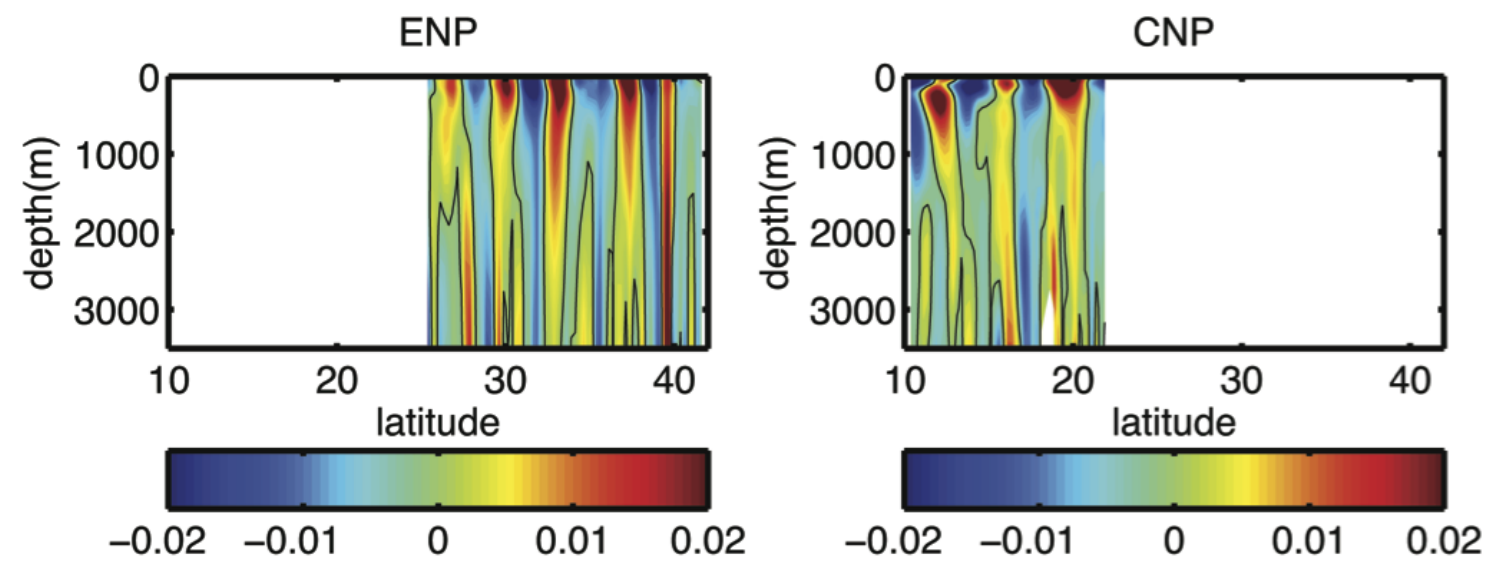

Figure 4-3: Meridional cross sections of the time-mean small-scale zonal velocity $(\mathrm{m} / \mathrm{s})$ along the thick (left panel) and thin (right panel) black dashed lines, indicated in the upper panel of Figure 4-1. These cross sections are representative in the ENP region (left panel) and the CNP region (right panel).

larger than that between 1000-3500 $m$ in the ENP (CNP) cross section.

\subsection{Percentage of eddies associated with striations in ECCO2 model and altimetry}

This section tackles our first question: estimating the percentage of the zonal velocity variability associated with striations. We first introduce a striation definition suitable for our analysis and then present the results.

\subsubsection{Our definition of striations}

As discussed in the introduction and presented in the last section, striations are mostly studied using the time-mean flow field. Depending on the record length and the research goal, many choices of averaging length have been used, such as 18 weeks, 2 years or 8 years (e.g. Maximenko et al., 2005; van Sebille et al., 2011). We find that, 
in the CNP and ENP regions from the ECCO2 state estimate, the amplitude of the time-mean small-scale ${ }^{2}$ zonal velocity at $1000 \mathrm{~m}$ decreases $30 \%$ as one increases the averaging length from one year to the longest available averaging length (16 years). However, it remains unclear whether the amplitude will continue to decrease or level off, if we further increase the averaging length beyond 16 years. It could be that some striation features, such as those associated with topography (e.g. Hawaiian islands) via the beta plume effect, are permanent features.

Here we define striations as the banded structures in the low-frequency motions, because the temporal average essentially captures low-frequency features. This new definition is consistent with the definition in previous literature and will be used in all the remaining analysis about striations in the thesis. It has some advantages: we can use this definition to characterize and interpret striations properties in the frequencywavenumber space, to explore the temporal variability of striations and the energy source of striations, and quantify the contribution of striations to tracer mixing. ${ }^{3}$ Striations based on this definition include bands over a range of wavenumbers. Among these, there is a dominant wavenumber $\left(\vec{k}_{S}\right)$, which occurs at the maximum value in the wavenumber spectrum of low-frequency motions. The striation direction is approximately perpendicular to $\vec{k}_{S}$ and the striation width is roughly $2 \pi /\left|\vec{k}_{S}\right|$. Note that different oceanic variables have different wavenumber spectra; thus, $\vec{k}_{S}$ can be sensitive to the oceanic variable one chooses (e.g. temperature, stream function, zonal velocity).

Since striations are bands in the low-frequency motions, a question arises: what is the separation frequency $\left(\Omega_{S}\right)$ between low-frequency and high-frequency eddies. Using striations in the zonal velocity field as an example, we introduce one pos-

\footnotetext{
2 "Small-scale" is defined in the caption of Figure 4-1.

${ }^{3}$ Utility of this definition in the studies about energetics and mixing is illustrated in Chapter 5.
} 
sible method to determine $\Omega_{S}$. First, we introduce the concept of the normalized wavenumber spectrum of zonal velocity at frequency $\omega_{I}$ :

$$
\left|\tilde{u}\left(k, l, \omega_{I}\right)\right|^{2}=\left|\hat{u}\left(k, l, \omega_{I}\right)\right|^{2} / \max \left(\left|\hat{u}\left(k, l, \omega_{I}\right)\right|^{2}\right),
$$

where $u$ is the zonal velocity, $k$ is zonal wavenumber and $l$ is meridional wavenumber. $\left|\hat{u}\left(k, l, \omega_{I}\right)\right|^{2}$ is the frequency-wavenumber spectrum of zonal velocity in an ocean region and $\max \left(\left|\hat{u}\left(k, l, \omega_{I}\right)\right|^{2}\right)$ is the maximum value of $\left|\hat{u}\left(k, l, \omega_{I}\right)\right|^{2}$ at frequency $\omega_{I}$. Second, we define $\left(k_{n}, l_{n}\right)$ as the wavenumbers where $\left|\tilde{u}\left(k, l, \omega_{I}\right)\right|^{2}$ is larger than 0.2 (an arbitrary choice). The optimum ellipse denotes the smallest ellipse of which all the $\left(k_{n}, l_{n}\right)$ are inside, as illustrated in Figure 4-5. If the optimum ellipse is very narrow, eddy structures are elongated along the minor axis of the ellipse and thus banded structures dominate in the zonal velocity field at frequency $\omega_{I}$ (Figure 4-4). Therefore, here is our criterion: if the ratio between the major and minor axes of the optimum ellipse on $\left|\tilde{u}\left(k, l, \omega_{I}\right)\right|^{2}$ is larger than three (an arbitrary choice), then the optimum ellipse is narrow and $\omega_{I}$ is one of the frequencies with striations. In the zonal velocity spectra in the ocean, the ratio between the major and minor axes of the optimum ellipse generally decreases as the frequency increases. $\Omega_{S}$ is the highest frequency where all the ratios at frequencies lower than $\Omega_{S}$ is larger than three.

However, the above method to obtain $\Omega_{S}$ has some limitations. It does not apply well in the region with large-area of land points, as it is challenging to compute the wavenumber-frequency spectrum in a rectangular area with land points. If only a very small percentage of area in a region is land points, one can pad these land points with values surrounding them and the result is not contaminated much. Quite a few seamounts exist below $1100 \mathrm{~m}$ in the CNP region. Thus, next we only discuss the percentage of variability associated with striations in the upper $1100 \mathrm{~m}$ from the 
(a) Narrow optimum ellipse

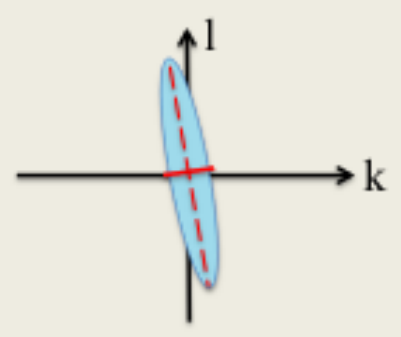

(c) Wide optimum ellipse

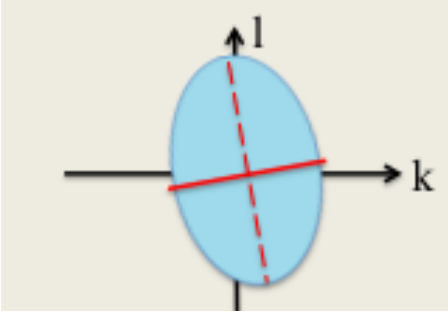

(b) Elongated eddies

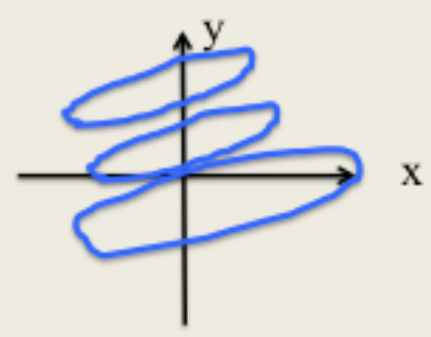

(d) Isotropic eddies

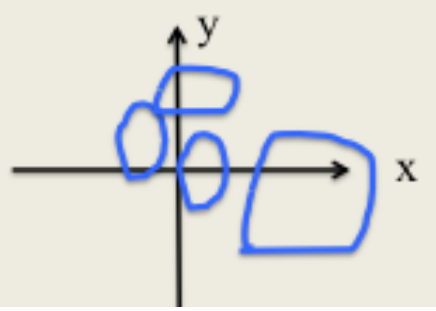

Figure 4-4: Schematics illustrating how we determine the separation frequency $\Omega_{S}$. (a) If the optimum ellipse is very narrow, most variability is along the major axis (dashed red line) and thus eddies are elongated in the direction of the minor axis (solid red line), as illustrated in (b). (c) If the optimum ellipse is wide, the percentage of oceanic variability at all directions are comparable to each other, eddies are more isotropic, as illustrated in (d).

ECCO2 state estimate and the percentage from the altimetric data.

\subsubsection{Results}

As defined above, striations are the part of low-frequency motions inside of these narrow optimum ellipses in the frequency-wavenumber space; thus we can calculate the percentage of zonal velocity variability associated with striations using the following formula

$$
\text { percentage }=\int_{-\Omega_{S}}^{\Omega_{S}} \iint_{D_{k l}(\omega)}|\hat{u}(k, l, \omega)|^{2} d k d l d \omega / \iiint_{V}|\hat{u}(k, l, \omega)|^{2} d k d l d \omega
$$


where $\Omega_{S}$ is the separation frequency obtained using the method described above, $D_{k l}(\omega)$ represents the domain inside of the optimum ellipse in the $k-l$ space at frequency $\omega$, and $V$ represents the entire available three dimensional frequencywavenumer space. One caveat is that the percentage estimate is probably sensitive to the record length available, though how the percentage changes with the record length will not be explored here. One could probably also use this method to estimate the percentage of variability in other oceanic variables (e.g. temperature) associated with striations; however, this has not been done yet.

We choose to use the sea surface height anomaly data from AVISO and the zonal velocity output from the ECCO2 model to estimate the percentage. Specifically, we use the gridded weekly sea surface height anomaly data with the resolution of $1 / 4^{o} \times 1 / 4^{o}$ during the year 1992 2009 (Dibarboure et al., 2009). As to the zonal velocity from the ECCO2 model, it is the 3-day averaged field during the year 1992 $\sim 2007$ and it is interpolated from the cube sphere grids onto the uniform $1 / 4^{o} \times 1 / 4^{o}$ grids.

Now we further illustrate how to obtain the percentage using the altimeter data. A key step is to obtain $\Omega_{S}$ for the surface zonal geostrophic velocity anomaly $\left(u_{g e o}^{\prime}\right)$ :

$$
u_{g e o}^{\prime}=-\frac{g}{f} \frac{\partial}{\partial y} \eta^{\prime}
$$

where $\eta^{\prime}$ is the sea surface height anomaly from AVISO and $f$ is the Coriolis parameter. First, we compute the ratio between the major and minor axes of the optimum ellipse for each frequency. The wavenumber spectrum generally gets more and more isotropic as frequency increases and thus the ratio decreases with frequency. For example, the ratio between the major and minor axes of the optimum ellipse is larger than three for the part of $u_{\text {geo }}^{\prime}$ with periods of $5.5 \sim 16$ years, but it is smaller than 

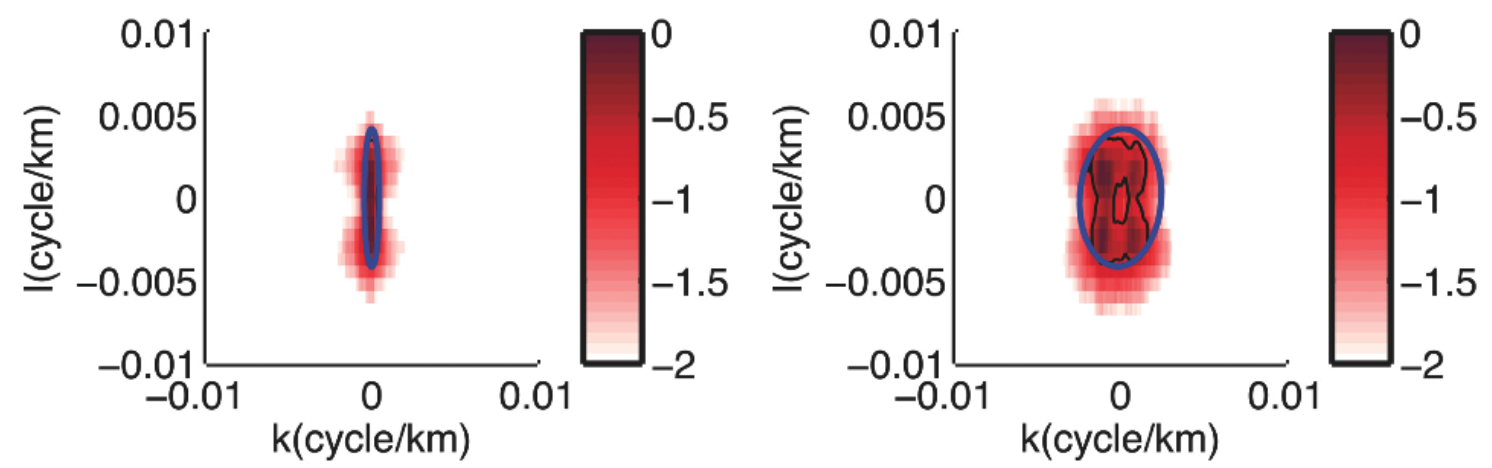

Figure 4-5: The normalized wavenumber spectra for the part of $u_{g e o}^{\prime}$ with periods of 5.5 16 years (left) and 3.7 months (right) in the CNP region. Values of the spectra on the black contours are 0.2. Blue contours denote the optimum ellipses. Colorbars are on the logarithmic scale.

three for the part of $u_{g e o}^{\prime}$ with a period of 3.7 months (Figure 4-5). Inferred from these ratios at all the available frequencies, the separation period $T_{S}\left(1 / \Omega_{S}\right)$ is 8.6 months in the CNP region and 2.7 years in the ENP region. Figures 4-6, 4-7 and 4-8 suggest that this choice of $\Omega_{S}$ is reasonable: the frequency-wavenumber spectrum integrated over frequencies lower than $\Omega_{S}$ is much more anisotropic than that integrated over frequencies higher than $\Omega_{S}$ (Figure 4-6) and banded structures do show up in the part of the eddy field with frequencies lower than $\Omega_{S}$ (Figures 4-7 and 4-8). After $\Omega_{S}$ is obtained, we can estimate the percentage using Eq. 4.1.

Our analysis shows that a non-negligible percentage of zonal velocity variability is associated with striations. Using the method described above, we find that 13\% (23\%) of the variability in surface zonal geostrophic velocity is associated with striations in the ENP (CNP) region. We also estimated the percentage of the zonal velocity variability associated with striations in the upper $1100 \mathrm{~m}$ from the ECCO2 state estimate using the same approach. In the upper $1100 m$ of the CNP and ENP regions, only at 15-35 $\mathrm{m}$ of the ENP region that no frequency passes the criterion and the reason is to be determined. The percentage in the ENP region does not vary 

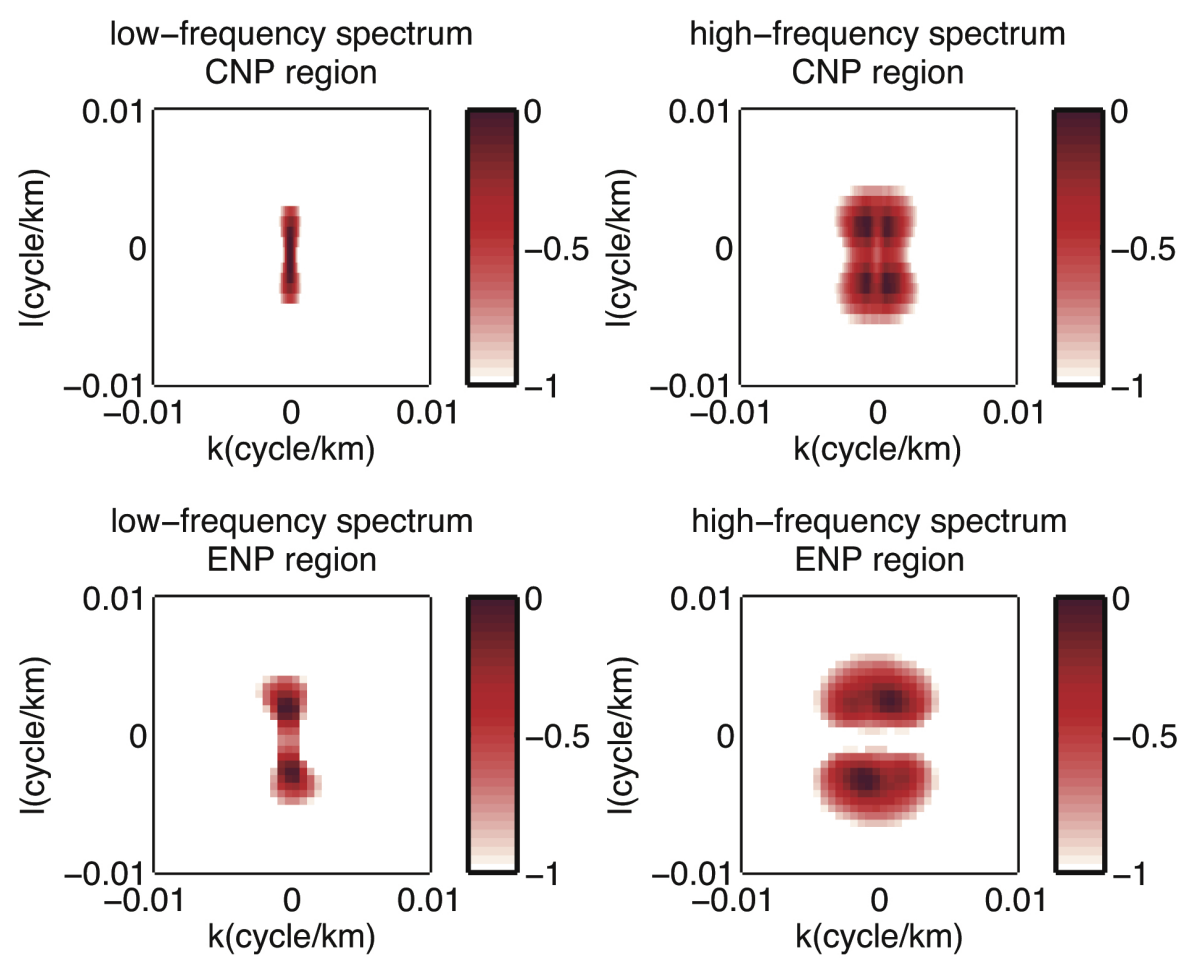

Figure 4-6: Left: normalized wavenumber spectrum of the part of $u_{\text {geo }}^{\prime}$ with frequency lower than $\Omega_{S}$ in the CNP (upper) and ENP regions (lower). Right: normalized wavenumber spectrum of the part of $u_{g e o}^{\prime}$ with frequency higher than $\Omega_{S}$ in the CNP (upper) and ENP regions (lower). Colorbars are on logarithmic scale.

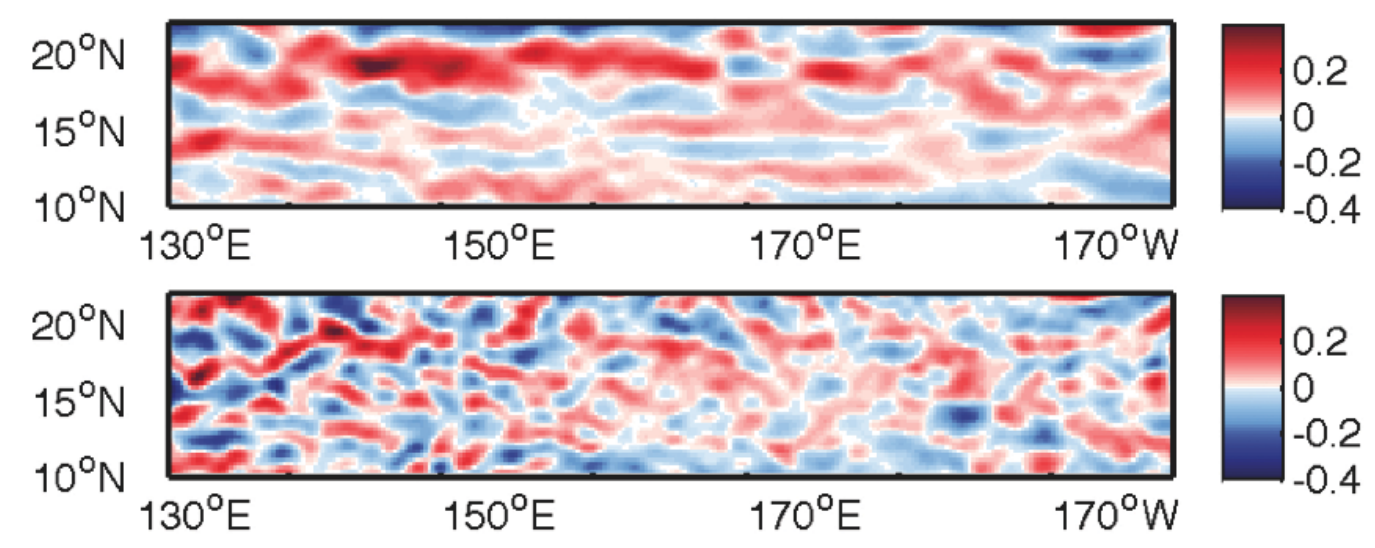

Figure 4-7: Upper (lower) panel shows a snapshot of the part of $u_{g e o}^{\prime}$ in $m / s$ with frequency lower (higher) than $\Omega_{S}$ in the CNP region. 

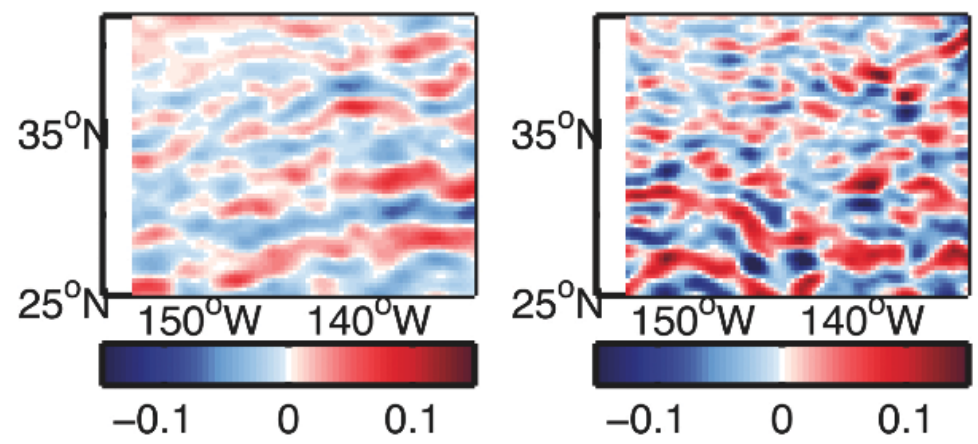

Figure 4-8: Left (right) panel shows a snapshot of the part of $u_{\text {geo }}^{\prime}$ in $\mathrm{m} / \mathrm{s}$ with frequency lower (higher) than $\Omega_{S}$ in the ENP region.

much with depth, but the percentage in the CNP region decreases in the upper 300 $m$ and then levels off (Figure 4-9). The vertically averaged separation period $\left(T_{S}\right)$ in the upper $1100 m$ is 4.1 months (3.2 years) in the CNP (ENP) region. The vertically averaged percentage in the upper $1100 \mathrm{~m}$ is $49 \%$ (14\%) in the CNP (ENP) region. The implications of the surprisingly large percentage are briefly discussed in Section 4.5.1.

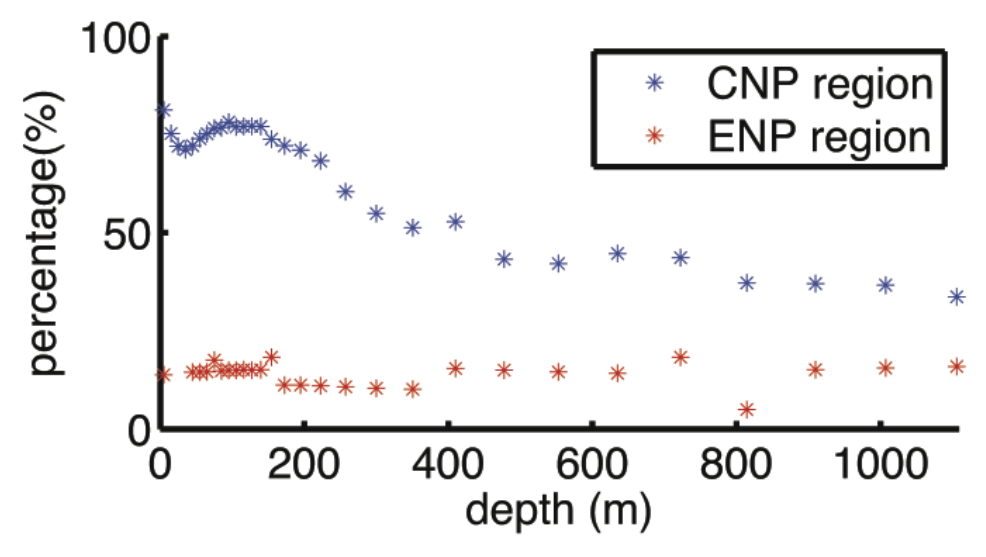

Figure 4-9: The percentage of the zonal velocity variability associated with striations as a function of depth from the ECCO2 state estimate. 


\subsection{Effect of the gyre-flow on idealized striations}

We propose in Section 4.2 that the gyre-flow may contribute to the difference of the striation directions in the CNP and ENP regions. Here we test this hypothesis by studying striations in the gyre-flow using an idealized model. Though greatly simplified, the idealized model can produce striation features qualitatively similar to those in the ECCO2 state estimate.

\subsubsection{Model formulation}

To explicitly examine the role of the gyre flow on the striation direction, we need a model which solves mesoscale motions and at the same time explicitly includes the gyre flow. The multiple-scale expansion method has been used in previous studies about the balance of mesoscale motions (Charney and Flierl, 1981; Pedlosky, 1984). For example, Pedlosky (1984) used this method and developed a stratified quasigeostrophic balance model for mesoscale motions which allows the slow variation of the background stratification due to the gyre-scale flow. Using the same method as Pedlosky (1984), Theiss (2004) formulated a 1.5-layer quasi-geostrophic model with a latitude-dependent deformation radius. Here we also use the method and scaling in Pedlosky (1984) and formulate a 1.5-layer model suitable for our study purpose.

For the convenience of the reader, we provide an informal derivation next. A rigorous derivation is in the appendix (Section 4.7.1).

First, split the velocity into the gyre part $(\mathbf{U})$ and mesoscale part $\left(\mathbf{u}^{\prime}\right)$, and split the layer thickness into the gyre part $(H)$ and mesoscale part $\left(h^{\prime}\right)$. The gyre part of the fields vary on a long scale $(\mathbf{X}, T)$ and the mesoscale part vary on a short scale $(\mathbf{x}, t)$. Assuming $\mathbf{U}$ and $\mathbf{u}^{\prime}$ are of the same order of magnitude as in Pedlosky (1984), the relative vorticity is dominated by the mesoscale part and thus the potential vorticity 
for the 1.5-layer model is

$$
q \approx \frac{f+\zeta^{\prime}}{H+h^{\prime}}
$$

with $f$ denoting the Coriolis parameter and $\zeta^{\prime}$ the relative voticity from mesoscale motions.

Second, assuming that the gyre-scale and mesoscale motions are geostrophic

$$
\mathbf{U}=\widehat{k} \times \frac{1}{f} \nabla_{X}\left(g^{\prime} H\right), \mathbf{u}^{\prime}=\widehat{k} \times \frac{1}{f} \nabla_{x}\left(g^{\prime} h\right),
$$

where $g^{\prime}$ represents the reduced gravity. Since $\mathbf{U} \sim \mathbf{u}^{\prime}$ and $\nabla_{X} \ll \nabla_{x}, h^{\prime} \ll H$. Then, the potential vorticity in Eq. 4.2 can be expanded into the following form

$$
q \approx \frac{f}{H}+\frac{1}{H}\left(\zeta^{\prime}-f \frac{h^{\prime}}{H}\right)
$$

For the second and third term of $q$ in Eq. 4.4 to be on the same order, the short scale should be on the order of deformation radius $\left(\sqrt{g^{\prime} H} / f\right)$.

Then we get the balance equation for potential vorticity defined in Eq. 4.4:

$$
\left(\frac{\partial}{\partial T}+\mathbf{U} \cdot \nabla_{X}\right) \frac{f}{H}+\frac{1}{H}\left[\left(\frac{\partial}{\partial t}+\left(\mathbf{U}+\mathbf{u}^{\prime}\right) \cdot \nabla_{x}\right)\left(\zeta^{\prime}-f \frac{h^{\prime}}{H}\right)+\mathbf{u}^{\prime} \cdot\left(H \nabla_{X} \frac{f}{H}\right)\right]=F_{X}+F_{x},
$$

with $F_{X}$ representing the gyre-scale forcing, $F_{x}$ the mesoscale forcing, and $\nabla$ the horizontal gradient vector $\frac{\partial}{\partial x} \widehat{i}+\frac{\partial}{\partial y} \widehat{j}$.

The model for gyre-scale motions and the model for the mesoscale motions can be obtained from Eq. 4.5. In Eq. 4.5, the terms independent of $(\mathbf{x}, t)$ should balance and thus other terms should also balance. Then we get the model for the gyre-scale motions

$$
\left(\frac{\partial}{\partial T}+\mathbf{U} \cdot \nabla_{X}\right) \frac{f}{H}=F_{X}
$$


and the model for the mesoscale motions

$$
\left[\frac{\partial}{\partial t}+\left(\mathbf{U}+\mathbf{u}^{\prime}\right) \cdot \nabla_{x}\right]\left(\zeta^{\prime}-f \frac{h^{\prime}}{H}\right)+\mathbf{u}^{\prime} \cdot\left(H \nabla_{X} \frac{f}{H}\right)=H F_{x}
$$

Eq. 4.7 is the idealized model we aim to get: the effect of the subtropical gyre is explicitly included in the model through $\mathbf{U}$ and $H$. Compared to the original Rhines jet model in Rhines $(1975,1979)$, which is a barotropic (one-layer) quasi-geostrophic model on a beta plane, our striation model is different in several aspects: the new model is a 1.5-layer model; the background potential vorticity is influenced by the gyre-scale layer thickness $H$ and thus can be non-zonal; potential vorticity is advected by both the mesoscale motions $\left(\mathbf{u}^{\prime}\right)$ and the gyre-scale motions $(\mathbf{U})$.

\subsubsection{Experiment setup}

Here we solve the idealized model to investigate striations. For simplicity purpose, we further assume that the large-scale gyre flow is steady and has no slow temporal variations, such as gyre shifts. However, we do not wish to give the impression that the temporal variability of the gyre flow is not important for striations. Section 4.4.5 discusses more about the limitations and advantages of this idealized model. The numerical model is

$$
\left(\frac{\partial}{\partial t}+U \frac{\partial}{\partial x}+V \frac{\partial}{\partial y}\right) \tilde{q}+J(\psi, \tilde{q})+J(\psi, Q)=\mathcal{F}(x, y, t)-r \cdot \tilde{q}
$$

with $\psi$ denoting the eddy stream function and $\tilde{q}=\nabla^{2} \psi-F_{1} \psi$ denoting potential vorticity from the eddy motions. $J$ is the Jacobian operator satisfying

$$
J(a, b)=\frac{\partial a}{\partial x} \frac{\partial b}{\partial y}-\frac{\partial b}{\partial x} \frac{\partial a}{\partial y}
$$


$U$ and $V$ are the zonal and meridional velocity on the gyre-scale. $F_{1}$ is the squared inverse of the deformation radius. $Q=\beta y+F_{1}(U y-V x)$ represents the gyre-scale potential vorticity. Eddies in this idealized model are embedded in the gyre-scale flow and gyre-scale potential vorticity. $\mathcal{F}(x, y, t)$ is the external forcing. $r$ is the friction coefficient.

The numerical code is a descendant of the two-layer channel model written by G. Flierl and used in several previous studies (e.g. Kaspi and Flierl, 2007; Poulin et al., 2010). The major changes made to the code are adding the spatially-varying gyre flow and reducing it to the 1.5-layer form. The western/eastern boundaries are two walls with no normal flow boundary condition and the model is periodic in the meridional direction. We choose to use the channel model instead of a basin model, as zonal boundary layers do not exist at the northern/southern boundaries of the channel and thus their effects on striations can be excluded. The advection scheme is flux-limiter on a C-grid. $\beta$ is $2 \times 10^{-11} \mathrm{~s}^{-1} \mathrm{~m}^{-1}$ and $r$ is $3.5 \times 10^{-8} \mathrm{~s}^{-1}$. The deformation radius $R d$ is $50 \mathrm{~km}$. The domain size $(8960 \mathrm{~km} \times 5120 \mathrm{~km})$ is approximately the size of the North Pacific. Experiments are carried out at $256 \times 256$ grid points and thus the spatial resolution is roughly half of the deformation radius. The temporal resolution is $1 / 8$ day.

The imposed mean flow $(U, V)$ is a steady double gyre (Figure 4-10) and its corresponding stream function is

$$
\Psi_{I}=-1 /\left(\rho \beta_{0} D\right) \operatorname{curl}(\vec{\tau})(W-x)\left(1-e^{-x / \delta_{S}}\right)
$$

where $\rho$ is density, $\beta_{0}$ is the planetary potential vorticity gradient, $D$ is water depth, $\vec{\tau}$ is the wind stress, $W$ the width of the basin and $\delta_{S}$ the western boundary layer thickness. $\operatorname{curl}(\vec{\tau})$ is a sine function (Figure 4-10), and it has similar magnitude and 
meridional structure to the observed wind stress curl in the North Pacific (Risien and Chelton, 2008).
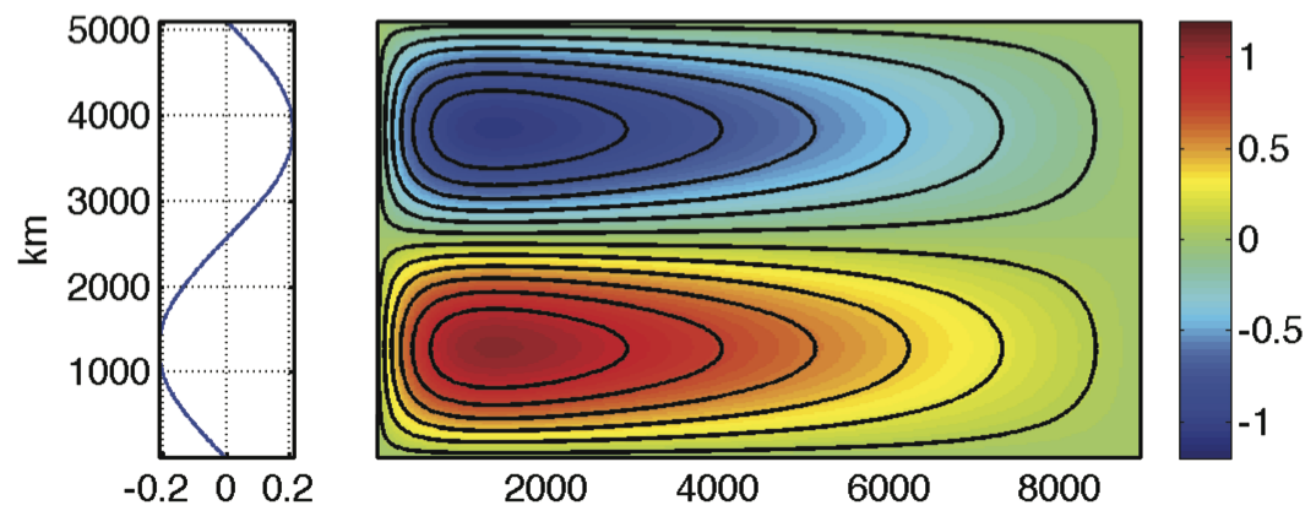

Figure 4-10: Left panel: the wind stress curl $\left(10^{-6} \mathrm{Nm}^{-3}\right)$ that generates the largescale gyre flow on the right panel. Right panel: the stream function $\Psi_{I}$ in $10^{5} \mathrm{~m}^{2} / \mathrm{s}$ from Eq. 4.9. To compute $\Psi_{I}$, I used these parameters: $D=700 m, \beta_{0}=2 \times$ $10^{-11} \mathrm{~s}^{-1} \mathrm{~m}^{-1}, \rho=1025 \mathrm{~kg} / \mathrm{m}^{3}, \delta_{S}=500 \mathrm{~km}$, and $W=8960 \mathrm{~km}$. The $\mathrm{x}$ and $\mathrm{y}$ axes in the right panel are in $\mathrm{km}$.

As in Maltrud and Vallis (1991), we choose to use the random Markovian formulation for the external forcing $\mathcal{F}(x, y, t)$ :

$$
\mathcal{F}_{n}=\sqrt{1-R^{2}} \cdot A_{n}+R \cdot \mathcal{F}_{n-1}
$$

where $\mathcal{F}_{n-1}$ and $\mathcal{F}_{n}$ are respectively the forcing at time steps $n-1$ and $n . R$ is the memory coefficient. The forcing is white noise if $R=0$ and it is steady if $R=1$ (Maltrud and Vallis, 1991). The decorrelation time scale of the observed surface winds in the North Pacific is on the order of a few days (Schlax et al., 2001); thus we choose $R=0.7$, which corresponds to a decorrelation time of 8 times steps (one day). $A_{n}(x, y)$ is random forcing at time step $n$ with a narrow-banded spectrum:

$$
A_{n}=\tilde{A} \cdot F^{-1}\left[e^{-0.01\left(|K|^{2}-K_{0}^{2}\right)^{2}+i \cdot \theta(k, l)}\right] / \max \left(F^{-1}\left[e^{-0.01\left(|K|^{2}-K_{0}^{2}\right)^{2}+i \cdot \theta(k, l)}\right]\right),
$$


where $F^{-1}$ is the inverse Fourier transform operator, $\theta(k, l)$ is random numbers representing the random phase at time step $n$, and $\tilde{A}$ is the forcing amplitude. $K=\sqrt{k^{2}+l^{2}}$, where $k$ is the zonal wavenumber and $l$ is the meridional wavenumber. $K_{0}^{2}=0.0076$ cycle $^{2} /$ grid $^{2}$.

Though the ocean system is nonlinear in most mid- and high latitude regions (Tulloch et al., 2009; Wortham, 2012), linear theories are still useful in interpreting many ocean features, such as eddy length scales and time-mean circulation (Tulloch et al., 2011; Wunsch, 2011). Thus, here we explore both the weakly-forced and stronglyforced solutions of the idealized model. Two experiments are carried out: Exp1 and Exp2. In Exp1, the external forcing has weak amplitude $\left(\tilde{A}=2.7 \times 10^{-12} s^{-2}\right)$; thus, the nonlinear term $J(\psi, \tilde{q})$ is much smaller than the linear terms in Eq. 4.8 and the flow field is approximately linear. This experiment is used to test the validity of the linear analytical solution we formulated next (Eq. 4.14). In Exp2, the external forcing has strong amplitude $\left(\tilde{A}=4.3 \times 10^{-11} \mathrm{~s}^{-2}\right)$. The nonlinear term is of comparable magnitude to the linear terms and this experiment aims to represent the mid-latitude subtropical gyre regions in the ocean. After reaching statistical equilibration, the experiments continue running for roughly 120 years. This 120-year output is used for our analysis. The Central Region and the East Region, shown in Figure 4-11, correspond to the the CNP and ENP regions in the ECCO2 model. The Central Region represents the case with the zonal gyre flow and the East Region represents the case with the non-zonal gyre flow (Figure 4-11). We will focus on studying striations in these two regions due to the reasons stated in Section 4.2.

As the external forcing is narrow-banded, the large values on the wavenumber spectrum at some frequency ranges are not clustered together as those in the realistic context. Thus, the criterion to determine the separation frequency $\Omega_{S}$ in the realistic contexts in the last section does not apply here. Here we choose $\Omega_{S}=1 / 5000$ 
cycle/day. 5000 day is roughly $10 \%$ of the time length of our numerical output and it is on the same order of magnitude as the time scale for long Rossby waves to cross the model basin. Our striation interpretation presented next is not sensitive to the specific value of $\Omega_{S}$. However, the percentage of eddies associated with striations is sensitive to the choice of $\Omega_{S}$. On the other hand, the percentage estimate is less important a problem than that in realistic contexts.

\subsubsection{Striations in Exp1 and the linear theory}

\section{Striations}

Striations exist in Exp1, which is weakly-forced, and are qualitatively similar to those in the ECCO2 state estimate (Figure 4-11). In the linear system, there is negligible energy transfer across spatial and temporal scales in the regions away from the western boundary. Thus, the wavenumber spectrum of the eddy stream function

$\psi(x, y, t)$ has similar structure to that of the external forcing (not shown) and $\psi(x, y, t)$ is also dominated by small scales. The striation width in the zonal velocity field is roughly the same as the dominant spatial scale of the external forcing. Similar to those in the ENP and CNP regions (Figure 4-1), striations here are non-zonal in the East Region and are quasi-zonal in the Central Region.

\section{Interpret striations using the analytical spectrum}

Through analyzing striations in the frequency-wavenumber space, we find that striations are essentially quasi-stationary linear Rossby waves in the linear limit of our idealized model. Assuming the gyre flow $U$ and $V$ are constants in the Central and East Regions and ignoring the nonlinear term $J(\psi, q)$, we can get the analytical 

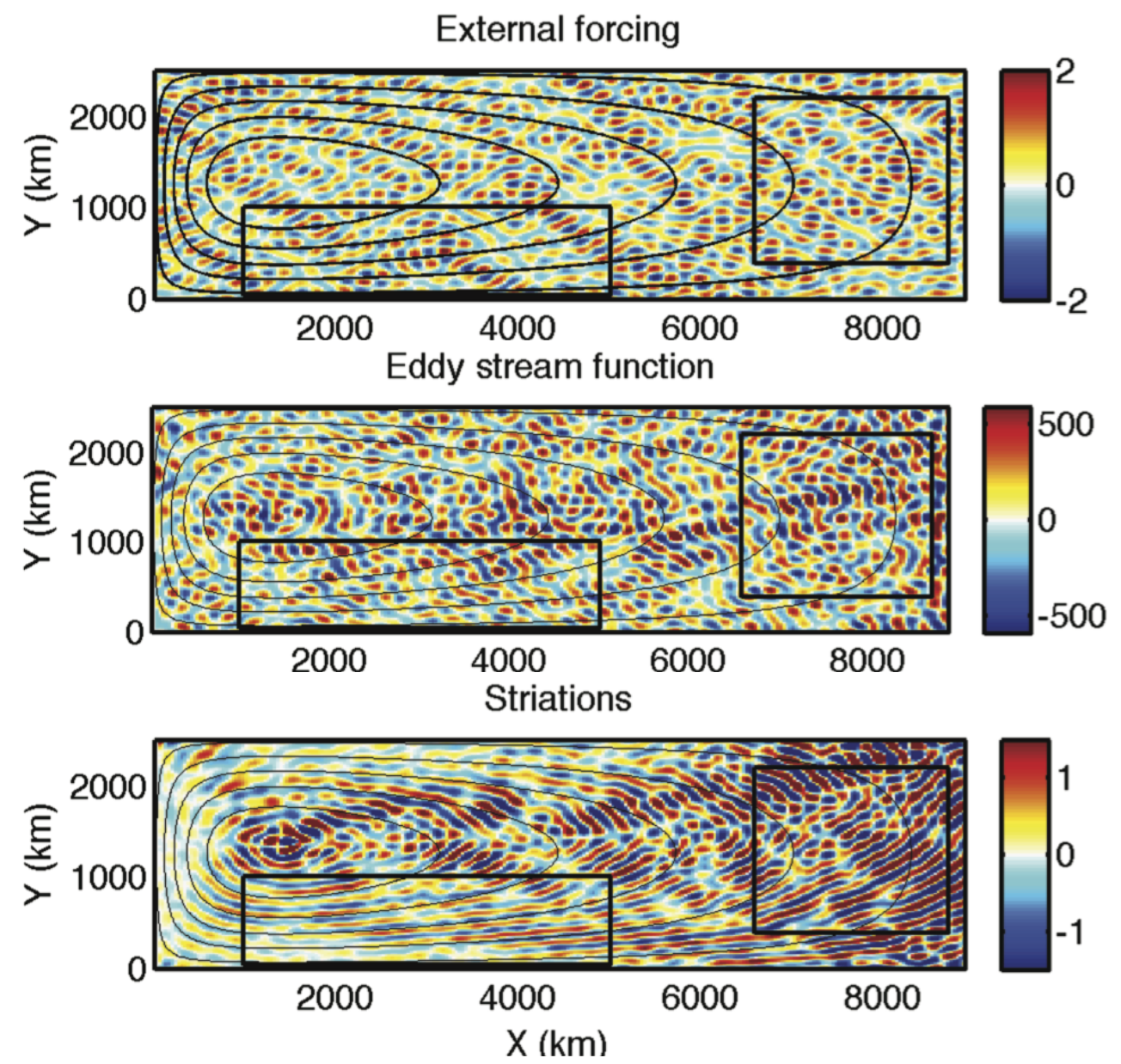

Figure 4-11: Snapshots of the time-dependent external forcing $\mathcal{F}(x, y, t)$ (upper, $10^{-12} \mathrm{~s}^{-2}$ ), eddy stream function $\psi$ (middle, $\mathrm{m}^{2} / \mathrm{s}$ ) and the part of zonal eddy velocity with frequency lower than $\Omega_{S}$ (lower, $10^{-3} \mathrm{~m} / \mathrm{s}$ ) in the subtropical gyre in Exp1. Black boxes denote the Central Region and the East Region, corresponding to the CNP and ENP regions in the North Pacific. Black contours are those of $\Psi_{I}$.

solution to Eq. 4.8. The modal forms of $\psi(x, y, t)$ and $\mathcal{F}(x, y, t)$ are

$$
\psi(x, y, t)=\iiint \hat{\psi}(k, l, \omega) e^{i(k x+l y-\omega t)} d k d l d \omega
$$

and

$$
\mathcal{F}(x, y, t)=\iiint \hat{\mathcal{F}}(k, l, \omega) e^{i(k x+l y-\omega t)} d k d l d \omega
$$


If we substitute Eqs. 4.12 and 4.13 into Eq. 4.8, we obtain

$$
\hat{\psi}(k, l, \omega)=\frac{\hat{\mathcal{F}}(k, l, \omega) / i}{\left(k^{2}+l^{2}+F_{1}\right)\left[\omega-\Omega_{\text {Rossby }}+i \cdot r\right]}
$$

with

$$
\Omega_{\text {Rossby }}=U k+V l-\frac{\beta_{1} k-\beta_{2} l}{k^{2}+l^{2}+F_{1}}
$$

representing the Rossby wave frequency, $k$ the zonal wavenumber, $l$ the meridional wavenumber, and $\omega$ the frequency. $k, l$ and $\omega$ are all real numbers. Thus, the amplitude of $|\hat{\psi}(k, l, \omega)|$ depends on the forcing amplitude $|\hat{\mathcal{F}}(k, l, \omega)|$, the friction coefficient $r$ and $\left|\omega-\Omega_{\text {Rossby }}\right|$. In the inviscid or small-friction case, resonance occurs at the wavenumbers and frequencies satisfying the Rossby wave dispersion relation $\left(\omega=\Omega_{\text {Rossby }}\right)$. As long as the forcing spectrum is smooth near $\Omega_{\text {Rossby }}$, which is generally true in nature, the energy at $\Omega_{\text {Rossby }}$ is the largest and the eddy field can be viewed as a set of linear Rossby waves. Therefore, striations in this case are a set of quasi-stationary linear Rossby waves.

The striation direction is perpendicular to $\vec{k}_{S}$, which refers to the dominant wavenumber of striations as defined in Section 4.3.1. $\vec{k}_{S}$ roughly satisfies

$$
\left|\hat{\psi}\left(k_{S}, l_{S}, 0\right)\right|=\max [|\hat{\psi}(k, l, 0)|]
$$

with

$$
|\hat{\psi}(k, l, 0)|=\frac{|\hat{\mathcal{F}}(k, l, 0)|}{\left(k^{2}+l^{2}+F_{1}\right) \sqrt{\left(0-\Omega_{\text {Rossby }}\right)^{2}}}
$$

in the inviscid case. Numerical solution, shown in Figure 4-12, is consistent with the analytical solution (Eq. 4.14): $|\hat{\psi}(k, l, 0)|$ has large amplitude both on the circle, where $|\hat{\mathcal{F}}(k, l, 0)|$ is large, and on the curve $\Omega_{\text {Rossby }}=0$ in the $\mathrm{k}-\mathrm{l}$ space. The maximum 
value of $|\hat{\psi}(k, l, 0)|$ occurs at the intersection point between the circle and the curve $\Omega_{\text {Rossby }}=0$, as the numerator (denominator) of Eq. 4.14 reaches the maximum (minimum) at this point. Thus, $\vec{k}_{S}$ occurs at this intersection point.

The gyre flow $(U, V)$ influences the location of the intersection point and thus the striation direction through influencing the curve $\Omega_{\text {Rossby }}=0$. In the East Region, the curve $\Omega_{\text {Rossby }}=0$ deviates from $k=0$, as $V \neq 0$; thus $k_{S} \neq 0$ and striations are non-zonal (Figures 4-11 and 4-12). In the Central Region, the curve $\Omega_{\text {Rossby }}=0$ is close to $k=0$, as $V \approx 0$; thus $k_{S} \approx 0$ and striations are quasi-zonal (Figures 4-11 and 4-12). The caveat is that, besides the zero Rossby wave frequency curve, $|\hat{\mathcal{F}}(k, l, \omega)|$ also influences the striation direction (Eq. 4.14).

\subsubsection{Striations in Exp2 and the eddy-propagation mecha- nism}

\section{Striations}

Figure 4-13 shows snapshots of eddies and striations in Exp2, which represents the nonlinear case. Compared to eddies in Exp1 (Figure 4-11), the spatial scale of eddies here is larger due to inverse cascade. In the nonlinear barotropic system on a beta plane, the deformation radius is infinite and Rhines jets are generated (Rhines 1975). The formation of Rhines jets can be suppressed in the nonlinear 1.5layer quasi-geostrophic system because of the finite deformation radius (Okuno and Masuda, 2003). In this experiment, the deformation radius is $50 \mathrm{~km}$, eddies remain roughly isotropic and thus Rhines jets are not relevant to the striation origin here.

Striations also exist in Exp2, but they are wider and more zonal than those in Exp1 (Figure 4-13). The location of the bands is time dependent, and the striation directions in the East and Central Regions are qualitatively similar to those from 

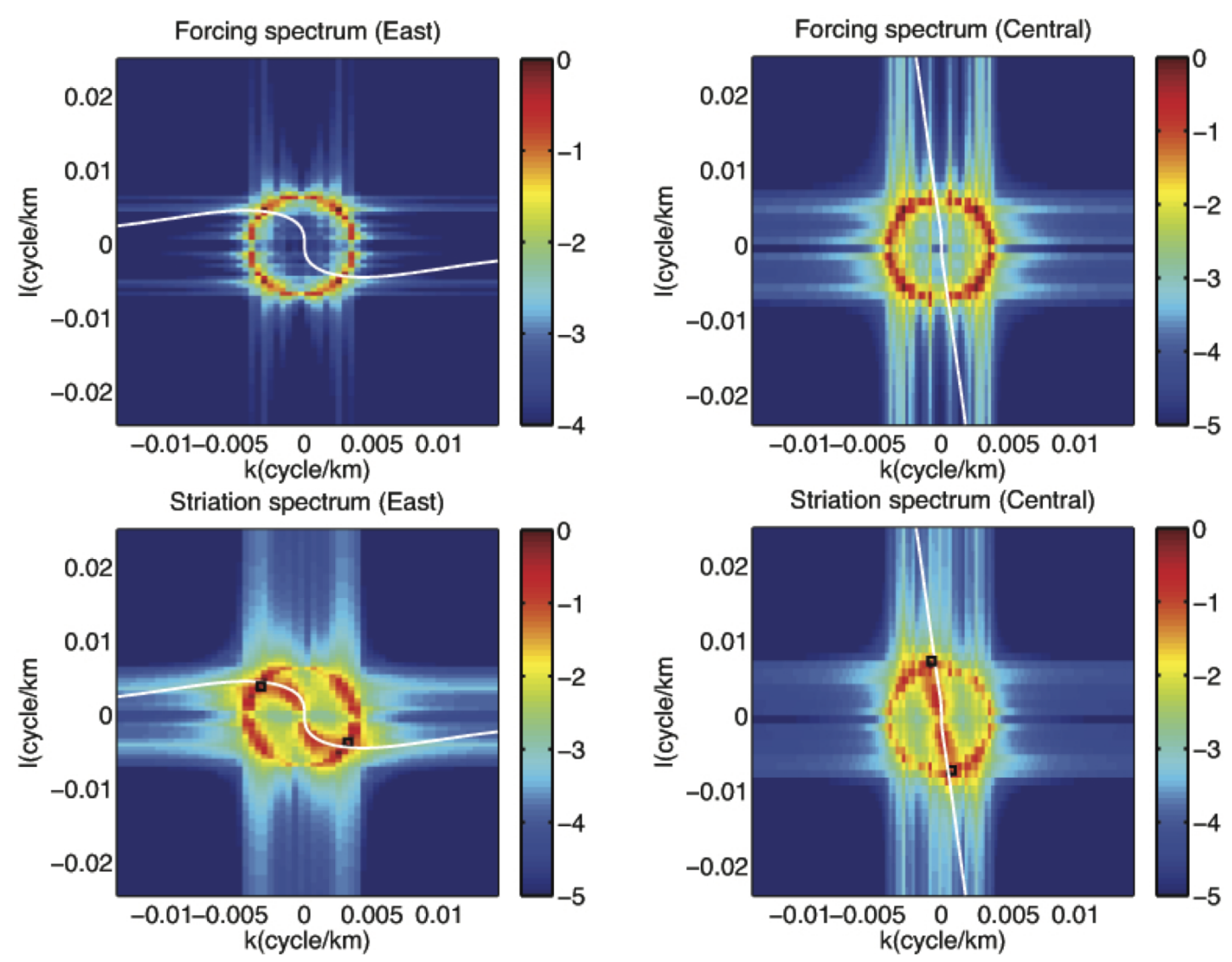

Figure 4-12: Upper panel: normalized wavenumber spectra of the external forcing at zero frequency in the East (left) and Central (right) Regions, that is, $|\hat{\mathcal{F}}(k, l, \omega)|^{2}$ at $\omega=0$; lower panel: the normalized wavenumber spectra of $\psi$ with frequency lower than $\Omega_{S}$ in the East (left) and Central (right) Regions in Exp1. White contours are those of $\Omega_{\text {Rossby }}=0$ and black dots denote $\left(k_{S}, l_{S}\right)$. To calculate the Rossby wave frequency, the imposed gyre flow in the two regions is chosen to be the spatial average of the imposed gyre flow in the two regions. Colorbars are on the logarithmic scale. The difference of the region size and thus the wavenumber resolution between the two regions contribute to the slight difference of the forcing spectrum.

the ECCO2 state estimate (Figure 4-1). We diagnosed the eddy propagation velocity using the correlation method described in the appendix and find that striations roughly align with the eddy propagation direction, as in the ECCO2 state estimate (Figure 4-13). The eddy propagation direction is influenced by the subtropical gyre; for example, the propagation direction in the East Region deviates from the west 


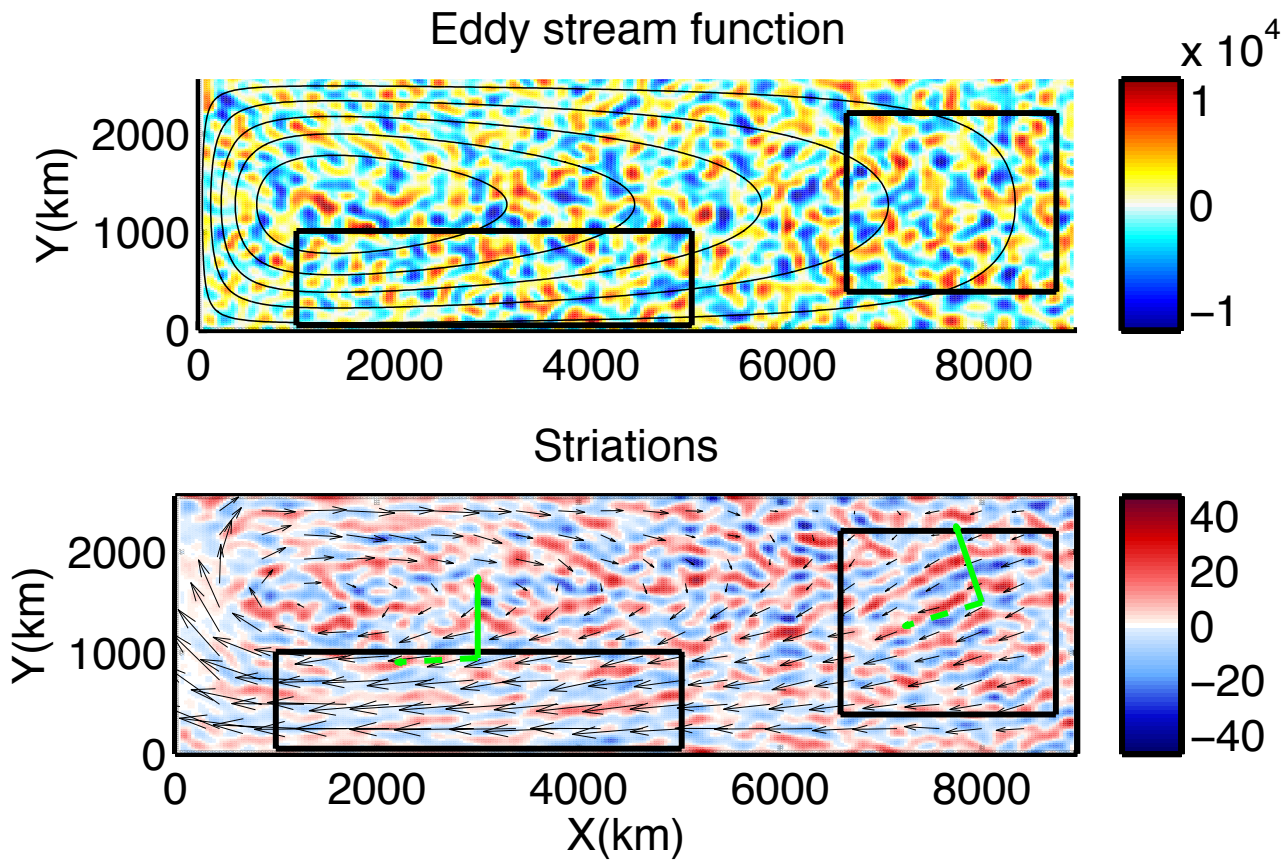

Figure 4-13: Snapshots of the eddy stream function $\psi$ (upper, $\mathrm{m}^{2} / \mathrm{s}$ ) and the part of zonal velocity with frequency lower than $\Omega_{S}$ (lower, $10^{-3} \mathrm{~m} / \mathrm{s}$ ) in Exp2. Black lines denote the subtropical gyre. Black vectors denote the eddy propagation velocity from the correlation method. Dashed green lines denote the direction of the regionally averaged eddy propagation velocity and solid green lines denote the dominant wavenumber identified from the wavenumber spectra of low-frequency zonal velocity in the two regions.

direction due to the southward advection of the gyre flow.

\section{Model spectrum and eddy-propagation mechanism}

To interpret why striations align with the eddy propagation direction, we study striations in the frequency-wavenumber space. As in Exp1, the key is to get the spectrum of the eddy stream function. As one cannot get an exact analytical solution to the nonlinear equation, here we formulate a simple model for the spectrum.

Motivated by the fact that striations align with the eddy propagation direction, we consider an idealized propagating eddy field. Many processes in the ocean can induce eddy variation, such as eddy propagation and eddy-eddy interaction. We assume that 
eddies in the idealized field propagate at a constant velocity

$$
\vec{C}_{e d d y}=C_{x} \vec{i}+C_{y} \vec{j}
$$

with $C_{x}$ denoting the zonal component of the eddy propagation velocity and $C_{y}$ denoting its meridional component. We also assume that the variation of the idealized eddy field due to processes other than eddy propagation occurs on the slow time scale $\epsilon t$, whereas its variation due to eddy propagation occurs on the fast time scale $t . \epsilon$ is a parameter with small magnitude. Supposing we model the idealized eddy field described above as

$$
\psi(x, y, t)=\phi\left(x-C_{x} t, y-C_{y} t, \epsilon t\right)
$$

we obtain

$$
\begin{aligned}
\hat{\psi}(k, l, \omega) & =\iiint \psi(x, y, t) e^{-i(k x+l y-\omega t)} d x d y d t \\
& =\iiint \phi\left(x-C_{x} t, y-C_{y} t, \epsilon t\right) e^{-i(k x+l y-\omega t)} d x d y d t \\
& =\frac{1}{\epsilon} \iiint \phi\left(x^{\prime}, y^{\prime}, \tilde{T}\right) e^{-i\left[k x^{\prime}+l y^{\prime}-\Omega \tilde{T}\right]} d x^{\prime} d y^{\prime} d \tilde{T},
\end{aligned}
$$

with zonal wavenumber $k$, meridional wavenumber $l$ and frequency $\omega, x^{\prime}=x-C_{x} t$, $y^{\prime}=y-C_{y} t, \tilde{T}=\epsilon t$ and $\Omega=\left(\omega-k C_{x}-l C_{y}\right) / \epsilon$. From Eq. 4.17, we obtain the spectrum for the idealized eddy field (i.e. Eq. 4.16):

$$
|\hat{\psi}(k, l, \omega)|^{2}=\frac{1}{\epsilon^{2}}|\hat{\phi}(k, l, \Omega)|^{2}=\frac{1}{\epsilon^{2}}\left|\hat{\phi}\left(k, l,\left(\omega-k C_{x}-l C_{y}\right) / \epsilon\right)\right|^{2}
$$

where

$$
\hat{\phi}(k, l, \Omega)=\iiint \phi(x, y, t) e^{-i(k x+l y-\Omega t)} d x d y d t .
$$


Now we interpret striations in the idealized eddy field using our model spectrum (Eq. 4.18). In the non-propagating case (i.e. $\left|\vec{C}_{\text {eddy }}\right|=0$ ), the model spectrum is reduced to

$$
|\hat{\phi}(k, l, \omega / \epsilon)|^{2} / \epsilon^{2}
$$

Energy tends to cascade to low frequencies (e.g. Arbic et al., 2012) and the spectra in the ocean tend to be red (e.g. Wortham, 2012); therefore it is probably reasonable to assume that the model spectrum for the non-propagating case (Eq. 4.20) has large values in the low-frequency range $\left[-\omega_{0}, \omega_{0}\right]$, not in the high-frequency range $\left[\omega_{0},+\infty\right]$ and $\left[-\infty, \omega_{0}\right]$. Also note that the spectrum of propagating eddies (i.e. Eq. 4.18) is a shift of the spectrum of non-propagating eddies (i.e. Eq. 4.20) in the frequency domain. Thus large values on the spectrum of propagating eddies mostly occur in the range

$$
-\omega_{0} \leqslant\left(\omega-k C_{x}-l C_{y}\right) / \epsilon \leqslant \omega_{0}
$$

that is,

$$
-\epsilon \omega_{0}+\left(k C_{x}+l C_{y}\right) \leqslant \omega \leqslant \epsilon \omega_{0}+\left(k C_{x}+l C_{y}\right) .
$$

Noting that $\epsilon \approx 0$, Eq. 4.21 is reduced to

$$
\omega-k C_{x}-l C_{y} \approx 0
$$

In other words, large values of the frequency-wavenumber spectrum occur on the non-dispersive line: ${ }^{4}$

$$
\omega \approx k C_{x}+l C_{y}=\vec{C}_{e d d y} \cdot \vec{k}=\left|\vec{C}_{e d d y}\right| k_{0}
$$

\footnotetext{
4 "Non-dispersive line" here refers to the straight line, where the large values of the $\omega-k_{0}$ spectrum are concentrated on. Previous studies mostly focus on the non-dispersive lines in the $\omega-k$ spectrum (e.g. Wortham, 2012).
} 
We define $k_{0}$ as the wavenumber along the eddy propagation direction and $l_{0}$ the wavenumber perpendicular to the eddy propagation direction. Since striations are bands in low-frequency motions, the dominant wavenumber of striations $\left(\vec{k}_{S}\right)$ can be derived from Eq. 4.23:

$$
\omega_{S}=\vec{C}_{e d d y} \cdot \vec{k}_{S}=\left|\vec{C}_{e d d y}\right| k_{0 S} \approx 0
$$

where $\omega_{S}$ is the striation frequency and $k_{0 S}$ is the component of $\vec{k}_{S}$ along the eddy propagation direction. Eq. 4.24 further leads to

$$
\vec{C}_{e d d y} \perp \vec{k}_{S} \text { and } k_{0 S}=0
$$

Thus, striations in the idealized eddy field, with constant propagating speed and $\epsilon \approx 0$, align with the eddy-propagation direction.

Here we summarize our striation interpretation based on the spectrum of the idealized eddy field. These idealized eddies propagate at a constant speed $\vec{C}_{e d d y}$, and their variation due to processes other than eddy propagation (e.g. cascades) occurs on a time scale much slower than the eddy propagation time scale (i.e. $\epsilon \approx$ 0 ), that is, the eddy field is quasi-steady in the coordinates moving at the velocity $\vec{C}_{e d d y}$. Large values of the spectrum of this idealized eddy field mostly occur on the non-dispersive line $\omega=\left|\vec{C}_{\text {eddy }}\right| k_{0}$. Thus, though these eddies are composed of motions with a wide range of wavenumbers, only motions with $k_{0} \approx 0$ are lowfrequency motions. Note that $k_{0}$ is the wavenumber along the eddy propagation direction. Therefore, if we further assume the dominant $l_{0}$ for low-frequency motions is finite, low-frequency eddies are dominated by structures elongated along the eddy propagation direction. We provide a diagram to further illustrate this interpretation 
(Figure 4-14). To conclude, from the kinematic and spectral perspective, striations in this idealized scenario align with the eddy-propagation direction and they arise as a temporal averaging effect of eddies propagating at a fixed speed. We term this striation interpretation "eddy-propagation mechanism".

(a) Eddies in the snapshot

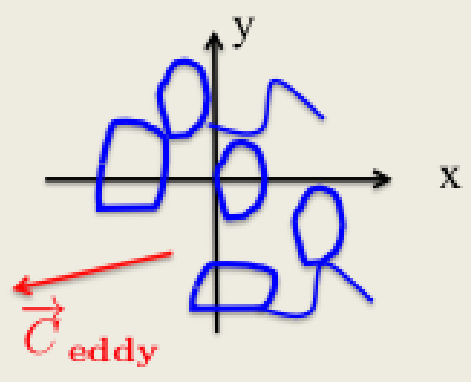

(b) Low frequency eddies

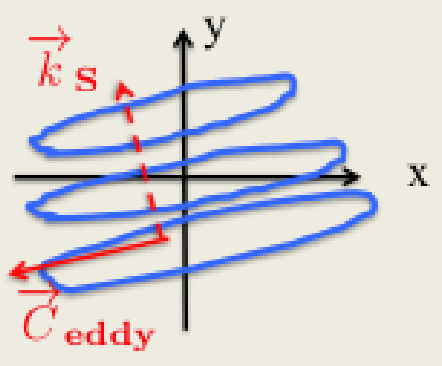

Figure 4-14: This illustrates our striation interpretation based on the model spectrum (Eq. 4.18). (a) In the flow snapshot, eddies include motions over a wide range of wavenumbers and we cannot see banded structures. (b) In the low frequency eddy field, the flow is dominated by motions with wavenumbers perpendicular to the eddy propagation direction; thus elongated structures (striations) along the eddy propagation direction are visible. Blue curves in (a) denote eddies; blue curves in (b) denote striations; solid red arrows denote the eddy propagation direction and the dashed red arrow denotes the wavenumber of striations.

\section{An explicit example of the eddy-propagation mechanism}

The eddy-propagation mechanism is introduced using an abstract function (Eq. 4.16) and its model spectrum (Eq. 4.18). Here we further explain the mechanism and illustrate its relevance using a specific example. Motivated by the ubiquity of vortices in the eddy ${ }^{5}$ field in the ocean (e.g. Chelton et al., 2011), we examine a highly idealized eddy field, which only contains a single Gaussian vortex. Its stream function is

$$
\psi(x, y, t)=A e^{-\frac{\left(t-t_{0}\right)^{2}}{2 T^{2}}} \cdot e^{-\frac{\left[x-x_{0}-\left(t-t_{0}\right) C_{x}\right]^{2}}{2 L^{2}}} \cdot e^{-\frac{\left[y-y_{0}-\left(t-t_{0}\right) C_{y}\right]^{2}}{2 L^{2}}}
$$
$1)$.

${ }^{5}$ Note that eddy in our thesis refer to large-scale and mesoscale subinertial variability (Chapter 
with $A$ denoting its amplitude, $T$ the vortex life time, $L$ the vortex length scale, and $\vec{C}_{e d d y}=C_{x} \vec{i}+C_{y} \vec{j}$ the vortex propagating velocity. $\left(x_{0}, y_{0}\right)$ is its center position at time $t_{0}$. The model spectrum for this vortex is

$$
|\hat{\psi}(k, l, \omega)|^{2}=|A|^{2} e^{-k^{2} L^{2}-l^{2} L^{2}-\left(\omega-k C_{x}-l C_{y}\right)^{2} T^{2}} .
$$

Next we consider a vortex with typical mid-latitude parameters from Chelton et al. (2011). The vortex length scale $L$ is $50 \mathrm{~km}$, the vortex life time $T$ is 32 weeks, and it propagates westward with the speed $5 \mathrm{~cm} / \mathrm{s}$. Note that $T$ is much larger than the vortex propagation time scale $L / \vec{C}_{\text {eddy }}$ (2 weeks); thus $\epsilon$ for this vortex is small, as our idealized eddy field (i.e. Eq. 4.16).

We obtain the two-dimensional spectra for this mid-latitude vortex from Eq. 4.27 and find that the spectra for this vortex have similar properties to the abstract model spectrum (Eq. 4.18). Results are shown in Figure 4-15. The $\omega-k_{0}$ spectrum has large values on the non-dispersive line. As $\omega \rightarrow 0, k_{0}$ for the large values on the spectrum approaches zero, but $l_{0}$ for most large values on the spectrum remains finite (Figure 4-15). Consistently, the wavenumber spectrum for the zero frequency motions has a shape of a narrow optimum ellipse with its minor axis along the $k_{0}$ direction. Thus, the low-frequency component of this propagating single vortex is elongated along the $k_{0}$ direction, as shown in Figure 4-16.

One can extend the above example into a more general case with multiple random vortices. The multiple-vortex case is consistent with the scenario presented in Schlax and Chelton (2008). They found that the temporal average of westward propagating vortices has zonal bands and concluded that striations can be eddy artifacts. Both our eddy-propagation mechanism and their scenario suggest that the temporal average of propagating motions has banded structures. On the other hand, our work is different 

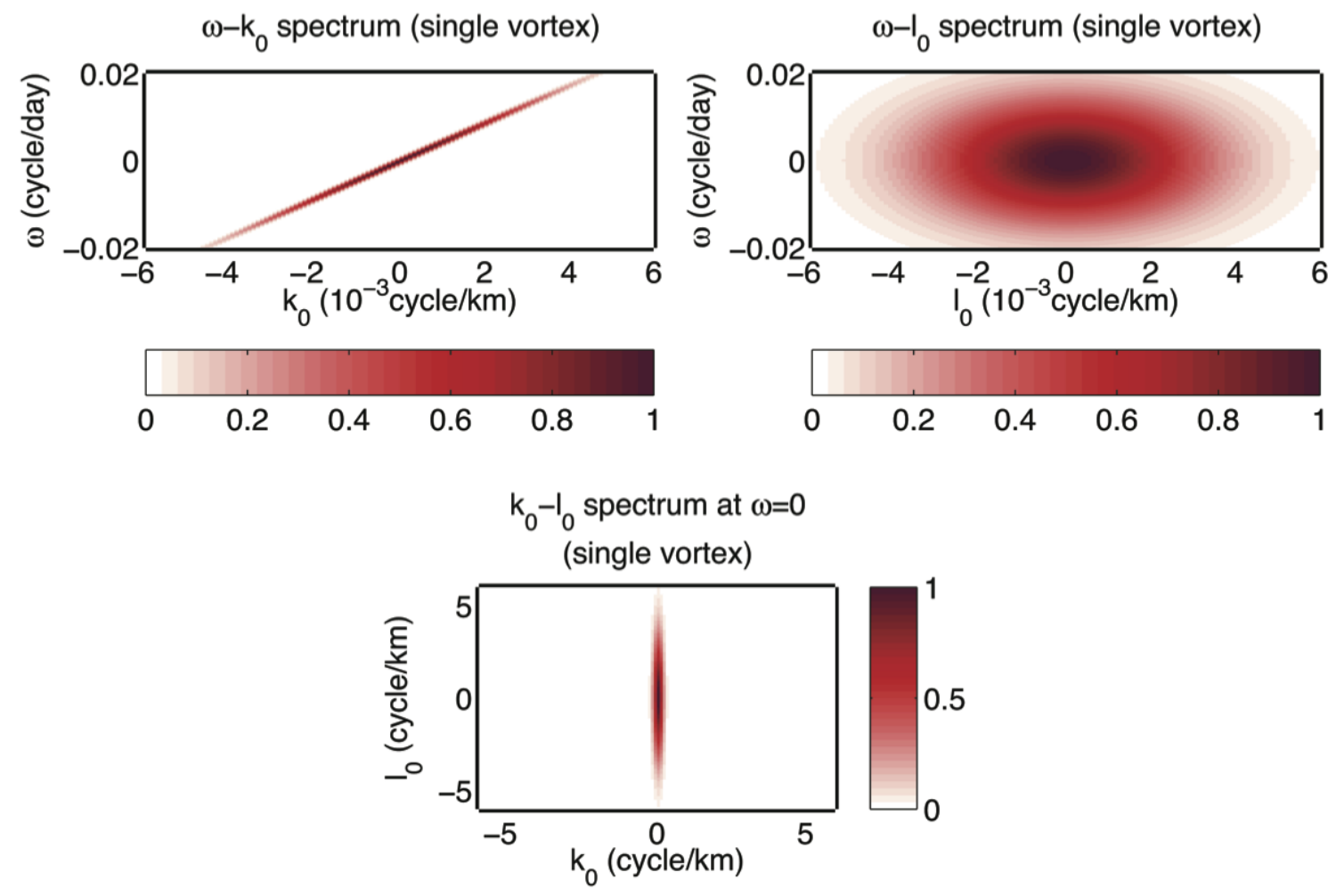

Figure 4-15: Upper panel: normalized $\omega-k_{0}$ spectrum and $\omega-l_{0}$ spectrum of $\psi$ for a single vortex; lower panel: normalized $k_{0}-l_{0}$ spectrum for the zero frequency component of $\psi$ of the single vortex. $k_{0}\left(l_{0}\right)$ is the wavenumber along (perpendicular to) the eddy propagation direction, which is zonal in this case.

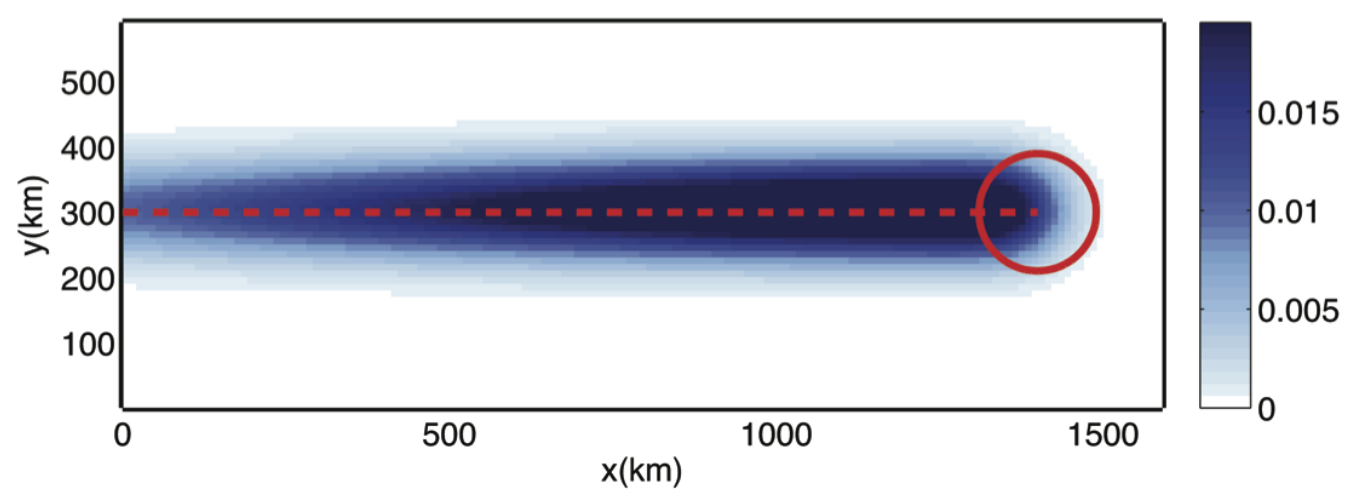

Figure 4-16: The temporal average of the normalized stream function of the single vortex $(\psi / A)$ over 1000 days starting from $t=t_{0}$. The red circle denotes the initial position of the vortex and the red dashed line is the vortex trajectory. 
from their work in some aspects: (1) we provide an interpretation of these bands using model spectrum (Eq. 4.18); (2) we find that $\epsilon \approx 0$ is a prerequisite for the existence of these banded structures in the low-frequency eddy field; (3) our striation definition in Section 4.3 suggests that these bands are part of the propagating eddy field. More discussion about the relation between our work and their work is in Section 4.4.5.

\section{Relevance of the eddy-propagation mechanism to Exp2}

The striation interpretation discussed above is relevant to Exp2. As the model spectrum of the idealized propagating eddies (Eq. 4.18) predicts, striations in Exp2 align with eddy propagation direction (Figure 4-13) and the spectrum indeed has most energy along the non-dispersive line in the $\omega-k_{0}$ space (Figure 4-17). As $\omega \rightarrow 0$,
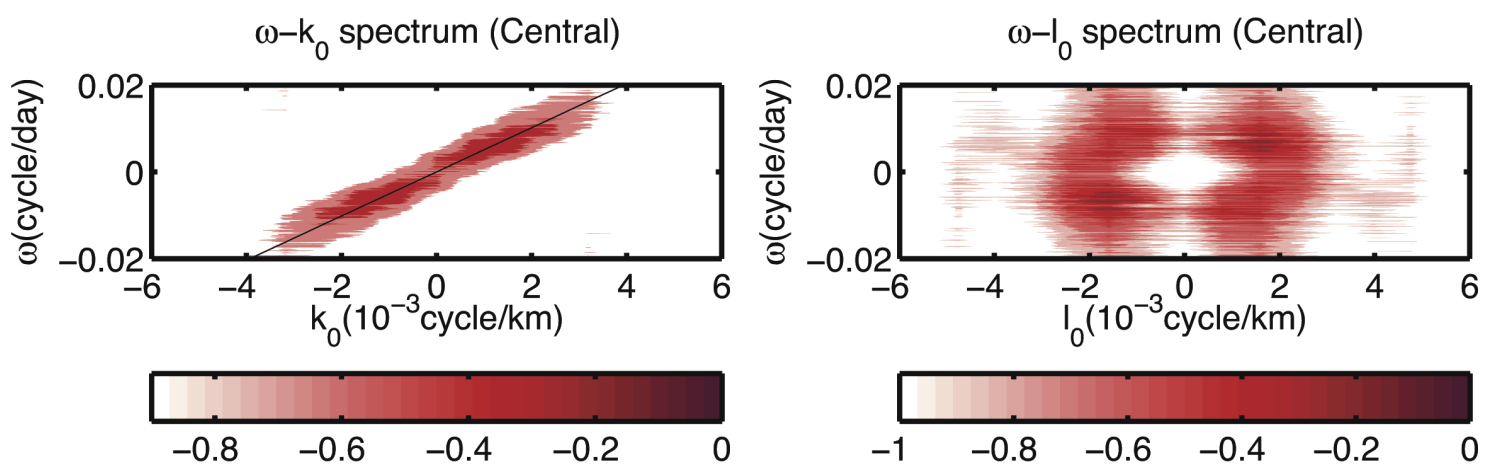

$\omega-\mathrm{k}_{0}$ spectrum (East)

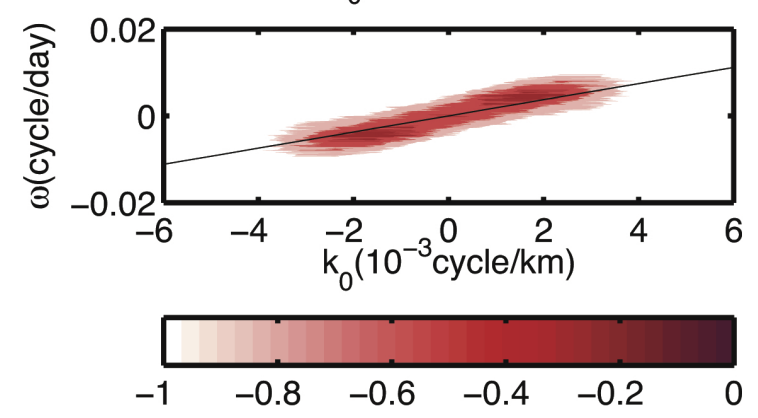

$\omega-I_{0}$ spectrum (East)

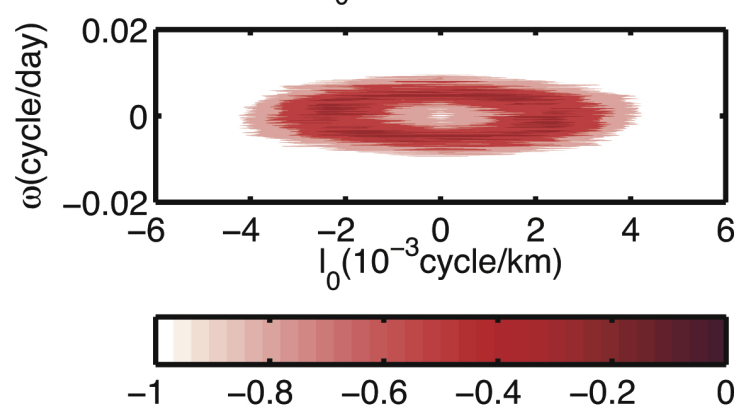

Figure 4-17: Normalized $\omega-k_{0}$ spectrum and $\omega-l_{0}$ spectrum of $\psi$ in the Central Region (upper) and East Region (lower). Here $k_{0}\left(l_{0}\right)$ is the wavenumber along (perpendicular to) the direction of the regionally averaged eddy propagation velocity. The black line is $\omega=\sqrt{C_{x}^{2}+C_{y}^{2}} k_{0}$ in the corresponding region. 
$k_{0} \rightarrow 0$ and $l_{0}$ is finite; thus low-frequency eddies are elongated in the $k_{0}$ direction. Note that $k_{0}$ is the wavenumber along the eddy-propagation direction and $l_{0}$ is the wavenumber perpendicular to the eddy-propagation direction. The non-dispersive line has also been identified by Early et al. (2011) in the zonal wavenumber-frequency spectrum of sea surface height in a nonlinear 1.5-layer model seeded with many eddies.

Figure 4-18 illustrates how the imposed subtropical gyre influences the striations in Exp2. The energy source of eddies is small-scale random potential vorticity forcing and eddies have energy over a wide range of spatial scales as a result of eddy-eddy interaction. Striations exist in both the Central and East Regions, align with the eddy propagation direction, and arise as a temporal averaging effect of eddies propagating in the fixed direction. Eddies are advected by the subtropical gyre and therefore propagate southwestward (westward) in the East (Central) Region. Thus, striations are non-zonal (zonal) in the East (Central) Region. However, more studies are needed to quantitatively determine the role of the gyre flow on the eddy-propagation velocity in both the idealized model and realistic contexts. This is relevant to the open question discussed in Wortham (2012): what sets the slope of the non-dispersive line (dominant phase speed)?

\subsubsection{Discussion}

Here we discuss the limitations and advantages of the idealized model, and point out some remaining challenging puzzles relevant to the above interpretations. The discussion here is incomplete and any further studies will be very useful.

\section{Limitations and advantages of the model}

The idealized model is limited in many aspects. The radiating instabilty of the eastern boundary current can induce tilted banded structures in the flow snapshot 


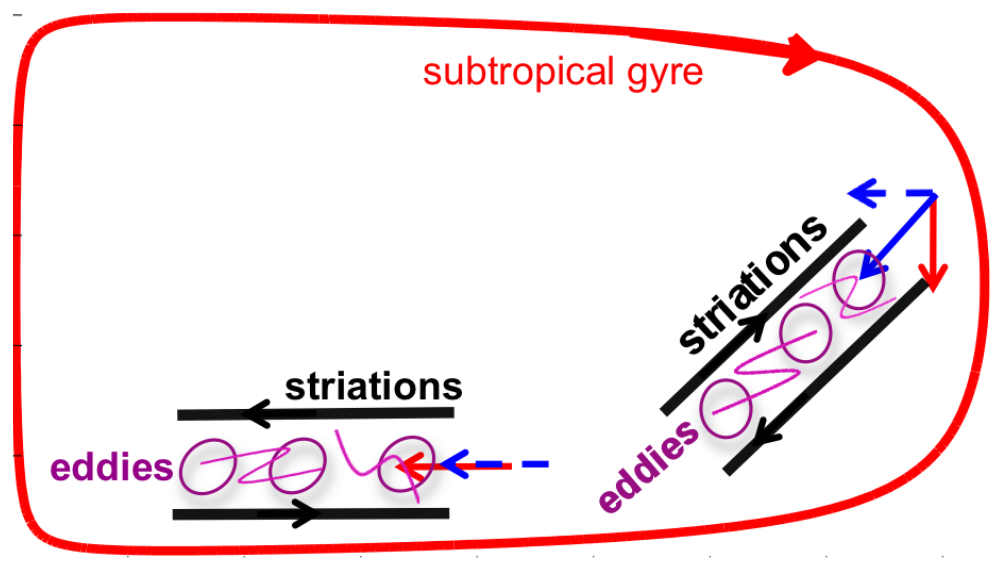

Figure 4-18: A diagram illustrates the eddy-propagation mechanism and the effect of the subtropical gyre. Magenta circles and curves denote the eddy field. Red arrows denote the direction of the gyre flow, dashed blue arrows denote the eddy propagation direction if there is no subtropical gyre and the solid blue arrow denotes the eddy propagation direction if there is a subtropical gyre.

in the ENP region and the localized wind stress curl forcing at the Hawaiian Island can induce the zonal currents in the north CNP region via the beta plume effect (Wang et al., 2012; Stommel et al., 1958; Jia et al., 2011). However, these processes do not exist in the idealized model. Also, this idealized model only has one equivalent barotropic mode, in which the lower layer is quiescent, but striations have both barotropic and baroclinic components. The analysis of the ECCO2 model output and the mooring data suggests that both the first baroclinic mode and the barotropic mode contain significant amount of energy and these two modes are coupled (Wunsch, 1997; Wortham, 2012). Furthermore, baroclinic instability process and eddies' feedback on the gyre flow are not included. We choose to ignore these additional dynamical factors in oder to isolate and focus on the gyre effect. However, we do not intend to claim that these factors are not important for the striation dynamics.

On the other hand, it is easier to identify the physics using a simple model, as it focuses on the limited key factors (e.g. mean flow's impact on striations). The 1.5layer model has been widely used by the community to explore eddy characteristics 
and eddy mixing (e.g. Early et al., 2011; Cushman-Roisin et al., 1990; McWilliams and Flierl, 1979; Jacob et al., 2002; Radko and Stern, 1999; Klocher et al., 2012a). Though greatly simplified, the transport properties/zonal propagation speeds of the nonlinear vortices and the non-dispersive line in the $\omega-k$ spectrum in the nonlinear 1.5-layer model resemble those from the altimetry (Early et al., 2011). The characteristics of striations in our 1.5-layer model, such as the direction, are qualitatively similar to those in the double-gyre from idealized two-layer basin models (e.g. O'Reilly et al., 2012) and to those in the ENP and CNP regions in the ECCO2 model (Figures 4-1 and 4-2). Thus, the 1.5-layer model is a useful tool for the proposed problem as a simple starting point.

\section{Wave mechanism vs. eddy-propagation mechanism}

Analysis in Section 4.4.3 and Section 4.4.4 suggests two striation interpretations: quasi-stationary linear Rossby waves or eddy-propagation mechanism. However, we do not intend to claim that striations from the eddy-propagation mechanism cannot behave like waves. Here are the reasons. At the ocean surface, the eddy field is dominated by waves equatorward of $30^{\circ}$ (Tulloch et al., 2009), but non-dispersive lines on the spectra also exist in some regions equatorward of $30^{\circ}$ (Wortham, 2012). This suggests that striations from the eddy-propagation mechanism might behave like waves in some oceanic regions. Thus, the two striation interpretations (wave mechanism and eddy-propagation mechanism) are probably not completely separable.

Previous studies suggest that, in the nonlinear 1.5-layer quasi-geostrophic system, the parameter $\tilde{U} F_{1} /|\nabla Q|$ may be useful in assessing whether part of the eddy field behaves like waves. $F_{1}$ and $Q$ are defined in Section 4.4 .2 and $\tilde{U}$ is the eddy velocity scale. Penny et al. (2010) provide a more detailed derivation and interpretation than our review below, which readers can refer to (they are not the original source for this parameter though). In a nonlinear barotropic quasi-geostrophic system on a beta 
plane, the deformation radius is infinite and the separation wavenumber between wave-like and turbulence-like motions is

$$
k_{R}=\sqrt{\frac{\beta}{\tilde{U}}|\cos \alpha|},
$$

with $\alpha$ denoting the angle between the wave propagation direction and the east, and $\beta$ denoting the planetary potential density gradient. Motions with wavenumbers smaller than $k_{R}$ behave like waves (Rhines, 1975; Penny et al., 2010). In the nonlinear system with finite deformation radius and the mean gyre-scale flow, as in our idealized model, the separation wavenumber is $k_{C}$, which is a modified version of $k_{R}$ :

$$
k_{C}=\sqrt{\frac{|\nabla Q|}{\tilde{U}}|\cos \alpha|-F_{1}}
$$

(e.g. Okuno and Masuda, 2003). If $\tilde{U} F_{1} /|\nabla Q|>1, k_{C}$ is imaginary and eddy motions behave like turbulence in the entire wavenumber space; if $\tilde{U} F_{1} /|\nabla Q|<1, k_{C}$ is real and eddy motions with wavenumbers smaller than $k_{C}$ is wave-like. This parameter has been used to assess the nonlinearity of vortices (e.g. Chelton et al., 2011) and to examine storms and vortices in gas planetary atmospheres (e.g. Penny et al., 2010; Theiss, 2006). Using this argument, Tulloch et al. (2009) found that the eddy field is dominated by waves equatorward of $30^{\circ}$ from the altimetric observations.

Though the parameter $\tilde{U} F_{1} /|\nabla Q|$ has been widely applied in various contexts, to our best knowledge, few studies exist rigorously testing the validity of using this parameter to separate the wave motions and turbulence motions. This separation issue is still an ongoing research topic of the oceanic community. Thus, it remains challenging to fully clarify the relation between the wave mechanism and the eddy-propagation mechanism about striations in the 1.5-layer model in a solid and convincing way. 


\section{Comparison with the vortex propagation mechanism}

Schlax and Chelton (2008) found that the temporal average of vortices propagating in a fixed direction can produce banded features along the vortex propagation direction. The vortex example in Section 4.4.4 shows how to interpret bands in their work using the spectrum argument. Striations in their work are essentially the lowfrequency component of these propagating vortices; while in our Exp2, striations are essentially the low-frequency component of the propagating eddies. Note that the eddy field in the ocean includes both vortices and the background field, though more than $40 \%$ of eddy kinetic energy is in vortices with life time longer than 4 weeks (Chelton et al., 2011). A natural question is whether the low frequency part of the background field has banded structures. And if it does, how much is their contribution? However, to our best knowledge, theories about the contribution of the background field to the spectrum shape do not exist. The separation of the eddy field into the vortex part and the background part is not trivial. Also the factors controlling the ratio between energy in the background field and energy in vortices are rarely explored, though there are explorations about the emergence and disappearance of coherent structures (e.g. Maltrud and Vallis, 1991; Polvani et al., 1994). Studies in this aspect are useful in further understanding striations' origin.

\subsection{Applications}

Here we discuss some applications of our results in this chapter. First, the large percentage of zonal velocity variability is associated with striations; thus, striations may have non-negligible contributions to tracer transport, mixing and the energy cycle. These contributions can probably be quantified using our striation definition in Section 4.3.1 and our diagnostic frameworks in the next chapter. Second, we infer 
that the eddy-propagation mechanism may be relevant to realistic striations in the interior ocean between a few degrees off the equator and 45 degrees.

\subsubsection{Tracer transport and energy pathway}

The transport of tracers, such as temperature, salinity, pollutant, sediments and larvae, is important to climate variability, coastal environment, pollutant pathway prediction etc. Many studies exist about the contribution of eddies and the mean flow to tracer transport and eddy diffusivity (e.g. Klocher et al., 2012a; Rypina et al., 2012; Shuckburgh et al., 2009). Striations are coherent in the quasi-zonal direction and can cause shear dispersion and thus anisotropic transport. Previous studies found that the tracer transport in the ocean is indeed anisotropic (e.g. Kamenkovich et al., 2009a; Rypina et al., 2012). However, the contribution of striations in the time-mean

circulation to the anisotropic transport is small in the North Atlantic (Kamenkovich et al., 2009a).

We suspect the contribution of striations in the eddy field to the anisotropic transport can be significant, as striations account for a non-negligible percentage of the zonal velocity variability in the ENP and CNP regions. In analogy, the role of striations in the global energy budget is also likely to be important. Our inferences about the significant consequences of striations in the aspect of energetics and tracer spreading are to be tested. Using the criterion presented in Section 4.3.1, one can separate striations from high-frequency eddies and then quantitatively estimate the contribution of total striations to tracer transport and the energy pathway. Chapter 5 moves forward in this direction by presenting and testing the diagnostic frameworks about the role of striations in tracer mixing and energy budgets. 


\subsubsection{Interpret striations in ECCO2 model}

\section{On the applicability of eddy-propagation mechanism}

One can identify the regions where the eddy-propagation mechanism may be relevant from the spectrum in observations and the ECCO2 model. If the non-dispersive line exists in the spectrum in an ocean region, the eddy-propagation mechanism proposed in Exp2 is probably relevant there (Section 4.4.4). Figure 4-19 summarizes the regions where the frequency-zonal wavenumber spectra of sea surface height $(\omega-k$ spectrum) have been presented in previous studies (e.g. Ferrari and Wunsch, 2010; Wortham, 2012). Non-dispersive lines exist in the solid black boxes and do not exist along the white line and the dashed black boxes.

The eddy-propagation mechanism may be relevant in the ocean interior between a few degrees off the equator and 45 degrees off the equator, as Wortham (2012) proposed that the frequency-zonal wavenumber spectra has non-dispersive lines in these regions, through analyzing the sea surface height data from the altimetry and the ECCO2 model. The CNP and ENP regions are within this range. The rough consistency between the eddy trajectories and the striation direction in the CNP region and the southern part of the ENP region further suggests the relevance of the eddy propagation mechanism there (Figure 4-2).

The eddy-propagation mechanism probably does not apply in the western boundary current and its extension regions, the subpolar gyres, and the Southern Ocean. This is because the non-dispersive lines in the $\omega-k$ spectra are absent in these regions (Wortham, 2012). However, observations show that striations also exist in these regions (Figure 1 in Maximenko et al., 2008). A prerequisite for our mechanism is that, in a domain several times larger than the striation width, there is a dominant eddy propagation direction and the eddy propagation speed should be roughly 


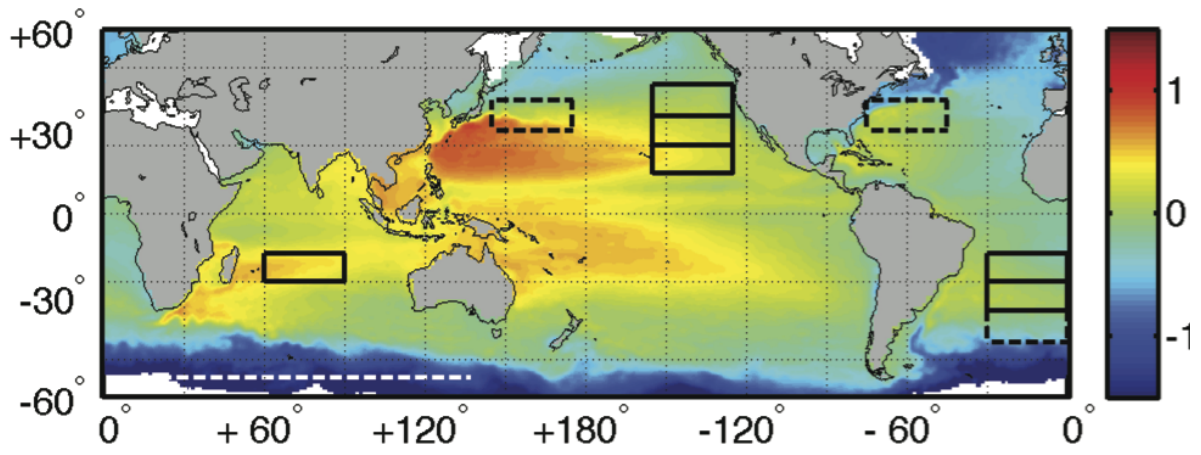

Figure 4-19: The climatological mean sea surface height in $m$ from the altimetry (color). Black boxes denote the off-equatorial regions where the $\omega-k$ spectra of the observed sea surface height from the altimetry are presented in Wortham (2012). Non-dispersive lines exist in the $\omega-k$ spectrum of sea surface height in the solid black boxes and do not exist in the dashed black boxes. It also does not exist along the white dashed line in the Southern Ocean (Ferrari and Wunsch 2010). Here $\omega$ is frequency and $k$ is zonal wavenumber.

constant. In the Gulf Stream and Kuroshio Extension regions, both westward and eastward eddy propagation dominates (Wortham, 2012); in the Southern Ocean, the eddy propagation speed is highly inhomogeneous due to the small-scale topography steering and jet meandering, etc. (Fu, 2009). These might explain the breakdown of our mechanism in these regions.

The above inference is neither definitive nor complete. We can further examine the relevance of the eddy-propagation mechanism to the ECCO2 state estimate in several ways.

a) We find, through analysis of Exp2, that non-dispersive lines exist in the $\omega-k_{0}$ spectrum, where $k_{0}$ is the wavenumer along the eddy-propagation direction. In some regions, eddy propagate velocity has a non-zonal component (Fu, 2009). Thus, one can identify the regions where $\omega-k_{0}$ spectra have non-dispersive lines in the ECCO2 model. Compared to the $\omega-k$ spectra, the $\omega-k_{0}$ spectra are more proper to test the relevance of the eddy-propagation mechanism. One 
can also characterize striations at different depth levels and vertical modes, examine whether the non-dispersive lines exist in the $\omega-k_{0}$ spectra of motions at different depth levels and vertical modes.

b) One can compare the eddy-propagation direction from the correlation method with the striation direction in the global ocean at each depth. If the eddypropagation direction is roughly homogeneous in a region and roughly aligns with the striation direction, the eddy-propagation mechanism may be relevant.

c) In Exp2, the gyre flow influences the direction of eddy propagation and striations. One might quantify the effect of the subtropical gyre flow on the striation formation by carrying our a parallel experiment to the ECCO2 state estimate. This experiment is only forced by small-scale wind forcing. Comparison of this experiment with the ECCO2 state estimate can illustrate how the gyre flow influences the striation patterns. One caveat is that the large-scale wind not only influences the eddy propagation direction, but also influences the eddy generation mechanism and the whole oceanic energy cycle. Because the gyre generated from the large-scale wind stores the mean available potential energy which is an important energy source for baroclinic instability.

\section{On the applicability of the linear theory}

Though the ocean is never exactly linear, our linear theory may still be useful in understanding the striation dynamics. Many studies show that linear theories, which are generally simpler than nonlinear ones, are very useful in understanding many aspects of the ocean, such as time-mean circulation, eddy length scales, eddy generation mechanisms and eddy phase speeds (e.g. Wunsch, 2011; Tulloch et al., 2011; Müller and Frankignoul, 1981; Frankignoul and Müller, 1979; Chelton et al., 2007). The above list of examples is far from complete. Part of the reason for the wide 
usage is that insights can be gained by comparing linear theories with observations and modeling results.

Our striation interpretation (quasi-stationary linear Rossby waves) from the linear theory (Eq. 4.14) might apply to some extent in the regions within $15^{\circ}$ of the equator. Previous studies have shown that amplitude and positions of the timemean barotropic jets are overall consistent with the linear wind-driven solutions at $5^{\circ} S-15^{\circ} S$ in the South Pacific (e.g. Taguchi et al., 2012; Kessler and Gourdeau, 2006). However, our inference here comes with caveats. First, our linear theory assumes that the energy for striations and eddies is only from the external forcing, not from eddy-eddy interaction and eddy-mean flow interaction. However, at lowlatitudes, baroclinic instabilty at the deformation radius scale can provide energy to waves (Tulloch et al., 2009). Second, the quasi-geostrophic model is not valid at the equator due to the large Rossby wave number there (e.g. Theiss and Mohebalhojeh, 2009; Mohebalhojeh and Theiss, 2011).

As to further tests of the applicability of the linear theory to the ECCO2 state estimate, one can ask to what extent striations from the ECCO2 state estimate in the global ocean resemble those in the linear solution. Study along this line can reveal quantitatively where the linear interpretation applies and the effect of nonlinearity on the striation properties. The problem can be tackled in several ways. An easy way is to solve our linearized idealized model using realistic wind forcing, parameters and domain shape, and then compare the solution with those in the thermocline of the ECCO2 state estimate. Another method is that one can solve the linear response of the ocean to the realistic wind forcing using the stratified quasi-geostrophic equation with the time-mean flow imposed. We can then compare the striations from the linear model with those from the ECCO2 state estimate. Müller and Frankignoul (1981) used a similar method to evaluate the role of wind in eddy generation. 


\subsection{Conclusions}

The main contributions of this chapter are (1) to estimate the percentage of oceanic variability associated with striations and (2) to evaluate the effect of the gyre-scale flow on striations using the frequency-wavenumber spectrum of the eddy stream function.

Using a criterion introduced in Section 4.3, we find that a non-negligible percentage of zonal velocity variability is associated with striations, which we define as banded structures in the low-frequency motions. Striations account for $49 \%(14 \%)$ of zonal velocity variability in the upper $1100 \mathrm{~m}$ in the CNP (ENP) regions from the ECCO2 state estimate. They account for more than $10 \%$ of the variability in the surface geostrophic flow in the two selected regions. The non-negligible percentage suggests that striations can probably contribute significantly to the tracer transport and energy budgets.

We examined the effect of the gyre flow in the time-mean circulation on the origin and direction of striations in both the ECCO2 model and an idealized model. In the ECCO2 state estimate, striations are embedded in the large-scale gyre flows in the ENP and CNP regions. They are non-zonal in the ENP region and zonal in the CNP region. We propose that the gyre flow contributes to the striation direction difference in the two regions. An idealized model is formulated and then solved to test this hypothesis. The directions of striations in the idealized model qualitatively resemble those in the ECCO2 state estimate. To further investigate how the gyre flow influences striations, we study the frequency-wavenumber spectrum of the eddy stream function. First, in the linearized limit, both the numerical and analytical solutions of the spectrum suggest that striations in this case are quasi-stationary linear Rossby waves and the gyre flow influences the striation direction by influencing 
the direction of the zero Rossby wave frequency curve. Second, in the nonlinear case, both the numerical analysis and our simplified spectrum model are useful in understanding striations and the gyre effect. In this case, striations arise from the temporal averaging effect of non-dispersively propagating eddies (eddy-propagation mechanism). The gyre flow influences striation properties by influencing the eddy propagation direction. We also identify the link between the non-dispersive line in the spectrum and the eddy-propagation mechanism.

Our work has some potential applications. First, we provide a new striation definition and a criterion to quantitatively extract striations from the eddy field. This criterion is useful in evaluating the role of striations to tracer transport, mixing and the energy cycle. Second, though the eddy-propagation mechanism is derived from the simple model, it is probably relevant to striations in the interior ocean between a few degrees off the equator and $45^{\circ}$, where non-dispersive lines exist in the spectra.

Many aspects about striations in the idealized model remain elusive, such as the origin/slope of the non-dispersive lines (some discussions are in Ferrari and Wunsch, 2010), the transition between the wave mechanism and eddy-propagation mechanism in the parameter space, and the quantitative estimate about the contribution of the large-scale gyre flow to the eddy propagation velocity. Some other future studies are (1) to estimate the percentage of the zonal velocity variability associated with striations and to characterize the spatial/temporal characteristics of striations in the global ocean using the ECCO2 model; (2) to estimate the contribution of striations to the eddy energy budgets, mixing and tracer transport using the ECCO2 model; (3) to test quantitatively the relevance of our striation interpretations in different oceanic regions, vertical modes and depth levels; (4) to investigate the effect of additional dynamical factors on striations using idealized models, such as the coupling of the 
vertical modes, the time-dependence of the gyre flow, the Hawaiian Island inducing beta plumes, and the eastern boundary currents inducing radiating instabilities.

\subsection{Appendix}

\subsubsection{Formal derivation of the idealized model}

Here we provide a more rigorous derivation of the idealized model, starting from the governing equations of the 1.5-layer model. To avoid confusion, note that the meaning of the symbols in this appendix only applies in this appendix. As discussed in Huang (2010), the momentum and mass conservation equations for the 1.5-layer model are

$$
\begin{gathered}
\left(\frac{\partial}{\partial t}+\mathbf{u} \cdot \nabla\right) u-f v=-g^{\prime} \frac{\partial}{\partial x} h \\
\left(\frac{\partial}{\partial t}+\mathbf{u} \cdot \nabla\right) v+f u=-g^{\prime} \frac{\partial}{\partial y} h \\
\frac{\partial h}{\partial t}+\frac{\partial}{\partial x}(h u)+\frac{\partial}{\partial y}(h v)=-w_{e},
\end{gathered}
$$

where $u$ and $v$ are zonal and meridional velocities, $g^{\prime}$ is the reduced gravity. $\mathbf{u}=u \widehat{i}+v \widehat{j}$ is the two-dimensional velocity. $\nabla=\frac{\partial}{\partial x} \widehat{i}+\frac{\partial}{\partial y} \widehat{j} . h$ represents the layer thickness and $f$ is the Coriolis parameter. Note that friction is ignored in the momentum equations for simplicity purpose. $w_{e}$ is the Ekman pumping velocity:

$$
w_{e}=\nabla \times\left(\frac{\vec{\tau}}{\rho_{0} f}\right)=\operatorname{curl}\left(\frac{\vec{\tau}}{\rho_{0} f}\right)
$$

where $\vec{\tau}$ is the wind stress and $\rho_{0}$ is the constant reference density. Cross-differentiating Eqs. 4.28 and 4.29 and using Eq. 4.30, we can get the potential vorticity equation 


$$
\left(\frac{\partial}{\partial t}+\mathbf{u} \cdot \nabla\right) \frac{f+\zeta}{h}=\frac{(f+\zeta) w_{e}}{h^{2}}
$$

where

$$
\zeta=\frac{\partial v}{\partial x}-\frac{\partial u}{\partial y}
$$

representing the relative vorticity.

As in Pedlosky (1984), we separate the variables into two parts

$$
\begin{gathered}
\mathbf{u}(\mathbf{x}, t)=U_{0}\left[\mathbf{U}(\mathbf{X}, T)+\mathbf{u}^{\prime}\left(\mathbf{x}^{\prime}, t^{\prime}, \mathbf{X}, T\right)\right] \\
h(\mathbf{x}, t)=H_{0}\left[H(\mathbf{X}, T)+\delta h^{\prime}\left(\mathbf{x}^{\prime}, t^{\prime}, \mathbf{X}, T\right)\right],
\end{gathered}
$$

$\mathbf{U}$ and $H$ are gyre scale part. $\mathbf{u}^{\prime}$ and $h^{\prime}$ are mesoscale part. $(\mathbf{X}, T)$ are the coordinate for the gyre-scale variation and $\left(\mathbf{x}^{\prime}, t^{\prime}\right)$ are the coordinate for the mesoscale-variation. The key assumption, as stated in Pedlosky (1984), is that the gyre scale is much larger and slower than the mesoscale, that is,

$$
\mathbf{X}=\mathbf{x} / L, \mathbf{x}^{\prime}=\mathbf{x} / l, t^{\prime}=\sigma t, T=t^{\prime} l / L, \delta=l / L \ll 1
$$

where $\delta$ is the scale ratio. Also, we scale the Coriolis parameter: $f^{\prime}=f(Y) / f_{0}$. Now we can write the spatial and temporal derivatives in the gyre-scale and mesoscale coordinates

$$
\frac{\partial}{\partial x}=\frac{1}{l}\left(\delta \frac{\partial}{\partial X}+\frac{\partial}{\partial x^{\prime}}\right), \frac{\partial}{\partial y}=\frac{1}{l}\left(\delta \frac{\partial}{\partial Y}+\frac{\partial}{\partial y^{\prime}}\right), \text { and } \frac{\partial}{\partial t}=\frac{U_{0}}{l}\left(\delta \frac{\partial}{\partial T}+\frac{\partial}{\partial t^{\prime}}\right) .
$$

Using Eqs. 4.33-4.36, the momentum equations (Eqs. 4.28 and 4.29) can be 
nondimensionalized as

$$
\epsilon D_{x}\left(\mathbf{U}+\mathbf{u}^{\prime}\right)+\epsilon \delta D_{X}\left(\mathbf{U}+\mathbf{u}^{\prime}\right)+f \widehat{k} \times\left(\mathbf{U}+\mathbf{u}^{\prime}\right)=-\nabla_{X}\left(H+\delta h^{\prime}\right)-\nabla_{x} h^{\prime}
$$

where $\epsilon=U_{0} / f_{0} l$ and $g^{\prime} H_{0}=f_{0} U_{0} L$. The operators $D_{X}$ and $D_{x}$ denote

$$
D_{X}=\frac{\partial}{\partial T}+\left(\mathbf{U}+\mathbf{u}^{\prime}\right) \cdot \nabla_{X}, D_{x}=\frac{\partial}{\partial t}+\left(\mathbf{U}+\mathbf{u}^{\prime}\right) \cdot \nabla_{x}
$$

Note that, the primes in $\mathbf{x}^{\prime}$ and $f^{\prime}$ are dropped in Eq. 4.37 to simplify the presentation and this simplification is also used for the rest of this appendix. The non-dimensional potential vorticity is

$$
q=\frac{f+\epsilon \zeta^{\prime}+\epsilon \delta\left(V_{X}-U_{Y}+v_{X}^{\prime}-u_{Y}^{\prime}\right)}{H+\delta h^{\prime}}=Q(\mathbf{X}, t)+\delta \tilde{q}(\mathbf{x}, t, \mathbf{X}, T)
$$

with

$$
\zeta^{\prime}=\frac{\partial v^{\prime}}{\partial x}-\frac{\partial u^{\prime}}{\partial y}, Q=\frac{f}{H}, \quad \tilde{q}=\frac{1}{H+\delta h^{\prime}}\left[\frac{\epsilon}{\delta} \zeta^{\prime}-\frac{f}{H} h^{\prime}+\epsilon\left(V_{X}-U_{Y}+v_{X}^{\prime}-u_{Y}^{\prime}\right)\right]
$$

We can then get the non-dimensional potential vorticity equation from Eqs. 4.32 and 4.38:

$$
D_{X} Q+D_{x} \tilde{q}+\delta D_{X} \tilde{q}=F_{X}+F_{x}^{\prime}
$$

where $F_{X}$ is the gyre-scale forcing and $F_{x}^{\prime}$ is the meso-scale forcing.

$$
F_{X}+F_{x}^{\prime} \approx \frac{w_{e 0} L}{H_{0} U} \frac{f}{H^{2}}\left(W_{e}+w_{e}^{\prime}\right)
$$

where $w_{e 0}\left[W_{e}(\mathbf{X}, T)+w_{e}^{\prime}\left(\mathbf{x}^{\prime}, t^{\prime}, \mathbf{X}, T\right)\right]=w_{e}$.

Assuming $\delta \sim \epsilon \ll 1$, the lowest order momentum and potential vorticity equa- 
tions are

$$
\begin{gathered}
f \widehat{k} \times \mathbf{U}+f \widehat{k} \times \mathbf{u}^{\prime}=-\nabla_{X} H-\nabla_{x} h^{\prime}, \\
{\left[\frac{\partial}{\partial T}+\mathbf{U} \cdot \nabla_{X}\right] Q+\mathbf{u}^{\prime} \cdot \nabla_{X} Q+\left[\frac{\partial}{\partial t}+\left(\mathbf{U}+\mathbf{u}^{\prime}\right) \cdot \nabla_{x}\right] q^{\prime}=F_{X}+F_{x}^{\prime},}
\end{gathered}
$$

where

$$
q^{\prime}=\frac{1}{H}\left(\frac{\epsilon}{\delta} \zeta^{\prime}-\frac{f}{H} h^{\prime}\right)
$$

Eqs. 4.42 and 4.43 can be separated into one part independent of $\mathrm{x}$ and the other part dependent of $\mathbf{x}$. Setting each part to zero, we can get the leading order balances for gyre-scale motions $\mathbf{U}$ and the leading order balances for meso-scale motions $\mathbf{u}^{\prime}$, as listed below:

$$
\begin{gathered}
f \widehat{k} \times \mathbf{U}=-\nabla_{X} H, \\
{\left[\frac{\partial}{\partial T}+\mathbf{U} \cdot \nabla_{X}\right] Q=F_{X},} \\
f \widehat{k} \times \mathbf{u}^{\prime}=-\nabla_{x} h^{\prime}, \\
{\left[\frac{\partial}{\partial t}+\left(\mathbf{U}+\mathbf{u}^{\prime}\right) \cdot \nabla_{x}\right] q^{\prime}+\mathbf{u}^{\prime} \cdot \nabla_{X} Q=F_{x}^{\prime},}
\end{gathered}
$$

\subsubsection{Calculation of eddy propagation velocity}

The correlation method used to diagnose the eddy propagation velocity in this chapter is similar to the maximum cross-correlation method in $\mathrm{Fu}$ (2009). To avoid confusion, note that the meaning of the symbols in this appendix only applies in this appendix.

Let $A(x, y, t)$ be a variable representing the flow field, such as eddy kinetic energy or eddy stream function. In a spatial domain with width $W$ and length $L$, the cross correlation can be computed:

$$
C(x, y, t, \delta x, \delta y, \delta t)=\overline{A(x, y, t) A(x+\delta x, y+\delta y, t+\delta t)},
$$


where ${ }^{-}$is the spatial average over the spatial window. Define $C_{0}(x, y, t, \delta t)$ as the maximum cross correlation for all possible choices of $(\delta x, \delta y)$ at the location and time $(x, y, t)$ and time lag $\delta t$; also define $\left(\delta x_{0}(x, y, t, \delta t), \delta y_{0}(x, y, t, \delta t)\right)$ as the spatial lags at $C_{0}(x, y, t, \delta t)$.

When computing the eddy propagating velocity in the ECCO2 state estimate, A is the eddy kinetic energy, where eddies are defined as the deviation from the 16-year average (1992-2007). The spatial window is a small patch centered at $(x, y)$ with the size of $4^{o} \times 4^{o}$, but $\delta t$ has multiple values, denoted by $\delta t_{n}$. The eddy propagating velocity is determined by

$$
\overrightarrow{V e l}_{e d d y}(x, y, t)=\frac{\sum_{n}\left(\delta x_{0}\left(x, y, t, \delta t_{n}\right) / \delta t_{n} \vec{i}+\delta y_{0}\left(x, y, t, \delta t_{n}\right) / \delta t_{n} \vec{j}\right) C_{0}\left(x, y, t, \delta t_{n}\right)}{\sum_{n} C_{0}\left(x, y, t, \delta t_{n}\right)},
$$

and the temporally averaged eddy propagating velocity is $\overrightarrow{\overrightarrow{V e}}_{e d d y}(x, y, t)$.

When computing the eddy-propagating velocity in the quasi-geostrophic experiment with a double gyre, $\mathrm{A}$ is the stream function $\psi$, the spatial window is a small patch centered at $(x, y)$ and $\delta t$ is a constant value $\delta t_{0}$. The eddy propagating velocity is determined by

$$
\overrightarrow{V e l}_{e d d y}(x, y, t)=\left(\delta x_{0}\left(x, y, t, \delta t_{0}\right) / \delta t_{0} \vec{i}+\delta y_{0}\left(x, y, t, \delta t_{n} 0 / \delta t_{0} \vec{j}\right) .\right.
$$




\title{
Chapter 5
}

\section{Striations and their contribution to}

\author{
the energy budget and tracer
}

\section{mixing in the barotropic system}

\section{with mean flow}

\subsection{Introduction}

Chapter 4 shows that striations are pervasive in the ocean basins and they account for a non-negligible percentage of zonal velocity variability. Thus, we suspect that striations can have significant consequences to the ocean general circulation in the aspect of tracer mixing and energy budgets. This chapter focuses on studying striations' consequences in these two aspects using a barotropic quasi-geostrophic model on a beta plane. Our specific goals are (1) to develop diagnostic framework and use them to evaluate striations' contribution to mixing and energy budget, and (2) to characterize and interpret the effect of mean flow on striations in the barotropic 
system.

There are several reasons why we choose the barotropic quasi-geostrophic model on a beta plane. First, it is one of the simplest systems producing banded structures and quite a few aspects about the eddy field in this system are known. For example, Rhines jets can arise due to the arrest of inverse cascade by beta effect (e.g. Rhines, 1975). A kinematic interpretation of Rhines jets is the mixing of potential vorticity by eddies (e.g. Dritschel and McIntyre, 2008). These existing theories are useful for us in understanding striations and their consequences. Second, the simple model is a good tool to test the validity of our diagnostic framework due to its computational efficiency and the ease of interpretation. Third, recent studies demonstrate that this model, though idealized, is still a useful tool for theoretical explorations about banded structures and many related frontiers are still open (e.g. Srinivasan and Young, 2012; Farrell and Ioannou, 2007; Danilov and Gurarie, 2004; Danilov and Gryanik, 2004).

Though the model is a highly simplified representation of the ocean, it produces interesting results and our diagnostic framework is applicable in more complicated systems. We find that whether Rhines jets are striations or not depends on the imposed mean flow direction; the contribution of striations to mixing and the energy budget is also sensitive to the imposed mean flow direction. Our results may not be directly applicable to barotropic striations in the ocean, since the barotropic mode in the ocean is coupled with and extracts energy from the baroclinic mode, whereas our model only has a single mode forced by external forcing. However, our diagnostic framework is applicable to investigating the contribution of realistic striations to mixing and the global energy budgets (details about the applications of this chapter are in Section 5.6).

This chapter is organized as follows. Section 5.2 is about the model setup. Section 5.3 compares the Rhines jets with striations, and compares the striation interpreta- 
tions in this chapter with the eddy-propagation mechanism from Chapter 4. Sections 5.4 and 5.5 present our key results: the formulation of the diagnostic framework and the contribution of striations to mixing and energy budgets. We also present theories about the effect of mean flow on total mixing and energy cascades in Sections 5.4 and 5.5. Section 5.6 discusses the application. Conclusions are given in Section 5.7.

\subsection{Experiment setup}

We start from the idealized model used in Chapter 4:

$$
\left(\frac{\partial}{\partial t}+\bar{U} \frac{\partial}{\partial x}+\bar{V} \frac{\partial}{\partial y}\right) \tilde{q}+J(\psi, \tilde{q})+J(\psi, Q)=\mathcal{F}(x, y, t)-r \cdot \tilde{q}
$$

As stated in Chapter $4, \psi$ denotes the eddy stream function, $\tilde{q}=\nabla^{2} \psi-F_{1} \psi$ the eddy potential vorticity $(\mathrm{PV})$, and $J$ is the Jacobian operator. $F_{1}$ is the squared inverse of the deformation radius $R d, Q=\beta y+F_{1}(\bar{U} y-\bar{V} x)$ represents the large-scale potential vorticity, and $r$ is the friction coefficient. Different from Chapter $4, \bar{U}$ and $\bar{V}$ here is the imposed constant mean flow, not the gyre flow; the equation is solved using the "pseudo-spectral" method in a doubly-periodic domain, not in a channel. The numerical method is described in detail in Arbic (2000). The doubly-periodic domain is discretized at $128 \times 128$ grid points and the domain size is $3000 \mathrm{~km} \times 3000 \mathrm{~km}$.

$\mathcal{F}(x, y, t)$ represents the random forcing and has the same formula as that in Chapter 4 (Eq. 4.10 and Eq. 4.11). As in Chapter 4, the forcing has a narrow banded spectrum; the memory coefficient is 0.7 ; thus the forcing decorrelates rapidly and is approximately white noise. ${ }^{1}$ The forcing has a dominant spatial scale of 60

\footnotetext{
${ }^{1}$ In the barotropic quasi-geostrophic model with external forcing, the Galilean invariance holds if the system is forced by white noise. Galilean invariance is useful in the interpretation of mean flow's effect on Rhines jets, energy cascades and spatially averaged mixing. These are discussed later in the chapter.
} 
Table 5.1: The list of experiments.

\begin{tabular}{lrrr}
\hline \hline & Exp1 & Exp2 & Exp3 \\
\hline $\bar{U}(m / s)$ & 0 & 0 & -0.12 \\
$\bar{V}(m / s)$ & 0 & 0.12 & 0 \\
$R d\left(10^{3} m\right)$ & $\infty$ & $\infty$ & $\infty$ \\
$r\left(10^{-9} s^{-1}\right)$ & 7 & 7 & 7 \\
$\beta\left(10^{-11} m^{-1} s^{-1}\right)$ & 2 & 2 & 2 \\
\hline
\end{tabular}

$\mathrm{km}$. The amplitude of the forcing $(\tilde{A})$ is $2.7 \times 10^{-11} \mathrm{~s}^{-2}$, which is strong enough to make the system nonlinear. The time step is $1 / 32$ day.

Chapter 4 examined the striation property in the case with finite deformation radius. This chapter focuses on the case with the infinite deformation radius. Table 5.1 summarizes the three experiments analyzed in this chapter. Exp1 has no mean flow imposed and represents the original case in Rhines (1975), Exp2 has northward mean flow imposed, and Exp3 has westward mean flow imposed. All the experiments start from rest and they are run for another 100 years after the statistical equilibrium states have been reached. The analysis in this chapter uses the 100-year output in the statistical equilibrium state.

\subsection{Rhines jets vs. striations}

\subsubsection{Rhines jets}

The existence and direction of Rhines jets do not depend on the imposed mean flow in our experiments (Figure 5-1). In the barotropic quasi-geostrophic model on a beta plane with no mean flow imposed, Rhines jets exist in the eddy snapshot as the elongated structures (Rhines, 1975). Consistently, Rhines jets show up in the snapshots of our experiments and they are all roughly elongated in the zonal direction 
(Figure 5-1). However, Rhines jets in Exp2 are advected northward by the imposed northward mean flow (Figure 5-2).
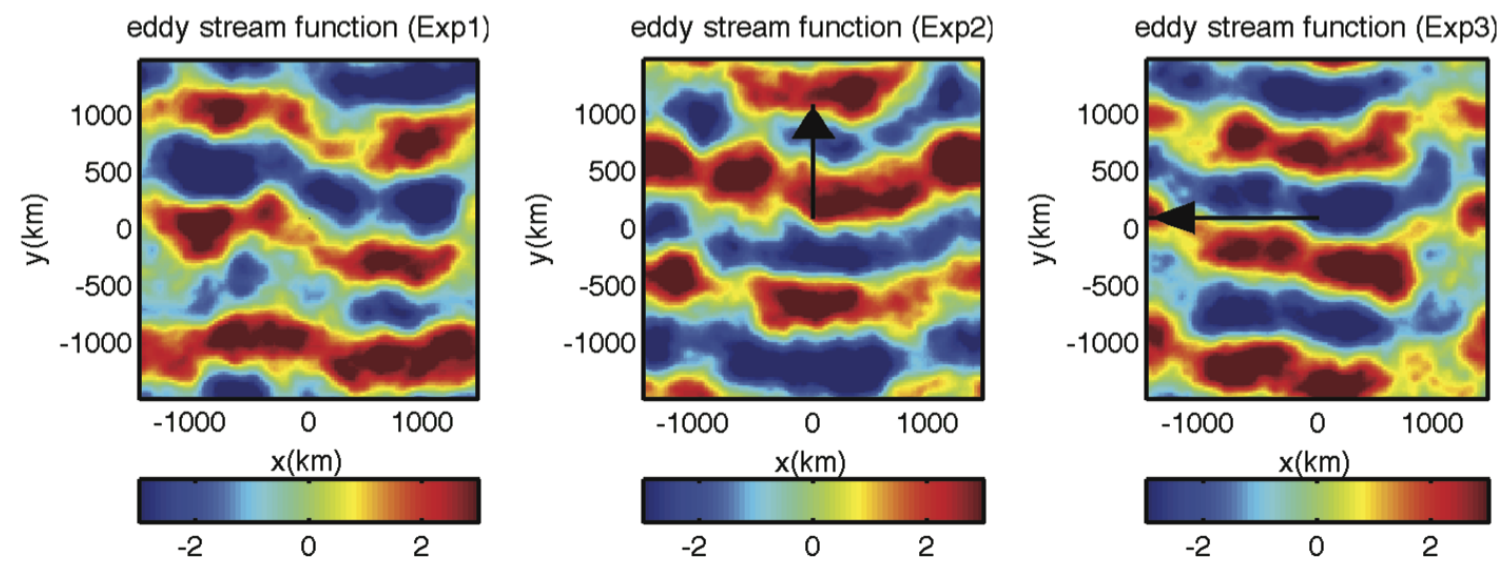

Figure 5-1: Snapshots of the total eddy stream function $\psi\left(10^{4} \mathrm{~m}^{2} / \mathrm{s}\right)$ in the doublyperiodic domain from Exp1 (left), Exp2 (middle) and Exp3 (right). Black arrows denote the imposed mean flow direction in each experiment. $x$ represents zonal distance and $y$ represents meridional distance. Rhines jets are robust features in the eddy snapshots of all the three experiments.
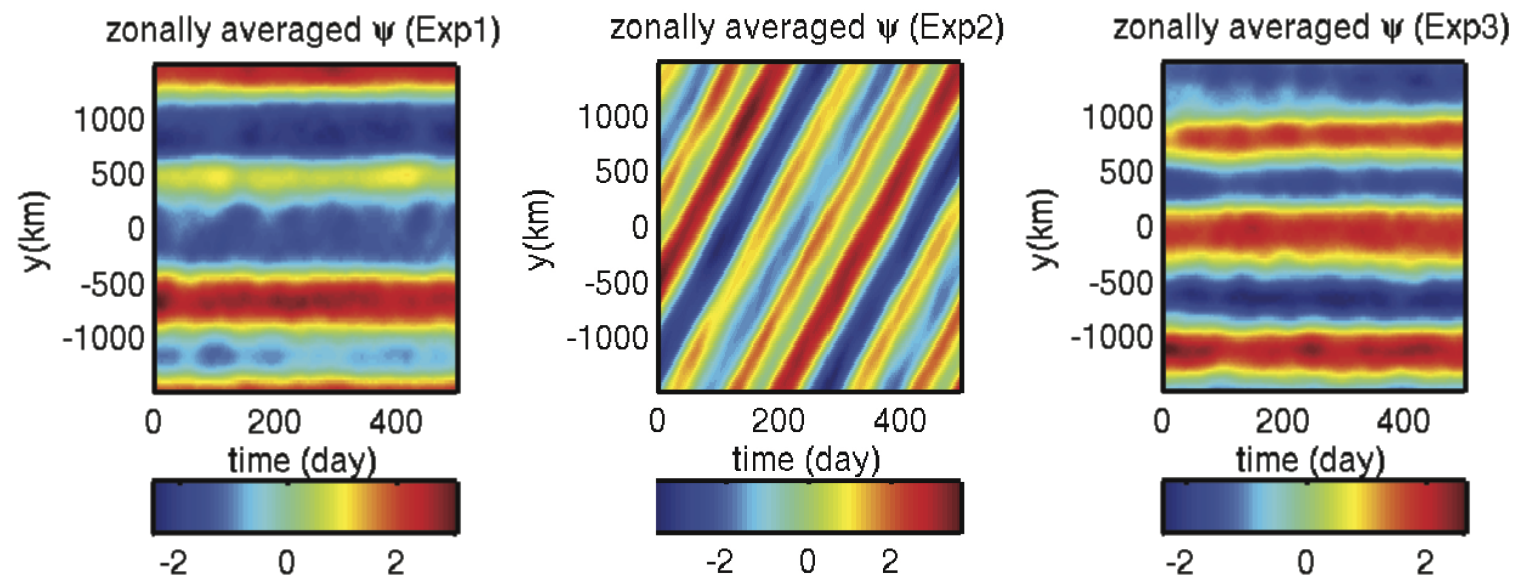

Figure 5-2: Hovmöller (time-meridional distance) plots of the zonally averaged eddy stream function $\psi\left(10^{4} \mathrm{~m}^{2} / \mathrm{s}\right)$ in each experiment. This figure uses the zonally averaged eddy stream function as an approximate indicator of Rhines jets. Rhines jets are advected northward by the imposed northward mean flow in Exp2. The meridional drifts of the jets are invisible over the 500 day time scale in Exp1 and Exp3 due to the absence of meridional component of the imposed mean flow.

The Galilean invariance of the barotropic model can explain why the generation 
and direction of Rhines jets do not depend on the imposed mean flow. The PV equation for the barotropic quasi-geostrophic decaying turbulence on a beta plane is

$$
\frac{\partial}{\partial t} \nabla^{2} \psi+J\left(\psi-\bar{U} y+\bar{V} x, \nabla^{2} \psi\right)+\beta \frac{\partial}{\partial x} \psi=0
$$

Define a moving coordinate $\left(x^{\prime}, y^{\prime}, t^{\prime}\right)$ :

$$
x^{\prime}=x-\bar{U} t, y^{\prime}=y-\bar{V} t \text { and } t^{\prime}=t .
$$

Then, we get Eq. 5.2 in the coorindates $\left(x^{\prime}, y^{\prime}, t\right)$ :

$$
\frac{\partial}{\partial t^{\prime}} \nabla^{2} \psi+\frac{\partial \psi}{\partial x^{\prime}} \frac{\partial q}{\partial y^{\prime}}-\frac{\partial \psi}{\partial y^{\prime}} \frac{\partial q}{\partial x^{\prime}}+\beta \frac{\partial \psi}{\partial x^{\prime}}=0
$$

which is the same as the original Rhines jet model, where no mean flow is imposed. External forcing in Exp1, Exp2 and Exp 3 is of small spatial scale and of short decorrelation time scale; thus the basic statistical behavior of large eddies in the three experiments is the same as those in the decaying turbulence. Thus, eddies in Exp2 and Exp3 in the coordinates $\left(x^{\prime}, y^{\prime}, t^{\prime}\right)$ have the same characteristics as eddies in Exp1 in the coordinates $(x, y, t)$ : the dominant eddies become "zonal" when energy cascades to low "wavenumbers" and low "frequencies" (here the "wavenumber" and "frequency" is defined in the coordinates $\left.\left(x^{\prime}, y^{\prime}, t^{\prime}\right)\right)$; Rhines jets are generated and they align with the $x^{\prime}$ direction. Note that $x^{\prime}\left(y^{\prime}\right)$ and $x(y)$ are in the same direction. Therefore, the generation and direction of Rhines jets do not depend on the imposed constant mean flow. 


\subsubsection{Frequency-wavenumber spectrum}

Striations are not necessarily Rhines jets (Section 5.3.3). Following Chapter 4, we use the spectral approach to interpret striations. Thus, here we describe some properties of the spectrum as a preparation for the striation discussion.

We can obtain the spectrum of the eddy stream function in the case with mean flow imposed from that in the case with no mean flow. We define $\phi_{A}(x, y, t)$ as the solution to the experiment with no mean flow (Exp1) and define its spectrum as $\left|\widehat{\phi}_{A}(k, l, \omega)\right|^{2}$ with

$$
\left.\widehat{\phi}_{A}(k, l, \omega)=\iiint \phi_{A}(x, y, t) e^{-i(k x+l y-\omega t}\right) d x d y d t .
$$

According to Eqs. 5.2 and 5.3, the function

$$
\phi_{B}(x, y, t)=\phi_{A}(x-\bar{U} t, y-\bar{V} t, t)
$$

have the same statistical behavior as the solution to the experiment with the mean flow $(\bar{U}, \bar{V})$.

Using Eq. 5.5, we obtain the spectrum of $\phi_{B}(x, y, t)$ :

$$
\left|\widehat{\phi}_{B}(k, l, \omega)\right|^{2}=\left|\widehat{\phi}_{A}(k, l, \omega-k \bar{U}-l \bar{V})\right|^{2},
$$

with

$$
\left.\widehat{\phi}_{B}(k, l, \omega)=\iiint \phi_{B}(x, y, t) e^{-i(k x+l y-\omega t}\right) d x d y d t .
$$

Thus, shifting the frequency spectrum of $\phi_{A}(x, y, t)$ at $(k, l)$ by $k \bar{U}+l \bar{V}$, we obtain the frequency spectrum of $\phi_{B}(x, y, t)$ at $(k, l)$. The comparison of the frequency spectrum at each $(k, l)$ in Exp1 with the frequency spectrum at the same $(k, l)$ in $\operatorname{Exp} 2$ and 
Exp3 demonstrates that this is indeed valid.

The frequency-wavenumber spectrum suggests that eddies in our experiments can be viewed as a set of weakly interacting Rossby waves. ${ }^{2}$ Figure 5-3 shows the frequency-wavenumber spectrum in $\operatorname{Exp} 1\left(\left|\widehat{\phi}_{A}(k, l, \omega)\right|^{2}\right){ }^{3}$ In the barotropic turbulence on a beta plane, energy cascades to large scales and motions with spatial scales larger than a critical scale (roughly the Rhines scale) behave like waves (Rhines, 1975). Consistently, we find that the large values of the spectra (red surface) roughly lie on the surface of the Rossby wave dispersion relation (black mesh surface). $\left|\widehat{\phi}_{A}(k, l, \omega)\right|^{2}$ integrated over the red surface accounts for $90 \%$ of the integral of $\left|\widehat{\phi}_{A}(k, l, \omega)\right|^{2}$ over the entire $k-l-\omega$ space. Thus, a large portion of the motions in Exp1 satisfies the Rossby wave dispersion relation and the eddy field can be viewed as a group of Rossby waves. This statement is also valid for Exp2 and Exp3, as both the frequency spectrum at each $(k, l)$ and the Rossby wave frequency is shifted by $k \bar{U}+l \bar{V}$, compared to the case with no mean flow imposed.

We find that the wavenumber spectrum of the eddy stream function does not depend on the imposed mean flow, since

$$
\int_{-\infty}^{\infty}\left|\widehat{\phi}_{B}(k, l, \omega)\right|^{2} d \omega=\int_{-\infty}^{\infty}\left|\widehat{\phi}_{A}(k, l, \omega-k \bar{U}-l \bar{V})\right|^{2} d \omega=\int_{-\infty}^{\infty}\left|\widehat{\phi}_{A}(k, l, \omega)\right|^{2} d \omega .
$$

Again, the wavenumber spectra in our experiments are consistent with this statement, given the expected variation from realization to realization.

\footnotetext{
${ }^{2}$ Rossy waves here refer to eddy motions satisfying the dispersion relation of Rossby waves.

${ }^{3}$ Wortham (2012) discusses how to compute the frequency-wavenumber spectra.
} 

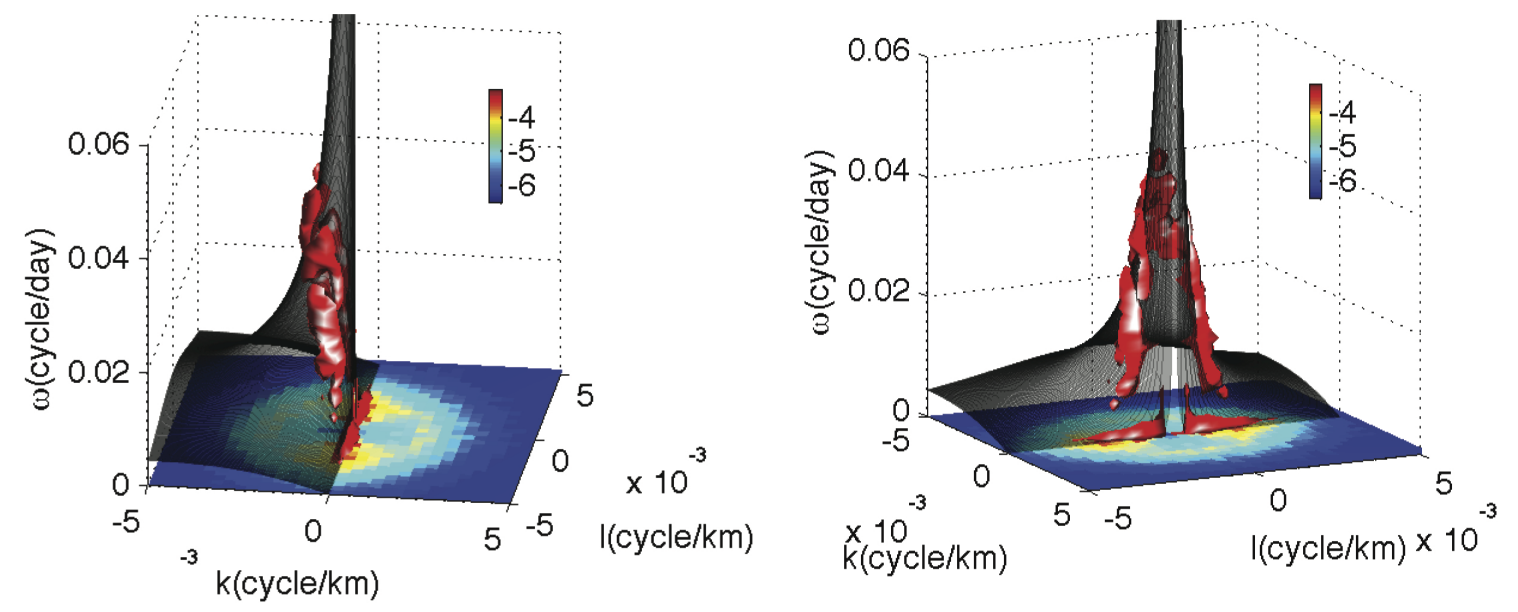

Figure 5-3: The normalized three-dimensional frequency-wavenumber spectrum of the eddy stream function in Exp1 viewed from two different angles. Slices show the three-dimensional spectra through the plane $\omega=0$. Black mesh surfaces are those of the dispersion relation of the barotropic Rossby waves. Points with the normalized power larger than $10^{-3.2}$ are indicated in red. Colorbars are on the logarithmic scale.

\subsubsection{Interpretation about barotropic striations}

Striations exist in all three experiments and they are not necessarily dominated by Rhines jets (Figure 5-4). In Exp1, their direction, width and magnitude are roughly the same as Rhines jets (Figure 5-1). This indicates that when there is no mean flow imposed, striations are dominated by Rhines jets. In Exp2, the imposed mean flow is in the meridional direction; striations are tilted northwestward and their amplitude is much weaker than those of Rhines jets (Figure 5-1). As in Exp1, the direction, width and magnitude of striations in Exp3 are roughly the same as Rhines jets, indicating that striations in Exp3 are also dominated by Rhines jets. Next, we explain these phenomena.

Striations are dominated by a group of quasi-stationary Rossby waves: this is because eddy motions can be viewed as a set of Rossby waves (Figure 5-3) and striations pick out the energy which is near the zero (shifted) frequency. Figure 5-5 

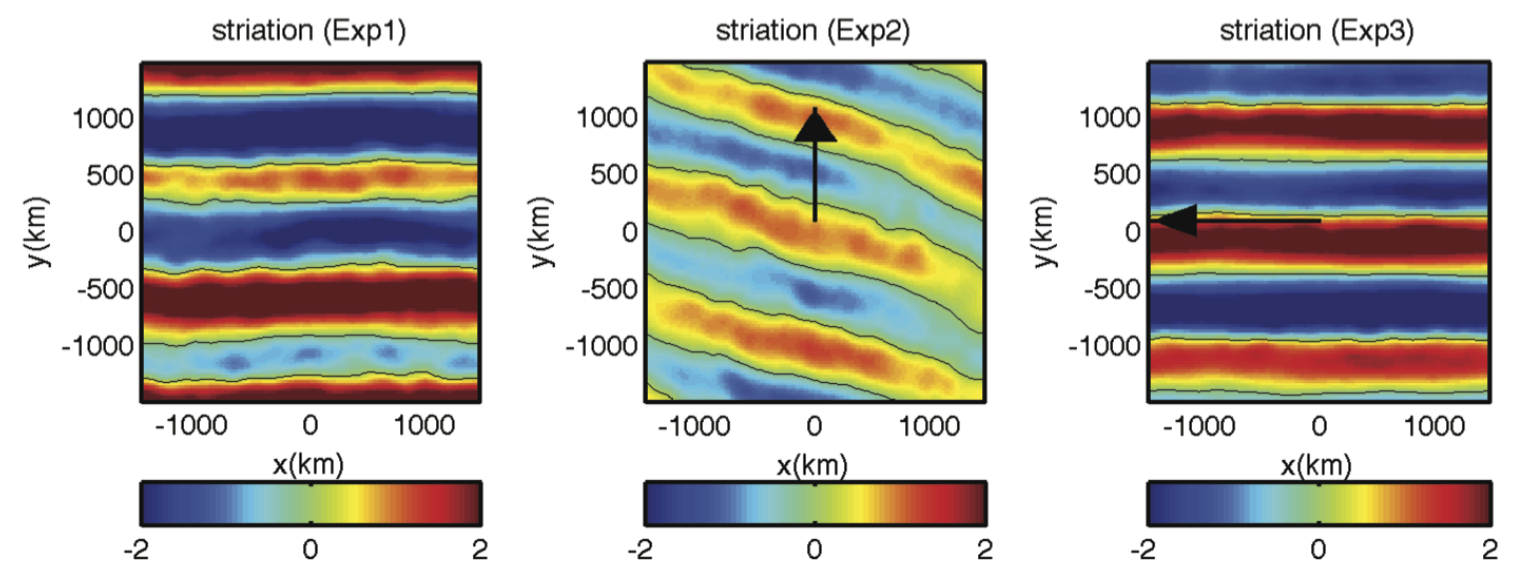

Figure 5-4: Snapshots of the part of the eddy stream function with frequency lower than $\Omega_{S}\left(10^{4} \mathrm{~m}^{2} / \mathrm{s}\right)$ in the doubly-periodic domain in each experiment. We choose $\Omega_{S}=1 / 600$ cycle/day in order to put Rhines jet in Exp2 into the high-frequency part and to illustrate a case that Rhines jets do not show up in the temporal average of oceanic motions over some long time length. Black arrows denote the imposed mean flow direction in each experiment.

further illustrates this statement. Wavenumbers with large values on the spectra, especially the dominant wavenumber of striations, indeed lie on the zero Rossby wave frequency curve. Note that, in the case with $\bar{V} \neq 0(\operatorname{Exp} 2)$, the zero Rossby wave frequency curve deviates from $k=0$; thus $k_{s} \neq 0$ and striations are non-zonal. While in the case with $\bar{V}=0$ ( Exp1 and Exp3), the zero Rossby wave frequency curve is $k=0$; thus $k_{s}=0$ and striations are zonal.

Whether Rhines jets belong to striations depends on the frequency of Rhines jets. Rhines jets are elongated in the zonal direction and they are located along $k=0$ in the wavenumber space. When $\bar{V}=0$ ( Exp1 and Exp3), the Rossby wave frequency for Rhines jets is close to zero; thus Rhines jets are low-frequency motions and striations are dominated by them. However, when $\bar{V} \neq 0$ (Exp2), Rhines jets move meridionally and their frequency is shifted from zero. In this case, Rhines jets are not part of the striations. Since most energy in this turbulence system occurs at $k=0$ due to the beta effect, striations have less energy if Rhines jets are not part of the striations. 

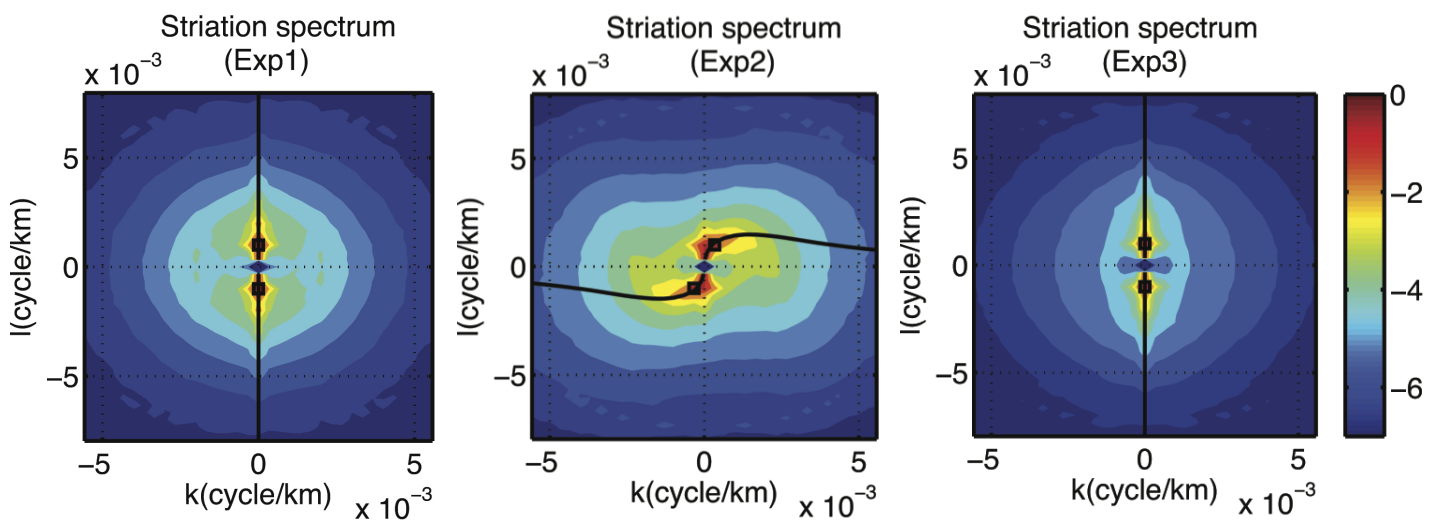

Figure 5-5: Normalized wavenumber spectrum of the part of the eddy stream function with frequency lower than $\Omega_{S}$ on the logarithmic scale in Exp1 (left), Exp2 (middle) and Exp3 (right). Black boxes denote the location of the dominant wavenumber $\left(k_{s}, l_{s}\right)$, where the wavenumber spectrum reaches its maximum value. Large values of the spectra lie on the zero Rossby wave frequency curve, denoted by the black line.

\subsubsection{Does eddy-propagation mechanism from Chapter 4 ap- ply here?}

The eddy-propagation mechanism identified from the experiments with finite deformation radius in Chapter 4 is not the right way to interpret striations in our barotropic experiments. This is because, in our barotropic experiments, energy is mostly concentrated on the surface of barotropic Rossby wave dispersion relation and thus eddies propagate dispersively. It is more reasonable to interpret striations in our barotropic experiments as quasi-stationary Rossby waves.

We find that the striation direction and the eddy-propagation direction in Exp2 are different. This difference further suggests that the eddy-propagation mechanism does not apply here. If the eddy-propagation mechanism applies, the non-dispersive line exists and striations align with the eddy propagation direction (Chapter 4). Since striations are perpendicular to striations' dominant wavenumber, we obtained the time series of the striation direction from the dominant wavenumber of striations at every snapshot. Using the correlation method described in Chapter 4, we obtained the 
time series of eddy-propagation direction. We find that, in Exp2, the angle between the time-mean striation direction and the west is $10^{\circ} \pm 3^{\circ}$, but the angle between the time-mean eddy-propagation direction and the west is $23^{\circ} \pm 1^{\circ} .{ }^{4}$ Thus, the difference between the eddy-propagation direction and the striation direction is significant.

\subsection{Effect of mean flow on the energy pathway}

This section discusses the effect of mean flow on energy cascade characteristics and striations' energy budget. Here we developed a diagnostic framework to investigate the temporal energy cascades and striations' energy budget, and formulated a theory about the effect of mean flow on temporal and spatial energy cascade characteristics. We find that, in the barotropic quasi-geostrophic turbulence on a beta plane, spatial energy cascades do not depend on the imposed mean flow, but the energy budget of striations and temporal energy cascades are sensitive to the imposed mean flow.

\subsubsection{Diagnostic framework}

The equations for temporal and spatial energy cascades are

$$
\begin{gathered}
\frac{\partial}{\partial t}<\overline{K E_{\Omega}^{<}}>=<\overline{\Pi_{\Omega}}>+<\overline{\mathcal{F}_{\Omega}}>+<\overline{D_{\Omega}}> \\
\frac{\partial}{\partial t}<\overline{K E_{K}^{<}}>=<\overline{\Pi_{K}}>+<\overline{\mathcal{F}_{K}}>+<\overline{D_{K}}>,
\end{gathered}
$$

where $\langle\cdot\rangle$ is the spatial average over the doubly-periodic domain and $\cdot$ indicates the temporal average. The meaning of each term in Eqs. 5.9 and 5.10 is listed in Table 5.2. The terms on the left-hand side represent the temporal change rates of the energy reservoirs. The energy flux to the lower wavenumber or lower frequency

\footnotetext{
${ }^{4}$ The error here is one standard error, calculated using the method from Section 3.6.3.
} 
motions through eddy-eddy interaction is balanced by external forcing and friction. Note that these equations are only valid in the barotropic quasi-gesotrophic system in the doubly-periodic domain. The mean flow $(\bar{U}, \bar{V})$ does not explicitly enter in the diagnosis equations due to the doubly-periodic boundary condition. Details about our diagnostic framework are provided in the appendix (Section 5.8.1).

The energy budget equation for striations can be obtained from Eq. 5.9. In the frequency-wavenumber space, striations are the part of the low-frequency motions inside of the narrow optimum ellipse (Chapter 4). In the three experiments, more than $84 \%$ of the low-frequency motions are in the narrow optimum ellipse. Thus, it is reasonable to use all the low-frequency motions as an approximation of striations. The energy budget of striations is therefore approximately the energy budget of lowfrequency motions:

$\frac{\partial}{\partial t}<\overline{K E_{\Omega_{S}}^{<}}>=<\overline{\Pi_{\Omega_{S}}}>+<\overline{\mathcal{F}_{\Omega_{S}}}>+<\overline{D_{\Omega_{S}}}>$

where $\Omega_{S}$ is the separation frequency between low-frequency and high-frequency motions.

Our diagnostic framework is different from those in previous studies in some aspects. Our derivation of the diagnostic equation for spatial energy cascades (Eq. 5.10) follows Scott and Wang (2005). Arbic et al. (2012) discussed the temporal energy cascades; however, their diagnostic framework does not include the term about the local change rate $\left(\frac{\partial}{\partial t}<K E_{\Omega}^{<}>\right)$, which is important if one studies the temporal variability of striations. Also, our derivation about temporal energy cascades is more intuitive compared to theirs. Assuming the spectrum gap exists between low-frequency and high-frequency motions, Thompson and Richards (2011) derived an approximated energy budget equation for low-frequency motions in order to study the low-frequency 
Table 5.2: Terms in the energy diagnostic equations (Eqs. 5.9 and 5.10). $a_{\Omega}^{<}$denotes the part of $a$ with frequencies lower than $\Omega$ and $a_{K}^{<}$denotes the part of $a$ with wavenumbers lower than $K$.

\begin{tabular}{lll}
\hline \hline term & mathematical form & meaning \\
\hline$K E_{\Omega}^{<}$ & $\frac{1}{2}\left(\nabla \psi_{\Omega}^{<} \cdot \nabla \psi_{\Omega}^{<}\right)$ & $\begin{array}{l}\text { Kinetic energy density from motions with frequencies } \\
\text { lower than } \Omega\end{array}$ \\
$\Pi_{\Omega}$ & $\psi_{\Omega}^{<} \cdot\left[J\left(\psi, \nabla^{2} \psi\right)\right]_{\Omega}^{<}$ & $\begin{array}{l}\text { Energy flux to the } K E_{\Omega}^{<} \text {reservoir due to eddy-eddy in- } \\
\text { teraction }\end{array}$ \\
$\mathcal{F}_{\Omega}$ & $-\psi_{\Omega}^{<} \cdot[\mathcal{F}(x, y, t)]_{\Omega}^{<}$ & $\begin{array}{l}\text { Energy input into the } K E_{\Omega}^{<} \text {reservoir from the external } \\
\text { forcing }\end{array}$ \\
$D_{\Omega}$ & $-r \cdot K E_{\Omega}^{<}$ & Sink of $K E_{\Omega}^{<}$due to friction \\
\hline$K E_{K}^{<}$ & $\frac{1}{2}\left(\nabla \psi_{K}^{<} \cdot \nabla \psi_{K}^{<}\right)$ & $\begin{array}{l}\text { Kinetic energy density from motions with wavenumbers } \\
\text { lower than } K\end{array}$ \\
$\Pi_{K}$ & $\psi_{K}^{<} \cdot\left[J\left(\psi, \nabla^{2} \psi\right)\right]_{K}^{<}$ & $\begin{array}{l}\text { Energy flux to the } K E_{K}^{<} \text {reservoir due to eddy-eddy in- } \\
\text { teraction }\end{array}$ \\
$\mathcal{F}_{K}$ & $-\psi_{K}^{<} \cdot[\mathcal{F}(x, y, t)]_{K}^{<}$ & $\begin{array}{l}\text { Energy input into the } K E_{K}^{<} \text {reservoir from the external } \\
\text { forcing }\end{array}$ \\
$D_{K}$ & $-r \cdot K E_{K}^{<}$ & Sink of $K E_{K}^{<}$due to friction \\
\hline
\end{tabular}

jets in the Southern Ocean. However, this spectrum gap assumption is not used in our diagnostic equation about striations' energy budget (Eq. 5.11).

\subsubsection{Results}

\section{Effect of mean flow on energy cascades}

Using both theories and numerical analysis, we find that the energy cascade in the wavenumber domain does not depend on the mean flow, but the energy cascade in the frequency domain is sensitive to the mean flow. This simple and yet interesting property has not been identified in previous studies, to our best knowledge. Using the Galilean invariance of the barotropic model, $<\overline{\Pi_{K}(x, y, t)}>$ and $<\overline{\Pi_{\Omega}(x, y, t)}>$ in the case with any arbitrary mean flow imposed can be calculated from the eddy field in the case with no mean flow imposed:

$$
<\overline{\Pi_{K}(x, y, t)}>=\int \widehat{\left[\phi_{A}\right.}(k, l, \omega) \widehat{J_{A}}(-k,-l,-\omega) H_{1}^{2}(k, l, K) d k d l d \omega
$$




$$
<\overline{\Pi_{\Omega}(x, y, t)}>=\int \widehat{\phi_{A}}(k, l, \omega) \cdot \widehat{J_{A}}(-k,-l,-\omega) \cdot H_{2}^{2}(\omega+k \bar{U}+l \bar{V}, \Omega) d k d l d \omega
$$

where $\widehat{\cdot}$ is the Fourier transform and $\phi_{A}(x, y, t)$ is the eddy stream function in the case with no mean flow imposed. $J_{A}=J\left(\phi_{A}, \nabla^{2} \phi_{A}\right)$, where $J$ is the Jacobian operator. $H_{1}(k, l, K)$ and $H_{2}(\omega, \Omega)$ are respectively the spatial-low-pass filter and temporal-lowpass filter, such as the box-car filter:

$$
H_{1}(k, l, K)=\frac{\sin (\pi k / K)}{\pi k / K} \frac{\sin (\pi l / K)}{\pi l / K}, H_{2}(\omega, \Omega)=\frac{\sin [\pi \omega / \Omega]}{\pi \omega / \Omega},
$$

where $K$ is the wavenumber $\sqrt{k^{2}+l^{2}}$ and $\Omega$ is the frequency. Details are provided in the appendix (Section 5.8.2). Thus, $<\overline{\Pi_{\Omega}(x, y, t)}>$ depends on the imposed mean flow, whereas $<\overline{\Pi_{K}(x, y, t)}>$ does not. Analysis of the three experiments demonstrates that the above statement is valid (Figures 5-6 and 5-7): $\left\langle\overline{\Pi_{K}}>\right.$ is the same in the three experiments, but $<\overline{\Pi_{\Omega}}>$ in Exp2 is different from those in Exp1 and Exp3.
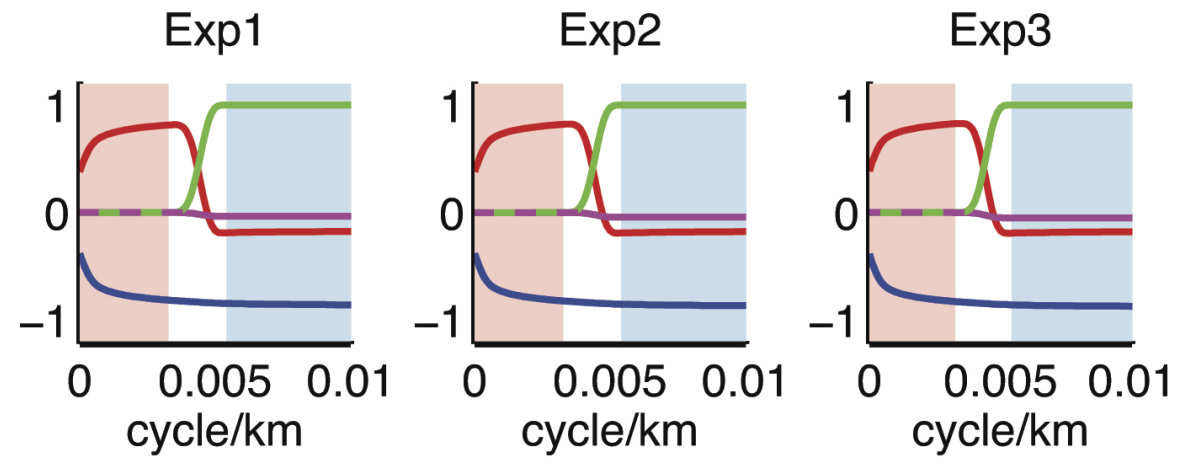

Figure 5-6: The normalized integral energy budget in the wavenumber domain in each experiment. Red curves denote $\left\langle\bar{\Pi}_{K}>\right.$, green curves denote $\left\langle\overline{\mathcal{F}}_{K}>\right.$, blue curves denote $<\bar{D}_{K}>$, and magenta curves denote $\overline{\frac{\partial}{\partial t}<K E_{K}^{<}>}$.

In our experiments, the energy cascade in the wavenumber domain does not depend on the mean flow (Figure 5-6). Positive (negative) slope of $<\overline{\Pi_{K}}>$ at the 

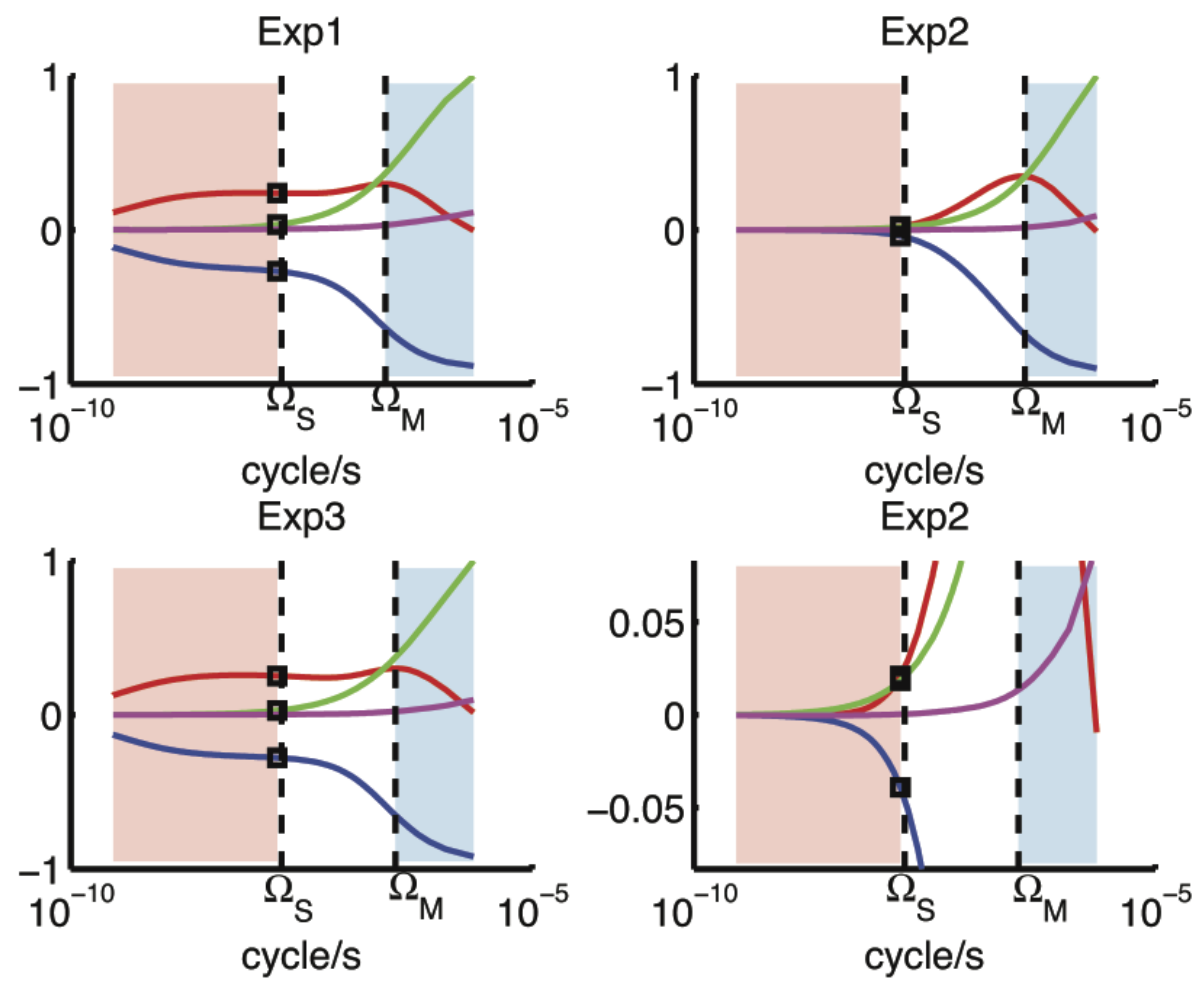

Figure 5-7: The normalized integral energy budget in the frequency domain in each experiments. Red curves denote $<\bar{\Pi}_{\Omega}>$. Green and blue curves denote respectively $<\overline{\mathcal{F}}_{\Omega}>$ and $\left\langle\bar{D}_{\Omega}>\right.$. $\overline{\frac{\partial}{\partial t}<K E_{\Omega}^{<}>}$is given by the magenta curve. Dashed lines in black are at $\Omega_{S}$ and the frequency with maximum $<\bar{\Pi}_{\Omega}>\left(\Omega_{M}\right)$. Black boxes are used to highlight the magnitude of each term at the frequency $\Omega_{S}$. The lower right panel is an enlarged version of the upper right panel to highlight budgets at frequencies lower than $\Omega_{S}$.

wavenumber $K$ means that eddies at that wavenumber gain (lose) energy through eddy-eddy interaction (Scott and Wang, 2005). The slope of $<\overline{\Pi_{K}}>$ is negative in the wavenumber range indicated by the white area, but it is positive in the range indicated by the red area (Figure 5-6). Thus, in all the experiments, energy moves to motions at low wavenumbers through eddy-eddy interaction.

In our experiments, $<\overline{\Pi_{\Omega}}>$ is sensitive to the meridional component of the mean flow (Figure 5-7). Similar to $<\overline{\Pi_{K}}>$, positive (negative) slope of $<\overline{\Pi_{\Omega}}>$ at the frequency $\Omega$ means that eddies at that frequency gain (lose) energy through eddy-eddy 
interaction. In Exp1 and Exp3, the slope of $<\overline{\Pi_{\Omega}}>$ is negative at frequencies higher than $\Omega_{M},<\overline{\Pi_{\Omega}}>$ is approximately constant over the frequency range $\left[\Omega_{S}, \Omega_{M}\right]$, and the slope is positive at frequencies lower than $\Omega_{S}$. In Exp2, the slope of $<\overline{\Pi_{\Omega}}>$ is also negative at frequencies higher than $\Omega_{M}$, but at the frequency range $\left[\Omega_{S}, \Omega_{M}\right]$, $<\overline{\Pi_{\Omega}}>$ reduces from its maximum value at $\Omega_{M}$ to roughly zero at $\Omega_{S}$. Therefore, energy mostly flows to motions with frequencies lower than $\Omega_{S}$ in Exp1 and Exp3, but energy mostly flows to the frequency range $\left[\Omega_{S}, \Omega_{M}\right]$ in Exp2 (the diagrams shown in Figure 5-8 further illustrate this point). Using Eq. 5.6, we find that the frequency of Rhines jets in Exp1 and Exp3 is roughly zero, but the frequency of Rhines jets in Exp2 lies in $\left[\Omega_{S}, \Omega_{M}\right]$. Thus, in all the three runs, energy moves to the frequency range where Rhines jets lie in.
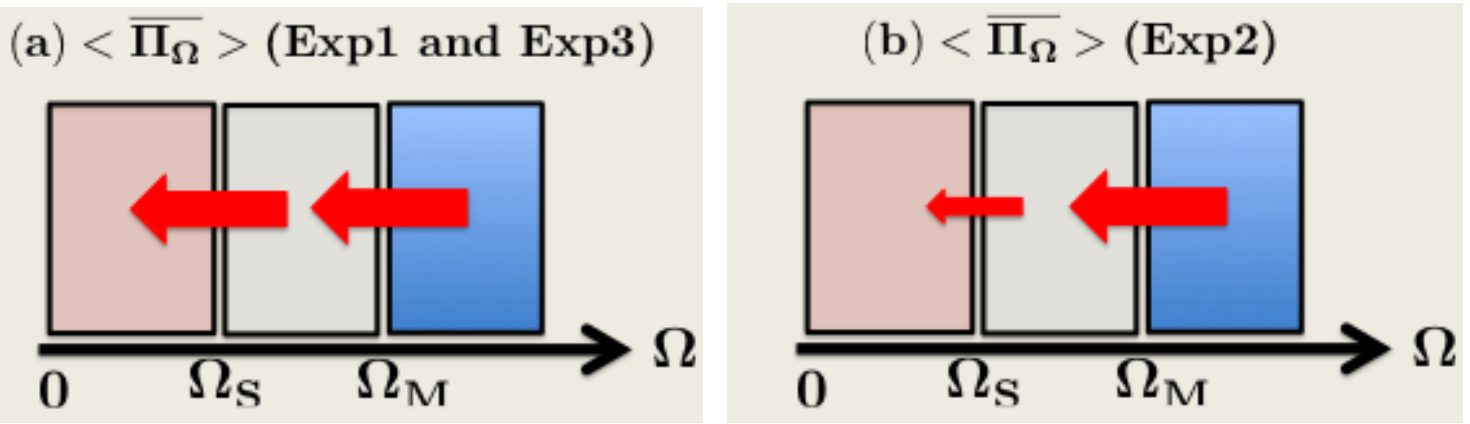

Figure 5-8: Diagrams illustrating the meaning of the $\left\langle\overline{\Pi_{\Omega}}>\right.$ structure shown in Figure 5-7. Black arrows at the bottom is the frequency axis $\Omega$; red arrows denote the nonlinear energy flux $<\overline{\Pi_{\Omega}}>$. In Exp1 and Exp3 (diagram a), the nonlinear energy flux goes through the $\Omega_{M}$ and $\Omega_{S}$ boundaries and is eventually used to sustain motions with frequencies lower than $\Omega_{S}$. In Exp2 (diagram b), the nonlinear flux through the $\Omega_{M}$ boundary is large, but little enters the $\Omega_{S}$ boundary. Thus, motions with frequencies lower than $\Omega_{S}$ get little energy through eddy-eddy interaction.

The above analysis suggests that the sensitivity of $<\overline{\Pi_{\Omega}}>$ to the imposed mean flow is because energy always moves to Rhines jets, whose frequency depends on the imposed mean flow. In the barotropic quasi-geostrophic system on a beta plane with no mean flow imposed, energy cascades to both low frequencies and low wavenum- 
bers; Rhines jets are low-frequency motions and extract energy from high-frequency motions (Rhines, 1975). Using the Galilean invariance of the barotropic system, we know that Rhines jets extract energy from other eddy motions regardless of the imposed mean flow. However, the frequency of Rhines jets is sensitive to the imposed meridional flow; thus, the structure of $<\overline{\Pi_{\Omega}}>$ is sensitive to the imposed meridional flow.

\section{Energy budget of striations}

The energy budget of striations is also sensitive to the imposed mean flow. The magnitude of the terms in striations' energy budget (Eq. 5.11) is highlighted by the black boxes in Figure 5-7. In Exp2, where Rhines jets are not part of striations, external forcing and eddy-eddy interaction are equally important energy sources of striations; but the amount of energy extracted by striations from other eddies is small. In Exp1 and Exp3, where striations are dominated by Rhines jets, the dominant energy source for striations is eddy-eddy interaction; the amount of energy extracted by striations from other eddies is large.

\subsection{Effect of mean flow on total mixing and stria- tions' contribution}

This section provides a diagnostic framework to partition the contribution of striations and high-frequency eddies to mixing, and presents a theory about the effect of mean flow on the spatially averaged mixing. We find that, in our experiments, mixing is always zonally dominant regardless of the imposed mean flow direction, and striations contribute significantly to zonal mixing only when striations are dominated by Rhines jets. 


\subsubsection{Diagnostic framework}

Eddies can induce tracer mixing, which is often quantified by estimating the effective diffusivity using either tracer-based or particle-based methods (e.g. Klocher et al., 2012b). To our best knowledge, though the effect of mean flow on tracer mixing

induced by the total eddies has been studied (e.g. Klocher et al., 2012a; Shuckburgh et al., 2009), the partition of the contribution of striations and high-frequency eddies to mixing has not been explored. Next we present one possible framework to split the contribution.

First, we describe how to parameterize the tracer mixing induced by total eddies. The ensemble average of the eddy tracer flux is

$$
\overline{F_{i}}=\overline{u_{i}^{\prime} b^{\prime}}
$$

where ${ }^{-}$is the ensemble average and $u_{i}^{\prime}$ is the eddy velocity. $b^{\prime}$ is the eddy tracer concentration satisfying

$$
\left[\frac{\partial}{\partial t}+\left(\overline{\mathbf{u}}+\mathbf{u}_{\mathbf{s}}^{\prime}+\mathbf{u}_{\mathbf{h}}^{\prime}\right) \cdot \nabla-\kappa \nabla^{2}\right] b^{\prime}=0
$$

where $\overline{\mathbf{u}}$ is the mean flow, $\mathbf{u}_{\mathbf{s}}^{\prime}$ is the low-frequency eddy velocity, and $\mathbf{u}_{\mathbf{h}}^{\prime}$ is the highfrequency eddy velocity. $\overline{F_{i}}$ can be parameterized as

$$
\overline{F_{i}}=-D_{i j} \frac{\partial \bar{b}(x, t)}{\partial x_{j}} .
$$

Diffusivity is the symmetric part of $D_{i j}$ and the transport due to the Stokes drift can be diagnosed from the antisymmetric part of $D_{i j}$. We use an unpublished formula 
from G. Flierl to calculate $D_{i j}$ :

$$
D_{i j}=\overline{\int u_{i}^{\prime}(x, t) U_{j}(x, t) d t}
$$

where $U_{j}(x, t)$ essentially records the initial velocity; it can be solved numerically by integrating

$$
\left[\frac{\partial}{\partial t}+\left(\overline{\mathbf{u}}+\mathbf{u}_{\mathbf{s}}^{\prime}+\mathbf{u}_{\mathbf{h}}^{\prime}\right) \cdot \nabla-\kappa \nabla^{2}\right] U_{j}(x, t)=0,
$$

with $U_{j}\left(x_{0}, t_{0}\right)=u_{j}^{\prime}\left(x_{0}, t_{0}\right)$ as the initial condition. Imagine the concentration of a tracer has the same magnitude as the eddy velocity at $t=t_{0}$ and then the tracer is stirred by the total flow field; $U_{j}(x, t)$ is just the concentration of the tracer at time $t$. $D_{i j}$ involves an ensemble average and thus it does not depend on the initial condition as long as enough ensembles are used in the calculation. These formulas (Eqs. 5.17 and 5.18) are based on the spatial homogeneity assumption. The appendix (Section 5.8.3) provides the details, and it also includes a formula without the homogeneity assumption (Eq. 5.58).

One simple way to split the contribution of striations and high-frequency eddies to mixing/transport is through the formula:

$$
\overline{F_{i}}=\overline{u_{i}^{\prime} b^{\prime}}=\overline{u_{h i}^{\prime} b^{\prime}}+\overline{u_{s i}^{\prime} b^{\prime}}=-\frac{\partial \bar{b}(x, t)}{\partial x_{j}} \overline{\int\left(u_{h i}^{\prime}+u_{s i}^{\prime}\right) U_{j}(x, t) d t},
$$

where $u_{s i}^{\prime}$ is the low-frequency eddy velocity and $u_{h i}^{\prime}$ is the high-frequency eddy velocity. Since striations account for more than $84 \%$ of the low-frequency motions in the three experiments (Section 5.4.1), it is reasonable to assume that all the low-frequency motions are striations. Thus $\overline{u_{s i}^{\prime} b^{\prime}}$ represents the contribution of striations to eddy tracer flux. Using a procedure similar to that in the appendix (Section 5.8.3), we can 
parameterize the two parts of the eddy tracer flux as

$$
\overline{u_{s i}^{\prime} b^{\prime}}=-D_{s i j} \frac{\partial}{\partial x_{j}} \bar{b}, \overline{u_{h i}^{\prime} b^{\prime}}=-D_{h i j} \frac{\partial}{\partial x_{j}} \bar{b}
$$

where

$$
D_{h i j}=\overline{\int u_{h i}^{\prime}(x, t) U_{j}(x, t) d t} \text { and } D_{s i j}=\overline{\int u_{s i}^{\prime}(x, t) U_{j}(x, t) d t} .
$$

$U_{j}$ here is exactly the same as the $U_{j}$ in the $D_{i j}$ formula (Eq. 5.18). The symmetric part of $D_{h i j}\left(D_{s i j}\right)$ represents the contribution of high-frequency eddies (striations) to mixing; the antisymmetric part of $D_{h i j}\left(D_{s i j}\right)$ provides information about the contribution of high-frequency eddies (striations) to the Stokes drift. Note that

$$
D_{h i j}+D_{s i j}=D_{i j}
$$

however, the tracer $b^{\prime}$ is advected by both the high-frequency eddy motions and striations (Eq. 5.15). Thus, the contribution of striations and high-frequency eddies to tracer mixing is not completely separable.

\subsubsection{Results}

Here are the specific four steps to obtain $\left\langle D_{s i j}\right\rangle$ and $\left\langle D_{h i j}\right\rangle$ shown in Table 5.3: (1) calculate $u_{h i}, u_{s i}$ and $U_{j}$, (2) integrate $u_{h i} U_{j}$ and $u_{s i} U_{j}$ over time t, (3) average them spatially, and (4) repeat the above three steps to get several ensembles and then ensemble average. We use a non-zero $\kappa\left(120 \mathrm{~km}^{2} /\right.$ day $)$ in order to make the integration $\int \cdot d t$ reach equilibrium in a reasonable amount of computation time. The calculation is computationally expensive, as it involves many ensembles and it involves calculating and saving the high-pass-filtered and low-pass-filtered eddy velocity at small time intervals. Thus, though $D_{i j}$ may be sensitive to the choice of 
$\kappa$ in Eq. 5.15, we did not explore this sensitivity problem in this study.

Table 5.3: The average of $\left\langle D_{h i j}\right\rangle$ and $\left\langle D_{s i j}>\right.$ over 30 independent ensembles $\left(10 \mathrm{~m}^{2} / \mathrm{s}\right)$, where $<\cdot>$ denotes the spatial average over the doubly-periodic domain. The error bar is $\sigma / \sqrt{N}$, where $\sigma$ is the standard deviation of $\left\langle D_{h i j}\right\rangle$ and $\left\langle D_{s i j}\right\rangle$. We choose $\mathrm{N}$, the number of degrees of freedom, to be $30 . D_{11}\left(D_{22}\right)$ denotes zonal (meridional) eddy diffusivity; $D_{12}$ and $D_{21}$ contain information about the Stokes drift.

\begin{tabular}{lrrrrrrrr}
\hline \hline & $D_{s 11}$ & $D_{h 11}$ & $D_{s 22}$ & $D_{h 22}$ & $D_{s 12}$ & $D_{h 12}$ & $D_{s 21}$ & $D_{h 21}$ \\
\hline Exp1 & $8198 \pm 534$ & $274 \pm 21$ & $27 \pm 1$ & $66 \pm 2$ & $20 \pm 14$ & $1 \pm 5$ & $1 \pm 3$ & $-5 \pm 3$ \\
Exp2 & $581 \pm 78$ & $7403 \pm 400$ & $28 \pm 1$ & $67 \pm 2$ & $-3 \pm 5$ & $8 \pm 15$ & $-37 \pm 5$ & $39 \pm 3$ \\
Exp3 & $7175 \pm 461$ & $336 \pm 23$ & $20 \pm 0$ & $76 \pm 1$ & $-1 \pm 12$ & $-3 \pm 5$ & $3 \pm 2$ & $-2 \pm 3$ \\
\hline
\end{tabular}

Though the mean flow can suppress mixing in some more complicated systems (e.g. Klocher et al., 2012a), the mean flow does not affect the domain averaged mixing in our barotropic system. In Exp1-Exp3, the magnitude of $\left\langle D_{i j}\right\rangle$, which is equal to $<D_{h i j}+D_{s i j}>$, does not differ much from each other (Table 5.3). This is related to the Galilean invariance: tracers and the eddy field are all advected by the mean flow. The appendix (Section 5.8.4) mathematically explains why the imposed mean flow does not affect the domain averaged mixing.

Consistently, mixing is anisotropic and mostly in the zonal direction regardless of the imposed mean flow: in all the three experiments, $\left\langle D_{11}\right\rangle$ has the largest magnitude among the four components of $\left\langle D_{i j}\right\rangle$ (Table 5.3). This phenomenon can be interpreted as follows: in the coordinates moving with the imposed mean flow, the eddy velocity is dominated by that of the Rhines jets, which are roughly zonal. Thus, most particles (parcels) are moving in the quasi-zonal direction and mixing is zonally dominant.

Rhines jets are always the dominant contributor to zonal mixing $\left.\left(<D_{11}\right\rangle\right)$ and thus striations contribute significantly to zonal mixing only when striations are dominated by Rhines jets. Table 5.3 shows the magnitude of $\left\langle D_{s i j}>\right.$ and $<D_{h i j}>$. In 
Exp1 and Exp3, where the meridional component of the imposed mean flow is zero, striations are dominated by Rhines jets and thus striations contribute much more to zonal mixing than the high-frequency eddies. In Exp2, where the meridional component of the imposed mean flow is not zero, Rhines jets are not part of the striations and thus striations contribute much less to zonal mixing than the high-frequency eddies.

\subsection{Applications}

\section{Diagnostic framework}

The key application of our work is that one can use our diagnostic framework and their potential descendants to investigate the contribution of striations to mixing and the energy cycle in realistic eddying ocean models, such as the ECCO2 state estimate. Considering the non-negligible contribution of striations to the oceanic variability, the contribution of striations to mixing and energy cycle is probably important (Chapter 4). Yet, little is known in this aspect.

Our framework in Section 5.5.1 is directly applicable to quantifying striations' role in tracer mixing in selected oceanic regions (local spatial homogeneity assumption is required), such as the CNP and ENP regions discussed in Chapter 4. Specifically, one could first identify the separation frequency between striations and high-frequency eddies using the approach proposed in Chapter 4, then calculate eddy diffusivity due to striations using our framework, and compare it with eddy diffusivity induced by total eddies. Work in this aspect can shed light on the role of striations in the spatial distribution of tracers (e.g. temperature, salinity) and thus the climate mean state and variability.

As to our diagnostic framework about striations' energy budgets (Section 5.4.1), 
it can be easily generalized to the form consistent with primitive equations. The generalized diagnostic framework can be used in the study of striation energetics in eddying ocean models. Compared to the framework in Thompson and Richards (2011), the advantage of our framework is that no spectrum-gap assumption is made.

\section{Striation interpretation}

The ease of interpretation about striations in the barotropic quasi-geostrophic model comes at a cost: different from the eddy-propagation mechanism proposed in Chapter 4, our barotropic striation interpretation (quasi-stationary barotropic Rossby waves) may not be directly applicable to most ocean regions. Rhines jets show up in the snapshots in our experiments, whereas banded structures are latent in most ocean regions (Thompson, 2010). Also the barotropic and the first baroclinic modes are coupled in most ocean regions (Wortham, 2012), but the coupling cannot be included in our barotropic model. In retrospect, we would further choose a multiplelayer model or the ECCO2 state estimate to investigate the consequences of striations to the oceanic circulation.

However, some kinematic aspects of our Exp2 in this chapter are analogous to the Southern Ocean jets. In contrast to most oceanic regions, banded structures are visible in the snapshot of the velocity field in the Southern Ocean and these structures are termed "Southern Ocean jets" (Thompson, 2010). These jets are generated from the mixing of potential vorticity by eddies from a kinematic perspective and they are greatly influenced by topography (e.g. Thompson, 2008; Ivchenko et al., 2008). For example, topography with the scale comparable to the jet width can cause the meridional drifts of jets in the quasi-geostrophic two-layer model (Thompson, 2010). Thompson and Richards (2011) found that meridional drifts of the jets also exist in some region of the Southern Ocean in realistic eddy-permitting models. Our Exp2 is analogous to some Southern Ocean jets in two kinematic aspects: banded structures 
are visiable in the instantaneous images of the flow field and they have meridional drifts. ${ }^{5}$ Results from Exp2 suggest that striations in the Southern Ocean might be different from the jets visible in the flow snapshots. The validity of the inference needs to be tested.

\subsection{Conclusions}

This chapter presents diagnostic framework about striations' contribution to mixing and energy pathway, and presents the effect of mean flow on the origin and consequences of barotropic striations. In our barotropic quasi-geostrophic system on a beta plane, energy is mostly concentrated on the surface of barotropic Rossby wave dispersion relation in the frequency and wavenumber space. Thus, striations can be viewed as quasi-stationary barotropic Rossby waves. The eddy-propagation mechanism identified from Chapter 4 does not apply here. In our experiments with no meridional mean flow, Rhines jets are low-frequency motions; striations are dominated by Rhines jets, contribute significantly to tracer mixing, and extract a signifiant amount of energy from high-frequency eddies. In our experiment with meridional mean flow, Rhines jets move meridionally and are not part of low-frequency motions; striations are tilted, have weak amplitude, contribute little to tracer mixing, and extract little energy from high-frequency eddies.

Our results can be useful in future explorations about striations and energetics. First, our diagnostic framework and the potential descendants can be useful in studies about the consequences of striations in realistic ocean systems. Second, our Exp2 has kinematic analogy to the Southern Ocean jets in some regions: bands are visible in the snapshots and they drift meridionally. Third, using the Galilean invariance

\footnotetext{
${ }^{5}$ The dynamical reasons for these two features in Exp2 are probably different from those in the Southern Ocean.
} 
of the barotropic model, we formulated theories illustrating why the mean flow does not influence the spatially averaged mixing and energy cascades in the wavenumber domain, but does influence the energy cascades in the frequency domain. Though these theories are only valid in our barotropic system, these simplifications may stimulate interest and further investigation about mean flow's effect on energy cascades in observations and realistic models. Work in this aspect might be useful in explaining the energy cascade features identified in Arbic et al. (2012).

Here we offer some critiques and caveats about this chapter. First, striations are banded structures in the low-frequency motions (Chapter 4), and one issue is to determine the separation frequency between low-frequency and high-frequency motions $\left(\Omega_{S}\right)$. The criterion to determine $\Omega_{S}$ presented in Chapter 4 does not apply well in idealized models and our choice of $\Omega_{S}$ here put Rhines jets in Exp2 into high-frequency motions. Though our striation interpretation (quasi-stationary Rossby waves) is not sensitive to $\Omega_{S}$, results about striations' energy budget and striations' contribution to mixing depend on $\Omega_{S}$. A more general criterion to determine $\Omega_{S}$ needs to be developed. Second, motivated by the fact that striations dominate the low-frequency motions in our three experiments, we assume all the low-frequency motions are striations, when we formulate our diagnostic framework about striations' role in tracer mixing and energy budget. However, only the banded structures in the low-frequency motions are defined as striations (Chapter 4). Yet, it is easy to generalize our diagnostic framework to the version suitable for evaluating the consequences of only the banded structures in low-frequency motions. Third, in our barotropic system, the dependence of our results on other parameters, such as the mean flow magnitude, bottom friction, the memory coefficient and the spectra of the external forcing, is still uncertain. Fourth, different from the eddy propagation mechanism identified in Chapter 4, our results here may not be directly applicable to most ocean regions due 
to the oversimplification of our model.

\subsection{Appendix}

\subsubsection{Diagnosis method for energy cascades}

Here we derive the diagnostic equations for temporal and spatial energy cascades. Since our goal is to investigate the energy pathway in the barotropic runs (Exp1, Exp2 and Exp3). We start the derivation from the barotropic potential vorticity equation:

$$
\frac{\partial}{\partial t} \nabla^{2} \psi+J\left(\psi, \nabla^{2} \psi\right)+J\left(-\bar{U} y+\bar{V} x, \nabla^{2} \psi\right)+\beta \frac{\partial}{\partial x} \psi=\mathcal{F}(x, y, t)-r \nabla^{2} \psi
$$

The variables in the above equation are defined in Section 5.2. First, we express the eddy stream function $\psi(x, y, t)$ by its Fourier series

$$
\psi(x, y, t)=\int \hat{\psi}(k, l, \omega) e^{i(k x+l y-\omega t)} d k d l d \omega
$$

Then we can define the spatially low-pass-filtered $\psi$ and the temporally low-passfiltered $\psi$ :

$$
\begin{aligned}
& \psi_{K}^{<}(x, y, t)=\int \hat{a}(k, l, \omega) e^{i(k x+l y-\omega t)} H_{1}(k, l, K) d k d l d \omega \\
& \psi_{\Omega}^{<}(x, y, t)=\int \hat{a}(k, l, \omega) e^{i(k x+l y-\omega t)} H_{2}(\omega, \Omega) d k d l d \omega
\end{aligned}
$$

where $H_{1}$ and $H_{2}$ are the low-pass filter, such as the box-car filter:

$$
H_{1}(k, l, K)=\frac{\sin (\pi k / K)}{\pi k / K} \frac{\sin (\pi l / K)}{\pi l / K}
$$




$$
H_{2}(\omega, \Omega)=\frac{\sin (\pi \omega / \Omega)}{\pi \omega / \Omega} .
$$

Thus, $\psi_{K}^{<}(x, y, t)$ represents eddy motions with wavenumbers smaller than $K$ and $\psi_{\Omega}^{<}(x, y, t)$ represents eddy motions with frequencies lower than $\Omega$. Then we obtain the domain averaged kinetic energy density from the low-wavenumber motions:

$$
<K E_{K}^{<}>=<\frac{1}{2}\left(\nabla \psi_{K}^{<} \cdot \nabla \psi_{K}^{<}\right)>
$$

and the domain averaged kinetic energy density from the low-frequency motions:

$$
<K E_{\Omega}^{<}>=<\frac{1}{2}\left(\nabla \psi_{\Omega}^{<} \cdot \nabla \psi_{\Omega}^{<}\right)>
$$

where $<\cdot>$ means the spatial average in the doubly-periodic domain. Here are some properties of the low-pass filter, which are used in the remaining derivation in this appendix:

$$
\left(\frac{\partial}{\partial t} \nabla^{2} \psi\right)_{K}^{<}=\frac{\partial}{\partial t} \nabla^{2} \psi_{K}^{<} \text {and }\left(\frac{\partial}{\partial t} \nabla^{2} \psi\right)_{\Omega}^{<}=\frac{\partial}{\partial t} \nabla^{2} \psi_{\Omega}^{<}
$$

Also in the doubly-periodic domain, variables are periodic and thus the following relations hold:

$$
\begin{gathered}
<-\psi \frac{\partial}{\partial t} \nabla^{2} \psi>=\frac{1}{2} \frac{\partial}{\partial t}\langle\nabla \psi \cdot \nabla \psi> \\
<\beta \psi \frac{\partial}{\partial x} \psi>=0, \quad<\psi \frac{\partial}{\partial x} \nabla^{2} \psi>=0 \text { and }\left\langle\psi J\left(\psi, \nabla^{2} \psi\right)>=0 .\right.
\end{gathered}
$$

To obtain the evolution equation of $\left\langle K E_{K}^{<}\right\rangle$, first we apply the spatially lowpass filter to Eq. 5.20 and then multiply the low-pass-filtered Eq. 5.20 by $-\psi_{K}^{<}$. Using the properties described above, we obtain:

$$
\frac{\partial}{\partial t}<K E_{K}^{<}>=<\Pi_{K}>+<\mathcal{F}_{K}>+<D_{K}>
$$


with

$$
\Pi_{K}=\psi_{K}^{<} \cdot\left[J\left(\psi, \nabla^{2} \psi\right)\right]_{K}^{<}
$$

representing the energy flux to the $K E_{K}^{<}$reservoir due to eddy-eddy interaction;

$$
\mathcal{F}_{K}=-\psi_{K}^{<} \cdot[\mathcal{F}(x, y, t)]_{K}^{<}
$$

representing the energy input into the $K E_{K}^{<}$reservoir from the external forcing; and

$$
<D_{K}>=<-\psi_{K}^{<} \cdot\left[-r \nabla^{2} \psi\right]_{K}^{<}>=<r \cdot \psi_{K}^{<} \cdot \nabla^{2} \psi_{K}^{<}>=-r \cdot K E_{K}^{<}
$$

representing the sink of $K E_{K}^{<}$due to friction. Note that Eq. 5.25 is essentially the one-layer version of the energy cascades equation for the stratified quasi-geostrophic motions presented in Scott and Wang (2005).

We can obtain the evolution equation for $\psi_{\Omega}^{<}$using the similar approach. Multiply the temporally low-pass-filtered potential vorticity equation by $\psi_{\Omega}^{<}$, then we find

$$
\frac{\partial}{\partial t}<K E_{\Omega}^{<}>=<\Pi_{\Omega}>+<\mathcal{F}_{\Omega}>+<D_{\Omega}>
$$

with

$$
\Pi_{\Omega}=\psi_{\Omega}^{<} \cdot\left[J\left(\psi, \nabla^{2} \psi\right)\right]_{\Omega}^{<}
$$

representing the energy flux to the $K E_{\Omega}^{<}$reservoir due to eddy-eddy interaction;

$$
\mathcal{F}_{\Omega}=-\psi_{\Omega}^{<} \cdot[\mathcal{F}(x, y, t)]_{\Omega}^{<}
$$


representing the energy input into the $K E_{\Omega}^{<}$reservoir from the external forcing; and

$$
<D_{\Omega}>=<-\psi_{\Omega}^{<} \cdot\left[-r \nabla^{2} \psi\right]_{\Omega}^{<}>=<r \psi_{\Omega}^{<} \cdot \nabla^{2} \psi_{\Omega}^{<}>=-r \cdot K E_{\Omega}^{<}
$$

representing the sink of $K E_{\Omega}^{<}$due to friction.

In a system that reaches statistical equilibrium, $\frac{\partial}{\partial t}<\overline{K E_{K}^{<}}>$is approximately zero. Thus, the forcing term and nonlinear flux term should balance the dissipation term:

$$
\frac{\partial}{\partial t}<\overline{K E_{K}^{<}}>=<\overline{\Pi_{K}}>+<\overline{\mathcal{F}_{K}}>+<\overline{D_{K}}>\approx 0,
$$

where ${ }^{-}$means the long-term temporal average. Similarly, $\frac{\partial}{\partial t}<\overline{K E_{\Omega}^{<}}>$should be small for stationary processes and thus

$$
\frac{\partial}{\partial t}<\overline{K E_{\Omega}^{<}}>=<\overline{\Pi_{\Omega}}>+<\overline{\mathcal{F}_{\Omega}}>+<\overline{D_{\Omega}}>\approx 0 .
$$

\subsubsection{A theory about effect of mean flow on energy cascades}

We developed a theory to explain why the mean flow does not influence the spatial energy cascades, but does influence the temporal energy cascades. Basically, we convert the nonlinear energy flux terms $<\bar{\Pi}_{\Omega}>$ and $<\bar{\Pi}_{K}>$ into the frequencywavenumber form.

\section{Background}

As discussed in Section 5.3.2, assuming there is no external forcing or the external forcing is white noise or close to white noise (the forcing has zero or small decorrelation time scale), if $\phi_{A}(x, y, t)$ is the eddy stream function in the experiment with no mean flow, then

$$
\phi_{B}(x, y, t)=\phi_{A}(x-\bar{U} t, y-\bar{V} t, t)
$$


has the same statistical characteristics as $\psi(x, y, t)$, which refers to the eddy stream function in the experiment with mean flow. Thus, the nonlinear energy flux in the experiments with mean flow $\left(\Pi_{\Omega}\right)$ can be represented as a function of $\phi_{B}(x, y, t)$ :

$$
\begin{gathered}
\Pi_{\Omega}=[\psi(x, y, t)]_{\Omega}^{<} \cdot[J(x, y, t)]_{\Omega}^{<}=\Pi_{B, \Omega}=\left[\phi_{B}(x, y, t)\right]_{\Omega}^{<} \cdot\left[J_{B}(x, y, t)\right]_{\Omega}^{<} \\
\Pi_{K}=[\psi(x, y, t)]_{K}^{<} \cdot[J(x, y, t)]_{K}^{<}=\Pi_{B, K}=\left[\phi_{B}(x, y, t)\right]_{K}^{<} \cdot\left[J_{B}(x, y, t)\right]_{K}^{<},
\end{gathered}
$$

where

$$
\begin{gathered}
J_{B}(x, y, t)=J\left(\phi_{B}(x, y, t), \nabla^{2} \phi_{B}(x, y, t)\right), \\
J(x, y, t)=J\left(\psi(x, y, t), \nabla^{2} \psi(x, y, t)\right),
\end{gathered}
$$

$a_{\Omega}^{<}$is the part of a with frequencies smaller than $\Omega$, and $a_{K}^{<}$is the part of a with wavenumbers smaller than $K$. Note that

$$
J_{B}(x, y, t)=J_{A}(x-\bar{U} t, y-\bar{V} t, t)
$$

where $J_{A}(x, y, t)=J\left(\phi_{A}(x, y, t), \nabla^{2} \phi_{A}(x, y, t)\right)$. Also note that

$$
\widehat{\phi_{B}}(k, l, \omega)=\widehat{\phi_{A}}(k, l, \omega-k \bar{U}-l \bar{V}) \text { and } \widehat{J_{B}}(k, l, \omega)=\widehat{J_{A}}(k, l, \omega-k \bar{U}-l \bar{V}) \text {, }
$$

where

$$
\widehat{a}(k, l, \omega)=\iiint a(x, y, t) e^{-i(k x+l y-\omega t)} d x d y d t .
$$

Next, we discuss the low-pass filter in the wavenumber or frequency domain. Take the box-car filter as an example, the low-pass wavenumber filter is

$$
H_{1}(k, l, K)=\frac{\sin (\pi k / K)}{\pi k / K} \frac{\sin (\pi l / K)}{\pi l / K}
$$


and the low-pass frequency filter is

$$
H_{2}(\omega, \Omega)=\frac{\sin (\pi \omega / \Omega)}{\pi \omega / \Omega} .
$$

Using the definition of filters (Eqs. 5.35 and 5.36), Eq. 5.29, Eq. 5.32, and Eq. 5.33, we can get the following relations:

$$
\begin{gathered}
\widehat{\phi_{B, K}^{<}}=\widehat{\phi_{B}}(k, l, \omega) \cdot H_{1}(k, l, K)=\widehat{\phi_{A}}(k, l, \omega-k \bar{U}-l \bar{V}) \cdot H_{1}(k, l, K) \\
\widehat{J_{B, K}^{<}}=\widehat{J_{B}}(k, l, \omega) \cdot H_{1}(k, l, K)=\widehat{J_{A}}(k, l, \omega-k \bar{U}-l \bar{V}) \cdot H_{1}(k, l, K) \\
\widehat{\phi_{B, \Omega}^{<}}=\widehat{\phi_{B}}(k, l, \omega) \cdot H_{2}(\omega, \Omega)=\widehat{\phi_{A}}(k, l, \omega-k \bar{U}-l \bar{V}) \cdot H_{2}(\omega, \Omega) \\
\widehat{J_{B, \Omega}^{<}}=\widehat{J_{B}}(k, l, \omega) \cdot H_{2}(\omega, \Omega)=\widehat{J_{A}}(k, l, \omega-k \bar{U}-l \bar{V}) \cdot H_{2}(\omega, \Omega) .
\end{gathered}
$$

An important tool used in this problem is the 3-dimensional convolution theorem: the Fourier transform of $f(x, y, t) g(x, y, t)$ is the convolution of the Fourier transform of $f(x, y, t)$ and $g(x, y, t)$. That is,

$$
\widehat{f g}(k, l, \omega)=\int \widehat{f}\left(k^{\prime}, l^{\prime}, \omega^{\prime}\right) \widehat{g}\left(k-k^{\prime}, l-l^{\prime}, \omega-\omega^{\prime}\right) d k^{\prime} d l^{\prime} d \omega^{\prime}
$$

Also note that the definition of the Fourier transform tells us that

$$
<\overline{f(x, y, t) g(x, y, t)}>=\left.\widehat{f g}(k, l, \omega)\right|_{k=0, l=0, \omega=0}
$$

where $\langle\cdot\rangle$ is the domain average and ${ }^{*}$ is the time average. Thus,

$$
<\overline{f(x, y, t) g(x, y, t)}>=\int \widehat{f}\left(k^{\prime}, l^{\prime}, \omega^{\prime}\right) \widehat{g}\left(-k^{\prime},-l^{\prime},-\omega^{\prime}\right) d k^{\prime} d l^{\prime} d \omega^{\prime}
$$


The one dimensional version of the formula (Eq. 5.43) has been used by Chinn and Gille (2007) to evaluate the effect of temporal sampling interval on eddy heat fluxes.

\section{Energy cascades in the wavenumber domain}

Next, we express the nonlinear flux $<\overline{\Pi_{K}(x, y, t)}$ as a function of the eddy field in the case with no mean flow $\left(\widehat{\phi}_{a}\right.$ and $\left.\widehat{J}_{a}\right)$. Using Eq. 5.31, Eq. 5.37, Eq. 5.38 and Eq. 5.43, we obtain

$$
\begin{aligned}
& \left.<\overline{\Pi_{K}(x, y, t}\right)>=<\overline{\left[\phi_{B}(x, y, t)\right]_{K}^{<} \cdot\left[J_{B}(x, y, t)\right]_{K}^{<}}> \\
= & \int \widehat{\phi_{B}}\left(k^{\prime}, l^{\prime}, \omega^{\prime}\right) \cdot \widehat{J_{B}}\left(-k^{\prime},-l^{\prime},-\omega^{\prime}\right) \cdot H_{1}^{2}\left(k^{\prime}, l^{\prime}, K\right) d k^{\prime} d l^{\prime} d \omega^{\prime} \\
= & \int \widehat{\phi_{A}}\left(k^{\prime}, l^{\prime}, \omega^{\prime}-k^{\prime} \bar{U}-l^{\prime} \bar{V}\right) \cdot \widehat{J_{A}}\left(-k^{\prime},-l^{\prime},-\omega^{\prime}+k^{\prime} \bar{U}+l^{\prime} \bar{V}\right) \cdot H_{1}^{2}\left(k^{\prime}, l^{\prime}, K\right) d k^{\prime} d l^{\prime} d \omega^{\prime}
\end{aligned}
$$

Define $\omega_{0}=\omega^{\prime}-k^{\prime} \bar{U}-l^{\prime} \bar{V}$, we can convert Eq. 5.44 to the following form

$$
<\overline{\Pi_{K}(x, y, t)}>=\int\left[\int_{-\infty-k^{\prime} \bar{U}-l^{\prime} \bar{V}}^{\infty-k^{\prime} \bar{U}-l^{\prime} \bar{V}} \widehat{\phi_{A}}\left(k^{\prime}, l^{\prime}, \omega_{0}\right) \widehat{J_{A}}\left(-k^{\prime},-l^{\prime},-\omega_{0}\right) d \omega_{0}\right] H_{1}^{2}\left(k^{\prime}, l^{\prime}, K\right) d k^{\prime} d l^{\prime} .
$$

That is,

$$
<\overline{\Pi_{K}(x, y, t)}>=\int \widehat{\left[\phi_{A}\right.}(k, l, \omega) \widehat{J_{A}}(-k,-l,-\omega) H_{1}^{2}(k, l, K) d k d l d \omega .
$$

Therefore, the nonlinear energy flux in the wavenumber domain $\left.\left(<\overline{\Pi_{K_{S}}(x, y, t)}\right\rangle\right)$ is only a function of the eddy stream function in the case with no mean flow imposed. This suggests that the spatial energy cascade characteristics do not depend on the imposed mean flow $(\bar{U}, \bar{V})$. This is confirmed by the numerical analysis as described in the main text. 


\section{Energy cascades in the frequency domain}

Using the similar technique, we can also express the nonlinear energy flux as a

function of eddy stream function in the case with no mean flow $\left(\widehat{\phi_{A}}\right.$ and $\left.\widehat{J_{A}}\right)$. Using Eq. 5.30, Eq. 5.39, Eq. 5.40 and Eq. 5.43, we obtain

$$
\begin{aligned}
& \left.<\overline{\Pi_{\Omega}(x, y, t}\right)>=<\overline{\left[\phi_{B}(x, y, t)\right]_{\Omega}^{<} \cdot\left[J_{B}(x, y, t)\right]_{\Omega}^{<}}> \\
= & \int \widehat{\phi_{B}}\left(k^{\prime}, l^{\prime}, \omega^{\prime}\right) \cdot \widehat{J_{B}}\left(-k^{\prime},-l^{\prime},-\omega^{\prime}\right) \cdot H_{2}^{2}\left(\omega^{\prime}, \Omega\right) d k^{\prime} d l^{\prime} d \omega^{\prime} \\
= & \int \widehat{\phi_{A}}\left(k^{\prime}, l^{\prime}, \omega^{\prime}-k^{\prime} \bar{U}-l^{\prime} \bar{V}\right) \cdot \widehat{J_{A}}\left(-k^{\prime},-l^{\prime},-\omega^{\prime}+k^{\prime} \bar{U}+l^{\prime} \bar{V}\right) \cdot H_{2}^{2}\left(\omega^{\prime}, \Omega\right) d k^{\prime} d l^{\prime} d \omega^{\prime} .
\end{aligned}
$$

If we define $\omega_{0}=\omega^{\prime}-k^{\prime} \bar{U}-l^{\prime} \bar{V}$, we obtain

$$
<\overline{\Pi_{\Omega}(x, y, t)}>=\int \widehat{\phi_{A}}\left(k^{\prime}, l^{\prime}, \omega_{0}\right) \cdot \widehat{J_{A}}\left(-k^{\prime},-l^{\prime},-\omega_{0}\right) \cdot H_{2}^{2}\left(\omega_{0}+k^{\prime} \bar{U}+l^{\prime} \bar{V}, \Omega\right) d k^{\prime} d l^{\prime} d \omega_{0} .
$$

That is,

$$
<\overline{\Pi_{\Omega}(x, y, t)}>=\int \widehat{\phi_{A}}(k, l, \omega) \cdot \widehat{J_{A}}(-k,-l,-\omega) \cdot H_{2}^{2}(\omega+k \bar{U}+l \bar{V}, \Omega) d k d l d \omega .
$$

As we change the imposed mean flow $(\bar{U}, \bar{V})$, essentially the low-pass filter $H_{2}$ in Eq. 5.49 picks up different part of $\widehat{\phi_{A}}(k, l, \omega) \cdot \widehat{J_{A}}(-k,-l,-\omega)$ in the frequency domain. Thus, the nonlinear energy flux in the frequency domain depends on the imposed mean flow. Our numerical analysis in the main text confirms the sensitivity of the temporal energy cascade characteristics to the imposed mean flow.

Arbic et al. (2012) also presents a formula about the nonlinear energy flux in the 
frequency domain. The barotropic version of their formula is

$$
\begin{aligned}
\left.<\overline{\Pi_{\Omega}(x, y, t}\right)> & =<\overline{[\psi(x, y, t)]_{\Omega}^{<} \cdot[J(x, y, t)]_{\Omega}^{<}}> \\
& =\int_{|\omega<\Omega|} \widehat{\phi}(k, l, \omega) \cdot \widehat{J}(-k,-l,-\omega) d k d l d \omega .
\end{aligned}
$$

Therefore, their formula essentially uses a rectangular filter, whereas our formula is derived in a more rigorous way and it is more general allowing us to use any type of filter $\left(H_{2}\right)$. The effect of mean flow on $<\overline{\Pi_{\Omega}}>$ (Eq. 5.48) is also not discussed in their work.

\subsubsection{Diagnosis method for mixing and transport}

Eddy tracer flux in this chapter is parameterized using an unpublished method from G. Flierl. Here we describe the method in detail for the convenience of the reader.

\section{Solution of eddy tracer flux}

Assume the tracer equation is

$$
\frac{\partial b}{\partial t}+\mathbf{u} \cdot \nabla b=\kappa \nabla^{2} b,
$$

where $b$ is the tracer concentration. Decompose $b$ into two components (ensemble average and deviation): $b=\bar{b}+b^{\prime}$, substitute $b=\bar{b}+b^{\prime}$ into Eq. 5.51 and then ensemble average, we obtain

$$
\frac{\partial \bar{b}}{\partial t}+\overline{\mathbf{u}} \cdot \nabla \bar{b}+\overline{\mathbf{u}^{\prime} \cdot \nabla b^{\prime}}=\kappa \nabla^{2} \bar{b},
$$

where $\overline{\mathbf{u}}$ is the mean velocity and $\mathbf{u}^{\prime}$ is the eddy velocity. Then using the Boussinesq 
assumption, we obtain

$$
\frac{\partial \bar{b}}{\partial t}+\overline{\mathbf{u}} \cdot \nabla \bar{b}-\kappa \nabla^{2} \bar{b}=-\nabla \cdot \overline{\mathbf{F}}
$$

where $\mathbf{F}=\overline{\mathbf{u}^{\prime} b^{\prime}}$. Subtract Eq. 5.53 from Eq. 5.51, then we get the equation for $b^{\prime}$ :

$$
\frac{\partial b^{\prime}}{\partial t}+\left(\overline{\mathbf{u}}+\mathbf{u}^{\prime}\right) \cdot \nabla b^{\prime}-\kappa \nabla^{2} b^{\prime}=-u^{\prime} \cdot \nabla \bar{b}+\nabla \cdot \overline{\mathbf{F}}
$$

The goal here is to parameterize $\mathbf{F}$. First, assuming $\overline{\mathbf{u}}, \mathbf{u}^{\prime}$ and $\bar{b}$ are known, we solve $b^{\prime}$ in Eq. 5.54. Define the Green's function for the linear operator for $b^{\prime}$ on the left hand side of Eq. 5.54 as $G\left(x, t \mid x^{\prime}, t^{\prime}\right)$ :

$$
\left[\frac{\partial}{\partial t}+\left(\overline{\mathbf{u}}+\mathbf{u}^{\prime}\right) \cdot \nabla-\kappa \nabla^{2}\right] G\left(x, t \mid x^{\prime}, t^{\prime}\right)=\delta\left(x-x^{\prime}\right) \delta\left(t-t^{\prime}\right)
$$

Thus, the solution to Eq. 5.54 is

$$
b^{\prime}(x, t)=-\int d x^{\prime} d t^{\prime} G\left(x, t \mid x^{\prime}, t^{\prime}\right) u_{j}^{\prime}\left(x^{\prime}, t^{\prime}\right) \frac{\partial \bar{b}\left(x^{\prime}, t^{\prime}\right)}{\partial x_{j}^{\prime}}+\int d x^{\prime} d t^{\prime} G\left(x, t \mid x^{\prime}, t^{\prime}\right) \frac{\partial \overline{F_{j}}}{\partial x_{j}^{\prime}}
$$

and the eddy flux $\overline{F_{i}}$ is

$$
\begin{aligned}
& \overline{F_{i}}=\overline{u_{i}^{\prime} b^{\prime}}=-\int d x^{\prime} d t^{\prime} \overline{u_{i}^{\prime}(x, t) G\left(x, t \mid x^{\prime}, t^{\prime}\right) u_{j}^{\prime}\left(x^{\prime}, t^{\prime}\right)} \frac{\partial \bar{b}\left(x^{\prime}, t^{\prime}\right)}{\partial x_{j}^{\prime}} \\
& +\int d x^{\prime} d t^{\prime} \overline{u_{i}^{\prime} G\left(x, t \mid x^{\prime}, t^{\prime}\right)} \frac{\partial \overline{F_{j}}}{\partial x_{j}^{\prime}} .
\end{aligned}
$$

Now we can get

$$
\overline{F_{i}}=\overline{u_{i}^{\prime} b^{\prime}}=-\int d x^{\prime} d t^{\prime} \mathcal{K}_{i j}\left(x, t \mid x^{\prime}, t^{\prime}\right) \frac{\partial \bar{b}\left(x^{\prime}, t^{\prime}\right)}{\partial x_{j}^{\prime}}+\int d x^{\prime} d t^{\prime} \mathcal{H}_{i}\left(x, t \mid x^{\prime}, t^{\prime}\right) \frac{\partial \overline{F_{j}}}{\partial x_{j}^{\prime}}
$$


where

$$
\mathcal{K}_{i j}\left(x, t \mid x^{\prime}, t^{\prime}\right)=\overline{u_{i}^{\prime}(x, t) G\left(x, t \mid x^{\prime}, t^{\prime}\right) u_{j}^{\prime}\left(x^{\prime}, t^{\prime}\right)}
$$

and

$$
\mathcal{H}_{i}\left(x, t \mid x^{\prime}, t^{\prime}\right)=\overline{u_{i}^{\prime} G\left(x, t \mid x^{\prime}, t^{\prime}\right)} .
$$

Eq. 5.58 tells us that the eddy tracer flux at $(x, t)$ depends on both local and nonlocal mean tracer gradient, indicating that the eddy tracer flux is a nonlocal process. Note that the only assumption we used to obtain $\mathcal{K}_{i j}\left(x, t \mid x^{\prime}, t^{\prime}\right)$ and $\mathcal{H}_{i}\left(x, t \mid x^{\prime}, t^{\prime}\right)$ from Eq. 5.51 is the Boussinesq assumption and thus these formulas quite accurately capture the mixing properties of tracers. However, in practice, diagnosing $\mathcal{K}_{i j}\left(x, t \mid x^{\prime}, t^{\prime}\right)$ is challenging, as it involves the computation of Green function. Thus, for computation purpose, we next further simplify Eq. 5.58 by assuming that the system is homogeneous.

\section{Computation technique and interpretation}

First, we focus on solving the term with $K_{i j}$ in Eq. 5.58. Define the operator

$$
D=\frac{\partial}{\partial t}+\left(\overline{\mathbf{u}}+\mathbf{u}^{\prime}\right) \cdot \nabla-\kappa \nabla^{2} .
$$

Consider the problem

$$
D U_{i}(x, t)=0,
$$

with the initial condition

$$
U_{i}\left(x, t_{0}\right)=u_{i}^{\prime}\left(x, t_{0}\right)
$$

In another form, the problem can be written as one single equation:

$$
D U_{i}(x, t)=\int u_{i}^{\prime}\left(x^{\prime}, t_{0}\right) \delta\left(t-t_{0}\right) \delta\left(x-x^{\prime}\right) d x^{\prime} .
$$


Here we explain why Eq. 5.62 is equivalent to Eqs. 5.60 and 5.61. First,

$$
\int u_{i}^{\prime}\left(x^{\prime}, t_{0}\right) \delta\left(t-t_{0}\right) \delta\left(x-x^{\prime}\right) d x^{\prime}=u_{i}^{\prime}\left(x, t_{0}\right) \delta\left(t-t_{0}\right) .
$$

Thus, when $t>t_{0}$, we obtain $D U_{i}(x, t)=u_{i}^{\prime}\left(x, t_{0}\right) \delta\left(t-t_{0}\right)=0$, which is just Eq. 5.60. If we integrate Eq. 5.62 over an infinitesimal time interval $\left[t_{0}-\Delta t, t_{0}+\Delta t\right]$, we obtain

$$
\left[U_{i}\left(x, t_{0}+\Delta t\right)-U_{i}\left(x, t_{0}-\Delta t\right)\right]+\int_{t_{0}-\Delta t}^{t_{0}+\Delta t}\left[\left(\overline{\mathbf{u}}+\mathbf{u}^{\prime}\right) \cdot \nabla-\kappa \nabla^{2}\right] U_{i}(x, t) d t=u_{i}^{\prime}\left(x, t_{0}\right)
$$

Assuming $U_{i}(x, t)$ is discontinuous at $t=t_{0}$ and $U_{i}(x, t)=0$ when $t<t_{0}$, as $\Delta t \rightarrow 0$, the left hand side of Eq. 5.63 is reduced to $U_{i}\left(x, t_{0}^{+}\right)$; thus $U_{i}\left(x, t_{0}^{+}\right)=u_{i}^{\prime}\left(x, t_{0}\right)$, which is equivalent to Eq. 5.61.

Recall that

$$
D G\left(x, t \mid x^{\prime}, t_{0}\right)=\delta\left(x-x^{\prime}\right) \delta\left(t-t_{0}\right)
$$

Integrate Eq. 5.64 with respect to $x^{\prime}$, we can get

$$
\begin{aligned}
\int u_{i}^{\prime}\left(x^{\prime}, t_{0}\right) D G\left(x, t \mid x^{\prime}, t_{0}\right) & d x^{\prime}=D \int u_{i}^{\prime}\left(x^{\prime}, t_{0}\right) G\left(x, t \mid x^{\prime}, t_{0}\right) d x^{\prime} \\
= & \int u_{i}^{\prime}\left(x^{\prime}, t_{0}\right) \delta\left(t-t_{0}\right) \delta\left(x-x^{\prime}\right) d x^{\prime}
\end{aligned}
$$

Comparison between Eqs. 5.65 and 5.62 tells us that

$$
U_{i}(x, t)=\int u_{i}^{\prime}\left(x^{\prime}, t_{0}\right) G\left(x, t \mid x^{\prime}, t_{0}\right) d x^{\prime}
$$

Assuming the system is homogeneous, then $\nabla \cdot \bar{F}$ is not an important term in the equation of $b^{\prime}$ and thus the term with $H_{i}$ in Eq. 5.58 is negligible. Also assuming 
that there is a scale separation, that is, $\nabla \bar{b}$ is approximately a constant, we can get

$$
\overline{F_{i}}=\overline{u_{i}^{\prime} b^{\prime}}=-\frac{\partial \bar{b}(x, t)}{\partial x_{j}} \int d x^{\prime} d t^{\prime} \overline{u_{i}^{\prime}(x, t) G\left(x, t \mid x^{\prime}, t^{\prime}\right) u_{j}^{\prime}\left(x^{\prime}, t^{\prime}\right)}
$$

That is,

$$
\overline{F_{i}}=-\frac{\partial \bar{b}(x, t)}{\partial x_{j}} \overline{\int d t^{\prime} u_{i}^{\prime}(x, t) \int G\left(x, t \mid x^{\prime}, t^{\prime}\right) u_{j}^{\prime}\left(x^{\prime}, t^{\prime}\right) d x^{\prime}}
$$

That is,

$$
\overline{F_{i}}=-\frac{\partial \bar{b}(x, t)}{\partial x_{j}} \overline{\left.\int u_{i}^{\prime}(x, t) U_{j}(x, t)\right|_{t^{\prime}=t_{0}} d t^{\prime}}
$$

For stationary process, we can change $d t_{0}$ into $d t$ in the following integral:

$$
\overline{F_{i}}=-\frac{\partial \bar{b}(x, t)}{\partial x_{j}} \overline{\int u_{i}^{\prime}(x, t) U_{j}(x, t) d t_{0}}=-\frac{\partial \bar{b}(x, t)}{\partial x_{j}} \overline{\int u_{i}^{\prime}(x, t) U_{j}(x, t) d t} .
$$

Therefore,

$$
\overline{F_{i}}=-\frac{\partial \bar{b}(x, t)}{\partial x_{j}} \overline{\int u_{i}^{\prime}(x, t) U_{j}(x, t) d t}=-D_{i j} \frac{\partial \bar{b}(x, t)}{\partial x_{j}}
$$

where $D_{i j}=\overline{\int u_{i}^{\prime}(x, t) U_{j}(x, t) d t}$.

Now consider the meaning of $D_{i j}$. Substitute Eq. 5.71 into Eq. 5.53, we can get

$$
\frac{\partial \bar{b}}{\partial t}+\overline{\mathbf{u}} \cdot \nabla \bar{b}-\kappa \nabla^{2} \bar{b}=-\nabla \cdot \overline{\mathbf{F}}=\partial_{i}\left(D_{i j} \partial_{j} \bar{b}\right)
$$

Decompose $D_{i j}$ into two parts

$$
D_{i j}=L_{i j}+K_{i j}
$$

where $K_{i j}$ is the symmetric part and $L_{i j}$ is the antisymmetric part of $D_{i j}$. Therefore,

$$
\frac{\partial \bar{b}}{\partial t}+(\overline{\mathbf{u}}+\widehat{\mathbf{u}}) \cdot \nabla \bar{b}-\kappa \nabla^{2} \bar{b}=\partial_{i}\left(K_{i j} \partial_{j} \bar{b}\right)
$$


where $\widehat{\mathbf{u}}=-\partial_{i} L_{i j}$ representing the Stokes drift part. The right hand side represents the eddy diffusion part. $K_{i j}$ is eddy diffusivity. Note that $k \ll K_{i j}$.

To summarize, assuming the system is homogeneous and there is a scale separation between the ensemble average and the eddies, the ensemble average of the eddy tracer flux can be parameterized as

$$
\overline{F_{i}}=-D_{i j} \frac{\partial \bar{b}(x, t)}{\partial x_{j}}
$$

where $D_{i j}=\overline{\int u_{i}^{\prime}(x, t) U_{j}(x, t) d t}$. $U_{j}$ can be solved numerically by integrating $D U_{i}=0$, with $U_{i}\left(x_{0}, t_{0}\right)=u_{i}^{\prime}\left(x_{0}, t_{0}\right)$. The symmetric part of $D_{i j}$ is the eddy diffusivity $K_{i j}$.

\subsubsection{A theory about effect of mean flow on mixing}

Here we discuss why the imposed mean flow does not influence the spatially averaged $D_{i j}$, that is $\left\langle D_{i j}\right\rangle$, in the barotropic quasi-geostrophic system on a beta-plane forced by external forcing with small or zero decorrelation time scales.

First, consider $D_{i j}$ in a system with no mean flow imposed. The eddy velocity and $D_{i j}$ in this case are respectively $\mathbf{u}_{A}^{\prime}$ and $D_{A i j}$. From Section 5.8.3, we know that

$$
D_{A i j}=\overline{\int u_{A i}^{\prime}(x, y, t) U_{A j}(x, y, t) d t},
$$

where $U_{A j}(x, y, t)$ satisfies

$$
\left(\frac{\partial}{\partial t}+\mathbf{u}_{\mathbf{A}}^{\prime} \cdot \nabla-\kappa \nabla^{2}\right) U_{A j}(x, y, t)=0
$$

with

$$
\left.U_{A j}\right|_{t=0}=\left.u_{A j}^{\prime}(x, y, t)\right|_{t=0} .
$$


Next, consider $D_{i j}$ in a system with mean flow $\overline{\mathbf{U}}$ imposed. The eddy velocity and $D_{i j}$ in this case are respectively $\mathbf{u}_{B}^{\prime}$ and $D_{B i j}$. Again, we know from Section 5.8.3 that

$$
D_{B i j}=\overline{\int u_{B i}^{\prime}(x, y, t) U_{B j}(x, y, t) d t},
$$

where $U_{B j}(x, y, t)$ satisfies

$$
\left(\frac{\partial}{\partial t}+\overline{\mathbf{U}} \cdot \nabla+\mathbf{u}_{\mathbf{B}}^{\prime} \cdot \nabla-\kappa \nabla^{2}\right) U_{B j}(x, y, t)=0
$$

with

$$
\left.U_{B j}(x, y, t)\right|_{t=0}=\left.u_{B j}^{\prime}(x, y, t)\right|_{t=0} .
$$

As in Section 5.3.1, if we use the Galilean invariance for the barotropic model and define a moving coordinate $\left(x^{\prime}, y^{\prime}, t\right)$ :

$$
x^{\prime}=x-\bar{U} t, y^{\prime}=y-\bar{V} t, t^{\prime}=t,
$$

we get

$$
\mathbf{u}_{\mathbf{B}}^{\prime}(x, y, t)=\mathbf{u}_{\mathbf{A}}^{\prime}\left(x^{\prime}, y^{\prime}, t\right)=\mathbf{u}_{\mathbf{A}}^{\prime}(x-\bar{U} t, y-\bar{V} t, t)
$$

from a statistical point of view (Section 5.3.2). Using Eq. 5.80, we can write Eqs. 5.78 and 5.79 in the coordinates $\left(x^{\prime}, y^{\prime}, t^{\prime}\right)$ :

$$
\left(\frac{\partial}{\partial t^{\prime}}+\mathbf{u}_{\mathbf{A}}^{\prime}\left(x^{\prime}, y^{\prime}, t\right) \cdot \nabla-\kappa \nabla^{2}\right) U_{B j}=0
$$

with

$$
\left.U_{B j}\right|_{t=0}=\left.u_{B j}^{\prime}(x, y, t)\right|_{t=0}=\left.u_{A j}^{\prime}\left(x^{\prime}, y^{\prime}, t^{\prime}\right)\right|_{t=0} .
$$


The comparison between Eqs. 5.81 and 5.82 with Eqs. 5.75 and 5.76 shows that

$$
U_{B j}=U_{A j}\left(x^{\prime}, y^{\prime}, t^{\prime}\right)=U_{A j}(x-\bar{U} t, y-\bar{V} t, t) .
$$

Eqs. 5.80 and 5.83 tells us that

$$
u_{B i}^{\prime} U_{B j}=u_{A i}^{\prime}(x-\bar{U} t, y-\bar{V} t, t) U_{A j}(x-\bar{U} t, y-\bar{V} t, t) .
$$

Thus, at every time step in the doubly-periodic domain,

$$
u_{B i}^{\prime}(x, y, t) U_{B j}(x, y, t)
$$

has the same spatial pattern as

$$
u_{A i}^{\prime}(x, y, t) U_{A j}(x, y, t) .
$$

Therefore,

$$
<u_{A i}^{\prime}(x, y, t) U_{A j}(x, y, t)>=<u_{B i}^{\prime}(x, y, t) U_{B j}(x, y, t)>
$$

and

$$
<D_{A i j}>=<D_{B i j}>
$$

where $<\cdot>$ denotes the spatial average over the doubly-periodic domain. Therefore, the spatially averaged eddy diffusivity does not depend on the imposed mean flow. 


\section{Chapter 6}

\section{Conclusions}

This thesis mainly presents our research on two problems about oceanic eddies. Chapter 3 is about the eddy-mean flow interaction in the global ocean from the ECCO2 state estimate. Chapters 4 and 5 are about banded structures in the low-frequency eddy field: their amplitude, contribution to mixing and energy budgets, and the effect of time-mean circulation on their origin and properties. Next, we summarize our main findings and discuss some remaining questions.

\subsection{ECCO2 state estimate evaluation}

The ECCO2 state estimate is an eddy-permitting global simulation and it serves as an important tool in this thesis. Thus, as a preparation for our study about eddies, we describe the ECCO2 state estimate and explore its fidelity through modeldata comparison in Chapter 2. We find that the amplitude of eddies in the ECCO2 state estimate is consistent with observations, especially in the upper $2000 \mathrm{~m}$ of the mid- and low latitude regions. Besides the consistency with observations, another advantage of this simulation is that it is a free-forward run using the optimized control 
parameters, which are obtained by reducing the model-data misfit using the Green function method. Therefore, the solution is dynamically consistent and thus is a proper tool for budget diagnosis and dynamics exploration. This thesis illustrates the utility of the ECCO2 state estimate in our eddy studies.

To further improve and develop the ECCO2 state estimate, we need to compare many more aspects about the eddy field with observations. For example, the altimetric data has recently been used extensively to evaluate the lifetime, amplitude, rotation speed and length scale of vortices (e.g. Chelton et al., 2011), and the particle dispersion and tracer mixing rate induced by eddies (e.g. Rypina et al., 2012; Abernathey, 2012). It is still unknown how much the ECCO2 state estimate is consistent with those observed eddy properties.

To improve the state estimate, one should also go beyond the superficial comparison and assess the causes of the model-data misfit. We speculate that the relative larger misfit at high-latitudes and deep ocean could be due to a combination of lack of observations there, poor quality of available observations, inadequate horizontal resolution compared to the deformation radius, and inadequate vertical resolution. Some sensitivity experiments may help assess the main source of errors and provide guidance for further improvement of the state estimate.

\subsection{Eddy-mean flow interaction in the global ocean}

Chapter 3 is mainly a description about eddy-mean flow interaction in the global ocean from the energy perspective. To out best knowledge, this is the first global diagnosis of the complete set of eddy-mean flow interaction energy terms (i.e. those listed in Table 3.1). Eddy-mean flow interaction through eddy momentum fluxes is most active in the western boundary regions and the Southern Ocean, whereas the 
interaction through eddy density fluxes is most pronounced in the western boundary regions, the Southern Ocean and the subtropical gyres. One of our contributions is that we illustrate the value of our eddy-mean flow interaction diagram (Figure 3-1), which is modified from the original Lorenz energy diagram by adding the energy divergence terms through eddy-mean flow interaction. Using this diagram, we obtain one of our key findings in this chapter: eddy-mean flow interaction through eddy density fluxes is to some extent nonlocal in the Southern Ocean and the western boundary extension regions, but it is local in the selected subtropical gyre region. ${ }^{1}$

Our results are incomplete and come with caveats. First, our results about local and nonlocal eddy-mean flow interaction are certainly not final words, due to uncertainties in the ECCO2 state estimate, the limited record length, and the quasigeostrophic assumption in our APE definition. Second, we did not close these energy budgets, as some model variables (e.g. varying viscosity and high-frequency signals) are not available due to the large storage requirement for the output. To close the budget without increasing the storage cost, we suggest develop some codes for MITgcm (Marshall et al., 1997a,b) to produce and save these energy terms or closely related fluxes directly, as the model runs. Third, our description focuses on the energy perspective of eddy-mean flow interaction. The pathways of momentum, vorticity and enstrophy in the ECCO2 state estimate during eddy-mean flow interaction process are to be characterized and interpreted.

On the other hand, our work also points towards some key future directions. A common assumption used in previous theoretical studies about eddies, jets and instabilities is that the ocean patch is homogeneous (e.g. Arbic and Flierl, 2004; Venaille et al., 2011; Panetta, 1993; Thompson, 2010). Eddy-mean flow interaction is local under this assumption; however, our work indicates that eddy-mean flow interaction

\footnotetext{
${ }^{1}$ Local vs. nonlocal eddy-mean flow interaction is defined in Chapter 3.
} 
is non-local in some regions. A question thus arises: what are the consequences of this non-local nature of eddy-mean flow interaction on instabilities, jet dynamics, eddy characteristics and eddy's contribution to transport and mixing? As to the cause of this non-local nature, we only provide a superficial answer in this descriptive chapter: many ocean regions are inhomogeneous. Further work needs to be done in this aspect. More future directions are listed in Chapter 3.

\subsection{Striations in the time-mean circulation and their consequences}

\section{Amplitude and consequences of striations}

One of the key conclusions in Chapter 4 is that a noticeable percentage of zonal velocity variability is associated with striations. Specifically, the analysis using both the ECCO2 state estimate and the altimetric data shows that more than $10 \%$ (20\%) of the zonal velocity variability is contained in striations in the upper $1100 \mathrm{~m}$ in the East (Central) North Pacific region. To estimate the percentage, we define striations as banded structures in the low-frequency motions and we provide a method to determine the separation frequency $\Omega_{S}$ between low-frequency and high-frequency motions. One caveat is that our method to determine $\Omega_{S}$ does not work at the depth level where a large number of grid points represent land.

Motivated by the non-negligible percentage, we suspect that striations have nonnegligible contribution to tracer mixing/transport and energy cycle. Thus, in Chapter 5 , we formulate diagnostic framework to quantify the consequences of striations in these two aspects and use the barotropic quasi-geostrophic model on a beta plane to test the utility of our framework. We find that, the contribution of striations to tracer 
mixing and energy budget has interesting behavior even in such a highly simplified model: their contribution is sensitive to the imposed mean flow direction and only when striations are dominated by Rhines jets that they contribute significantly to mixing and the energy cycle. We also find that in such a simple system, the mean flow has no effect on the energy cascade in the wavenumber domain and the spatially averaged total mixing of passive tracers, but the mean flow does have an effect on the energy cascade characteristics in the frequency domain.

\section{Effect of time-mean circulation on striations' origin}

Besides the amplitude and consequences of striations, we also explore the origin of striations in Chapters 4 and 5. Specifically, we investigate the effect of the time-mean subtropical gyre on the origin and properties of striations. This study is motivated by the fact that both striations and the gyre-scale time-mean circulation are pervasive in the world ocean.

In Chapter 4, first we analyze striations in the ECCO2 state estimate. We find that striations in the ENP region are embedded in a southward gyre flow and they tilt towards the southwestward direction, while striations in the CNP region are embedded in a westward gyre flow and they are zonal. One key finding is that striations roughly align with the eddy-propagation direction in the CNP region and the southern part of the ENP region. We proposed a hypothesis: the gyre-flow contributes to the difference of the striation direction in these two regions.

Next, we test this hypothesis in Chapter 4 by formulating and solving an idealized 1.5-layer model, which explicitly includes the gyre flow. Since we define striations as banded structures in low-frequency motions, we interpret their origin and the gyre-effect from the frequency-wavenumber spectra of eddies. Striations do exist in the idealized model and their directions are qualitatively similar to those from the ECCO2 state estimate. In the linear limit, we derive the analytical solution of the 
spectra and find that striations are quasi-stationary linear Rossby waves. In this case, the gyre-flow influences the striation direction through influencing the zero Rossby wave frequency curve in the wavenumber space. In the nonlinear case, striations follow the eddy propagation direction. We formulate a simplified spectra model for non-dispersively propagating eddies, which is consistent with the numerical result. The spectra model suggests that striations arise as the temporal averaging effect of these non-dispersively propagating eddies. The consistency between the eddypropagation direction and the striation direction is related to the non-dispersive lines in the spectra. In this case, the gyre-flow influences the striation direction by affecting the eddy-propagation direction. For example, eddies in the East Region propagate to the southwestward direction due to the advection of the southward gyre flow and thus striations are titled.

As described above, the main purpose of Chapter 5 is to develop the diagnostic framework to evaluate striations' consequences. To help test the diagnostic framework through further interpretation, Chapter 5 also explores the effect of mean flow on the striation origin in this nonlinear barotropic model on a beta plane. We find that $90 \%$ of the large values on the three-dimensional frequency-wavenumber spectra occur roughly on the surface of Rossby wave dispersion relation. Therefore, the eddy field here is approximately a set of weakly interacting Rossby waves. One main result is that striations here are quasi-stationary Rossby waves and whether Rhines jets are part of the striations depend on the meridional component of the imposed mean flow. The eddy-propagation mechanism does not apply here.

\section{Remaining problems}

Here I offer a brief description about some remaining issues about striations' amplitude, consequences, origin and mean flow's effect. Readers can refer to Chapters 4 and 5 for more discussions about remaining issues and applications. 
First, we only estimated the percentage of zonal velocity variability contained in striations in two regions. In order to get a more complete quantitative estimate about the importance of striations in oceanic variability, one can extend our work to other areas in the global ocean and to other oceanic variables. Besides using the ECCO2 state estimate and the altimetric data, one can also obtain the percentage from the model spectra for many oceanic variables (e.g. three-dimensional velocity, density, temperature) presented in Wortham (2012). The comparison between the percentage estimate from the model spectra and those from observations and eddying models is a test of whether the striation amplitude in the model spectra is realistic. This comparison can be useful in further improving the model spectra, which have many applications as listed in Wortham (2012).

Another possible future direction is to estimate the consequences of striations in realistic contexts (e.g. their contribution to tracer transport, energy, momentum and vorticity pathways in the global ocean). One limitation of Chapter 5 is that our barotropic model is an oversimplified representation of the ocean and thus our results may not be directly applicable to the ocean. Using our striation definition and our diagnostic framework and their potential descendants, one can evaluate the role of striations in tracer mixing, transport and energy cycle in some important ocean regions, such as the region for the Diapycnal and Isopycnal Mixing Experiment in the Southern Ocean (DIMES, Gille et al., 2007). ${ }^{2}$ The Southern Ocean State Estimate (i.e. SOSE, Mazloff et al., 2010) is one of the models suitable for this investigation. Studies in this aspect may eventually help assess the role of striations in setting the climate mean state (e.g. temperature and salinity) and improve mixing representations in oceanic models. Some other interesting questions are how much

\footnotetext{
${ }^{2}$ Previous studies show that striations do exist in the Southern Ocean (e.g. Figure 1 in Maximenko et al., 2008).
} 
energy from the wind power input eventually transfers to striations and how much energy loss is through the dissipation of striations.

A third question is to what extent our interpretations about the origin of striations identified from our simple models are relevant to the ocean. Among the interpretations we proposed, the eddy-propagation mechanism is probably most relevant to striations in the eddying realistic models and the ocean. This mechanism may be relevant in the open ocean between a few degrees off the equator and 45 degrees. Readers can refer to Sections 4.5 and 5.6 for more discussion about the relevance of our interpretations to the ocean and possible methods for quantitative tests.

Fourth, our interpretations about striations' origin are certainly not final. Our interpretations are mostly based on either analytical frequency-wavenumber spectra or idealized spectra model. We need to understand what sets the spectra shape, such as the slope of the non-dispersive line in the 1.5-layer model and the sensitivity of the spectra shape to the magnitude of deformation radius. Also many interpretations about the striation origin in previous studies are dynamical, such as Rossby wave instabilities and nonlinear self-interaction of eigenmodes (e.g. O'Reilly et al., 2012; Berloff, 2005). It is still unknown whether these various mechanisms are different or essentially consistent, and how to interpret these previously proposed mechanisms from the spectral perspective. If these mechanisms are indeed different, which mechanism dominates in the ocean? If some of them are consistent, how to synthesize the consistent ones into a general theory about striations? More relevant questions are in Chapters 4 and 5 .

Finally, one key limitation of our striation study is that our 1.5-layer model and the barotropic model are the simplest possible ones. We choose these models because one can isolate key dynamical factors and thus gain insights more easily, and one can test the utility of the new diagnostic framework easily due to its computational 
efficiency. However, though our models are complex enough to produce some interesting relevant features, some aspects of our models are not representative of the ocean (e.g. the absence of topography and modal coupling). We suggest further investigations using a hierarchy of ocean models to assess the role of topography, mean flow, modal coupling, eastern boundary current, islands and surface forcing on the origin, direction, amplitude and consequences of striations. A proper choice of the more complicated models is the two-layer model, the utility of which in the mid-latitude vortex study and the Southern Ocean jets study have been suggested by Arbic and Flierl (2004) and Thompson (2010). Comparison between results from these models with those from the realistic ECCO2 state estimate can also be useful. 


\section{Bibliography}

Abernathey, R., 2012: Mixing by ocean eddies. Ph.D. thesis, MIT/WHOI, 175pp.

Adcroft, A., J.-M. Campin, C. Hill, and J. Marshall, 2004: Implementation of an atmosphere-ocean general circulation model on the expanded spherical cube. Mon. Wea. Rev., 132, 2845-2863.

Afanasyev, Y. D., S. O. Leary, P. B. Rhines, and E. G. Lindahl, 2011: On the origin of jets in the ocean. Geophys. Astrophys. Fluid Dyn., in press.

Arbic, B. K., 2000: Generation of mid-ocean eddies: the local baroclinic instability hypothesis. Ph.D. thesis, MIT/WHOI, 290pp.

Arbic, B. K. and G. R. Flierl, 2004: Baroclinically unstable geostrophic turbulence in the limits of strong and weak bottom Ekman friction: application to midocean eddies. J. Phys. Oceanogr., 34, 2257-2273.

Arbic, B. K., R. B. Scott, G. R. Flierl, A. J. Morten, J. G. Richman, and J. F. Shriver, 2012: Nonlinear cascade of surface oceanic geostrophic kinetic energy in the frequency domain. J. Phys. Oceanogr., 42, 1577-1600.

Arbic, B. K., et al., 2009: Estimates of bottom flows and bottom boundary layer dissipation of the oceanic general circulation from global high-resolution models. J. Geophys. Res., 114, C02 024.

Baldwin, M., P. B. Rhines, H.-P. Huang, and M. E. McIntyre, 2007: The jet-stream conundrum. Science, 315, 467-468.

Berloff, P., 2005: On rectification of randomly forced flows. J. Mar. Res., 63, 497-527.

Berloff, P., I. Kamenkovich, and J. Pedlosky, 2009: A mechanism of formation of multiple zonal jets in the oceans. J. Fluid Mech., 628, 395-425.

Berloff, P., S. Karabasov, J. T. Farrar, and I. Kamenkovich, 2011: On latency of multiple zonal jets in the oceans. J. Fluid Mech., 686, 534-567.

Boé, J., A. Hall, and X. Qu, 2009: Deep ocean heat uptake as a major source of spread in transient climate change simulations. Geophys. Res. Lett., 36, L22 701. 
Boland, E. J. D., A. F. Thompson, E. Shuckburgh, and P. H. Haynes, 2012: The formation of non-zonal jets over sloped topography. J. Phys. Oceanogr., 42, 16351651.

Brown, J. N. and A. V. Fedorov, 2010: How much energy is transferred from the winds to the thermocline on ENSO time scales? J. Climate, 23, 1563-1580.

Capet, X., J. C. McWilliams, M. J. Molemaker, and A. F. Shchepetkin, 2008: Mesoscale to submesoscale transition in the California Current System. Part II: frontal processes. J. Phys. Oceanogr., 38, 44-64.

Centurioni, L. R., J. C. Ohlmann, and P. P. Niiler, 2008: Permanent meanders in the California Current system. J. Phys. Oceanogr., 38, 1690-1710.

Charney, J. G., 1947: The dynamics of long waves in a baroclinic westerly current. J. Meteor., 4, 135-162.

Charney, J. G. and G. R. Flierl, 1981: Oceanic analogues of large-scale atmospheric motions. Evolution of Physical Oceanography, Scientific Surveys in Honor of Henry Stommel, The MIT Press, 504-549.

Chelton, D. B., M. G. Schlax, and R. M. Samelson, 2011: Global observations of nonlinear mesoscale eddies. Prog. Oceanog., 91, 167-216.

Chelton, D. B., M. G. Schlax, R. M. Samelson, and R. A. de Szoeke, 2007: Global observations of large oceanic eddies. Geophys. Res. Lett., 34, L15 606.

Chinn, B. S. and S. T. Gille, 2007: Estimating eddy heat flux from float data in the North Atlantic: The impact of temporal sampling interval. J. Atmos. Oceanic Technol., 24, 923-934.

Collier, M. and P. Durack, 2006: CSIRO netCDF version of the NODC World Ocean Atlas 2005.

Cox, M., 1987: An eddy-resolving numerical model of the ventilated thermocline: time dependence. J. Phys. Oceanogr., 17, 1044-1056.

Cronin, M. and D. R. Watts, 1996: Eddy-mean flow interaction in the Gulf Stream at $68^{\circ} \mathrm{W}$. Part I: eddy energetics. J. Phys. Oceanogr., 26, 2107-2131.

Cushman-Roisin, B., B. Tang, and E. P. Chassignet, 1990: Westward motion of mesoscale eddies. J. Phys. Oceanogr., 20, 758-768.

Danilov, S. and V. M. Gryanik, 2004: Barotropic beta-plane rurbulence in a regime with strong zonal jets revisited. J. Atmos. Sci., 61, 2283-2295. 
Danilov, S. and D. Gurarie, 2004: Scaling, spectra and zonal jets in beta-plane turbulence. Physics of Fluids, 16, 2592-2603.

Delworth, T. L., et al., 2012: Simulated climate and climate change in the GFDL CM2.5 high-resolution coupled climate model. J. Climate, 25, 2755-2781.

Dibarboure, G., O. Lauret, F. Mertz, V. Rosmorduc, and C. Maheu, 2009: SSALTO/DUACS user handbook: (M)SLA and (M)ADT near-real time and delayed time products.

Doos, K. and D. Webb, 1994: The Deacon cell and other meridional cells of the Southern Ocean. J. Phys. Oceanogr., 24, 429-442.

Dritschel, D. G. and M. E. McIntyre, 2008: Multiple jets as PV staircases: the Phillips effect and the resilience of eddy-transport barriers. J. Atmos. Sci., 65, 855-874.

Eady, E., 1949: Long waves and cyclone waves. Tellus, 1, 33-52.

Early, J. J., R. M. Samelson, and D. B. Chelton, 2011: The evolution and propagation of quasigeostrophic ocean eddies. J. Phys. Oceanogr., 41, 1535-1555.

Eden, C., R. J. Greatbatch, and J. Willebrand, 2007: A diagnosis of thickness fluxes in an eddy-resolving model. J. Phys. Oceanogr., 37, 727-742.

Farrell, B. F., 1982: Pulse asymptotics of the Charney baroclinic instability problem. J. Atmos. Sci., 39, 507-517.

Farrell, B. F. and P. J. Ioannou, 2007: Structure and spacing of jets in barotropic turbulence. J. Atmos. Sci., 64, 3652-3665.

Ferrari, R. and C. Wunsch, 2009: Ocean circulation kinetic energy-reservoirs, sources and sinks. Ann. Rev. of Fluid Mech., 41, 253-282.

Ferrari, R. and C. Wunsch, 2010: The distribution of eddy kinetic and potential energies in the global ocean. Tellus, 62A, 92-108.

Flierl, G. R., 1977: The application of linear quasigeostrophic dynamics to Gulf Stream rings. J. Phys. Oceanogr., 7, 365-379.

Flierl, G. R., 1984: Rossby wave radiation from a strongly nonlinear warm eddy. J. Phys. Oceanogr., 14, 47-58.

Flierl, G. R. and D. J. McGillicuddy, 2002: Mesoscale and submesoscale physicalbiological interactions. In Biological-physical interactions in the sea. The Sea, A. Robinson, J. J. McCarthy, and B. J. Rothschild, Eds., John Wiley and Sons, Inc., New York, Vol. 12, 113-185. 
Forget, G. and C. Wunsch, 2007: Estimated global hydrographic variability. J. Phys. Oceanogr., 37, 1997-2008.

Fox-Kemper, B., G. Danabasoglu, R. Ferrari, and R. W. Hallberg, 2008: Parameterizing submesoscale physics in global climate models. CLIVAR Exchanges, 13(1), $3-5$.

Fox-Kemper, B., G. Danabasoglu, R. Ferrari, and R. W. Hallberg, 2011: Parameterization of mixed layer eddies. III: Implementation and impact in global ocean climate simulations. Ocean Modelling, 39, 61-78.

Fox-Kemper, B., R. Ferrari, and J. Pedlosky, 2003: On the indeterminacy of rotational and divergent eddy fluxes. J. Phys. Oceanogr., 33, 478-483.

Fox-Kemper, B. and D. Menemenlis, 2008: Can large eddy simulation techniques improve mesoscale-rich ocean models? Ocean modeling in an eddying regime, Geophys. Monogr. Ser., M. W. Hecht and H. Hasumi, Eds., American Geophysical Union, Vol. 177, 319-338.

Frankignoul, C. and P. Müller, 1979: Quasi-geostrophic response of an infinite $\beta$ plane ocean to stochastic forcing by the atmosphere. J. Phys. Oceanogr., 9, 104-127.

Fu, L.-L., 2009: Pattern and velocity of propagation of the global ocean eddy variability. J. Geophys. Res., 114, C11 017.

Fu, L.-L., D. B. Chelton, P.-Y. L. Traon, and R. Morrow, 2010: Eddy dynamics from satellite altimetry: progress and challenges. Oceanography, 23, 14-25.

Fu, L.-L. and G. R. Flierl, 1980: Non-linear energy and enstrophy transfers in a realistically stratified ocean. Dyn. Atmos. Oceans, 4, 219-246.

Galperin, B., H. Nakano, H.-P. Huang, and S. Sukoriansky, 2004: The ubiquitous zonal jets in the atmospheres of giant planets and Earth's oceans. Geophys. Res. Lett., 31, L13 303.

Gebbie, G., 2007: Does eddy subduction matter in the northeast Atlantic Ocean? J. Geophys. Res., 112, C06007.

Gent, P. R. and J. C. Mcwilliams, 1990: Isopycnal mixing in ocean circulation models. J. Phys. Oceanogr., 20, 150-155.

Gill, A. E., J. S. A. Green, and A. J. Simmons, 1974: Energy partition in the largescale ocean circulation and the production of mid-ocean eddies. Deep-Sea Res., 21, 499-528.

Gille, S. T., K. Speer, J. R. Ledwell, and A. C. N. Garabato, 2007: Mixing and stirring in the southern ocean. EOS Trans. Amer. Geophys. Union, 88, 382-383. 
Greatbatch, R. J., X. Zhai, J. D. Kohlmann, and L. Czeschel, 2010: Ocean eddy momentum fluxes at the latitudes of the Gulf Stream and the Kuroshio extensions as revealed by satellite data. Ocean Dyn., 60, 617-628.

Gregory, J. M., 2000: Vertical heat transports in the ocean and their effect on timedependent climate change. Clim. Dyn., 16, 501-515.

Griesel, A., S. T. Gille, J. Sprintall, J. L. McClean, and M. E. Maltrud, 2009: Assessing eddy heat flux and its parameterization: A wavenumber perspective from a $1 / 10^{\circ}$ ocean simulation. Ocean Modelling, 29, 248-260.

Hall, M. M., 1991: Energetics of the Kuroshio Extension at $35^{\circ} \mathrm{N}, 152^{\circ}$ E. J. Phys. Oceanogr., 21, 958-975.

Held, I. M. and V. D. Larichev, 1996: A scaling theory for horizontally homogeneous, baroclinically unstable flow on a beta-plane. J. Atmos. Sci., 53, 946-952.

Held, I. M., M. Winton, K. Takahashi, T. Delworth, F. Zeng, and G. K. Vallis, 2010: Probing the fast and slow components of global warming by returning abruptly to preindustrial forcing. J. Climate, 23, 2418-2427.

Helfrich, K. R. and J. Pedlosky, 1993: Time-dependent isolated anomalies in zonal flows. J. Fluid Mech., 251, 377-409.

Helfrich, K. R. and J. Pedlosky, 1995: Large-amplitude coherent anomalies in baroclinic zonal flows. J. Atmos. Sci., 52, 1615-1629.

Hong, X., S. W. Chang, S. Raman, L. K. Shay, and R. Hodur, 2000: The interaction between hurricane Opal (1995) and a warm core ring in the Gulf of Mexico. Mon. Wea. Rev., 128, 1347-1365.

Hristova, H. G., J. Pedlosky, and M. A. Spall, 2008: Radiating instability of a meridional boundary current. J. Phys. Oceanogr., 38, 2294-2307.

Huang, B., P. H. Stone, A. P. Sokolov, and I. V. Kamenkovich, 2003: Ocean heat uptake in transient climate change: mechanisms and uncertainty due to subgridscale eddy mixing. J. Climate, 16, 3344-3356.

Huang, R. X., 1998: Mixing and available potential energy in a Boussinesq ocean. J. Phys. Oceanogr., 28, 669-678.

Huang, R. X., 2004: Energy flows in the ocean. Encyclopedia of Energy, Elsevier Inc., 497-509.

Huang, R. X., 2005: Available potential energy in the world's oceans. J. Mar. Res., 63, 141-158. 
Huang, R. X., 2010: Ocean circulation, wind driven and themohaline processes. Cambridge University Press, 806pp.

Hughes, G. O., A. M. Hogg, and R. W. Griffiths, 2009: Available potential energy and irreversible mixing in the meridional overturning circulation. J. Phys. Oceanogr., 39, 3130-3146.

Ivchenko, V. O., S. Danilov, and D. Olbers, 2008: Eddies in numerical models of the Southern Ocean. Ocean modeling in an eddying regime, Geophys. Monogr. Ser., M. W. Hecht and H. Hasumi, Eds., American Geophysical Union, Vol. 177, 177198.

Jackett, D. R. and T. J. McDougall, 1995: Minimal adjustment of hydrographic profiles to achieve static stability. J. Atmos. Oceanic Technol., 12, 381-389.

Jacob, J. P., E. P. Chassignet, and W. K. Dewar, 2002: Influence of topography on the propagation of isolated eddies. J. Phys. Oceanogr., 32, 2848-2869.

Jayne, S. R. and J. Marotzke, 2002: The oceanic eddy heat transport. J. Phys. Oceanogr., 32, 3328-3345.

Jia, Y., P. H. R. Calil, E. P. Chassignet, E. J. Metzger, J. T. Potemra, K. J. Richards, and A. J. Wallcraft, 2011: Generation of mesoscale eddies in the lee of the Hawaiian Islands. J. Geophys. Res., 116, C11009.

Johnson, T. J., R. H. Stewart, C. K. Shum, and B. D. Tapley, 1992: Distribution of Reynolds stress carried by mesoscale variability in the Antarctic Circumpolar Current. Geophys. Res. Lett., 19, 1201-1204.

Kamenkovich, I., P. Berloff, and J. Pedlosky, 2009a: Anisotropic material transport by eddies and eddy-driven currents in a model of the North Atlantic. J. Phys. Oceanogr., 39, 3162-3175.

Kamenkovich, I., P. Berloff, and J. Pedlosky, 2009b: Role of eddy forcing in the dynamics of multiple zonal jets in a model of the North Atlantic. J. Phys. Oceanogr., 39, 1361-1379.

Kaspi, Y. and G. R. Flierl, 2007: Formation of jets by baroclinic instability on gas planet atmospheres. J. Atmos. Sci., 64, 3177-3194.

Kessler, W. S. and L. Gourdeau, 2006: Wind-driven zonal jets in the South Pacific Ocean. Geophys. Res. Lett., 33, L03 608.

Killworth, P. D., D. B. Chelton, and R. A. de Szoeke, 1997: The speed of observed and theoretical long extratropical planetary waves. J. Phys. Oceanogr., 27, 1946-1966. 
Klocher, A., R. Ferrari, and J. H. LaCasce, 2012a: Estimating suppression of eddy mixing by mean flows. J. Phys. Oceanogr., 42, 1566-1576.

Klocher, A., R. Ferrari, J. H. LaCasce, and S. Merrifield, 2012b: Reconciling floatbased and tracer-based estimates of eddy diffusivities. J. Mar. Res., in press.

Kundu, P. K. and I. M. Cohen, 2004: Fluid mechanics. Elsevier Academic Press, 759pp.

Kuo, A., R. A. Plumb, and J. Marshall, 2005: Transformed Eulerian-mean theory. Part II: Potential vorticity homogenization, and the equilibrium of a wind- and buoyancy-driven zonal flow. J. Phys. Oceanogr., 35, 175-187.

Lachkar, Z., J. C. Orr, J. Dutay, and P. Delecluse, 2009: On the role of mesoscale eddies in the ventilation of Antarctic intermediate water. Deep-Sea Res. I, 56, 909-925.

Large, W., J. McWilliams, and S. Doney, 1994: Oceanic vertical mixing: a review and a model with nonlocal boundary layer parameterization. Rev. Geophys., 32, 363-403.

Lenn, Y. D., T. K. Chereskin, J. Sprintall, and J. L. McClean, 2011: Near-surface eddy heat and momentum fluxes in the Antarctic Circumpolar Current in Drake Passage. J. Phys. Oceanogr., 41, 1385-1407.

Liang, X. S. and A. R. Robinson, 2007: Localized multi-scale energy and vorticity analysis: II. Finite-amplitude instability theory and validation. Dyn. Atmos. Oceans, 44, 51-76.

Lorenz, E. N., 1955: Available potential energy and the maintenance of the general circulation. Tellus, 7, 157-167.

Maltrud, M. and G. K. Vallis, 1991: Energy spectra and coherent structures in forced two-dimensional and geostrophic turbulence. J. Fluid Mech., 228, 321-342.

Margules, M., 1905: On the energy of storms. Transl. C. Abbe in Smithson. Misc. Collect., 51, 533-595.

Marshall, J., A. Adcroft, C. Hill, L. Perelman, and C. Heisey, 1997a: A finite-volume, incompressible Navier Stokes model for studies of the ocean on parallel computers. J. Geophys. Res., 102, 5753-5766.

Marshall, J., C. Hill, L. Perelman, and A. Adcroft, 1997b: Hydrostatic, quasihydrostatic, and nonhydrostatic ocean modeling. J. Geophys. Res., 102, 5733-5752. 
Marshall, J. and T. Radko, 2003: Residual-mean solutions for the Antarctic Circumpolar Current and its associated overturning circulation. J. Phys. Oceanogr., 33, 2341-2354.

Marshall, J. and F. Schott, 1999: Open-ocean convection: observations, theory, and models. Rev. Geophys., 37, 1-64.

Marshall, J. C., 1984: Eddy-mean-flow interaction in a barotropic ocean model. Quart. J. Roy. Met. Soc., 110, 573-590.

Marshall, J. C. and G. J. Shutts, 1981: A note on rotational and divergent eddy fluxes. J. Phys. Oceanogr., 11, 1677-1680.

Maximenko, N. A., B. Bang, and H. Sasaki, 2005: Observational evidence of alternating zonal jets in the world ocean. Geophys. Res. Lett., 32, L12 607.

Maximenko, N. A., O. V. Melnichenko, P. P. Niiler, and H. Sasaki, 2008: Stationary mesoscale jet-like features in the ocean. Geophys. Res. Lett., 35, L08 603.

Maximenko, N. A. and P. P. Niiler, 2005: Hybrid decade-mean global sea level with mesoscale resolution. Recent Advances in Marine Science and Technology, N. Saxena, Ed., Honolulu: PACON International., 55-59.

Mazloff, M., P. Heimbach, and C. Wunsch, 2010: An eddy-permitting Southern Ocean state estimate. J. Phys. Oceanogr., 40, 880-899.

McClean, J. L., S. Jayne, M. E. Maltrud, and D. P. Ivanova, 2008: The fidelity of ocean models with explicit eddies. Ocean modeling in an eddying regime, Geophys. Monogr. Ser., M. W. Hecht and H. Hasumi, Eds., American Geophysical Union, Vol. 170, 149-163.

McWilliams, J. C. and G. R. Flierl, 1979: On the evolution of isolated, nonlinear vortices. J. Phys. Oceanogr., 9, 1155-1182.

McWilliams, J. C., W. R. Holland, and J. S. Chow, 1978: A description of numerical Antarctic Circumpolar Currents. Dyn. Atmos. Oceans, 2, 213-291.

Menemenlis, D., J. Campin, P. Heimbach, C. Hill, T. Lee, A. Nguyen, M. Schodlock, and H. Zhang, 2008: ECCO2: High resolution global ocean and sea ice data synthesis. Mercator Ocean Quarterly Newsletter, 31, 13-21.

Menemenlis, D., I. Fukumori, and T. Lee, 2005a: Using Green's functions to calibrate an ocean general circulation model. Mon. Wea. Rev., 133, 1224-1240.

Menemenlis, D., et al., 2005b: NASA supercomputer improves prospects for ocean climate research. Eos Trans. AGU, 86, 89, 95-96. 
Mohebalhojeh, A. R. and J. Theiss, 2011: The assessment of the equatorial counterpart of the quasi-geostrophic model. Quart. J. Roy. Met. Soc., 137, 1327-1339.

Morrow, R., J. Church, R. Coleman, D. Chelton, and N. White, 1992: Eddy momentum flux and its contribution to the Southern Ocean momentum balance. Nature, 357, 482-484.

Müller, P. and C. Frankignoul, 1981: Direct atmospheric forcing of geostrophic eddies. J. Phys. Oceanogr., 11, 287-308.

Nakano, H. and H. Hasumi, 2005: A series of zonal jets embedded in the broad zonal flows in the Pacific obtained in eddy-permitting ocean general circulation models. J. Phys. Oceanogr., 35, 474-488.

Nikurashin, M. and R. Ferrari, 2010: Radiation and dissipation of internal waves generated by geostrophic motions impinging on small-scale topography: Theory. $J$. Phys. Oceanogr., 40, 1055-1074.

Nishida, H. and W. White, 1982: Horizontal eddy fluxes of momentum and kinetic energy in the near-surface of the Kuroshio Extension. J. Phys. Oceanogr., 35, 165174.

Nishikawa, S., H. Tsujino, K. Sakamoto, and H. Nakano, 2010: Effects of mesoscale eddies on subduction and distribution of subtropical mode water in an eddyresolving OGCM of the western North Pacic. J. Phys. Oceanogr., 40, 1748-1765.

Okuno, A. and A. Masuda, 2003: Effect of horizontal divergence on the geostrophic turbulence on a beta-plane: suppression of the Rhines effect. Physics of Fluids, 15, 56-65.

Oort, A., L. Anderson, and J. Peixóto, 1994: Estimates of the energy cycle of the oceans. J. Geophys. Res., 99, 7665-7688.

Oort, A., S. Ascher, S. Levitus, and J. Peixóto, 1989: New estimates of the available potential energy in the world ocean. J. Geophys. Res., 94, 3187-3200.

O'Reilly, C. H., A. Czaja, and J. H. LaCasce, 2012: The emergence of zonal ocean jets under large-scale stochastic wind forcing. Geophys. Res. Lett., 39, L11 606.

Panetta, R. L., 1993: Zonal jets in wide baroclinically unstable regions: persistence and scale selection. J. Atmos. Sci., 50, 2073-2106.

Paulson, C. A. and J. J. Simpson, 1977: Irradiance measurements in the upper ocean. J. Phys. Oceanogr., 7, 952-956.

Pedlosky, J., 1984: The equations for geostrophic motion in the ocean. J. Phys. Oceanogr., 448, 455. 
Pedlosky, J., 1987: Geophysical Fluid Dynamics. Springer-Verlag, NY, 710pp.

Penny, A. B., A. P. Showman, and D. S. Choi, 2010: Suppression of the Rhines effect and the location of vortices on Saturn. J. Geophys. Res., 115, E02 001.

Piecuch, C. G. and R. M. Ponte, 2011: Mechanisms of interannual steric sea level variability. Geophys. Res. Lett., 38, L15 605.

Plumb, A. R. and R. Ferrari, 2005: Transformed Eulerian-mean theory. Part I: nonquasigeostrophic theory for eddies on a zonal-mean flow. J. Phys. Oceanogr., 35, $165-174$.

Polvani, L. M., J. C. McWilliams, M. A. Spall, and R. Ford, 1994: The coherent structures of shallow-water turbulence: Deformation-radius effects, cyclone/anticyclone asymmetry and gravity-wave generation. Chaos, 4(2), 177-186.

Poulin, F. J., G. R. Flierl, and J. Pedlosky, 2010: The baroclinic adjustment of time-dependent shear flows. J. Phys. Oceanogr., 40, 1851-1865.

Radko, T. and M. E. Stern, 1999: On the propagation of oceanic mesoscale vortices. J. Fluid Mech., 380, 39-57.

Rhines, P. B., 1975: Waves and turbulence on a beta-plane. J. Fluid Mech., 69, 417-443.

Rhines, P. B., 1979: Geostrophic turbulence. Ann. Rev. of Fluid Mech., 11, 404-441.

Richards, K. J., N. A. Maximenko, F. O. Bryan, and H. Sasaki, 2006: Zonal jets in the Pacific Ocean. Geophys. Res. Lett., 33, L03605.

Risien, C. M. and D. B. Chelton, 2008: A global climatology of surface wind and wind stress fields from eight years of QuikSCAT scatterometer data. J. Phys. Oceanogr., 38, 2379-2413.

Robinson, A. R. and J. C. McWilliams, 1974: The baroclinic instability of the open ocean. J. Phys. Oceanogr., 4, 281-294.

Roquet, F., C. Wunsch, and G. Madec, 2011: On the patterns of wind-power input to the ocean circulation. J. Phys. Oceanogr., 41, 2328-2342.

Rypina, I. I., I. Kamenkovich, P. Berloff, and L. J. Pratt, 2012: Eddy-induced particle dispersion in the near-surface North Atlantic. J. Phys. Oceanogr., in press.

Salmon, R., 1978: Two-layer quasigeostrophic turbulence in a simple special case. Geophys. Astrophys. Fluid Dyn., 10, 25-52. 
Sayanagi, K. M., A. P. Showman, and T. E. Dowling, 2008: The emergence of multiple robust zonal jets from freely evolving, three-dimensional stratified geostrophic turbulence with applications to Jupiter. J. Atmos. Sci., 65, 3947-3962.

Schlax, M. G. and D. B. Chelton, 2008: The influence of mesoscale eddies on the detection of quasi-zonal jets in the ocean. Geophys. Res. Lett., 35, L24602.

Schlax, M. G., D. B. Chelton, and M. H. Freilich, 2001: Sampling errors in wind fields constructed from single and tandem Scatterometer datasets. J. Atmos. Oceanic Technol., 18, 1014-1036.

Scott, R. B. and F. Wang, 2005: Direct evidence of an oceanic inverse kinetic energy cascade from satellite altimetry. J. Phys. Oceanogr., 35, 1650-1666.

Scott, R. B. and Y. Xu, 2009: An update on the wind power input to the surface geostrophic flow of the world ocean. Deep-Sea Res. I, 56, 295-304.

Sen, A., R. B. Scott, and B. K. Arbic, 2008: Global energy dissipation rate of deepocean low-frequency flows by quadratic bottom boundary layer drag: computations from current-meter data. Geophys. Res. Lett., 35, L09 606.

Shay, L. K., G. J. Goni, and P. G. Black, 2000: Effects of a warm oceanic feature on hurricane Opal. Mon. Wea. Rev., 128, 1366-1383.

Shuckburgh, E. H. J., J. Marshall, and C. Hill, 2009: Robustness of an effective diffusivity diagnostic in oceanic flows. J. Phys. Oceanogr., 39, 1993-2009.

Smith, K. S., 2007: The geography of linear baroclinic instability in Earth's oceans. J. Mar. Res., 65, 655-683.

Smith, W. and D. Sandwell, 1997: Global sea floor topography from satellite altimetry and ship depth soundings. Science, 277, 1956-1962.

Spall, M. A., 2000: Generation of strong mesoscale eddies by weak ocean gyres. J. Mar. Res., 58, 97-116.

Spall, M. A., 2012: Influences of precipitation on water mass transformation and deep convection. J. Phys. Oceanogr., 42, 1684-1700.

Srinivasan, K. and W. R. Young, 2012: Zonostrophic instability. J. Atmos. Sci., 69, $1633-1656$.

Stommel, H., A. B. Arons, and A. J. Faller, 1958: Some examples of stationary planetary flow patterns in bounded basins. Tellus, 10, 179-187. 
Taguchi, B., R. Furue, N. Komori, A. Kuwano-Yoshida, M. Nonaka, H. Sasaki, and W. Ohfuchi, 2012: Deep oceanic zonal jets constrained by fine-scale wind stress curls in the South Pacific Ocean: A high-resolution coupled GCM study. Geophys. Res. Lett., 39, L08602.

Tanaka, Y. and K. Akitomo, 2010: Alternating zonal flows in a two-layer wind-driven ocean. J. Oceangr., 66, 475-487.

Theiss, J., 2004: Equatorward energy cascade, critical latitude, and the predominance of cyclonic vortices in geostrophic turbulence. J. Phys. Oceanogr., 34, 1663-1678.

Theiss, J., 2006: A generalized Rhines effect and storms on Jupiter. Geophys. Res. Lett., 33, L08 809.

Theiss, J. and A. Mohebalhojeh, 2009: The equatorial counterpart of the quasigeostrophic model. J. Fluid Mech., 637, 327-356.

Thompson, A. F., 2008: The atmospheric ocean: eddies and jets in the Antarctic Circumpolar Current. Philos. Trans. R. Soc. London, 366, 4529-4541.

Thompson, A. F., 2010: Jet formation and evolution in baroclinic turbulence with simple topography. J. Phys. Oceanogr., 40, 257-278.

Thompson, A. F. and K. J. Richards, 2011: Low frequency variability of Southern Ocean jets. J. Geophys. Res., 116, C09022.

Tulloch, R., J. Marshall, C. Hill, and K. S. Smith, 2011: Scales, growth rates, and spectral fluxes of baroclinic instability in the ocean. J. Phys. Oceanogr., 41, 10571076.

Tulloch, R., J. Marshall, and K. S. Smith, 2009: Interpretation of the propagation of surface altimetric observations in terms of planetary waves and geostrophic turbulence. J. Geophys. Res., 114, C02 005.

Vallis, G. K., 2006: Atmospheric and oceanic fluid dynamics. Cambridge University Press, 745pp.

van Sebille, E., I. Kamenkovich, and J. K. Willis, 2011: Quasi-zonal jets in 3-D Argo data of the northeast Atlantic. Geophys. Res. Lett., 38, L02 606.

Venaille, A., G. K. Vallis, and K. S. Smith, 2011: Baroclinic turbulence in the ocean: analysis with primitive equation and quasigeostrophic simulations. J. Phys. Oceanogr., 41, 1605-1623.

Voet, G. and D. Quadfasel, 2010: Entrainment in the Denmark Strait overflow plume by mesoscale eddies. Ocean Sci., 6, 301-310. 
Volkov, D., L.-L. Fu, and T. Lee, 2010: Mechanisms of the meridional heat transport in the Southern Ocean. Ocean Dyn., 60, 791-801.

Volkov, D. L. and L.-L. Fu, 2008: The role of vorticity fluxes in the dynamics of the Zapiola Anticyclone. J. Geophys. Res., 113, C11015.

Volkov, D. L., T. Lee, and L.-L. Fu, 2008: Eddy-induced meridional heat transport in the ocean. Geophys. Res. Lett., 35, L20 601.

von Storch, J., C. Eden, I. Fast, H. Haak, D. Hernndez-Deckers, E. Maier-Reimer, J. Marotzke, and D. Stammer, 2012: An estimate of Lorenz energy cycle for the world ocean based on the $1 / 10^{\circ}$ STORM/NCEP simulation. J. Phys. Oceanogr., in press.

Wang, J., 2011: Instabilities of an eastern boundary current with and without largescale flow influence. Ph.D. thesis, MIT/WHOI, 227pp.

Wang, J., M. A. Spall, G. R. Flierl, and P. Malanotte-Rizzoli, 2012: A new mechanism for the generation of quasi-zonal jets in the ocean. Geophys. Res. Lett., 39, L10 601.

Waterman, S. N. and S. R. Jayne, 2011: Eddy-mean flow interactions in the alongstream development of a western boundary current jet: an idealized model study. J. Phys. Oceanogr., 41, 682-707.

Whitehead, J., 1975: Mean flow driven by circulation on a beta plane. Tellus, 27, 358-364.

Wilkin, J. L. and R. A. Morrow, 1994: Eddy kinetic energy and momentum flux in the Southern Ocean: Comparison of a global eddy-resolving model with altimeter, drifter, and current-meter data. J. Geophys. Res., 99, 7903-7916.

Williams, R. G., C. Wilson, and C. W. Hughes, 2007: Ocean and atmosphere storm tracks: The role of eddy vorticity forcing. J. Phys. Oceanogr., 37, 2267-2289.

Winters, K. B., P. N. Lombard, J. J. Riley, and E. A. D'Asaro, 1995: Available potential energy and mixing in density-stratified fluids. J. Fluid Mech., 289, 115128.

Witter, D. L. and D. B. Chelton, 1998: Eddy-mean flow interaction in zonal oceanic jet flow along zonal ridge topography. J. Phys. Oceanogr., 28, 2019-2039.

Wortham, C. J. L., 2012: A multi-dimensional spectral description of ocean variability with applications. Ph.D. thesis, MIT/WHOI, 184pp.

Wu, C., C. Lee, and I.-I. Lin, 2007: The effect of the ocean eddy on tropical cyclone intensity. J. Atmos. Sci., 64, 3562-3578. 
Wunsch, C., 1997: The vertical partition of oceanic horizontal kinetic energy. J. Phys. Oceanogr., 27, 1770-1794.

Wunsch, C., 1998: The work done by the wind on the oceanic general circulation. J. Phys. Oceanogr., 28, 2332-2340.

Wunsch, C., 2010: Towards a mid-latitude ocean frequency-wavenumber spectral density and trend determination. J. Phys. Oceanogr., 40, 2264-2281.

Wunsch, C., 2011: The decadal mean ocean circulation and Sverdrup balance. J. Mar. Res., 69, 417-434.

Wunsch, C. and R. Ferrari, 2004: Vertical mixing, energy, and the general circulation of the oceans. Ann. Rev. of Fluid Mech., 36, 281-314.

Wunsch, C. and P. Heimbach, 2007: Practical global oceanic state estimation. Physica D., 230, 197-208.

Wunsch, C., P. Heimbach, R. Ponte, I. Fukumori, and the ECCO-Consortium members, 2009: The global general circulation of the oceans estimated by the ECCOConsortium. Oceanography, 22, 89-103.

Wunsch, C. and D. Stammer, 1998: Satellite altimetry, the marine geoid and the oceanic general circulation. Annu. Rev. Earth Planet. Sci., 26, 219-253.

Xu, C., X. Shang, and R. X. Huang, 2011: Estimate of global eddy energy generation/dissipation rate from altimetry data. Ocean Dyn., 61, 525-541.

Zhai, X., H. L. Johnson, D. P. Marshall, and C. Wunsch, 2012: On the wind power input to the ocean general circulation. J. Phys. Oceanogr., 42, 1357-1365.

Zhai, X. and D. P. Marshall, 2012: Vertical energy fluxes in the North Atlantic and subpolar gyres. J. Phys. Oceanogr., accepted. 